


\section{THE LIBRARY BRIGHAM YOUNG UNIVERSITY PROVO, UTAH}


Digitized by the Internet Archive in 2011 with funding from Brigham Young University

http://www.archive.org/details/lesremdesdesch00saff 



LES

REMÈDES DES CHAMPS

PREMIẺHE PARTIE 
COULOMHIERS. - TYP. PAUL BRODARD ET Cle.

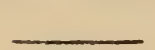




\title{
DOCTEUR SAFFRAY
}

\subsection{3 \\ la 19 LES \\ EMEDES DES CHAVIPS \\ HERBORISATIONS PRATIQUES}

A L'USAGE DES INSTITUTEURS, DES ECCLÉsiastiques

T DE TOUS CEUX QUI DONNENT LEURS SOINS AUX MALADES

Ouvrage couronné

par la Société libre d'instruction et d'éducation populaires

\author{
PREMIÉRE PARTIE \\ OCTOBRE A MARS \\ contenant 75 figures
}

CINQUIÈME ÉDITION

\section{PARIS}

LIBRAIRIE HACHETTE ET Cie

79, Bouleyard SAINT-Germain, 79 


\section{THE LIBRARY \\ BRIGHAM YOUNG UNIVERSITY PROVO, UTAH}




\section{INTRODUCTION}

Le jour viendra sans doute où l'on organiэra, dans les campagnes, un service médical ssez complet pour que la chaumière la plus auvre et la plus isolée puisse recevoir la visite u médecin toutes les fois qu'elle sera nécesaire. On verra en même temps disparaître la révention assez commune chez le paysan con:e la médecine officielle. Mais en prenant les hoses telles qu'elles sont aujourd'hui, il est faile de constater, d'une part, que le nombre des rédecins est insuffisant, et que, de l'autre, l'haitant des campagnes donne volontiers la préféznce aux remèdes indiqués par un voisin ou ar un guérisseur de village. A la campagne, n se méfie des drogues de pharmacien, peuttre un peu parce qu'elles coûtent cher, l'on 
confiance dans les simples. De plus, la méde cine des pauvres y reste souvent entre les main de personnes charitables qui ne savent et $n$ peuvent employer que les ressources des champ où s'exercent leur officieux ministère.

Leur bonne volonté, leur dévouement ont be soin d'être guidés, éclairés, et nous leur dédion ce petit livre, dans lequel nous adoptons $l_{\text {: }}$ forme familière d'entretiens d'un instituteu âvec ses élèves les plus avancés. 


\section{LES}

\section{REMÈDES DES CHAMPS}

\section{HERBORISATIONS PRATIQUES}

Nous nous proposons, dans ces herborisations ratiques, de diriger les instituteurs, les ecclésiastiues, les volontaires de l'art de guérir, dans la foriation de l'herbier des plantes médicinales qui oissent spontanément dans leur canton ou qui ouvent y être cultivées. Nous indiquerons pour taque plante son histoire, ses propriétés, son emoi. Nous combattrons les préjugés, les erreurs spulaires qui attribuent à certains végétaux des zrtus imaginaires ou conduisent à en faire un im-udent usage ; car s'il est dangereux d'administrer ns connaissances suffisantes une substance énerque, on peut arriver à un résultat non moins fal par l'emploi d'une substance inerte, alors que la e du malade dépend de la promptitude des secours. Dans les indications que nous donnerons au sujet - la médecine domestique, nous nous efforcerons ne d'établir la propriété la plus marquante de 
chaque plante, sa manière d'agir sur l'homme e sur les animaux, autant qu'on peut la connaitre la limite de son emploi en l'absence du médecin et nous laisserons de côté tout ce qui est sujet controverse ou réclame de nouvelles expériences Nous ne craindrons pas de sortir souvent de notr cadre spécial pour étudier, au point de vue écono mique et industriel, les plantes dont nous nous oc cuperons, et nous saisirons toutes les occasion d'entrer à leur sujet dans des explications botani ques dont l'ensemble embrassera les notions élé mentaires indispensables à la formation du plu simple herbier.

Nous ne nous pornerons pas à indiquer le moyens de reconnaître, de cueillir et de conserve les plantes; nous donnerons aussi quelques ins tructions sur leur culture, afin que l'instituteu puisse leur consacrer une partie de son jardin. Tou tefois, on n'oubliera pas que les plantes sauvage: sont d'ordinaire plus énergiques que celles sou mises à la culture. Ce petit jardin botanique, $s$ modeste qu'il soit, offrirait au maitre une ressourc importante pour ses leçons alors qu'une excursior dans la campagne serait impossible, et lui permet trait de suivre jour par jour avec ses élèves le phénomènes les plus curieux de la végétation.

Mais c'est en pleine campagne, dans les prés, le jachères, les bois, le long des fossés, au bord de: ruisseaux, près des marécages et sur les monta gnes, que se donneront les leçons les plus at. trayantes et les plus fructueuses. Là, dans un Intimité qui rend la parole persuasive et la gravi sans effort dans la mémoire et dans le cœur, I maitre causera de science, encouragera les ques. 
tions, lancera à la piste d'échantillons, et, choisissant un type convenable, trouvera moyen d'intéresser son curieux auditoire, avant d'avoir glissé aucun mot technique ou risqué un nom latin. Il se gardera bien de commencer par expliquer pendant plusieurs heures les mots Pétale, Pistil, Ovaire, Monocotylédone, Phanérogame, etc. : ce serait effaroucher ses jeunes amis, ce serait faire de la science, et ils sont conviés à une promenade, à une partie de plaisir. Mais une fois l'intérêt éveillé, ils retiendront sans peine, à chaque séance, quelques termes étrangers, quelques mots rébarbatifs, et, au bout de quelque temps, ils auront acquis les éléments de la théorie en même temps que la pratique, et seront botanistes, sans le savoir. Que de choses on pourrait apprendre ainsi !

Comme il importe de cueillir les plantes à l'époque oủ les parties actives sont dans toute leur vigueur, nous avons divisé nos promenades par saisons au point de vue de leur récolte. Cette division ne peut être rigoureuse, puisque la floraison de beaucoup d'espèces et la maturation de leurs graines ou de leurs fruits peut varier en France dans une limite de cinq à six semaines. Lorsqu'il s'agit d'une racine, d'une graine, d'une écorce, le moment de la récolte n'est pas toujours opportun pour étudier la plante qui la fournit; dans ce cas, nous aurons soin de la faire remarquer incidemment dans $\epsilon$ cours de nos causeries et nous n'aurons qu'à rappeler quelques détails quand nous en ferons l'étude complète. Voici quelle sera la manière la plus ordinaire de procéder dans nos excursions : choisiscant une plante comme type d'une certaine pro- 
prièté médicale, nous expliquerons son usage, son emploi, ses effets, son histoire, pendant qu'elle passera de main en main. Nous dirons alors à quelle famille elle appartient, ayant soin d'avoir tout prêt un échantillon type de cette famille, au moyen duquel nous indiquerons les principaux caractères qui la distinguent. Nous citerons alors les diverses plantes de cette famille qui nous sont familières, procédant toujours par associations de faits et d'images. Puis revenant à la propriété médicale, nous grouperons autour de la plante type celles qui jouissent des mêmes vertus à divers degrés.

Par ce moyen, nous espérons éviter l'aridité d'un cours de Botanique ou d'un traité de Matière médicale. L'esprit sera toujours tenu en éveil par la comparaison des objets, l'enchaînement des idées, le rapprochement des faits. De nombreuses répétitions, dans un ordre préconçu, favoriseront la mémoire. Les définitions, les termes techniques indispensables, appris peu à peu, sans effort, formeront, dans leur ensemble, les notions d'un cours élémentaire de Botanique; la curiosité sera toujours entretenue et satisfaite. Voilà sur quoi nous comptons pour faire aimer nos promenades et rendre fructueuse notre école buissonnière.

Nous chercherons toujours lo langage le plus simple et conserverons avec soin les noms vulgaires des plantes qui sont les plus familiers; le nom latin, mis en regard des noms vulgaires, dans l'herbier, mais que les commençants ne seront pas tenus de connaître, suffira pour éviter toute erreur.

Nous n'avons point la prétention d'offrir un traité complet, d'autant moins utile en pratique qu'il serait plus étendu. Nous présentons simplement un 
cadre que chacun pourra varier selon les circonstances, selon ses goûts, ses études.

En dehors de l'utilité spéciale de nos promenades, nous croyons qu'elles pourront contribuer à développer l'amour des champs chez ceux qui sont appelés à y vivre. Il est certain que la démoralisante émigration vers les villes cessera lorsque le paysan comprendra la nature qui l'environne, les richesses qu'elle offre spontanément ou donne en échange du travail ; quand l'instruction, l'éducation, sources de moralisation et de bien-être, l'auront mis à même d'entrer en pleine possession de son domaine.

Les résultats immédiats, pratiques, ne manquent pas non plus d'importance. On envoie souvent les enfants faire de l'herbe, et, s'ils ne sont pas prévenus, ils mêlent à leur récolte de fourrage des plantes malsaines ou de véritables poisons. Les pâturages sont quelquefois infestés d'herbes vénéneuses; l'instinct n'en éloigne pas toujours les animaux, et l'on oréviendrait des accidents si l'on prenait soin de les létruire. Les pâtres pourraient utiliser les loisirs orcés de leur genre de vie en cueillant, dans les neilleures conditions possibles, les plantes médiciaales de leur canton, pour les vendre à la ville la lus proche.

Là où les secours du médecin sont forcément tarlifs, où la médecine domestique est le plus souvent a seule à laquelle on ait recours, par suite de rouine, de préjugés ou de pénurie, il importe de vul;ariser la connaissance des plantes; il est à souiaiter que chaque maison ait sa petite provision de imples. On en usera avec prudence, en attendant e médecin; on les aura sous la main, pour les em. Jloyer selon ses prescriptions. 
Les médecins de notre génération, même ceu: des campagnes, négligent malheureusement l'étud de nos végétaux indigènes. Beaucoup seraient em barrassés s'il leur fallait dire le nom et les proprié tés de plantes vulgaires que leur apporte un paysan beaucoup mêrne ne reconnaîtraient pas dans la cam pagne celles qu'ils prescrivent chaque jour, e dont ils n'ont vu que des fragments dans les bo caux d'une pharmacie. La mode a fait adopter de: plantes de l'Inde ou de l'Amérique, dont le seu mérite est d'agir d'une façon plus énergique quı celles douées des mêmes vertus sous nos climats Alors même que nous avions autour de nous dei agents aussi énergiques, on a donné la préférence aux produits lointains; puis est venue la médecin chimique, par qui les végétaux ont été relégués at second rang, et méme presque entièrement né. gligés.

Nous ne pensons pas avec Fernel qu'il existe en tre les plantes et les hommes d'un même clima: une espèce de sympathie ou de congénialité qui le: rendent spécifiques, mais nous sommes convaincu que, de l'équateur au $60^{\circ}$ degré de latitude, la Pro vidence bienfaisante a placé dans chaque pays toutes les plantes nécessaires à la guérison de no maladies. Et ce̊pendant nous portons à l'étrangel des millions pour payer des produits dont nous fou lons chaque jour l'équivalent sous nos pas.

Il y a donc tout avantage à vulgariser l'étude de nos plantes médicinales : on évitera des maux e des accidents, on élargira le domaine de la méde. cine domestique; le médecin se rendra plus popu. laire, et partant fera plus de bien, si l'on n'a pas : craindre d'aller chez l'apothicaire; l'herboristeris 
iendra une branche plus importante de comrce, et demandera ses approvisionnements aux itants des campagnes.

uissent ces considérations servir d'encourage. nt à ceux à qui nous nous adressons ; puissenttrouver dans ces promenades tout l'intérêt que as y prenons nous-même et faire en sorte que rs jeunes amis regardent comme une fête d'aller boriser sous leur direction.

Ivant d'entrer en campagne, faisons quelques ommandations sur la manière d'herboriser et de mer un herbier.

Totre bagage sera des plus simples : une ou pluurs boîtes en fer-blanc ou mieux en zinc; quelss cahiers de papier brouillard, maintenus entre ix planches et serrés par une ficelle, pour rece$r$ les plantes ou les portions de plantes les plus icates; un bâton ferré d'une bonne houlette; un teau à plusieurs lames; un sac pour les tuberes, les écorces, etc., et, s'il se peut, une loupe, ur l'examen des parties les plus ténues des véaux.

ans bien des cas, nous ne pourrons pas recueille même jour toutes les parties d'une plante né. saires pour former un échantillon complet. $\mathrm{S}:$ is détachons une écorce pendant l'hiver, nous' ons obligés de revenir, pendant la belle saison. per un rameau en fleur, et plus tard cueillir un it. Nos herborisations étant faites au point $d \epsilon$ spécial des vertus médicinales des plantes ou quelqu'une de leurs parties, nous devrons ré- 
colter la partie dont nous avons besoin au temps sa plus grande activité, afin de nous familiar avec l'apparence, l'odeur, la saveur caractéristiqu Puis, pour former une page d'herbier, nous y jc drons la plante entière, ou un rameau, ou au $\mathrm{mc}$ une fleur et une feuille.

La moisson du jour terminée, il faudra, le $\mathrm{f}$ tôt possible, sécher les plantes. Après avoir nett et mondé les échantillons, on les plongera pend quelques minutes dans de l'eau tiède légèrem alunée, et on les fera ressuyer entre des feuilles papier non collé, qui aura été d'avance passé à l' d'alun et séché. Les plantes n'offrant plus de tra d'humidité à l'extérieur, on place chacune er deux feuilles de papier aluné, et l'on superpose échantillons, en ayant soin de mettre entre eux cahier de papier non collé. Dix ou douze échan lons seront alors fixés entre deux planches et $\mathrm{s}$ mis à une pression modérée, au moyen d'une pre ou de poids quelconques. On augmentera gradue ment la pression pendant deux ou trois jours, ap lesquels les plantes seront exposées à un cour d'air dans un endroit sec.

Pour former les pages de l'herbier, on pren du papier collé un peu fort, préalablement aluné passant dessus une éponge imbibée d'eau d'alun l'on y fixera la plante ou ses diverses parties moyen de bandelettes de papier retenues par épingles. On inscrira au basle nom latin dela plar celui de la famille à laquelle elle appartient; noms vulgaires; le lieu et la date de la récolte; principales vertus médicales. Chaque page libres recouverte d'une feuille de papier non collé et alu et l'on formera avec ces pages des cahiers peu vo 
ux portant sur la couverture l'indication des lles qui s'y trouvent, suivie de la liste des plantes prenant seulement le nom français et le nom laChaque page étant numérotée, ainsi que chaque er, un catalogue général portant le numéro du er et de la page permettra de trouver immédiaent l'échantillon cherché.

herbier le plus feuilleté est celui qui se cono le mieux, car il est moins sujet aux attaques insectes. On doit, dans tous les cas, saupoude camphre les enveloppes de l'herbier, le conor dans un endroit sec, et l'exposer souvent au d air. Celui d'une école se trouvera dans d'exntes conditions, puisqu'il servira, pendant les s de pluie, à répéter les leçons données en plein Même si vous le destinez à une Exposition scone craignez pas de le laisser toucher par des is un peu maladroites ou même susceptibles aisser quelques traces de leur pression. On aii à constater que vous n'avez pas fait une ste collection de parade; une tache par-ci, par-

e sera comme les trous de balles d'un drapeau. 


\section{PREMIERE SÉRIE DE PROMENADES.}

(OCTOBRE, NOVEMBRE, DÉGeMBRE.)

Vous me demandez, mes amis, si nous i bien loin chercher des plantes. Je ne le pense car nous trouverons partout des sujets d'ensei ment. Le chemin, le fossé, le buisson, abonder végétaux utiles. En voulez-vous un exemple: gardez à vos pieds, qu'est-ce que cela? - C'er la mauvaise herbe. - Oui, on l'appelle sou ainsi, et comme elle croît vite, comme elle a le dure, on dit : mauvaise herbe croît toujours. herbe, excellente, commo vous allez voir, c'ei Chiendent (Triticum repens), ainsi nommé $p$ que les chiens et les chats la mangent pou purger, et surtout pour se faire vomir.

Nous appelons le chiendent mauvaise herbe $p$ qu'il nuit à nos cultures et qu'il est très-difficil le détruire, à cause de ses longues racines ram. dans toutes les directions et dont lo moindre $i$ ceau reproduit la plante.

Goûtez ces racines noueuses et blanchâtres, je viens de découvrir, vous trouverez que les tendres, les plus jeunes, ont une saveur lég ment sucrée. C'est qu'en effet elles contiennen1 sucre et de l'amidon. Voilà pourquoi, si vous faites bouillir dans do l'eau, après les avoir un écrasées, vous obtiendrez une tisane adoucissa légèrement diurétique et rafraîchissante. C'es tisane commune des hôpitaux. Les médecin rangent parmi les médicaments émollients, c 
à-dire capables de ramollir, de relâcher les par avec lesquelles on les met en contact, de call l'irritation, de diminuer la douleur. En se mê avec le sang, ils le délayent, lo rendent moins e: tant, et par conséquent font cesser les inflam, tions. Tous les médicaments de cette classe ( tiennent de la gomme, ou de la fécule, ou du su ou de l'huile, quelquefois plusieurs de ces su tances; leur odeur est faible, leur saveur douceâ

Le temps le plus favorable pour récolter la rac de chiendent est le mois d'octobre.

On choisit les plus jeunes, on sépare avec s les tiges, puis on les bat pour enlever l'épider qui donnerait à la décoction un goût âcre, on fait sécher par petites bottes. Il ne faut pas c server le chiendent plus d'une année, car les v l'attaquent facilement et en rongent la fécule.

La composition de cette racine fait supposer $t_{\text {i }}$ d'abord qu'elle est nutritive. Les anciens Egypti lui reconnaissaient cette propriété ; ils la réd saient en farine qu'ils mélangeaient avac celle blé, dans les temps de disette. Les peuples du No ont quelquefois recours au même expédient, prin palement en Pologne. On peut aussi en retirer sucre, et par la fermentation de ce sucre, obte. de l'alcool; de sorte que l'on a quelquefois fabriq avec la racine de chiendent, une bonne bière ménage. Voici comment on procède. Vous prer soit 4 kilogrammes de chiendent haché, vous mettez dans un baquet et l'arrosez de temps temps avec de l'eau tiède, de manière à le mai tenir très-humide, mais sans le couvrir d'eau. . bout de peu de jours vous voyez germer de peti pousses blanches. Lorsqu'elles ont atteint envir 
entimètre, vous mettez les racines dans un baril 1 kilog. de baies de genièvre concassées, 2 kil. ucre brut et environ 60 grammes de levûre de . Vous versez dessus 3 litres d'eau très-chaude muez; le lendemain, vous ajoutez 8 litres d'eau de et le troisième jour 9 litres, ayant soin de er un fausset d'évent pour le dégagement des produits par la fermentation. Au bout de cinq ix jours, vous soutirez dans un baril propre, ux jours après vous pouvez boire cette bière o et agréable. Vous voyez, mes amis, que une mauvaise herbe qui sert à quelque chose. faut avouer cependant que c'est un fléau pour amp qu'elle envahit, et vous devez désirer sacomment faire pour la détruire, car vous avez adu dire souvent que c'était impossible. Quand e vous essaieriez de retourner la terre à la et d'arracher toutes les racines, il vous en pperait toujours quelques fragments qui bienepeupleraient un grand espace. Le Chiendent oin d'humidité, il meurt dans une terre bien ablie, pendant les mois les plus chauds. Si cultivez dans le champ infesté une plante demande une terre bien préparée et un ou buttages, le Chiendent ne pourra résister. donnerez à la terre au moins deux labours ux hersages, pour la bien ameublir; puis, en vous y sèmerez des pommes de terre en donun troisième labour ; en juin vous y repiquerez betteraves. Les façons que nécessiteront ences plantes achèveront de tuer les derniers ons de Chiendent.

us avons dit que le Chiendent est une herbe. Cette e appartient à la famille la plus nombreuse et 
la plus utile des végétaux, la famille des Gramin (du latin gramen, herbe). Ces plantes sont fac à reconnaitre. La tige simple, ronde, ordinairem creuse, est divisée par des nœuds un peu renf d'où partent des feuilles étroites et longues embrassent en partie la tige ou chaume. La nat ne leur a pas donné les fleurs brillantes dont l'é et le parfum sont souvent l'apanage de végét. peu utiles. Les Graminées, qui comprennent I de 3000 plantes, constituent, en quelque sorte démocratie du règne végétal : elles représenten peuple, dont la vie simple, mais féconde, proc la richesse et la prospérité des nations. Ce sont Graminées qui forment ce tapis de verdure don terre se couvre naturellement dans tous les clin tempérés. Nous leur devons l'herbe de nos p ries, nourriture ordinaire des troupeaux. Le fruits ou graines sont la base de l'alimentation l'homme : le Blé, le Seigle, l'Orge, l'Avoine, d les régions froides ou tempérées; le Mil, le M le Sorgho, le Riz, dans les climats plus chav fournissent amplement à nos besoins. Leur cult remonte à l'antiquité la plus reculée. Plusie graminées sauvages pourraient, au besoin, con buer à l'alimentation de l'homme: tel est le Paty aquatique (Poa fluitans), dont la tige flotte sur eaux stagnantes, et que l'on appelle quelque. herbe-à-la-manne. En Prusse, les paysans la cueillent pour s'en nourrir.

Les roseaux appartiennent aussi à la famille Graminées. Dans les pays chauds, les Bambc roseaux gigantesques, s'élancent à vingt-cinq tres de hauteur, et leur tige creuse, grosse com celle d'un chêne de trente ans, sert à constri 
maisons, à fabriquer des meubles, des ustenes de ménage. Chez nous, les usages de la paille us sont familiers. Le sucre est produit, dans les htrées intertropicales, par l'évaporation du suc in roseau, la Canne à sucre; on en retire aussi du rgho, et même du Maìs, dans les régions chaudes. routes les graminées sont utiles; quelques-unes, us le voyez, constituent la principale richesse du oureur, et chose remarquable, il n'y en a que is ou quatre qui soient nuisibles à l'homme ou $x$ animaux. Parmi celles-ci, vous connaissez raie annuelle ou enivrante (Lolium temulen$m$ ), dontil est quelquefois difficile de débarrasser blés. La farine de ses graines, mêlée au pain ns la proportion d'un quinzième, cause déjà, ez l'homme, des effets semblables à ceux de l'iesse. L'ivraie annuelle est aussi un poison pour chiens, les moutons et le cheval. Il est donc portant de ne pas la confondre avec l'ivraie race ou faux froment (Lolium perenne) qui est contraire un excellent fourrage et dont on fait $s$ prairies artificielles.

Vous voyez que le Chiendent, que vous appeliez it à l'heure une mauvaise herbe, est très-bien parenté. Il appartient à une bonne et nombreuse nille, sans laquelle l'homme mènerait une vie sérable. Ayez toujours chez vous une petite proion de ses racines émollientes, adoucissantes et peu diurétiques. La tisane que l'on en fait est le pour les rhumes, la fièvre, et toutes les malais inflammatoires.

Mes amis, asseyons-nous à l'ombre, et faisons iventaire de notre récolte. 
Nous avons parlé tantôt des remèdes émollient ou adoucissants. Voici deux plantes que j'ai eu soi d'arracher avec leurs racines, et qui appartiennen à cetto classe. Celle-ci est la Grande Coxsoun

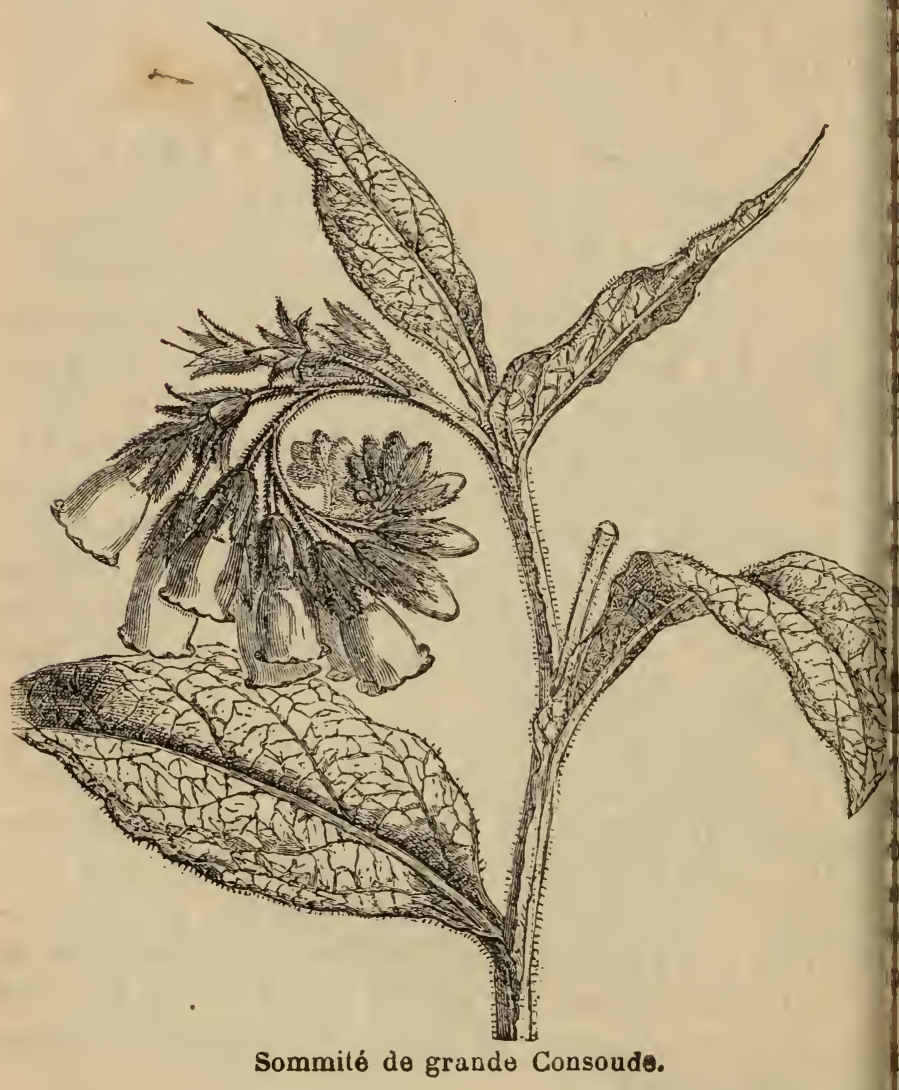

(Symphitum officinale), vulgairement appelé Oreille-d'âne, sans doute à cause de la forme de se feuilles, lorqu'elles ne sont pas complétemeut déve loppées. Quant à son nom de Consoude, il lui vien 
une erreur populaire répandue par les médecins. y a quelques siècles. Ils attribuaient à cette plante vertu de consolider, de réunir les organes, de uérir les fractures et d'arrêter les hémorrhagies en catrisant les vaisseaux ouverts des plaies. La Conude aime les terres grasses et humides, les prés, $\mathrm{s}$ fossés, les bois, le bord des ruisseaux et des ares. La plante est vivace, haute de 50 à 60 cenmetres. La tige est un peu branchue, velue, charue, anguleuse : les feuilles sont grandes, sans coupures, mais seulement un peu ondulées sur $\mathrm{s}$ bords, de forme ovale aiguë; elles sont alternes, est-à-dire qu'elles ne partent pas deux à deux du ême point de la tige, mais se trouvent, en face une de l'autre, séparées par un certain intervalle. Les fleurs, tubulées et un peu en cloche, se monent en mai et juin. Elles sont disposées en épis courbès et pendants; il y en a de rouges, de jauis et de blanches.

Les racines sont épaisses, à peine rameuses, bruis à l'extérieur, blanches en dedans, succulentes, uceâtres au goût, inodores, pleines d'un mucilage squeux auquel elles doivent leur propriété adoussante. Il est bon de les récolter au mois d'octo- o ou de novembre. Après les avoir bien nettoyées les coupe par tranches sur la longueur et on les che. Les surfaces mises à nu deviennent jaunes, is brunes.

La tisane de consoude se prépare en faisant bouil - dans un litre d'eau 60 grammes de racine donf a eu soin d'enlever l'écorce. Pour toutes les pré. rations que je vous indiquerai, on doit employer s vases de terre. La racine fraîche écrasée et apiquée sur les tumeurs enflammées, calme la dou- 
leur. Un cataplasme bien chaud fait avec cette ra. cine bouillie procure un soulagement notable dan: les accès de goutte, non point par une vertu spéci fique contre cette maladie, mais seulement par l. propriété adoucissante, analogue à celle de.la Gui mauve, de la graine de Lin, et de beaucoup d'autre plantes dont nous nous occuperons.

Il y a deux autres espèces de Consoude que l'os peut utiliser dans la petite culture, ce sont : l Consoude a Feuilles .Rudes et la Consoude héris sÉE. On les sème de bonne heure dans une terr douce et profonde, et dès le mois d'avril ellès four nissent un fourrage auquel les vaches s'accoutu ment aisément.

La Consoude appartient à la même famille que li bourrache, celle des Borraginées, qui tire son non de cette dernière plante, dont nous parlerons un autre fois.

Cette plante à feuilles oblongues, lisses, d'un ver clair tacheté de brun noirâtre, et dont la racin forme deux tubercules de la grosseur d'une noi. sette, c'est l'OrGhis taché (Orchis maculata), don vous avez vu au mois de mai les jolies fleurs pour prées disposées en longs épis, et que vous nommei Pentecôtes. Examinées avec attention, ces fleur: offrent une disposition particulière qui en fait le type de la famille des Orchidées, famille peu rich dans nos climats, mais qui, dans les pays chauds contribue pour beaucoup à l'ornement des forêts où elles vivent sur l'écorce des arbres. Là elles af. fectent les formes les plus étranges : il y en a qui res semblent à un papillon, à une amphore, à un sabot à une sauterello. 


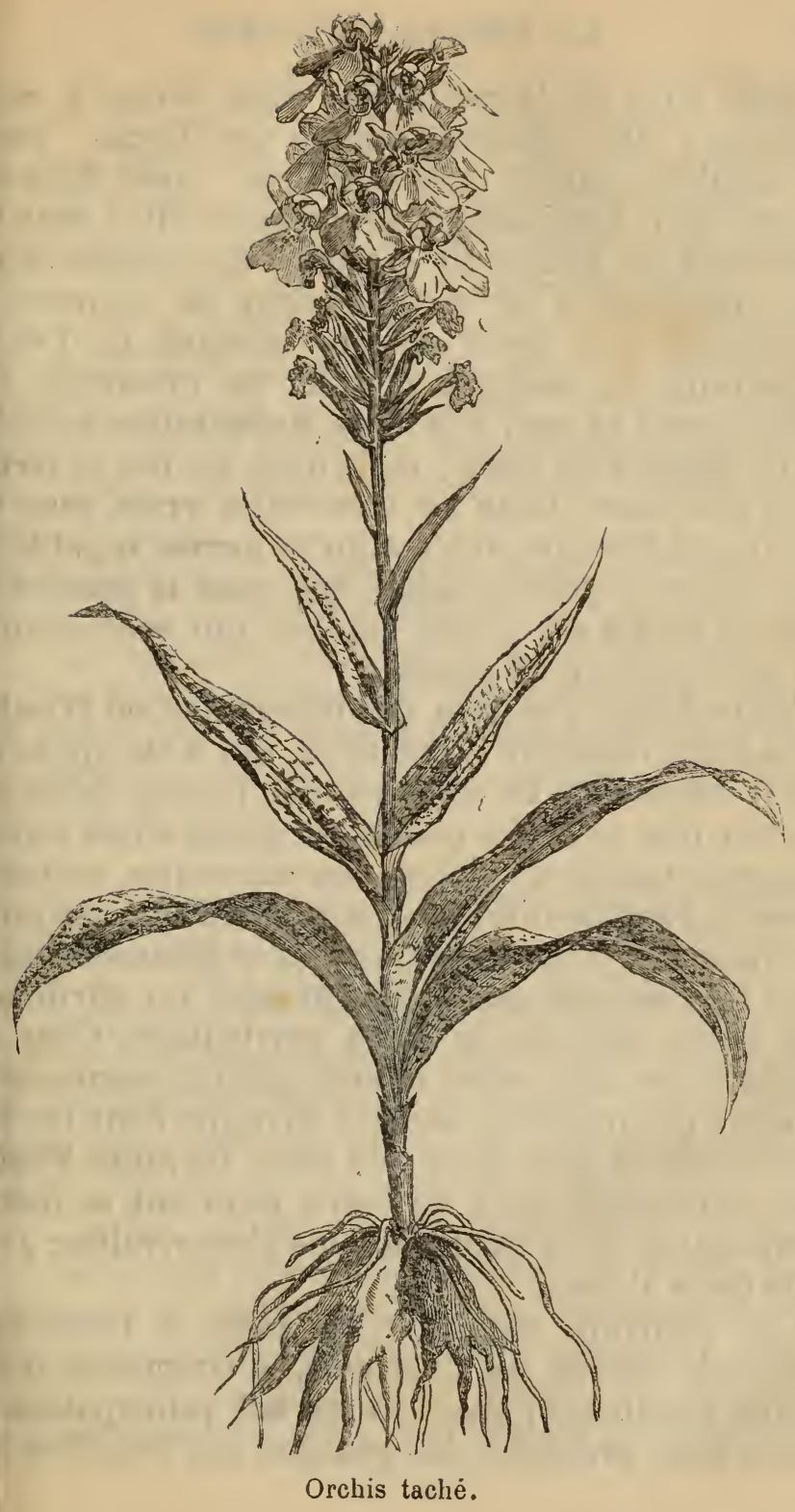


Mais c'est de la racine que nous avons à nous occuper; elle présente tantôt une forme ovale et régulière, tantôt celle que vous voyez dans cet échantillon. Les racines proprement dites sont les filaments qui entourent ces deux tubercules charnus, réceptacles de matières dont se nourrira la plante qui doit sortir de leur sommet. Le Dahlia nous offre un exemple familier de tubercule. On donne aussi ce nom aux tiges souterraines renflées de la pomme de terre; mais dans ce cas le terme n'est pas exact. Dans les tubercules vrais, ceux du Dahlia, de l'Orchis, il n'y a qu'un germe capable de reproduire la plante, tandis que dans la pomme de terre il y en a un grand nombre, qui sont comme les bourgeons d'un rameau.

Vous voyez que l'un des tubercules de l'Orchis est à demi desséché : c'est celui qui a été appauvri par la végétation de cette année; l'autre, destiné ê nourrir une nouvelle plante, est gorgé d'une espèce de gomme mêlée d'amidon. Ces tubercules, mondés. passés à l'eau bouillante, séchés et réduits en grain sous la meule, constituent le salep, substance alimen taire très-estimée des Orientaux, ,qui lui attribuent une vertu restaurante toute particulière. C'est $d f$ l'Orient que nous vient le vrai salep du commerce produit par diverses variétés d'Orchis dont les tu. bercules sont plus gros que ceux de notre Pente. côte; cependant ceux de notre pays ont la même composition, et le salep indigène peut rivaliser aver selui de la Perse.

On pourrait employer le salep à l'extérieur comme la racine de Consoude, la Guimauve et les autres émollients; mais on en fait principalemen usage pour préparer dess potages, des bouillies lé. 
res, qui conviennent dans le commencement de convalescence des maladies graves, parce qu'ils at d'une digestion facile. Mais il faut bien prendre rde de croire, ainsi que les marchands essayent le persuader, que le salep soit comme une quinsence d'aliment, de sorte qu'une très-petite dose ffise pour un repas. Il faut 60 grammes de lait ou bouillon pour dissoudre un gramme de salep, uel n'a pas plus de propriétés nutritives qu'un lange à parties égales de semoule et de gomme, lange que l'on vend quelquefois, par fraude, sous nom de salep.

Cette substance serait donc pour l'homme en nne santé un aliment insuffisant et d'un prix exsif. Le médecin l'emploie avec succès dans les ladies inflammatoires de l'estomac et des intess; mais c'est un remède de luxe, que l'on peut areusement remplacer par la gomme et la fécule pomme de terre.

Pouvez-vous me dire, mes amis, quelle est cette nte? - C'est une Citrouille. - Un Potiron. Pépon. - Une Courge. - Vous avez tous rai: la Citrouille (Cucurbita pepo) porte tous ces ns. Si ce végétal nous était moins familier, nous us étonnerions de voir un fruit si volumineux duit par une tige si mince. Connaissez-vous utres plantes qui ressemblent à celle-ci? - Le lon. - La Gourde. - Le Concombre. - C'est $i$; toutes ces plantes se ressemblent d'une mare frappante, et constituent une famille : celle des curbitacées. Ce sont des herbes annuelles, ramtes ou grimpantes, à tige creuse et rude. Les illes afternes, grandes, et couvertes de poils, sont 
fortement crispées. A l'aisselle de chaque feuille se développe un long filament appelé vrille, parce qu'il se contourne naturellement en spirale pou embrasser les branches des arbres, les treillis, e s'y accrocher avec assez de force pour soutenir le:

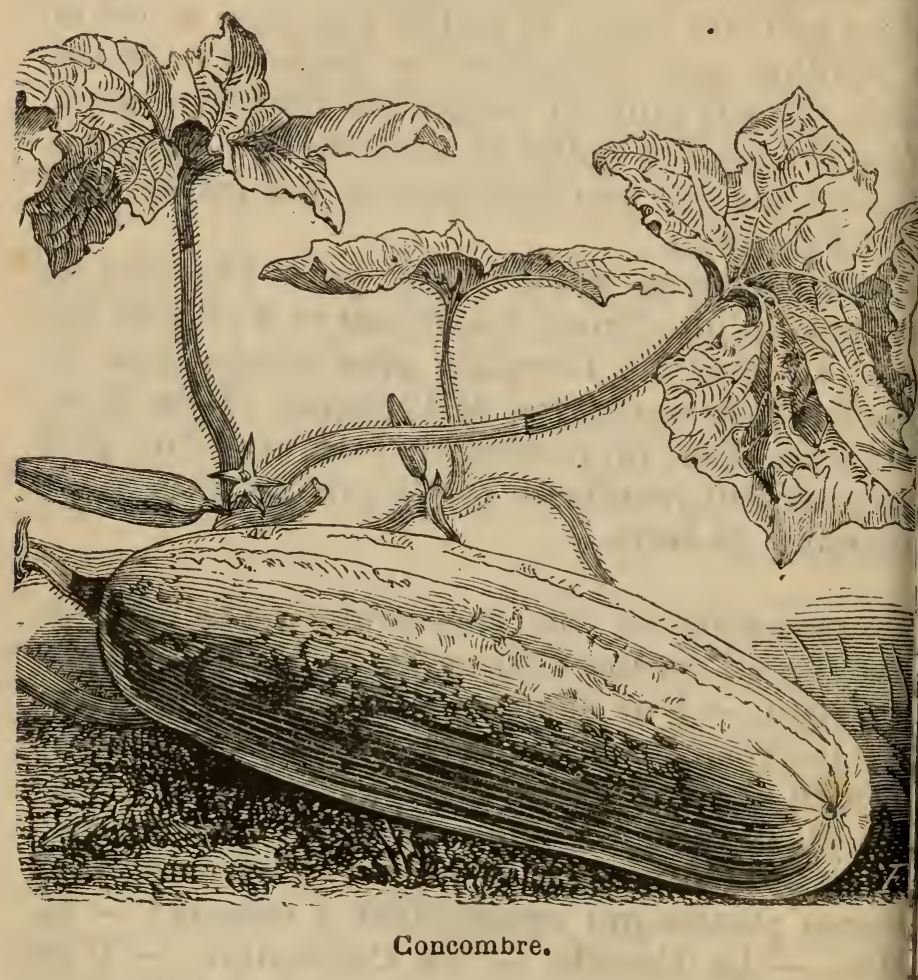

fruits pesants qui pendent de distance en distancefus Arez-vous remarqué ces fleurs de deux espèceles qui partent aussi de l'aisselle des feuilles ? Les unetoi sont petites, et passent presque inaperçues; les aupes tres forment un grand cornet jaune à cinq dents. $\mathrm{s}^{\mathrm{e}}$ 
détruisait sur un pied de citrouille les unes ou autres de ces fleurs à mesure qu'elles apparaist, la plante serait stérile; elle ne porterait pas fruits. Pour qu'une citrouille se développe à la ce de la grande fleur, il faut qu'une fine pouse née sur la fleur la plus petite lui soit apporpar le vent ou par un insecte, qui passe de l'une autre. Vous expliquer ce mystère de la nature is entraînerait trop loin aujourd'hui ; nous y rendrons une autre fois. Pour le moment, je veux lement vous parler des propriétés de la Citrouille. a pulpe de ce fruit, cuite, constitue pour l'homme aliment aqueux, rafraîchissant, et légèrement atif. Crue, on l'emploie avec succès, comme la mme de terre râpée, pour les brûlures qui n'ont pénétré au-dessous de la peau. Vous savez que Citrouille est d'une grande ressource dans une me. Les porcs s'en trouvent fort bien, ainsi que vaches, dont elle augmente le lait.

es semences ne sont pas moins utiles que la pe; elles contiennent environ le tiers de leur ds d'huile comestible et propre à l'éclairage. yées avec du lait ou de l'eau gommée et sucrée, s forment une émulsion émolliente et rafraîssante. Cette émulsion (préparation liquide dans uelle se trouve intimement mélangé un corps s) se recommande, en outre, par un mérite tout cial, celui de tuer le ver solitaire, dont il est rdinaire très-difficile de se débarraser. Il y a ax siècles que cette vertu était connue de queles guérisseurs des campagnes, sans que la méine eût pris soin de la constater et d'en répandre sage ; mais enfin ce remède indigène nous ayant envoyé comme nouveau de l'île de France, puis 
de l'île de Cuba, avec le prestige dont s'entoure qui vient de loin, a reçu l'approbation de plusieu médecins, et produit des cures nombreuses. Vo voyez combien il est difficile d'être prophète en si pays.

Nous resterons aujourd'hui dans les limites notre enclos. Le jardin va nous fournir assez plantes utiles pour vous intéresser. Vous les co naissez par leur nom, quelques-uns de leurs usag vous sont déjà familiers, mais vous ignorez sal doute la plupart de leurs propriétés.

Le Thym de cette bordure montre, par ses éclai: cies, que la bonne ménagère lui fait de fréquent visites.

Comme vous voyez, c'est une petite plante hau de quinze à vingt-cinq centimètres, très-rameust dont les feuilles opposées, c'est-à-dire placées en ri gard l'une de l'autre, à la même hauteur, sont rot lées sur les còtés, ce qui les fait paraittre très-étroites elles sont couvertes, en dessous, d'un duvet qu donne à la plante un aspect grisâtre. Les fleurs qui se succèdent de juin à octobre, sont roses, pur purines, et quelquefois blanches, arrangées à per près en épis.

Le Thyм (Thymus vulgaris) est employé en cui sine comme condiment, pour relever le goût der viandes fades ou de certains légumes. Placé dan les malles, les armoires, il en éloigne les mitei et autres insectes. Les abeilles lo recherchent 8 il communique à leur miel un arome assez pro. noncé, 
ssons maintenant en revue ses propriétés mées. Il est éminemment excitant, stimulant, c'est- qu'il donne impulsion au me nerveux, mble augmena vitalité. On loie avec sucson infusion les cas de pade l'estomac, aiblesse généaccompagnée ristesse et de que d'énergie, la colique et laux de tête.

ne infusion entrée, em. es en lotions ientes, au mod'une brosse eu rude, peut re pour faire - le petit inde la gale. oduit aussi de effets, en lo, et surtout en gations, dans ques cas de eurs nerveuses

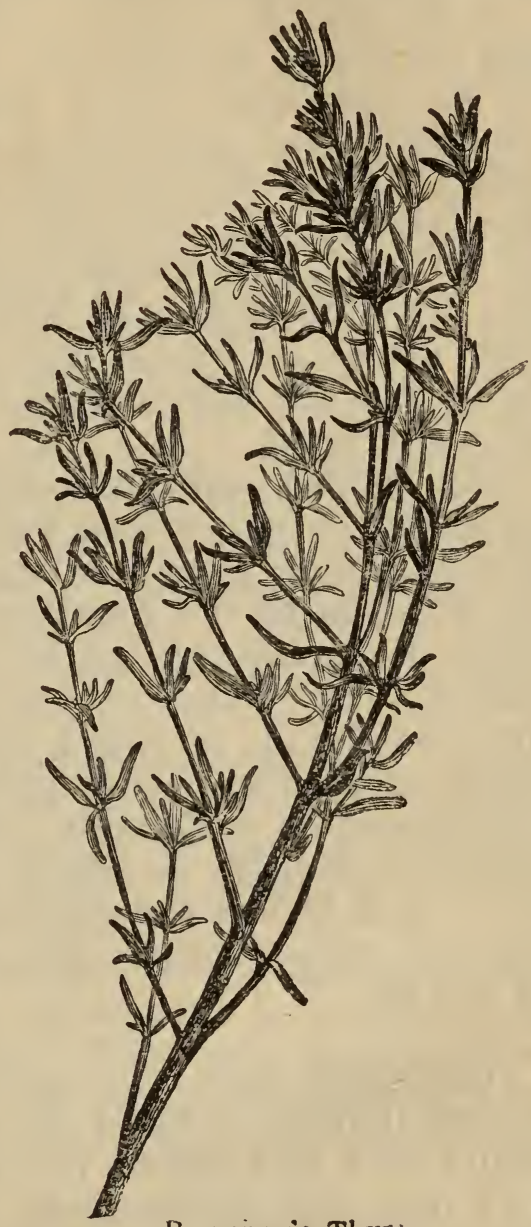

Brancie de Thyro.

humatismales des membres, Les bains chands, atisés avec du Thym, conviennent aux enfants 
scrofuleux ou très-délicats; on peut en mettre $q$ ques branches dans la paillasse de leur lit.

Le Serpolet (Thymus serpillum), si commu long des fossés, des chemins, et dans les pelor des bois secs, n'est qu'une variété du Thym l'on cultive dans les jardins et qui croît sponte ment dans nos départements du Midi. Il poss les mêmes qualités, mais à un degré un peu mc dre. Le Thym et le Serpolet donnent, à la disti tion, une huile essentielle, très-fragrante, dont petite goutte, introduite dans une dent cariée, moyen d'un peu d'ouate, peut calmer pour quel temps la douleur.

Cette humble petite plante a donc quelque més et l'on doit la trouver dans tous les jardins. Si v avez des ruches, plantez-en le plus que vous po rez. Je me bornerai à vous dire aujourd'hui qu' est de la famille des Labiées, dont nous auri souvent à nous occuper, car beaucoup de ses me bres possèdent à un degré remarquable les $p$ priétés stimulantes, dues principalement à le parfum, comme le Romarin, la Menthe poivrée, Mélisse, la Sariette, le Basilic. Il faut récolter Thym après la floraison, c'est-à-dire dans le cour d'octobre, et le suspendre par petits paquets da un lieu sec.

Vous savez tous, sans doute, reconnaître Laurier (Laurus nobilis), qui s'élève quelquef jusqu'à six à sept mètres, l'un de nos arbres to jours verts, aux feuilles dures, coriaces, foncées lisses en dessus, un peu plus pâles en dessous. S fleurs, petites, d'un blanc jaunâtre, se réunisse en petits faisceaux ou corymbes, comme disent 1 
nistes, disposition dans laquelle elles forment espèce de parasol à rayons un peu inégaux. A fleurs succède un fruit de la grosseur d'un n de cassis et qui devient bleu en mûris-

arbre fut dédié par les Grecs à Apollon, dieu arts, d'où lui vient son nom de Laurier des es; on l'appelle aussi Laurier franc. Jadis une onne de Laurier était la récompense des vainirs, des héros; plus tard elle devint une dision académique, d'où le mot lauréat ; aujouri on la fait encore figurer sur la tête des souins dont l'effigio est gravée sur les pièces de naie. Malgré ces illustres emplois, comme les ons-bleus de tous degrés font entrer ses feuilles le bouquet destiné à relever le goût de leurs arations, le Laurier noble a été baptisé démoquement Laurier sauce, ce qui ne lui retire de sa valeur.

appartient à la famille des Lauracées; qui est ssez répandue dans les régions intertropicales, omprend les végétaux les plus aromatiques. lui devons la Muscade, la Cannelle, le Sassale Benjoin, le Camphre.

Laurier possède des vertus stimulantes, qu'il à ses principes balsamiques. Il convient donc les affections qui affaiblissent les organes et ent les fonctions languissantes, tandis qu'il est e-indiqué toutes les fois qu'il y a irritation ou mmation.

s feuilles, âcres au goût, sont aromatiques, des. Leur infusion est utile en lotions pour aler les ulcères; en bains, pour raffermir les $s$ et fortifier les enfants délicats. On en extrait 
une huile essentielle avec laquelle on pratique frictions dans les névralgies, le rhumatisme, la ralysie restreinte. On retire aussi de ses baies fruits une huile volatile analogue à celle des fe les, connue sous le nom d'huile de Laurier, et est utile pour exciter les tumeurs molles, résov les engorgements.

On brûle souvent des feuilles de Laurier ou d tres végétaux aromatiques dans la chambre des lades, sous prétexte d'en purifier l'air. Mais l'od agréable qu'elles répandent ne peut que dissim! celles qu'on désirait faire disparaitre. C'est le de tous les prétendus désinfectants, à l'exception chlorure de chaux. Au lieu de brûler du sucre, vinaigre ou des aromates dans la chambre d malade, n'y laissez jamais l'air se vicier, faites sorte qu'il se renouvelle constamment, et en d'épidémie, maintenez-y, dans une assiette, un de chlorure de chaux humide.

Arrêtons-nous devant cette belle touffe de plar herbacées, hautes de plus d'un mètre, à grosses ges cylindriques, fistuleuses, c'est-à-dire crec en partie, et couvertes d'une poussière glauc Voyez ces grandes feuilles profondément décour en deux ou trois lobes, dentées, d'un beau veri dessus, blanchâtres en dessous, et soutenues su tige par un pétiole fistuleux qui s'élargit beauc vers sa base. C'est l'Angélique (Angelica arch gelica).

Vous vous rappelez sans doute avoir vu, pend l'été, ses fleurs verdâtres, petites et nombreu: disposées en parasol à rayons égaux, formé même de petites touffes arrangées avec la me 
l'inflorescence offre ce caractère distinctif form une nombreuse et très-importante famille, celle Ombellifères. Do ce nombro sont lo Panais, le I sil, la Carotte, la Ciguë, le Céleri.

L'Angélique doit son nom à l'odeur balsami de ses feuilles. Vous pouvez vous assurer de sa veur aromatique, un peu âcre et amère. La rac: qui est la partie la plus employée en médecine, d'abord douceâtre, puis chaude, aromatique, am et provoque la salivation.

A ces caractères, vous reconnaissez qu'elle avoir des propriétés stimulantes énergiques.

Les feuilles d'Angélique perdent leurs vertus la dessiccation, aussi n'emploie-t-on en méder que les graines et la racine. Cetto partie est la ] importante. On la récolte en automne. Après l'al nettoyée, on la fend en morceaux, on la sèch on la renferme dans des boîtes.

L'infusion se prépare avec une once de ra dans un litre d'eau. C'est un des stimulants les utiles, principalement pendant la convalesce longue et difficile des maladies graves. Elle ex doucement le système nerveux et combat la tor de l'appareil digestif. Les jeunes tiges fraîches vent s'employer à la place de la racine. Les pétil confits au sucre et la liqueur produite par la cération des tiges dans de l'eau-de-vie, possèd sous une forme très-agréable, toutes les qua médicales de la plante.

L'Angélique croît naturellement sur nos mo n gnes et prospère jusqu'en Laponie. Il ne faut pn la confondre avec l'Angélique sauvage qui sur le bord des fossés et des ruisseaux, dans bois, les lieux ombragés et les prairies humi 
lle-ci est notablement plus petite et douée de opriétés plus faibles. On peut toutefois l'employer, ute d'Angélique vraie, on ayant soin d'augmenter ; doses.

Les peuples des contrées boréales, dont la flore $t$ bien moins riche que la nôtre, et qui ont fait $s$ rares végétaux de leurs climats une étude praque des plus fécondes, estiment fort l'Angélique. a Norvége, en Islande, en Sibérie, on l'emploie mme aliment et comme condiment; on mange ec du pain les jeunes tiges crues dépouillées de ur épiderme. Les Norvégiens mettent un peu de cine d'angélique dans le pain. Les Lapons l'emoient dans la colique, dans les affections de poiine. Ils mâchent sa racine, et croient que cette ante a la propriété de faire vivre longtemps. Un mmé Camous, qui mourut à Marseille à l'âge de 1 ans, avait l'habitude de mâcher de la racine Angélique, et comme les Lapons, il attribuait à s vertus stimulantes, toniques et restauratives, n étonnante longévité.

Maintenant que vous savez ce que c'est qu'une ante Ombellifère, voyons si vous pourrez m'en gnaler quelqu'une dans ce jardin. - Voici en fet deux bons échantillons, d'autant plus qu'ils partiennent à la classe de remèdes dont nous cauns aujourd'hui : celui-ci est l'Anss (Pimpinella isum), l'autre, le Fenoull (Anethum foniculum). n les désigne aussi sous les noms d'Anis bouge, Anis vert, Boucage à fruits sauvages ; Fenouil es vignes, Anis doux, Aneth, Aneth-fenouil. Elles frent beaucoup de points de ressemblance. Ce ont dos vógétaux herbacés : lo premior, annuel; le 
second, vivace; à tige fistuleuse, remarquables $\mathrm{k}$ leur feuillage divisé en lanières étroites, principa ment vers le sommet de la plante. Ce que l'on a pelle communément la graine de l'Anis et du F nouil sont en réalité de petits fruits. Brisez un ceux de l'anis, et vous y verrez les véritables gri nes, grosses comme une tête d'épingle.

Ces fruits ou graines exhalent une odeur arom tique assez intense, leur saveur est d'abord un $p$ sucrée, puis chaude et stimulante. Le principe ac réside dans une huile essentielle, très-abondan qu'on en sépare par la distillation.

L'odeur d'Anis se retrouve, à divers degrés, da un assez grand nombre de végétaux appartenan des familles très-éloignées, croissant sous tous climats. Eh bien! les indigènes de ces pays ont pa tout découvert les propriétés inhérentes à cet odeur, et emploient depuis longtemps ces plant comme stimulantes et toniques.

Les vertus du Fenouil et de l'Anis sont les même. Il n'y a pas dans la médecine domestiqne, de r mède plus efficace contre l'atonie de l'appareil c gestif, les flatulences. On les emploie journelleme pour combattre les coliques venteuses, et l'ensemk de symptômes pénibles qui accompagnent la dy pepsie, maladie dans laquelle l'estomac et les i: testins, devenus paresseux, accomplissent lent ment et imparfaitement leurs fonctions, d'où $r$ sultent au bout de peu de temps des désordr graves, faiblesse générale, maux de tête, tristes et dégoût des aliments. Mais rappelons que des c liques accompagnées de fièvre, des douleurs d'e tomac causées par une inflammation réclament $d$ émollients et non des stimulants. Administrés 
reils cas, ceux-ci ne feraient qu'aggraver le mal. ne suffit donc pas de dire : l'Anis est bon pour la lique; il faut remarquer qu'il est seulement utile ins les cas où les intestins, par suite d'une faiesse maladive, ne peuvent pas se débarrasser des z qui les distendent. Mais si la colique provenait l'irritation, de l'inflammation des parties, au lieu un stimulant, il serait nécessaire d'employer une ane de Chiendent, de Consoude, ou des lavements amidon, c'est-à-dire des remèdes émollients.

A côté des deux échantillons d'Ombellifères que us m'avez apportés, je trouve celui d'une plante i n'est pas de cette famille. Nous n'allons pas ins l'examiner. Ce qui a contribuéà vous induire erreur, c'est la disposision du feuillage découpé ofondément en lanières presque filiformes. Mais fleurs de celle-ci sont disposées d'une façon trèsférente, chacune d'elles forme ce que l'on appelle capitule, c'est-à-dire une réunion de plusieurs tites fleurs très-simples, ce qui leur a fait donner nom de Composées. La grande Marguerite des és en est un exemple. Ce que vous appelez une iille dans la fleur de Marguerite est en réalité une ur entière.

Cette plante qui nous occupe, c'est l'Aurone ( $A r$ nisia abrotanum), appelee aussi Armoise mâle, moise des jardins, Citronnelle, Armoise citronle. Ces derniers noms viennent de ce qu'il suffit froisser légèrement ses feuilles pour développer o odeur de citron assez prononcée, fraîche et exante.

On peut cueillir pendant toute l'année les raaux de l'Aurone pour les conserver. La dessic- 
cation ne diminue pas sensiblement ses propriét C'est encore un médicament stimulant; son infusic d'un goût très-agréable, est utile contre les ven stomachique et légèrement anthelminthique, c'e à-dire capable de faire périr les vers intestinaux

Vous avez dû remarquer, mes amis, que $p$ classer une plante, c'est-à-dire pour détermine. quelle famille elle appartient, j'ai d'abord exam ses fleurs. Ce sont elles, $\bullet n$ effet, qui forment base de la classification des végétaux par famill Une des choses les plus intéressantes pour vo est donc de bien savoir ce que c'est qu'une fle et de pouvoir reconnaître les différences essent les qui les distinguent, aux yeux du botaniste, $b$ plus que leur couleur, leur taille ou leur parfum

La fleur est l'ensemble d'organes destinés à p duire un fruit, une graine, c'est-à-dire, à assurer reproduction de la plante.

Prenons-en une, un peu grande, celle de la pucine, par exemple : nous en avons beaucoup sc la main, et vous pouvez les cueillir pour suivren explications.

En regardant d'abord cette fleur par derriè vous voyez, insérée sur la tige, une envelol en forme de cornet recourbé ou d'éperon, $t$ minée à la partie supérieure par cinq dentelu aiguës. Cette première enveloppe s'appelle cali Elle est verte dans la plupart des fleurs, mais qu quefois elle se colore au point de ressembler à fleur même, comme nous le voyons ici. Bien c sa forme soit très-variable, le plus souvent elle toure la fleur d'une espèce de collerette dentelée Enlevons avec précaution le calice. Voici, 
mbre de cinq, ce que vous appelez les fcuilles de fleur; en botanique, on les nomme pétales. Leur mbre varie extrêmement. Il y a des fleurs qui

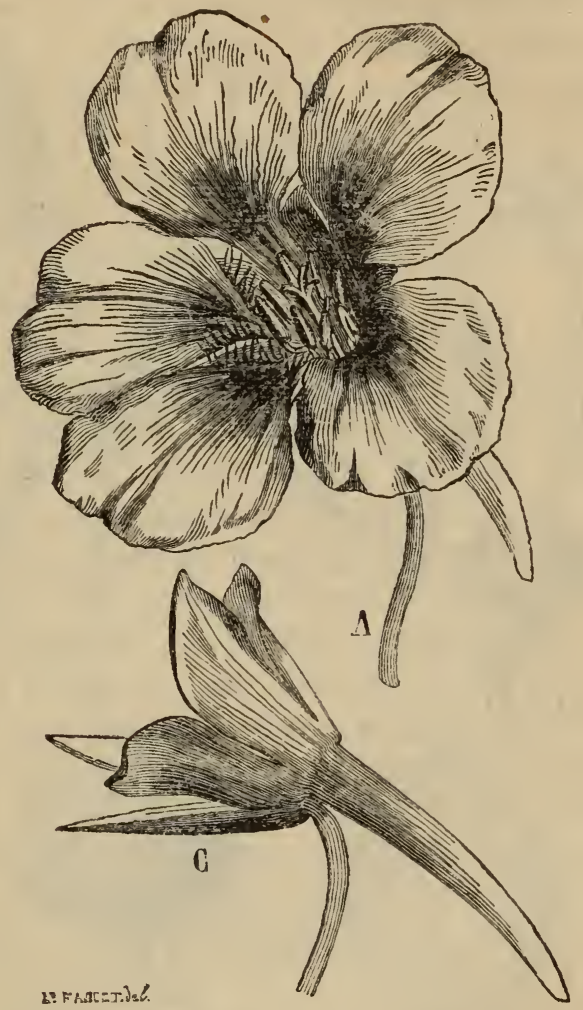

Capucine. is fleur. C, le calice et son éperon.

n ont point: d'autres, comme les roses doubles, offrent cinquante, soixante ou davantage. Vous s étonnés do m'entendre dire que certaines fleurs nt point de pétales; car pour vous, leur réunion, 3 nous appelons corolle, constitue la fleur. Co 
ne sont pourtant que des accessoires, si brillant qu'ils puissent être. Ce qui constitue véritablemen une fleur, nous lo trouvons en arrachant un à ut

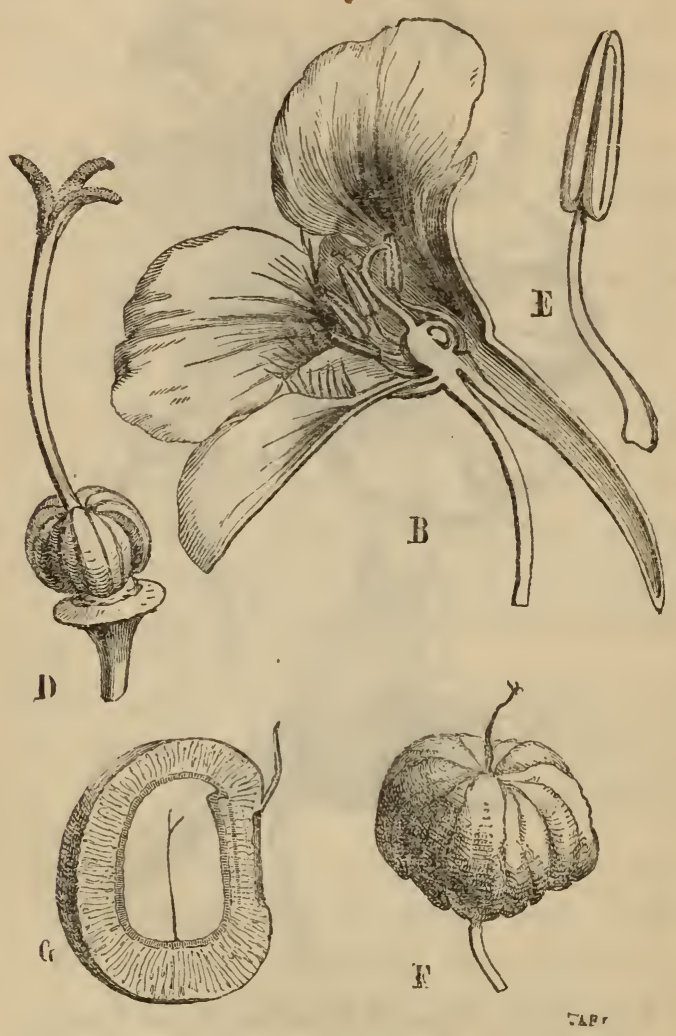

Capucine. B, conpe verticale de la fleur. D, le pistil montrant les différentes parties, ovaire, style et stigmate. $\mathrm{E}$, une étamine. F, fruit . G, coupe du fruit.

les pétales. Ce qui reste suffit pour reproduire 1 . plante, c'est la fleur; le calice, les pétales, ne son que des enveloppes protectrices. Remarquez ce 
it petites masses ovales soutenues par de minces ets : ce sont les étamines; lorsqu'elles sont arées à maturité, elles s'ouvrent et laissent échap- une fine poussière qu'on appelle pollen. Celui la fleur de lis, abondant et d'un beau jaune d'or, us a fourni l'occasion de plus d'une espièglerie. tachons les étamines. Il nous reste une tige diée à la partie supérieure, renflée à sa base, c'est pistil. La partie supérieure s'appelle stigmate; tige, style; la base, ovaire.

Jne étamine, un pistil et un ovaire sont nécesres à la reproduction de la plante; mais il peut faire qu'une fleur n'ait que le pistil, par exem, tandis qu'une fleur voisine n'a que l'étamine. st ce qui a lieu pour la Citrouille.

L'étamine constitue l'organe mâle ; le pistil, l'orne femelle. Voyons comment ils produisent le it ou la graine. Le pollen tombe sur la surface iante du stigmate, s'y attache, et lance un filant qui, traversant le stylt, arrive dans l'ovaire, pénètre dans un germe, un petit œuf de plante, n de le féconder, c'est-à-dire de lui donner la priété de se développer. L'ovaire commence alors roître, et quand on voit cet organe grossir après chute des fleurs d'un arbre à fruits, on dit que fruits sont noués.

voilà ce qu'il vous est le plus essentiel de savoir sujet de la fleur. Les mots nouveaux que j'ai ployés vous deviendront familiers par l'habitude me les entendre répéter. Ne vous découragez , s'ils vous échappent pendant quelque temps. Maintenant, revenons à nos plantes. Et, puisque is avons entre les mains des fleurs de Capucine, fitons de l'occasion pour en dire quelques mots. 
Originaire du Pérou et du Mexique, où elle es vivace, la CAPucine (Tropæolum majus), nommé

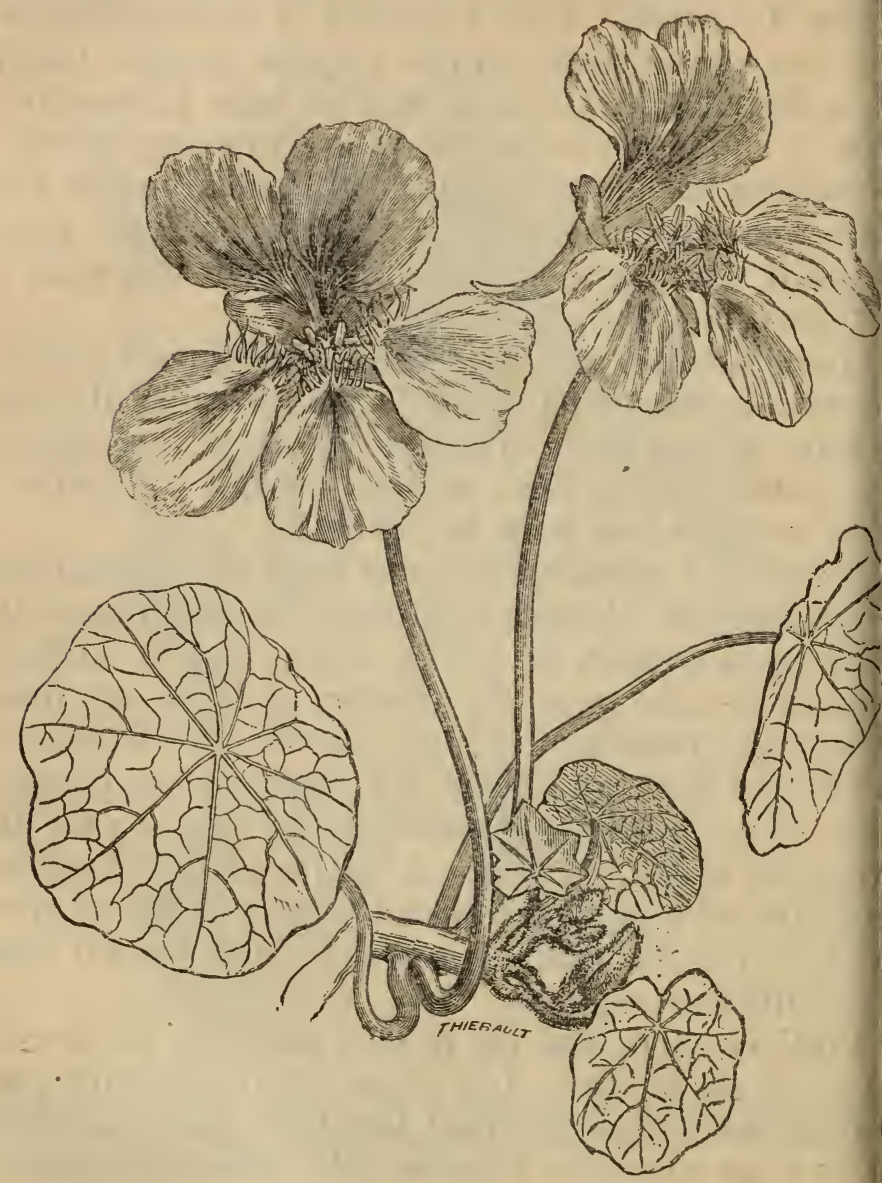

Capucine.

aussi Cresson d'Inde, est une de nos plus jolie plantes grimpantes, qui fleurit jusqu'aux première 
es. On mange ses fleurs en salade, et l'on confit inaigre les boutons et les fruits; mais on ne pas assez usagge des feuilles à saveur piquante, eu poivrée. Toutes les parties de la plante sont ulantes, et peuvent rendre do grands services les cas de scrofules et de scorbut. La Capuest de la famille des Géraniacées, dont nous ns occasion de parler dans la suite, qui a pour le Géranium.

fleur de Capucine donne lieu à l'un des phéènes les plus curieux que l'on ait lieu d'obserdans les végétaux. Pendant les jours les plus ds de juin, si vous observez, au crépuscule du n ou du soir, la variété rougeâtre, vous la verprojeter, de temps à autre, de petites étincelles. it la fille du botaniste Linné qui appela d'abord ention sur ce fait. On a, de nos jours, découdu phosphore dans la fleur de Capucine; tous sa présence ne semble pas expliquer le phéène des étincelles, qui ne ressemble en rien à osphorescence $d u$ bois pourri, mais bien à la arge d'une petite machine électrique. On consquelquefois, la production d'étincelles sur tres fleurs de la même couleur, l'Eillet d'Inde, s orangé et lo Souci, plante trop dédaignée, à elle j'ai offert, vous voyez, une large hospitaNe passons pas près de lui sans nous inforde ses vertus.

Souar (Calendula officinalis), de la famille des osées, est une de ces plantes qui semblent amies homme, tant elles se reproduisent facilement ur de lui. Sans parler du Souci des champs, ouci de vigne, celui de nos jardins s'y main- 
tient sans culture et donne pendant plusieurs mo ses belles fleurs orangées, qu'on dédaigne pari qu'elles sont trop faciles à obtenir.

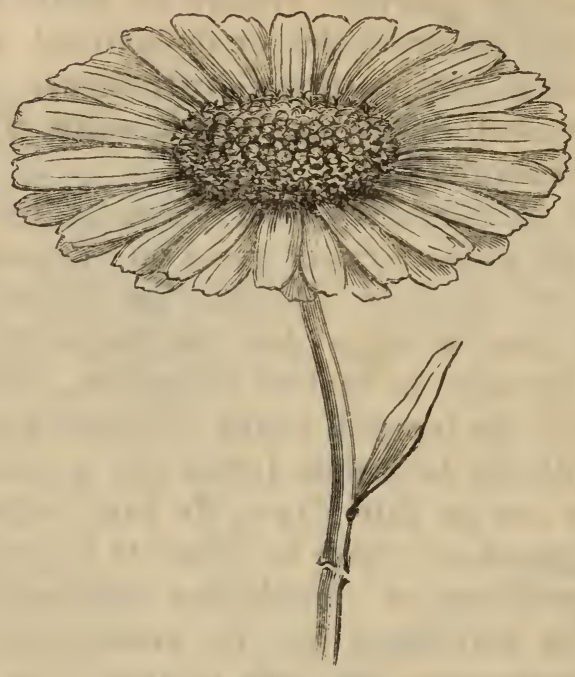

Fleur du Souci.

Toute la plante exhale une odeur aromatiq pénétrante, un peu bitumineuse; la saveur amère et âcre, surtout dans la racine et dans feuilles. A ces signes, on peut lui supposer to d'abord des propriétés stimulantes, excitantes. EI appartient en effet à la classe de remèdes sur le quels j'appelle aujourd'hui votre attention, et $q$ nous occupera souvent dans nos promenades.

On doit toujours employer les boutons, fleurs sommités de tiges à l'état frais; en séchant, ils d viennent inertes. Les feuilles fraîches, écrasées, $m$ difient d'une façon favorable les tumeurs et les u 
ns scrofuleuses, surtout si l'on prend en temps une infusion des boutons ou des fleurs. גilles écrasées, appliquées avec persévérance uemment renouvelées, peuvent faire tomber rues, les cors et les durillons. Enfin, comme excitant général, le Souci favorise l'excrétion ines et de la sueur. Voilà certainement des suffisants pour l'admettre dans notre col-

terminer notre promenade, arrêtons-nous cette belle plante vivace à tiges simples, cyues, haute de 50 à 60 centimètres, et dont vez admiré pendant l'été les fleurs blanches pées, surmontées de longues étamines, et disen épis. C'est la Fraxinelle (Dictamnus de la famille des Rutacées, désignée sous ms de Dictame blanc et de Buisson ardent. m ordinaire vient de la ressemblance de son ge avec celui du frène. Les feuilles, qui sont es comme celles-ci de la réunion de plusieurs feuilles sur un même pétiole, s'appellent bsées.

ous frottez légèrement une de ces feuilles, il égage une odeur pénétrante, aromatique, anaà celle de la Citronnelle; les fleurs sentent hent le citron. Cette odeur peut faire préjuger priétés de la plante : nous sommes autorisés 'abord à la supposer stimulante, excitante et chique, comme toutes celles que nous avons es aujourd'hui. Le thé de Fraxinelle, d'un journalier en Sibérie, est aussi agréable que le l'Aurone, et possède les mêmes vertus. corce de la racine, qui exhale une odeur de 
houc assez prononcée, était jadis vantée pour $\mathrm{g}$ rir la fièvre, les scrofules, la peste, les fièvres termittentes. Il est certain qu'elle peut rendre services comme tonique stimulant; mais les rés tats obtenus sont trop peu décisifs pour en ff recommander l'usage. Cependant on la recue encore à l'automne, pour la livrer au commerce.

Vous avez été surpris, sans doute, quand je vi ai dit que la Capucine et quelques autres fleurs d rouge orangé devenaient, pendant les nuits chau et sèches de l'été, de petites machines électriq d'où partaient des étincelles lumineuses. La Frt nellc donne lieu, dans les mêmes circonstance: un phénomène non moins intéressant. Nous savi que le parfum des plantes est dù à une huile sentielle volatile. Dans la Fraxinelle, cette huile sécrétée en abondance par de petites glandes nc breuses surtout sur les sommités. Par un ten sec et chaud, cette huile s'évapore lentement, manière à former autour de la plante une atm phère artificielle. Si on approche une allumette flammée, cette atmosphère prend feu et brûle pi dant quelques secondes en développant une v flamme. Voilà pourquoi la Fraxinelle s'appelle $\mathrm{B}$ son ardent.

Voici dans la prairie une de ces plantes activ vénéneuses même, qu'il faut se garder d'adj nistrer sans l'avis du médecin, mais qui, entre mains habiles, peuvent produire les plus heure résultats : c'est le ColchiQue D'Automne (Colchici autumnale), qui sert de typo à la famille des $C$ 
acées. Il a plusieurs noms vulgaires : Safran prés, Safran bâtard, Mort-chien, Tue-chien, leuse, Veillotte, Chenarde, etc.

Colchique est une plante vivace, herbacée, consiste en un ognon ou, pour parler comme otaristes, un bulbe d'où sortent, en automne, ou quatre belles grandes fleurs d'un lilas tenallongées en tube et découpées en six divisions is, un peu aiguës. A ces fleurs succèdo un ou capsule, divisé en trois compartiments lis de graines, qui mettent près d'une année à $r$. Les feuilles n'apparaissent pas en même $s$ que les fleurs, mais se développent au prins suivant. Elles sont grandes, lancéolées, c'este ovales et terminées en pointe comme une Les animaux n'y touchent jamais dans la ie, et s'ils en mangent, à l'étable, mêlées à res herbes, ils sont atteints d'une inflammation ntestins qui peut causer la mort. L'homme ive les mêmes accidents.

aut veiller à ce que les jeunes enfants ne mâpas les fleurs de Colchique, car ils pourraient e sérieusement incommodés. La médecine les pie fraîches, mais se sert principalement des es et des bulbes. Voyez celui-ci, que j'ai arravec soin ; un côté est arrondi ; l'autre, un peu , présente un sillon à la base duquel apparaît -me du nouvel ognon destiné à remplacer ceii a fleuri et qui a perdu ses propriétés médiC'est au mois d'août que le nouvel og'non est toute sa force, et c'est alors qu'il faut le cueilqui n'est pas facile, à moins que l'on n'ait ué d'avance sa place, car il n'y a alors ni es ni fleurs pour indiquer sa présence. Il n'y 


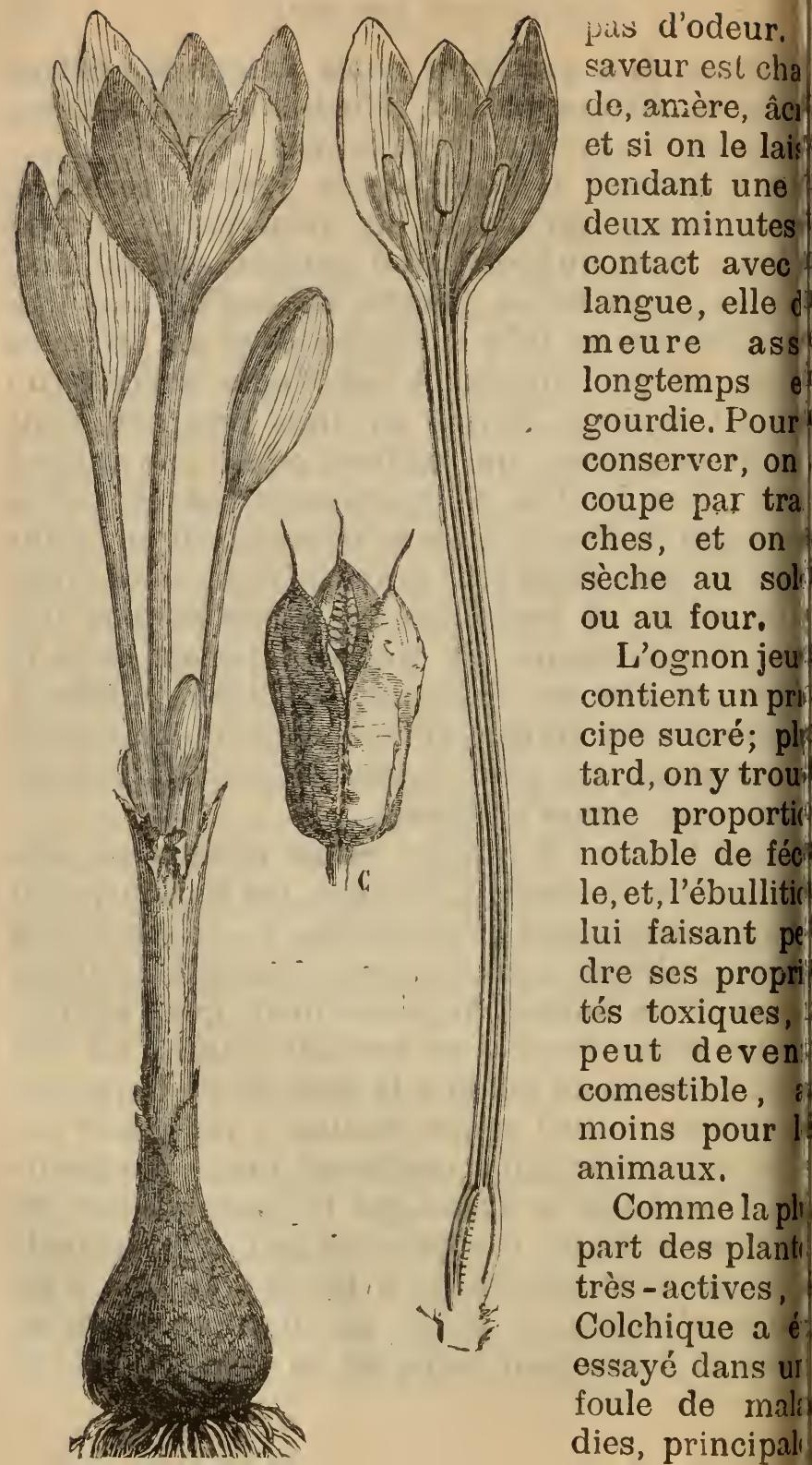


- lesquels la médecine est souvent impuisLes seuls résultats incontestables sont ceux us dans l'hydropisie, le rhumatisme et la e. Cependant il est rare qu'il guérisse radient ces affections, qu'il détruise la prédispoaux attaques futures; il calme la douleur lle, ce qui est déjà beaucoup, plutôt qu'il ne isparaître le germe de la maladie. Il agit à egrés divers, selon les individus, sur l'estoles intestins ou la vessie; mais on le consisurtout comme diurétique et apéritif.

deux mots demandent explication. On nomme ifs les médicaments que l'on suppose capale favoriser l'expulsion des matières dont la hce, dans le sang, donne lieu à des maladies, e la jaunisse, la goutte. C'est surtout par les que l'économie se débarrasse de ces substanantôt spontanément, tantôt sous l'influence de les propres à en augmenter la quantité, et que ppelle diurétiques. Les propriétés apéritives rétiques se rencontrent souvent dans le même : nous allons rechercher aujourd'hui des vé$x$ qui les possèdent. Toute boisson abondante rtout chaude augmente nécessairement la ité des sueurs ou de l'urine, puisqu'il lui faut er une issue; mais on doit réserver le nom de iques aux substances qui, par une action spéur les reins, les obligent à sécréter une urine bondante ou d'une composition anormale. ais vous dire deux mots d'une autre plante ique non moins active et dangereuse, la (Scilla maritima), de la famille des Liliaqui a pour type le Lis commun, Elle croit sur rds sablonneux de l'Océan et de la Méditerra- 
née. Son bulbe, gros comme les deux poi écailleux, produit d'abord un.9 hampe, puis de g. des feuilles lancéolées. Les fleurs, très-nombrev disposées sur la hampe en long épi, sont blanc avec une légère teinte de violet. On emploir écailles du bulbe, ayant soin de rejeter celles d surface et celles du centre.

Voici un arbuste d'apparence bien modeste bougri, couché, tortueux; on dirait qu'il a h de vivre, et ses longues aiguilles ne sont pas $t$ pour attirer la main. Heureusement nous sa qu'il ne faut pas se fier aux apparences. Les f] si engageantes du Colchique sont vénéneuses a peut-être un trésor dans ce buisson héris: bourru.

Cet arbrisseau vous est d'ailleurs familier : c' Genévrier (Juniperus communis), que l'on ap aussi vulgairement Genièvre, Potron, Pétrot.

Il appartient à la famille des Conifères, presque tous les individus nous fournissent produits fort importants. C'est déjà une bonn commandation. Le type de cette famille est le remarquable par ses feuilles simples, minces, tes, longues et aiguës, réunies en faisceaux dei trois ou cinq. Il porte pour fruits ce que vou peiez des pommes de pin, et ce que les botar nomment des cônes, d'où le nom de la fas Ceux-ci sont formés d'écailles épaisses et $d$ qui, après plusieurs années, se séparent et lail tomber la graine huileuse, féculente et comes Les Sapins, les Mélèzes, sont des Conifères Thuyas, les Cyprès, les Ifs, les Genérriers, to so classani dans la mêmo famille; s'en édoi; 
plusieurs caractères particuliers, entre autres orme et l'arrangement des feuilles. C'est à cette ille qu'appartiennent les plus grands végétaux nus. Il y a en Californie des espèces de Pins ts d'environ 130 mètres, et dont le tronc meo 9 mètres de diare. On a enlevé c soin l'écorce d'un cos géants pour en e, à San-Francisco, parois d'une salle concert qui con$t$, outre un piano, siéges pour quae personnes!

ais revenons à noarbuste. Vous le ez réduit à des protions rabougries, - qu'il ne refuse de croître dans mauvais terrains me celui-ci; mais on le plante en ne terre, à une sition bien chauil s'élève à 4 ou 5

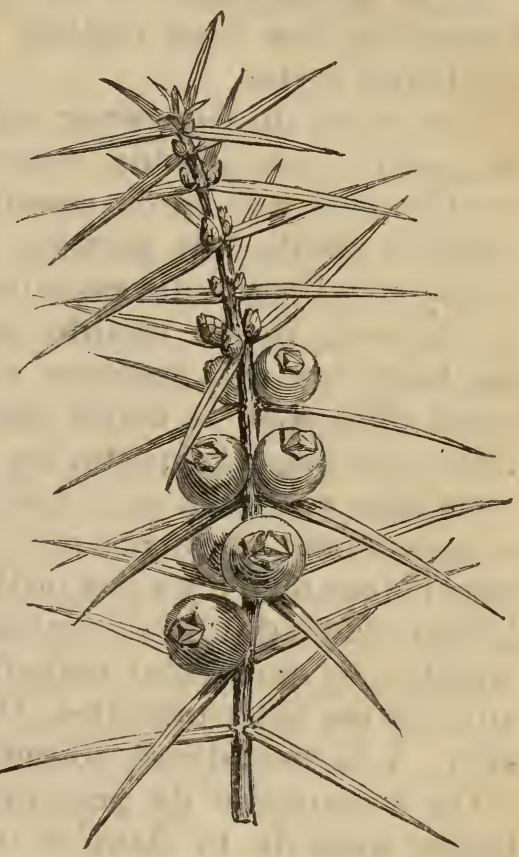

Ramoau de Genóvrier. res, et fournit un bois d'un grain serré, incorible, excellent pour fabriquer des seaux et tres ustensiles.

n reconnaît facilement le Genévrier à ses feuilinéaires, c'est-à-dire extrêmement étroites et ues, piquantes, fixées trois par trois à la tige. fleurs sont mâles et femelles, comme dans toute 
cette famille, composées de petites écailles. Le fr de la grosseur d'un pois, vert pendant deux ans brun à l'automne de la troisième année, époque sa maturité, ressemble à une baie charnue plv qu'à aucun des autres fruits des Conifères. Cep dant la partie pulpeuse est produite par la tra formation des trois écailles, bien accentuées d les fruits verts.

Ces baies du Genévrier ont une saveur d'ab douceâtre, puis chaude, amère et aromatique. E contiennent une huile essentielle abondante, d laquelle réside leur principe actif. Leur action nérale sur l'économie est stimulante; elle donne la vigueur, de la vitalité aux organes, et an les fonctions des surfaces sécrétantes. Voilà cc ment elles sônt si utiles dans les débilités de l, tomac, les engorgements, les maladies scrofuleu: mais elles semblent agir spécialement sur les re et constituent un de nos meilleurs diurétiques, $c$ tous les cas où il n'y a pas inflammation, mais ato Elles rendent de grands services dans les hydropi simples, et dans l'état maladif qui succède sou aux fièvres intermittentes. On les emploie en in sion, à la dose d'une poignée dans un litre d't

On a coutume de pratiquer des frictions e. tantes avec de la flanelle imprégnée des vapi qui s'exhalent des baies de genièvre projetées des charbons ardents. Quelques gouttes de l'h essentielle donneraient, avec moins de peine, résultat plus certain.

La décoction du bois réduit en copeaux, dose de 60 grammes par litre d'eau. est un sudorifique. On s'en sert également pour lave ulcères indolents. 
Les baies du Genévrier, infusées dans l'eau, donnt un Vin de Genièvre ou Genevrotte, qui est lubre et légèrement diurétique. Voici la manière procéder. Dans un hectolitre d'eau, on jette litres de baies et une poignée d'Absinthe; on sse fermenter pendant un mois environ, dans un droit frais, et l'on tire au clair. On peut pendant elque temps remplacer par de l'eau le vin que $n$ a retiré. Le vin de Genièvre fournit, par la dislation, une eau-de-vie aromatique qui conserve léger goût de térébenthine; mais le Genièvre commerce est d'ordinaire préparé en distillant l'eau-de-vie sur des baies, ou même en y ajout simplement un peu de leur huile essentielle. 'huile de Cade, employée en médecine vétérire, provient de la distillation du bois du Genévrier cèdre. C'est une substance très-active, que l'on doit confier qu'à des mains expérimentées. Tous voyez, mes amis, que l'humble Genévrier roit à quelque considération. Les propriétaires forêts, lo classant parmi les sous-bois do peu valeur, l'abandonnent d'ordinaire aux pauvres is, qui en font des bourrées : c'est un nouveau vice qu'il leur rend, après leur avoir fourni des ssons salubres et des remèdes précieux.

loyons, mon ami, ce que vous m'apportez là; s avez l'air tout fier de votre trouvaille. Comit appelez-vous cette plante? - Coqueret. - A i peut-elle être utile? - Je n'en sais rien. là justement ce que je veux que vous sachiez. ' vous a souvent servi de jouet; peut-être même z-vous goûté de ses fruits, malgré qu'on vous lit, - ce qui est une erreur, - qu'ils 'étaient 
vénéneux. Mais vous n'avez pas demandé si elle éta bonne à quelque chose : ce que nous allons voir.

Le Coqueret, ou Coquerelle, nom qui vient cette légère enveloppe rouge, s'appelle en botan que Alkékenge (Physalis alkekengi) : c'est v mot un peu dur; mais que voulez-vous? il vies de l'arabe! La plante est vivace; elle atteint 40 à 50 centimètres de hautour : on la rencont. fréquemment dans les champs et dans les vigne Sa famille est celle des Solanées à laquelle appa tient la Pomme de terre, dont les fleurs ressen blent beaucoup à celles du Coqueret. La partie plus remarquable, celle qui la rend familière à tor les enfants, sous lo nom de Pommes d'amour de Cerises d'hiver, c'est le fruit, qui, pendant l'a tomne, devient rouge et ressemble assez à une c rise. Ces fruits sont comestibles, d'un goût anal gue à celui de la tomate crue; cuits dans du siro ils constituent une bonne confiture. Remarqu comment ils se trouvent enveloppés par cette ca sule légère et demi-transparente, dont les nervur forment une élégante mosaiqque. Cette capsule n'e autre chose que le calice, qui, après la chute de fleur, a continué de croître, rapproché et soudé s découpures, de manière à mettro le fruit à l'al des intempéries.

Les baies fraîches, à la dose de quinze ou vingt sont diurétiques; l'infusion prolongée de baies s ches a la même propriété. Les feuilles peuvent se vir, comme celles de la Pomme de terre, à prépar des cataplasmes émollients; on a proposé les feu les, les tiges et les capsules comme fébrifuges. est certain qu'elles pourraient être utiles, si l'on pouvait se procurer rien de mieux; mais nous ves 
ns que nous avons autour de nous plusieurs mèdes de cette classe d'une action plus énerque.

Tous nos pourvoyeurs sont revenus les mains eines : je n'ai que l'embarras du choix. Prenons abord cette branche de Petit Houx (Ruscus acuatus), autrement dit : Houx-Frelon, Fragon, Fran piquant, Myrte sauvage ou épineux, Housson, use, Buis piquant. La médecine n'emploie que la cine blanche et un peu amère de cet arbuste, à quelle on a reconnu des propriétés apéritives et urétiques. On en prépare d'ordinaire une décocn dans laquelle on met 60 ou 80 grammes de cine fendue par litre d'eau.

Lo Petit Houx est de la famillo des Asparagies, dont le typo le plus vulgaire est l'Asperge, pour ne pas démentir leur origine, les jeunes usses du Petit Houx sont comestibles.

Le port de la plante est celui du Myrte, d'où lui nnent plusieurs de ses noms vulgaires. Ce que h prend généralement pour ses feuilles ne sont tre chose que des rameaux qui, au lieu d'être ids, s'élargissent et se terminent en pointe aiguë. vous allez facilement vous en convaincre. Rerquez où sont fixées les petites baies rouges qui - succédé aux fleurs; elles se trouvent, sur la ptendue feuille, à côté d'une petite écaille. Eh n, cette écaille, c'est la vraie feuille attachée au neau ainsi que le fruit. Nous avons donc ici, au a d'une monstruosité, une preuve nouvelle de simplicité avec laquelle la nature produit ses vres, même lorsqu'elle les revêt des apparences plus contrastées. 
Cet échantillon est un rameau de Passerage ( $L$ pidium latifolium) ou Passerage à grandes feu les, grande Passerage, de la famille des Cru fères. Le Chou, le Radis, le Cresson, le Raifort, Colza, la Giroflée sont aussi des crucifères. Dc cette famille, les fleurs ont quatre pétales ré liers, unguiculés, c'est-à-dire attachés au récepta par un prolongement mince et étroit du pétale. y a six étamines. L'ovaire très-allongé se tral forme, en mûrissant, en une gaîne formée de de valves qui s'ouvrent pour laisser échapper les gl nes fixées sur un châssis membraneux. Ces sor de fruits s'appellent siliques.

La Passerage est vivace, amie des terrains 0 bragés et humides. Elle atteint près de 1 mètre 50 hauteur. De sa tige robuste et rameuse partent grandes feuilles d'un vert bleuâtre, ovales-lancéolé Les fleurs se montrent en juin-août, elles sont ble ches, très-petites, groupées en grappes serrées qui f ment une panicule, nom que l'on donne à une esp d'épi droit formé de la réunion de petites grapp

Toute la plante, mais principalement les feuill offre une saveur poivrée, âcre. Les feuilles cc tuses, appliquées sur la peau, la font rapidem, rougir. Cette action rubéfiante a été utilisée con les douleurs névralgiques et rhumatismales. I feuilles séchées n'ont plus de vertu, mais on p récolter en automne la racine pour s'en servir toute saison à l'intérieur. On ne sait pas encore b régler l'emploi de la Persicaire, par conséquen1 mieux est de s'abstenir. Pour ce qui est de guéri rage, comme l'indique son nom, cette plante n'a une vertu plus spécifique que tant d'autres succ sivement préconisées et abandonnées par la méı 


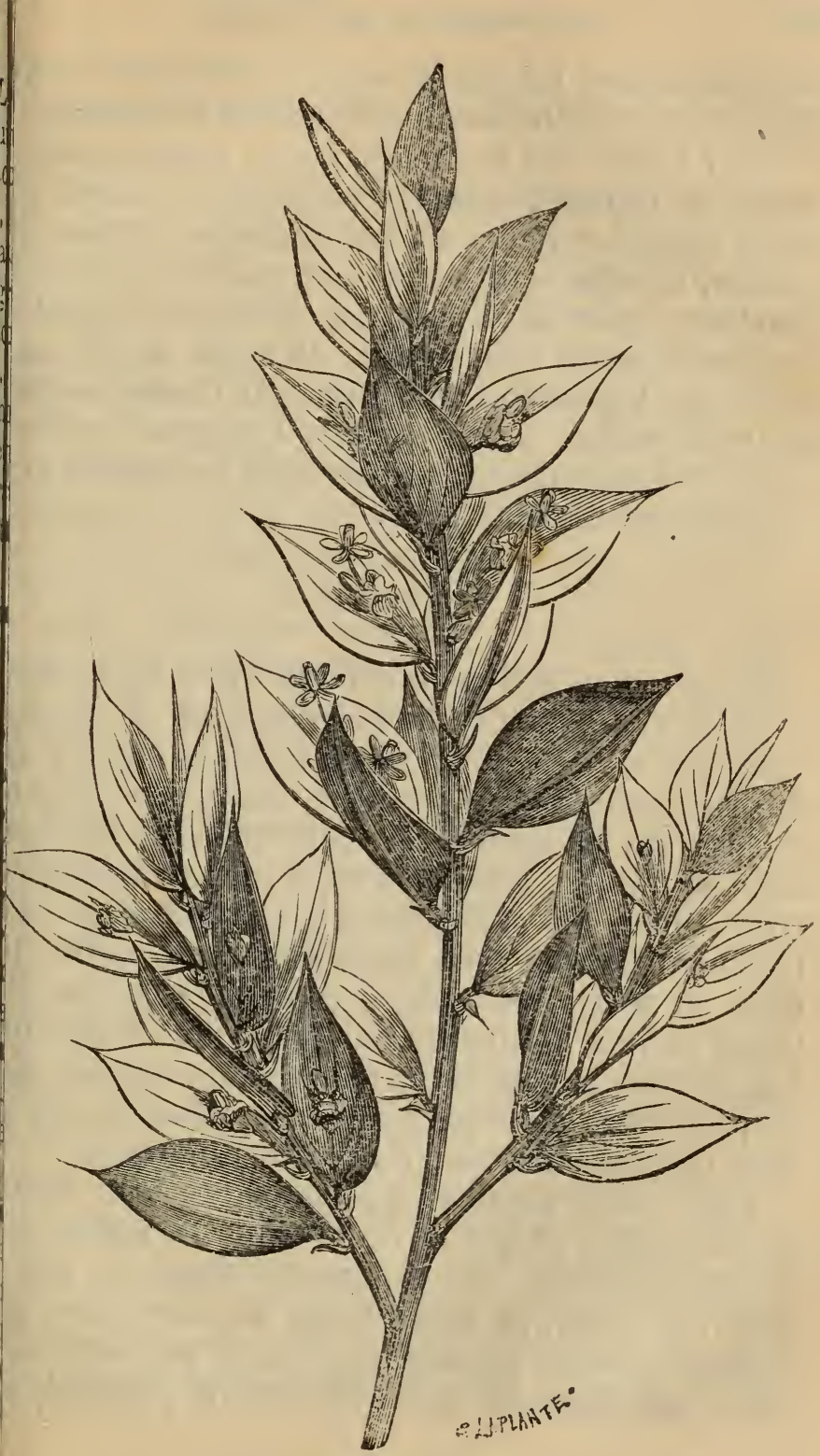

Branche de Petit Houx. ! 
cine populaire. La cautérisation profonde de la ple doit, dans tous les cas, précéder les essais curati

Pour éviter une confusion facile, notons qu'il y aussi la Petite-Passerage, Lepidium iberis, c croît dans les lieux arides, dont les feuilles sc petites, étroites, qui porte une panicule de flet écartées; puis la Passerage sauvage, Lepidiu ruderale, amie des ruines, et enfin la Passere cultivée, Lepidium sativum, ou Cresson alén dont les feuilles remplacent le Cresson. Toutes trois sont stimulantes, utiles dans le scorbut et maladies scrofuleuses.

Nous avons fait aujourd'hui une ample récc dans la campagne et dans les jardins de quelqu amis que nous avons visités. J'espère que la qu lité va répondre à la quantité. Asseyons-nous b à l'aise sur le gazon, et mettons un peu d'or dans ce fouillis. Cherchez vous-mêmes à réunir Graminées, les Solanées, les Ombellifères et : tres familles que vous savez déjà reconnaitre. $P$ dant ce temps, et tout en répondant à vos qu tions, je vais mettre de côté quelques échantill que je vous ai fait choisir à dessein, comme do de propriétés analogues sur lesquelles je me p pose d'appeler aujourd'hui votre attention.

Voici sept plantes appartenant à des fami très-diverses, et qui rentrent dans la même cle de médicaments : les purgatifs. Avant do les é dier en détail, je suis obligé de faire une pe incursion dans le domaine de la médecine, p vous expliquer ce que c'est qu'un purgatif, d 
les circonstances on en doit faire usage, quelles rences on observe dans l'effet des remèdes de 3 catégorie.

doit être bien entendu entre nous, en principe, le médecin seul est réellement compétent pour rescription des remèdes, et que l'on doit coner d'avoir recours à ses soins dès le commenent d'une maladie même peu dangereuse en prence. Mais en pratique il arrive que les habis des campagnes ne recourent à l'homme de qu'à la dernière extrémité. Le malade est imiatement assailli par des officieux, des guériss, voire même des demi-sorciers, vantant à mieux mieux leurs recettes infaillibles. Il est ; utile, nécessaire, de faire contre-poids à ces Lences en vulgarisant les notions les plus élétaires de la médecine. Il arrivera infailliblet que, dans le plus petit village, quelques peres déjà un peu instruites prendront goût à e étude et acquerront, sur les vertus et l'emploi plantes, des connaissances qui les mettront à he de rendre de grands services. Leurs bons es étant désintéressés, et leurs conseils appuyés des notions saines, bien qu'incomplètes, elles arderont pas à remplacer les guérisseurs à hoires et les personnes d'ailleurs dévouées qui raient pas les mêmes titres à la confiance. Plus l'exercico de la médecine étant devenu une tion publique, chaque village aura son docteur on officier de santé, les humbles volontaires de de guérir abdiquant toute initiative, seront ; ses ordres des auxiliaires précieux. Puisse ce ps être proche! En attendant, faisons de notre ux pour que les souffrants, les pauvres, les dé- 
laissés soient secourus dans les meilleures ec tions possibles.

Revenons aux purgatifs. Ce sont des méi ments qui augmentent et altèrent d'une mas sensible, mais passagère, les évacuations int nales. Ceux qui purgent doucement, sans ir tion, sont appelés laxatifs; on donne spécialer le nom de purgatifs à ceux dont l'effet est marqué; enfin, les drastiques sont ceux dont tion est très-forte et même violente. Comme purgatifs sont presque toujours des remèdes goût ou d'une odeur désagréable, on chercl masquer ce défaut par le sucre et des substa odorantes, ou bien on les administre sous form pilules. Quelques-uns sont très-bien absorbés lavement; d'autres sont assez énergiques if qu'on puisse les employer en frictions sur la p'

Après avoir pris un purgatif, on éprouve du laise, le pouls s'accélère, on ressent une cont tion pénible des intestins; la sécrétion de la 1 du suc pancréatique et du suc intestinal est $n$ blement augmentée aux dépens de la partie liq du sang, d'où vient la soif qui accompagne résultats de cette médication. Il y a donc d effets bien marquants : augmentation des foncti vitales de l'intestin et des organes qui y dévers leurs sécrétions; diminution de la masse du sa qui équivaut dans presque tous les cas à une saigh

Le besoin d'un purgatif est généralement il qué par l'aspect blanchâtre de la langue, le dégi les nausées, le manque d'appétit. Si l'on donne remède dans l'espoir de soulager ou de guérir hydropisie, on se gardera de satisfaire ensuit soif du malade, car il agit alors en augmentant 


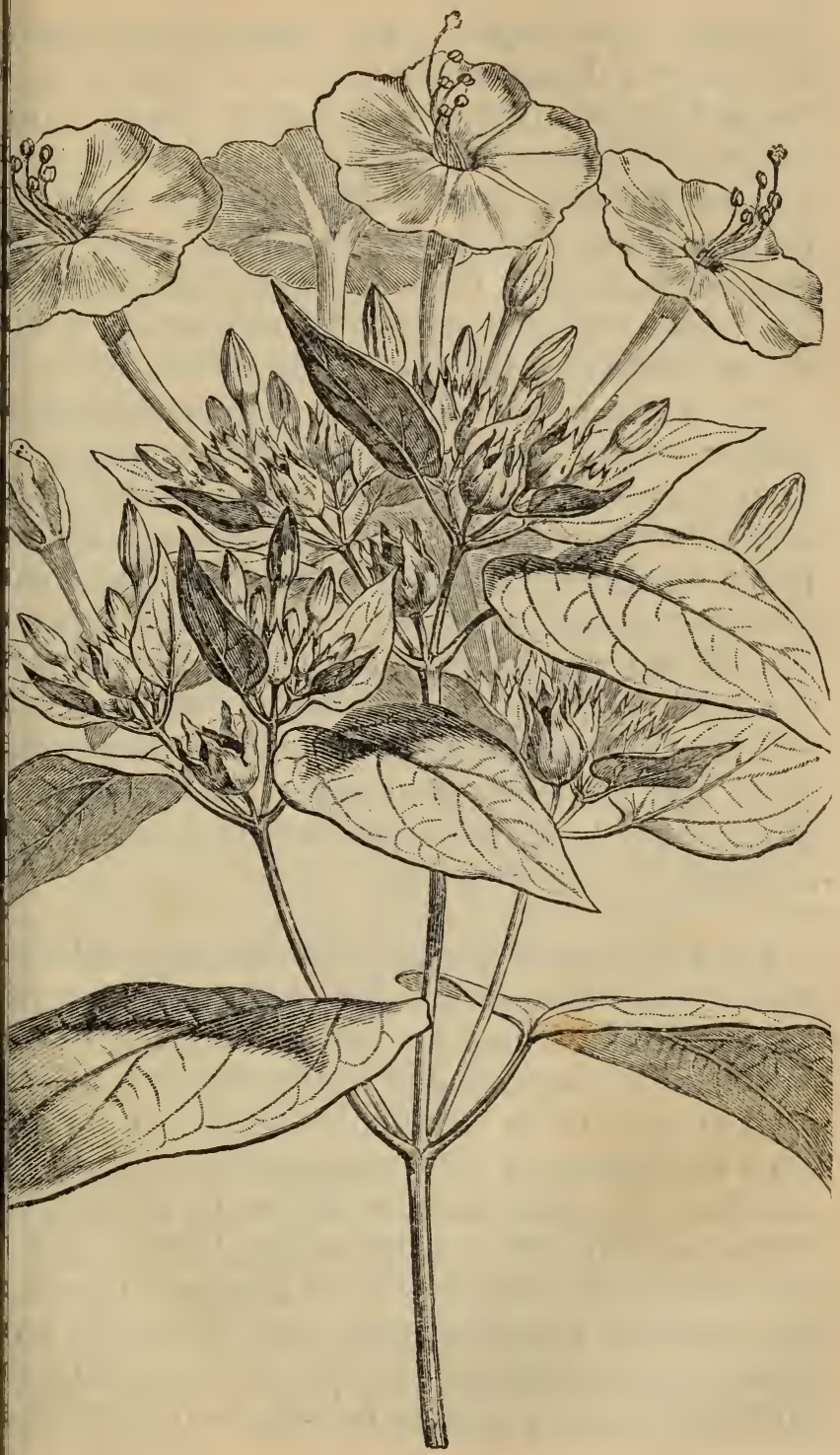

Sommité de Belle de nuit. 
fonctions d'absorption, par suite d'une élimin notable de liquides formés aux dépens du \& Dans les cas oú il y a irritation, fièvre, il fat contraire réparer les pertes par des boissons é lientes, bouillon aux herbes, bouillon léger, $t$ de chiendent, etc. On évitera de se purger pa temps très-chauds ou très-froids, lorsqu'il n'y pas urgence; et, le jour où l'on aura pris méde on se préservera du froid et de l'humidité.

On a beaucoup abusé des purgatifs, puis réaction étant venue, on les a trop négligés. Ad nistrés à propos, ils peuvent faire avorter un $g$ nombre de maladies; pour beaucoup ils co tuent un remède assuré. Nous trouverons sou dans nos promenades des plantes purgatives à degrés, au moins aussi efficaces que les rem exotiques les plus renommés, et pour lesquelle n'aurait pas assez d'éloges si elles venaient de Nous qui ne cherchons dans la médecine n mode, ni le luxe, nous étudierons leurs propril avec un soin tout spécial.

Vous avez sans doute remarqué souvent dans jardins ces belles fleurs dont la forme campanu c'est-à-dire en forme de cloche, rappelle le Lise des champs. Ce sont des Belles-de-Nuit (Miral jalapa), type de la famille des Nyctaginées, pla dont les fleurs ne s'ouvrent que le soir. Il y a variétés rouges, blanches, jaunes et panachées. Belle-de-Nuit est originaire du Pérou; mais s'est très-bien acclimatée en Europe. On la si sur couche, au printemps; on la plante, en 1 dans une terre légère et riche, et l'on jouit, de juillet jusqu'aux gelées, de ses fleurs nombret 
sées en bouquets au sommet des tiges, qui nnent de 50 à 60 centimètres. On peut aussi erver les racines dans un lieu tempéré pendant r, et les replanter au printemps. Cette plante pu chez nous plusieurs noms vulgaires : on elle Jalap à fleurs pourpres, faux Jalap, Jalap ène, Nyctage du Pérou. La partie active est cine en forme de fuseau, noirâtre en dehors, the en dedans, d'une odeur nauséeuse, et saveur âcre.

ssédant la Belle-de-Nuit, nous n'avons nullebesoin du Jalap du Pérou; notre Jalap indiest tout aussi bon, et ne coûte rien. On peut hinistrer en poudre, à la dose de 2 à 4 gramdans un verre d'eau miellée; il convient même enfants, chez qui il tue et fait évacuer les vers tinaux. La décoction de 4 à 8 grammes de e concassée, dans 150 grammes de bouillon de ou de poulet, produit un effet un peu moins que la poudre, et sera donnée de préférence personnes délicates. On a obtenu de bons réts de l'emploi prolongé de la Belle-de-Nuit dans cas d'hydropisie simple, de rhumatisme chroe, de maladies rebelles de la peau. En somme, une belle et bonne plante, à laquelle nous erons une place d'honneur dans notre herbier.

us rappelez-vous avolr vu s'ouvrir en décemdans les plates-bandes couvertes de neige, la ière des fleurs, la Rose de Noël? Sa corolle antée de givre semble vouloir nous dire que ature est encore active pendant son sommeil; nble plante s'épanouit en dépit des frimas, me l'espoir survit aux épreuves. Entre les 
grandes feuilles coriaces, d'un vert triste, de lées, profondément divisées et ouvertes comme main, on voit apparaître sur une hampe nue un ton d'un blanc rougeâtre, d'abord courbé vers le mais qui se dresse peu à peu et déploie cinq pé rosés. Un faisceau d'étamines jaunes occupe le ce!

Les botanistes l'appellent Ellébore NOIR ( $H$ borus niger). Elle est de la famille des Renonc cées, dont nous aurons occasion d'étudier plusi individus très-importants : la Renoncule, l'Acr le Pied d'alouette, la Clématite, la Pivoine, I colie. Toutes ces plantes sont très-actives, et 1 qu'on les emploie avec intelligence, elles peur rendre de grands services.

La partie la plus usitée de l'Ellébore noir es

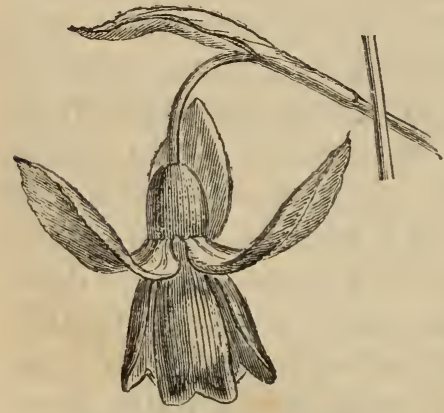

Fleur a silucivore blane racine. C'est une sou noirâtre d'oủ partent fibres épaisses. On la cueille à l'automne, ol sèche rapidement, et la conserve dans un $v$ bouché. Elle perd as vite ses propriétés, et ] doit l'employer fraî autant que possible.

La Rose de Noël c: spontanément dans montagnes d'Italie, de la Suisse et du midi de France, mais on la cultive partout en Europe. aime un terrain sec, léger, une exposition à soleil. On la multiplie généralement par éclats la souche. Les graines, semées aussitôt après l, maturité, donnent des variétés à fleurs plus gri des ou plus colorées. 
anciens regardaient l'Ellébore comme le ur remède contre la folie, mais la plante

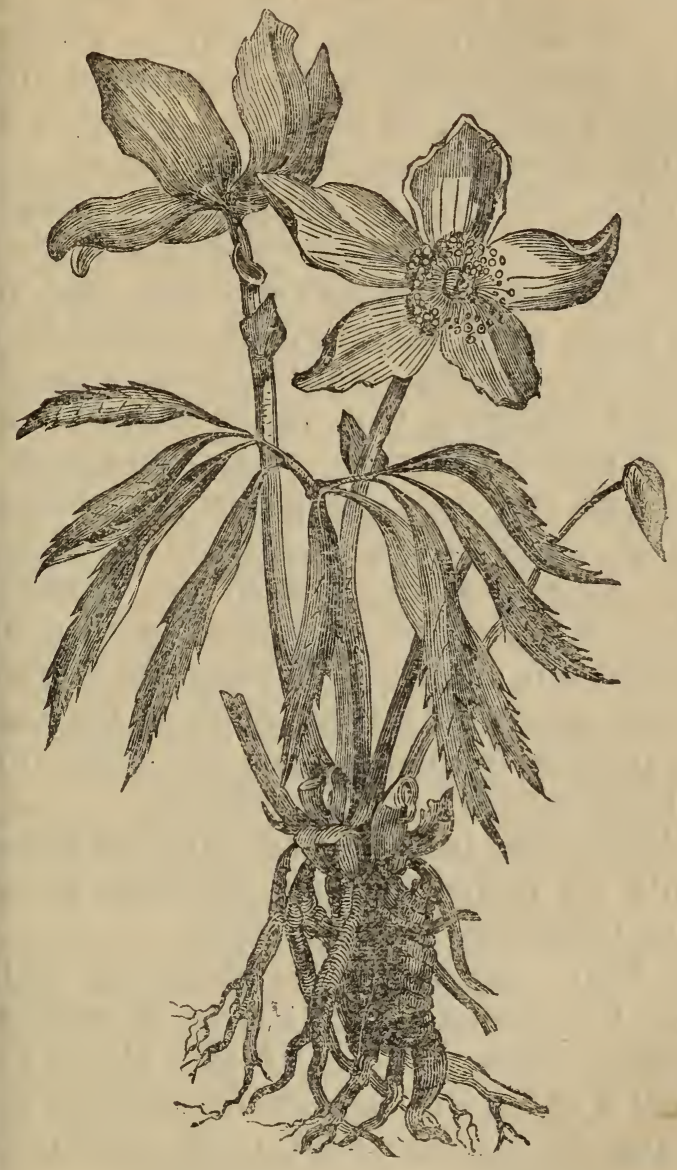

Ellébore noir ou Rose de Noël.

employaient diffère un peu de la nôtre. Cent les aliénistes utilisent encore aujourd'hui 
les vertus altérantes et l'action dérivative puiss de l'Ellébore noir dans les affections mentales ne sont pas accompagnées de fièvre, d'inflan n tion ou de désorganisation des organes vitaux,

L'Ellébore purge à la dose de 1 gramme à 1. 50 centigrammes de poudre, administrés dan, l'eau miellée, du vin, etc. On prépare l'infusio la décoction dans la proportion de 1 à 5 gran par litre d'eau, que l'on fait prendre par ver mais on peut, sans inconvénient, mettre la $\mathrm{m}$ dose dans une quantité moitié moindre de liqi

C'est une substance trop active pour l'empl comme purgatif ordinaire, même chez des s robustes, et l'on no peut guère conseiller faire usage autrement que sous la direction médecin. Elle produit souvent de très-heu. effets dans la paralysie, la goutte, le rhumati les maladies chroniques de la peau. De nombre expériences prouvent son utilité dans les cas o. dropisie passive fréquents chez les personnes habitent des contrées marécageuses.

Les vétérinaires introduisent dans les sétons fragments de racine de Rose de Noël, pour duire une inflammation artificielle.

Il faut bien vous garder de confondre l'Ellé noir avec l'ElLÉBore BLANG (Veratum album), pelé aussi Varaire, Vraire, Vérâtre blanc. Cel appartient à la famille des Colchicacées. C'est plante que le médecin seul peut employer danger.

J'ai mis ici à part trois échantillons d'arbri d'abrisseaux qui vous sont familiers : lo Fusai Bourdaine et le Nerprun. Tous trois appartien 
famille des Rhamnées, et jouissent, à divers rés, de propriétés semblables.

renons d'abord le Fusain (Evonymus europæus). $t$, vous le voyez, un arbrisseau très-rameux, que rencontre partout dans les haies et les bois. l'appelle Bonnet de prêtre et Bonnet carré à e de la forme de ses fruits divisés en trois ou angles, et qui, dans le mois de septembre, nent, en mûrissant, une belle couleur rouge. rameaux sont toujours opposés, les feuilles s, ovales, lancéolées, bordées de fines dents. fleurs, qui se montrent en mai-juin, sont es, à quatre pétales oblongs, blanchâtres et osées en corymbe, c'est-à-dire en parasol à ns inégaux. Le Fusain se multiplie de semis e rejetons et on le cultive comme plante d'anent; tous les terrains lui conviennent. s feuilles, l'écorce et les fruits du Fusain lent une odeur désagréable, nauséeuse. Toutes barties contiennent un principe irritant, âcre. produit, chez l'homme et les animaux, des voements et des selles accompagnées de coliques. jeunes pousses sont un poison mortel pour les tons, les chèvres et même les vaches. Les fruits, dose de trois ou quatre, purgent fortement et doit en adoucir l'action par des tisanes émoles de mauve, de graines de lin, ou par du llon de veau. Le mieux est de ne pas s'en servir l'avis du médecin. Nous avons bien assez de purgatifs innocents pour ne pas recourir à dont l'emploi peut offrir quelque danger. Mais xtérieur, la décoction d'une once de fruits bien 3, dans un litre d'eau, aiguisée d'un peu de gre, est un remède populaire justement ap- 
précié contre la gale. Elle peut aussi être uti pour animer et améliorer les ulcères indolents. If poudre des semences fait mourir les parasites la tête. Enfin, les vétérinaires guérissent la gale autres affections parasitaires des animaux av une décoction de feuilles, de fruits et d'écorce das du vinaigre. C'est donc une plante à la fois dang reuse et utile, qu'il vous importe de connaître.

Le Fusain est employé dans l'industrie. En cr. cinant avec soin dans des tubes de fer les rameai fendus en trois ou quatre brins, on obtient 1 crayons dits fusains, dont la trace s'enlève facil ment, ce qui les fait adopter pour tracer $d$ esquisses. Les artistes s'en servent aussi pour fai rapidement de grands croquis. Le bois de Fusai calciné en vase clos, est un de ceux que l'on pr fère pour la confection de la poudre.

Passons à la Bourdaine ou Bourgène (Rhamnil. frangula), appelée aussi Nerprun-Bourdaine, Aul noir et Bois noir. Elle crolt dans les halliers, 1 taillis, surtout dans les terrains frais et ombri gés. On la reconnaît facilement à ses tiges trè rameuses, sans épines, couvertes d'une écor noirâtre tachetée de blanc. En août-septembre montrent de petites fleurs jaunes ou verdâtre axillaires, c'est-à-dire partant de l'aisselle des feu les, et auxquelles succèdent de petites baies $q$ deviennent noirâtres en mûrissant. Ces fruits co tiennent trois noyaux cartilagineux, coriaces.

La partie la plus usitée est l'écorce moyen: ou seconde écorce, c'est-à-dire celle qui se trou' entre l'écorce et l'aubier. On la récolte un $p$ après la floraison. Elle peut se conserver assez lon 
2ps. Cette écorce est jaune, d'une odeur nauséaide et d'une saveur amère. On peut l'employer is la teinture en jaune, ainsi que les baies, mais leur préfère avec raison les fruits d'une variété amune dans le midi de la France et que l'on d sous le nom de Graines d'Avignon. 'écorce de Bourdaine fraîche, surtout celle de la ne, est trop active, elle provoque des vomisseits en même temps que des selles douloureuses. s après sa dessiccation, elle constitue un purf dont l'action se rapproche de celle de la Rhupe; on n'a plus à craindre ses effets drastiques. fusion ou la décoction de 20 à 40 grammes de e écorce dans un demi-litre d'eau corrstitue un purgatif ordinaire qu'il est facile de se procurer out sans frais. On peut aussi administrer de - à trois grammes de poudre dans des confis ou du miel. Elle convient très-bien aux en, si l'on a soin de proportionner la dose à leur car elle tue et fait évacuer les vers intestinaux. s fruits passent pour purgatifs, mais c'est une ir. On peut en manger un grand nombre sans re incommodé.

e forte décoction de racine de Bourdaine dans au et du vinaigre s'emploie à l'extérieur dans êmes circonstances que celle du Fusain. trefois l'administration de la guerre avait le de mettre la Bourdaine en réquisition pour la ation de la poudre, mais maintenant on l'ade gré à gré. Son bois blanc, fibreux et le sert à faire des allumettes, des chapeaux, nniers et autres ouvrages.

pi maintenant le Nerprun (Rhamnus cathar- 
ticus), vulgairement : Bourg-épine, Nerprun pus gatif, Nerprun, Epine de cerf.

Comme ses deux congénères, c'est un arbrisseai très-rustique, qui s'accommode de tous les sols de toutes les expositions, bien qu'il préfère un te rain frais. Ses rameaux épineux le rendent trè propre à former des haies impénétrables. Ses fleu petites, d'un blanc verdâtre, réunies en bouquets l'aisselle des feuilles, fleurissent en avril-mai ; ell produisent des baies de la grosseur d'un pois, vert d'abord, et qui deviennent noires à la fin d'octobs époque à laquelle on doit les recueillir.

Une de ces baies bien mûres, écrasée entre doigts, donne un suc visqueux d'un rouge noirâtr qui devient promptement d'un vert sombre contact de l'air. Les fruits de Nerprun du col merce sont souvent mélangés de petites prunel et de fruits de Troëne. Voici le moyen de les connaître. La prunelle renferme un seul noyau; baie de Troëne en contient deux; tandis qu'il y a quatre dans celle du Nerprun.

A ce propos, laissez-moi vous donner un b conseil. Lorsque vous cueillerez ou ferez cuei pour la vente quelque remède indigène, attack le plus grand soin à ce qu'il remplisse toutes conditions que l'on est en droit d'attendre, et tout, ne vous laissez jamais tenter par la mauva pensée d'augmenter votre gain par la fraude. penseriez-vous de l'herboriste ou du pharmac qui, en vous payant, mêlerait à des pièces d'arg quelques pièces de cuivre argenté, pour dimin sa dépense? Eh bien! en falsifiant un remède l'addition de produits analogues en apparen non-seulement vous donnez du cuivre en p 
argent, mais vous exposez la vie de vos semables! Le marchand vous volerait; vous, vous lez et vous tuez! Et cependant ces choses-là so ht tous les jours. Dans le commerce des drogues, est habitué à ne plus ajouter foi qu'aux résulis de l'analyse et du plus minutieux examen. A science de la fraude il a fallu opposer une autre ence qui en déjoue les piéges et les méfaits. La ix de votre conscience suffira pour vous empêor de commettre une fraude qui peut avoir des nséquences funestes ou du moins constituer un ritable vol. Mais votre intérêt même vous y enge. Quand un marchand s'est laissé tromper une s, sa juste méfiance lui fait souvent une règle de plus rien recevoir de l'individu qui l'a trompé, souvent même il l'étend à tout un canton. Dans pays qui produisent la Salsepareille, le Rahia, l'Indigo, le Quinquina, on a vu des proces entières obligées de renoncer à une industrie rative parce que des spéculateurs malhonnêtes, isant de la confiance de leurs correspondants, ient envoyé quelques chargements adultérés. s innocents ont souffert pour les coupables, on plus voulu entendre parler du produit de ces ions. Croyez bien que les choses se passent par(t ainsi sur une échelle plus ou moins vaste, et p si l'on veut faire revivre chez nous l'usage et conséquent le commerce des simples, la prere condition est d'y apporter le soin le plus upuleux et la plus rigide honnêteté. ues baies de Nerprun fraîches, écrasées, sont jères, âcres, nauséeuses. Elles constituent un gatif énergique, drastique et anthelmintique, n effet sûr, et qui n'offre pas d'inconvénients 
pour les tempéraments robustes. Voici les diffé. rentes formes sous lesquelles on peut l'adminis. trer : en décoction, 20 à 30 baies par litre d'eau suc exprimé : 8 à 30 grammes, édulcoré avec di sucre, du miel ou de la mélasse : sirop composé de parties égales de suc et de sucre, de trente à cen grammes. Enfin, on peut avaler tout simplement comme des pilules, de dix à vingt baies, fraîches or sèches, en ayant soin de boire, à de courts inter valles, un mucilage de graines de lin, du bouillos de veau, ou quclque autre tisane émolliente, afi de prévenir les coliques. Les graines légèremen torréfiées et pulvérisées purgent bien à la dose d'en viron 4 grammes en pilules ou dans du miel, dr sirop. On peut aussi en faire une décostion à 1 dose de 8 grammes dans une ou deux tasses d'eav

On trouve dans l'écorce moyenne du Nerprun le propriétés éméto-cathartiques du Fusain et de 1 Bourdaine, mais il vaut mieux s'en tenir à l'usag de ses fruits.

Nous allons maintenant nous occuper d'une plant fort intéressante, le Ricis (Ricinus vulgaris), d. la famille des Euphorbiacées. Toutes les plante de cette famille sont douées de propriétés très-ac tives. La plupart renferment un suc laiteux plu ou moins caustique ou un principe résineux âcr Elles agissent à l'extérieur comme rubéfiantes; l'intérieur, comme émétiques et cathartiques. Que. ques-unes constituent de véritables poisons. A c groupe appartiennent le Réveil-matin, d'un usag populairo contre les verrues et les cors; l'Epurg dont les semences sont purgatives et les feuille vésicantes 
Le Ricin est un arbre originaire des contré chaudes de l'Afrique et de l'Inde, où il s'élève ju qu'à douze à quinze mètres. Dans les pays temp rés où il s'acclimate facilement, ce n'est plus qu'u plante annuelle à croissance très-rapide, et qui s'élève pas au delà de trois mètres. On la culti dans les jardins à une exposition chaude. Dans n départements du midi et particulièrement aux en rons de Nîmes, il est assez répandu pour fourr presque entièrement à la consommation de France. En serre, on peut le faire durer deux trois ans. C'est une des plus belles plantes de n jardins, et l'on devrait la trouver partout où l' cultive un coin de terre. On le sème au printem dans un sol léger, substantiel et frais. Sa cultt tend à prendre beaucoup d'accroissement dept l'introduction en Europe d'une espèce de ver-à-st qui se nourrit de ses feuilles.

La tige droite, rameuse, fistuleuse, prend $u^{*}$ teinte rougeâtre d'un bel effet, tandis que les jeur pousses sont d'un vert glauque. Les feuilles, $p$ tées par de longs pétioles, sont divisées en sept neuf lobes inégaux, pointus et dentelés en scie. I fleurs qui paraissent en juillet-août, forment if thyrses ou épis élégants à l'extrémité des ramear Le même épi contient des fleurs mâles à la base, des fleurs femelles au sommet. Les fleurs mâles c un petit calice à cinq divisions qui disparaît sc, une multitude d'étamines. A la fleur femelle sil cède, peu de temps après la chute de la fleur, vit capsule à trois côtes saillantes, couverte d'épir molles, comme le marron d'Inde. Cette capsi contient trois graines ovales de la grosseur d' petit haricot, tachetées de rouge sur fond grisât] 
r saveur, d'abord oléagineuse et douceâtre, det bientót nauséeuse, âcre et brûlante. On en - par la pression, après les avoir broyées, ou f'ébullition dans l'eau, une huile incolore, visise, d'une odeur et d'une saveur peu prononcées hd elle est fraîche, mais qui rancit assez vite. huile de ricin se prescrit dans tous les cas où désire simplement obtenir un effet laxatif. Elle ient très-bien dans les cas de constipation opie. Elle ne fatigue pas les personnes qui soufd'hémorroildes; on peut sans crainte l'admier aux enfants. La dose varie de 15 à $60 \mathrm{gr}$. un adulte, mais rarement on a besoin de déer $30 \mathrm{gr}$. On la dissimule dans du bouillon aux es chargé d'oseille, ou du thé additionné de le citron. Mélangée avec de la poudre de sucre lu miel, elle ne répugne plus aux enfants. Il se garder d'avaler deux ou trois graines en$\mathrm{s}$, comme on le fait quelquefois pour produire purgation énergique; il pourrait en résulter accidents graves. Mais l'émulsion formée en it de 20 à 50 centigrammes de semences avec aune d'œuf et du sucre, ou bien avec de l'eau mée, constitue un purgatif très-énergique et e action sûre. Toutefois, comme il pourrait uire la vomissement chez des personnes déli, on fera bien de la prendre en deux ou trois à une demi-heure d'intervalle. C'est surtout ue d'avoir toujours une petite provision de ies, pour les employer sous cette forme, que erais à voir quelques pieds de Ricin dans tous ardins. Peu de plantes réunissent à un plus degré l'utile à l'agréable. 
Voyez maintenant cette grosse racine et ce meau chargé de graines que nous avons cueil] même temps. Qui d'entre vous peut me dire nom? - C'est la Vigne du diable. - C'est le N du diable. - La Couleuvrée. - En effet, \} quelques-uns des noms qu'on lui donne, mais en a bien d'autres. On l'appelle Vigne blan Racine vierge, Colubrine, Feu ardent : toute: son vrai nom est Bryone (Bryonia alba). C'est Cucurbitacée, comme la Citrouille.

La Bryone est vivace, on la rencontre fréqu ment dans les haies, où ses tiges minces, gr pantes, longues de 3 à 4 mètres, s'accrochen moyen de longues vrilles. Comme la Citrouille, porte des fleurs, mâles et femelles, petites, c blanc terne, disposées en bouquets peu touff l'aisselle des feuilles. Aux fleurs femelles, qui se tinguent par un pédoncule ou support très-co succèdent des baies grosses comme un pois, acquièrent en mûrissant la belle couleur rouge vous voyez. On croit à tort qu'elles sont vé neuses. Les graines qu'elles renferment contienr une forte proportion d'huile. Le principe actif ré: dans la racine, ordinairement grosse comme bras, pivotante, charnue, jaunâtre à l'extéri blanche au dedans, marquée transversalement plusieurs sillons. Cette racine étant vivace, on $\mathrm{f}$ se la procurer fraîche toute l'année. Afin de l'ar sous la main pendant l'hiver, le mieux est di récolter à l'automne et de la conserver dans sable comme les racines des Légumineuses. Pou dessécher, on la coupe par rouelles que l'on en en laissant un peu d'espace entre chacune, et on l' pose à la chaleur modéréo d'un four. Dans cet é 
conserve bien ses propriétés médicinales. Elle rès-amère, d'une saveur nauséabonde, et cède ement à l'eau ses principes actifs.

tre !es mains d'un médecin expérimenté la ne, selon les doses et le mode d'emploi, agit ne vomitive, purgative, résolutive et rubée, ce qui la rend utile dans les hydropisies, la lysie, le rhumatisme, les affections catarrhales, yssenterie. Malheureusement ce n'est pas un de à la mode, et peu de praticiens savent en parti. Dans la médecine usuelle, nous ne dela considérer que comme un purgatif capable emplacer le Jalap. Sèche et pulvérisée, on en e de deux à trois grammes. Le suc à la dose à 12 grammes dans du bouillon, produit toude bons effets, ainsi que la décoction de 10 à rammes par litre d'eau. On l'administre aux aux avec non moins de succès. La dose, pour beuf, est de deux à trois onces en décoction. pliquée à l'extérieur, la Bryone rend de grands ces. La racine fraîche pilée et cuite avec du oux forme une excellente pommade contre la Il suffit de cinq ou six frictions à un ou deux d'intervalle. La pulpe de racine fraîche exerce fiction excitante et résolutive sur les engorges indolents des articulations et sur les tumeurs uleuses. Comme rubéfiante et par conséquent ative, elle est apte à soulager les douleurs uses et rhumatismales.

ilà des titres suffisants pour faire placer la ne au rang des plantes les plus utiles. Mais ce pas tout. Non-seulement elle se prête très-bien ie la Clématite et la Vigne vierge à garnir les lles et les treilles, mais elle se contente de 
toutes les expositions et des terrains même les p ingrats. Vivace et agreste, sa racine acquiert s vent la grosseur de la cuisse. Si on la râpe dans l'eau et que l'on enlève par des lavages les par fibreuses légères et les principes âcres qui en $f$ un agent curatif, on obtient pour résidu une féc aussi blanche et aussi saine que celle de la Pom de terre. La pulpe simplement lavée à grande e et soumise aux procédés ordinaires de fermentati donne un alcool de bonne qualité. Enfin, ses gr nes nombreuses sont très-riches en huile, et $m$ teraient d'être récoltées pour en extraire ce prod Il est donc à souhaiter que l'on multiplie la Bryc par semis ou par éclats de racines, dans les terre improductifs, à la lisière des bois, au pied des hi et le long des routes. Dans les cantons où la ms d'œuvre est à bon marché et les ressources abondantes, cette plante, trop longtemps négli fournirait à l'industrie agricole une véritable chesse.

En attendant que nous arrivions en pleine c: pagne, je vais, mes amis, vous dire quelques $n$ d'une plante qui vous est très-familière, mais $d$ vous ignorez les propriétés : c'est le Buis. Il a : pendant quelque temps, en Allemagne, d'une gra réputation, mais sous un nom supposé. Un hon possédait un remède secret pour la guérison fièvres, et l'on citait par centaines ses cures autl tiques. Le bruit en vint jusqu'à la cour, et Josep acheta le secret de ce remède au prix de 1500 rins. Son but était de vulgariser, en la publi 
découverte si utile. Mais quand on sut que le eux remède était simplement lo Buis vulgaire jardins, le prestige disparut. Comment une se si commune pouvait-elle être bonne? On ne donna même plus la peine de l'essayer, la plante, t appréciée lorsqu'elle était inconnue, tomba s l'oubli lorsqu'on apprit son nom, et depuis, lques amis des simples, exempts de préjugés, seuls conservé la tradition de ses vertus.

e Burs (Buxus semper virens) est de la famille Euphorbiacées, dont nous avons déjà étudié individu : le Ricin. C'est un arbrisseau qui croît ntanément en Espagne, en Italie et en Suisse. is n'avons en France que deux forêts de Buis : e dans le Mâconnais, l'autre au mont Jura. Le is nain ou d'Artois, que l'on emploie en bordures is les jardins, se rencontre à l'état sauvage en sse et en Franche-Comté.

porte des fleurs des deux sexes, petites, partant f'aisselle des feuilles. Le fruit est une capsule montée de trois petites cornes, correspondant à s loges contenant chacune deux graines. On t en faire des semis en terre légère ou le multiir par marcottes. On greffe quelques variétés, Ime le Buis panaché.

es feuilles de Buis ont une odeur vireuse un aromatique. On les mêle souvent, par fraude, elles du Séné. Elles sont purgatives à la dose deux à trois onces en infusion ou en décoction hs un litre d'eau, et de 3 ou 4 grammes en idre, sous forme de pilules ou mêlées à du miel. on action dans les fièvres intermittentes n'est moins réelle aujourd'hui qu'au temps de eph II. La meilleure manière de l'administrer 
est de donner de 2 à 3 grammes de feuilles en po dre, dans de l'eau sucrée ou tout autre véhicu au commencement de l'accès. On constate dès premières doses un changement notable, et l' obtient au bout de quelques jours, une guéris complète.

Le bois de Buis râpé, et surtout celui de la racir à la dose de 30 à 60 grammes en décoction dans litre d'eau, est un bon sudorifique, dont les effi sont en tout comparables à ceux du Gayac, dans affections rhumatismales chroniques et les accider qui en sont la suite, principalement les engorg ments d'articulations.

On emploie quelquefois les feuilles de Buis po. augmenter l'amertume de la bière et diminuer dépense de houblon. On reconnaît la falsificatior l'apparence un peu trouble du liquide et à sa save nauséeuse différente de l'amertume franche de bonne bière.

Lo Buis fournit le bois le plus dur et le pl compacte de nos climats. Sa densité égale ou su passe celle de l'eau. On l'emploie, comme vo savez, à une foule d'usages. On en fait des peign des boîtes, des toupies. C'est sur du buis que l' exécute les gravures dites sur bois.

Voici une bonne fortune. Cette belle plante vace, haute de plus d'un mètre, à tige simple droite, d'où partent des feuilles opposées d'un bl vert, ovales, aiguës, à nervures longitudinales sa lantes, c'est la Gentiane (Gentiana lutea), autı ment dite Grande-Gentiane, Gentiane jaune. Elle donné son nom à la famille des Gentianées do nous possédons en France plusieurs individus $\mathrm{i}$ 
téressants, entre autres, la Petite-Centaurée, d nous parlerons plus tard. Les plantes de cette mille sont remarquables par la présence d't amertume franche doueje de propriétés toniques ts prononcées, propriétés qui ont été reconnues d les pays les plus éloignés et utilisées d'une faço peu près uniforme.

La Grande-Gentiane est commune dans les partements du milieu et du midi de la France. peut la multiplier de semis ou d'œilletons, au pi temps, dans une terre fraîche, à mi-ombre. F sieurs variétés, entre autres la bleue, sont cu. vées dans les jardins pour la beauté de leurs fle disposées en faisceaux à l'aisselle des dernit feuilles, et qui s'ouvrent en juin-juillet.

La partie active est la racine pivotante, sp. gieuse, rugueuse et ridée, qui atteint quelquefoi grosseur du poignet. On la récolte au plus tôt fin de la deuxième année, après la chute des feuil Sa saveur est d'une amertume franche intense, âcreté.

Vous entendrez dire que la Gentiane est v dans la dyspepsie, les flatuosités, la diarrhée, scrofules, la jaunisse, le scorbut, la goutte, fièvres intermittentes. Cela est très-vrai. Mais l' tichaut, le Houx, la Chausse-trappe, le Saule seront pas moins efficaces. Est-ce donc par un tagonisme spécial que ces plantes agissent ces maladies? Evidemment non. Leur effet siste simplement à produire dans les organes modification qui les rend capables de résist l'influence de la maladie et de rejeter, s'il y a I les principes étrangers dont la présence troul leurs fonctions. Le remède a donné du ton, $d$ 
e aux nerfs, aux muscles, aux viscères: les hts eapables de produire cet effet s'appellent toues. La Gentiane en est un type parfait; elle it que par un principe amer pur. D autres, me la Camomille, l'Absinthe, sont à la fois toes et excitants, parce que au principe amer ils nent un aromate. Il y a des plantes qui exerune action tonique d'une espèce particulière, spécialement à la présence d'un acide ou le plus ent à celle du tannin, comme dans l'écorce de e : ce sont des toniques astringents. A l'exté, ils resserrent les fibres et peuvent ainsi s'opaux hémorrhagies et aux écoulements mors provenant d'un relâchement des tissus; ils iennent ou diminuent l'inflammation en s'opht à l'afflux du sang. A l'intérieur, ils agissent ne anti-putrides, et une fois mêlés au sang, le ont plus coagulable, ce qui explique leur utilans les hémorrhagies et les flux de toute naLes toniques sont naturellement contre-inlés dans les maladies inflammatoires. Ces ipes bien simples étant posés, je vais tâcher, notre promenade, de vous montrer le plus d nombre possible de plantes appartenant à classe de remèdes, pour laquelle nous n'avons ument rien à envier à l'étranger.

is revenons d'abord à notre Gentiane. Perne contestait ses vertus fébrifuges lorsque le quina était inconnu en Europe. Depuis, on les es absolument. Il n'en est pas moins vrai que entiane, administrée en poudre dans du vin, * l'accès, soit seule, soit unie à l'écorce de e ou d'Aune, ce qui vaut encore mieux, guérit ritement les fièvres de printemps et d'automne, 
et même les fièvres paludéennes. Elle offre de plv l'avantage de prévenir ou de guérir également le engorgements de la rate ou du foie, et l'état mi ladif qui persistent trop souvent après ces affer tions. Aucun tonique n'est plus efficace dans 1 . maladies scrofuleuses sauf peut-être le Noyer, ma dans ces cas, il est indispensable de placer le $\mathrm{m}$ lade dans des conditions hygiéniques convenable bonne nourriture, bon air et beaucoup de soleil.

La décoction de Gentiane concassée à la do de 30 grammes par litre d'eau, et prise à la do de 60 à 100 grammes par jour, continuée penda longtemps, est le meilleur tonique dans toutes ! affections produites par un manque de vitalit Le vin de Gentiane préparé en faisant macér 30 grammes de racine coupée dans 500 grammes vin, et pris par petits verres avant le repas, très-efficace contre la dyspepsie; les digestions viennent plus faciles et la santé revient bientôt.

Maintenant nous allons nous reposer un peu, je vais vous donner, au sujet des feuilles, quelqu connaissances botaniques que nous mettrons so vent à profit.

Vous avez tous remarqué des différences bi notables dans la forme, la couleur et la dispositi des feuilles des végétaux. Nous avons vu celles la Capucine s'étaler en disque, celles du Genévr s'allonger en aiguilles, celles de la Rose de Noël séparer comme les doigts d'une main. Nous alli examiner aujourd'hui leurs caractères princips et nous familiariser avec les noms qui servent à qu désigner.

On distingue dans la feuille le limbe, partie 
ppée, et le pétiole ou support. Quelquefois lo ole manque et la feuille est dite sessible. Celle part directement de la souche ou racine s'apo radicale.

ne feuille est simple, si elle n'a qu'un limbe; e, lorsque le limbe est divisé en plusieurs pors obtuses; et pennée ou composée, si plusieurs ts limbes qui prennent le nom de folioles vienH s'attacher au pétiole par des pétiolules, me dans cette feuille de faux Acacia. Lorsqué tiole cummun se ramifie et porte d'autres pés sur lesquels s'attachent des folioles, la feuille pelle décomposée.

feuille du Ricin est fide, c'est-à-dire fendue lusieurs parties ; quand les fentes arrivent jusu pétiole, comme dans le Chanvre, la feuille est partite, ou palmée, comme dans le Marron-

aminons maintenant les nervures qui forment me le squelette des feuilles. Dans le plus grand bre, on voit une nervure principale qui va de se au sommet, et donne naissance à d'autres ures latérales disposées à intervalles à peu près $\mathrm{x}$ : le Laurier nous en offre un exemple. Soucomme dans la Mauve, un certain nombre de ures principales, partant du pétiole, rayonnent les contours du limbe. On voit aussi des ners parallèles se diriger de la base du limbe au net comme dans la Gentiane, l'Orchis. est un autre point qu'il nous importe de reuer. Les feuilles ne sont pas disposées de la - manière sur la tige. On les dit opposées si sont placées en face l'une de l'autre à la même ur ; alternes, si lo point d'insertion est diffé- 


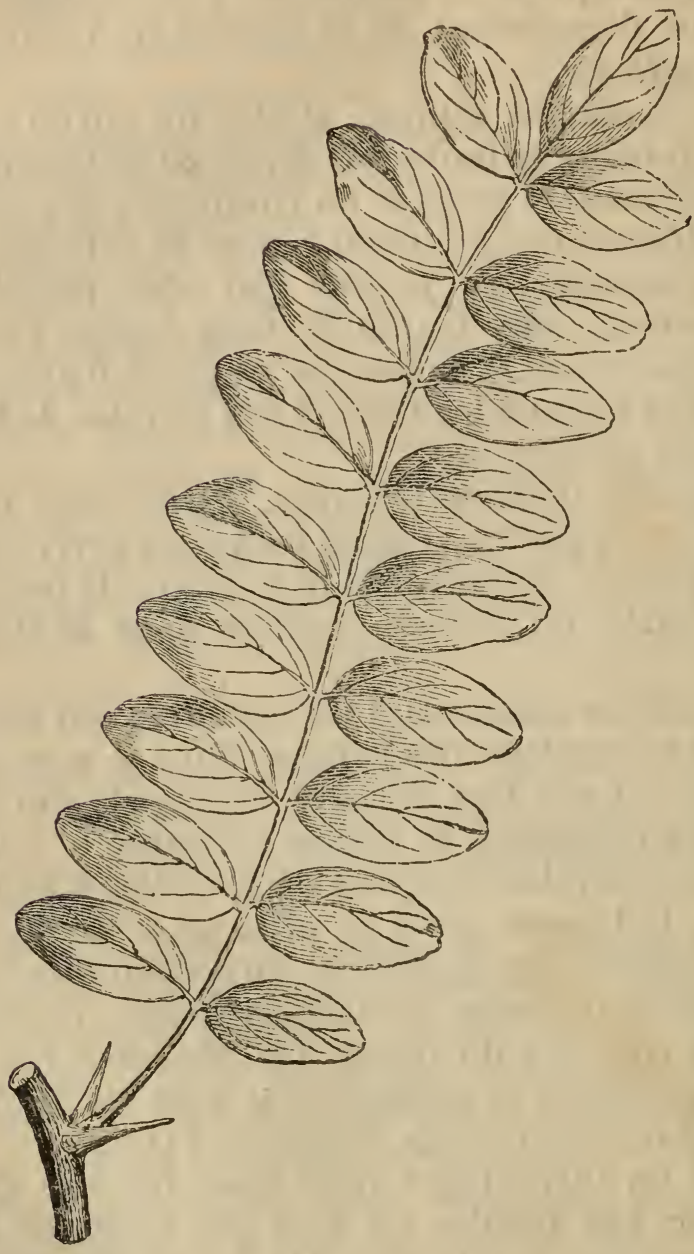

Fenille composée.

pées en touffes autour de la tige, comme dans Thym, le Genévrier. 
e pétiole des feuilles est souvent accompagné, base, d'une espèce de petite feuille que l'on cime stipule, dont la forme est très-variable. lquefois, comme dans l'Eglantier, il fait corps - le pétiole.

e même que la peau de l'homme et des ani$x$ est criblée de pores, ouvertures microscopiqui donnent passage à des liquides et à des l'épiderme des feuilles est percé d'un grand bre de stomates, orifices par lesquels s'évapore 've et qui servent également à l'absorption et au gement de gaz nécessaire à la vie de la plante. urais bien d'autres choses intéressantes à vous au sujet des feuilles, mais je veux me borner notions les plus indispensables, sauf à entrer quelques détails, à mesure que l'occasion s'en entera. Ne vous effrayez pas des mots noux. Limbe, pétiole, verticille, ne sont pas plus iles à retenir que substantif, adverbe, interon, que l'usage vous a rendus familiers. Toute uestion est de n'en pas apprendre beaucoup à is et de les entendre répéter souvent, ce que ne manquerons pas de faire dans nos promeds.

plante vivace dont vous me voyez extraire la e racine pivotante, fibreuse, brune en dehors, jaune en dedans, c'est la Patience (Rumex ntia), de la famille des Polygonacées. Cette farenferme un assez grand nombre de végétaux cés à feuilles alternes munies d'un stipule, et ivant leur développement, sont roulées en deNous lui devons la Persicaire, dont je vous ai tout à l'heure; la Bistorte, un astringent 
dont nous avons trouvé un exemplaire; le Blé no l'Oseille, la Rhubarbe.

Les tiges de Patience, vulgairement Parel Dogue, sont hautes d'environ 1 mètre 50 centin tres, droites, cannelées, un peu rameuses. I feuilles pétiolées, sont grandes, ovales à pointe, peu ondulées.

Elle développe en juin-août ses fleurs très-petit verdâtres, sans corolle, disposées en espèces d'é terminaux formés de nombreux verticilles. On cultive dans les jardins pour ses bourgeons et feuilles légèrement acides, que l'on apprête com celles de l'Oseille, et que l'on nomme Epinards i mortels. Il faut semer ses graines sur place à l' tomne à toute exposition, dans une terre fraîche substantielle.

Il y a plusieurs espèces de Patience, parmi 1 quelles la Patience sauvage ou Lampée (Rur. acutus); la Patience grépue (Rumex crispus) e Patience a feuilles obtuses (Rumex obtusus). I toutes ont les mêmes propriétés. Leur action tonique, dépurative et même purgative à doses peu élevées. On fait surtout usage de la racin décoction à la dose de 30 à 60 grammes par 1 d'eau. C'est un remède très-populaire dans campagnes, principalement contre les maladies la peau. Les feuilles contuses peuvent exercer heureuse influence sur les ulcères indolents $e$ engorgements lymphatiques. On prépare un onguent contre la gale en mélangeant parties ég d'axonge, de soufre et de pulpe de racine de tience ramollie en la faisant bouillir dans $d u$ naigre; mais il faut observer que le soufre contribuer pour une bonne part à la guérison. 


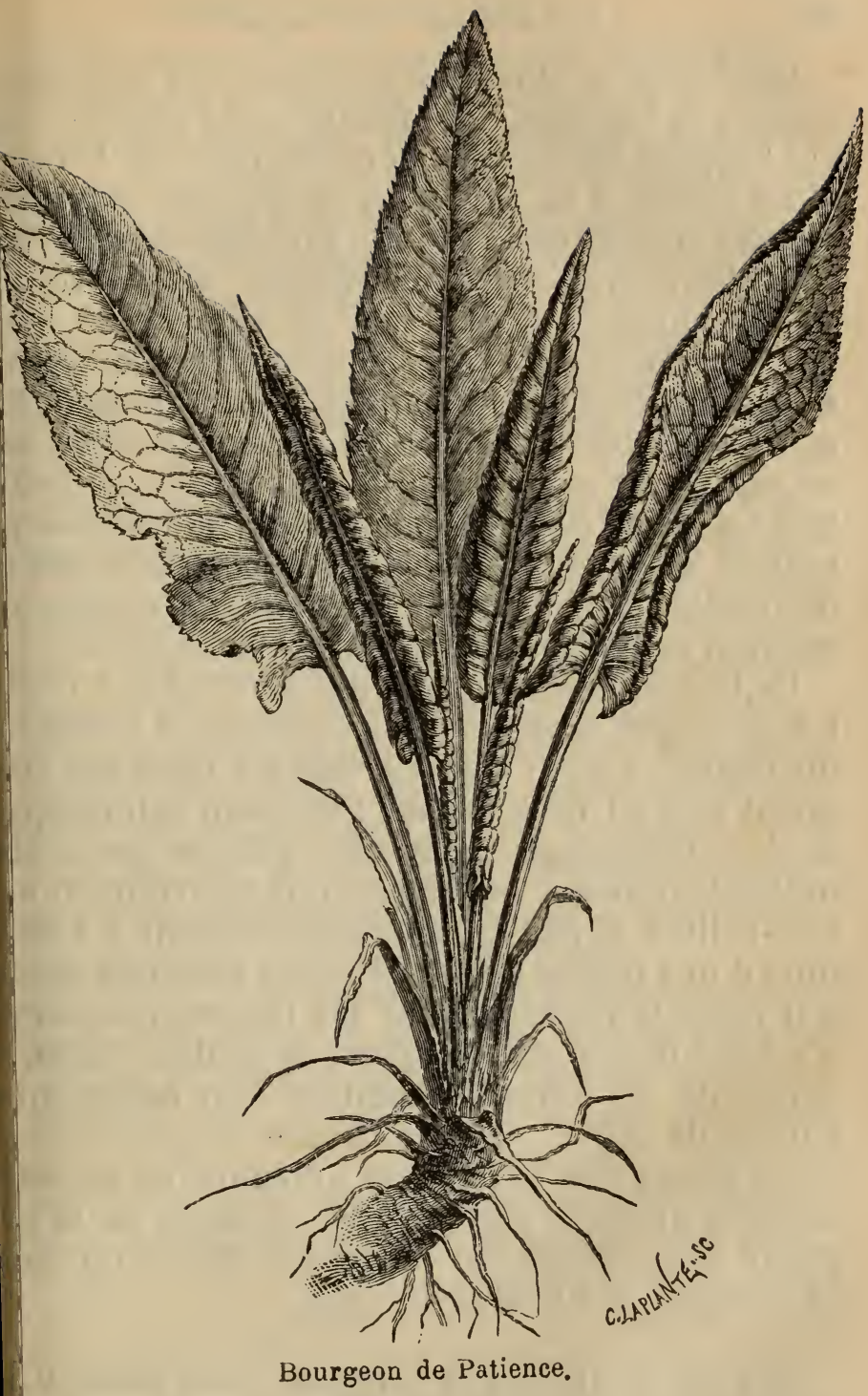


La Bistorte (Polygonum bistorta) appartient commo la Patience, à la famille des Polygonacées On l'appelle quelquefois Renouée-Bistorte. Un cou] d'œil donné à sa racine vous explique son nom elle est contournée deux fois sur elle-même. Brun en dehors, rougeâtre en dedans, elle porte de sillons annulaires, d'où naît un chevelu de nom breuses radicelles. Son principe actif est lo tannin si abondant qu'on l'a employée au tannage. Ell contient en outre beaucoup d'amidon que l'on pet en séparer et que l'on utilise en Russie en le mê lant au pain. Hẹureusement nous ne sommes pa réduits à cet expédient, et nous possédons, en ce de disette, bon nombro de plantes sauvages qr peuvent contribuer largement à l'alimentation.

La Bistorte est assez commune dans les prés les pâturages ombreux. Les animaux, à l'exceptio du cheval, aiment à en brouter les tiges qui atte gnent 30 à 50 centimètres. Elles sont cylindrique striées, fistuleuses, noueuses, garnies de feuill ovales-lancéolées. Les fleurs, qui s'ouvrent en ma très-petites et très-nombreuses, forment à l'extr mité d'une hampe grêle, un épi en forme de massi qui rappelle l'inflorescence du Roseau massette. n'y a pas de corolle; le calice, de couleur rosée, e surmonté de longues étamines qui dépassent surface de l'épi.

On peut semer la Bistorte à l'ombre, en automn aussitôt après la maturité des graines, ou la pr pager par division des pieds. La souche ou raci se récolte à la fin de novembre.

Je vous ai dit que je comptais vous parler d'u Polygonacéo que l'on appelle Persicaire (Polyg 
im hydropiper). C'est que je savais où en trouen abondance. Nous y voici. Elle aime, vous ez, les lieux humides, on la rencontre dans les (sés, les marais. Ses noms vulgaires sont nomux : Poivre d'eau, Piment d'eau, Renouée âcre, rage, Herbe Saint-Innocent. Si vous goûtez ses illes, qui ressemblent à celles du pêcher, et surit ses petites graines triangulaires, vous leur trouez une saveur poivrée, âcre et brûlante, qui honce, à n'en pas douter, des propriétés énerues.

Jous reconnaîtrez la Persicaire à sa tige nue, ueuse, un peu rougeàtre, garnie de quelques raaux, haute de 30 à 80 centimètres, portant des illes alternes lancéolées aiguës; mais principa-. hent à ses fleurs très-petites, formées simplement n calice rosé, six étamines et trois styles port chacun un stigmate. Ces petites fleurs sont posées en épi grêle, filiforme, arqué, garni de ites écailles et s'ouvrent de juin à octobre.

Dn emploie la plante entière, fraîche, car les unes augmentent son activité. Elle perd beauup de son énergie par la dessiccation.

1 l'intérieur, la Persicaire agit comme stimulant rgique; mais c'est une de ces plantes injusteint abandonnées des médecins, et dont les effets t été jusqu'ici mal étudiés. Le mieux est done ne pas l'employer à l'intérieur sans l'avis de omme de l'art, en attendant que des expériences n dirigées nous apprennent comment utiliser ses priétés.

Jes services à l'extérieur sont incontestables. Elle at remplacer la moutarde dans la préparation des apismes. Une forte décoction tue l'acarus de la 
gale. Le suc de la plante, pur ou étendu d'eau, c stitue l'un des remèdes les plus efficaces pour $r_{c}$ ver les ulcères atoniques, séparer les chairs mo: et hâter la cicatrisation. Il est bon, dans ce de mêler le suc à une décoction concentrée feuilles de noyer. Les vétérinaires connaissent bien cette propriété et se servent aussi de ci plasmes de Persicaire pour résoudre les engor ments lymphatiques des articulations. Sa décoc constitue en outre un bon gargarisme dans les d'angine, d'aphthes et d'ulcérations de la gorge, peut même calmer quelques maux de dents I veux.

Il ne faut pas confondre la plante qui nous occ avec la Persigaire douce (Polygonum persica et la Persicaine amphibie (Persicaria amphibiu qui se distinguent par leurs fleurs en épis oblor cylindriques, compactes, et dont les propriétés s encore mal définies.

Voyons si vous avez retenu ce que je vous ai au sujet des différentes espèces de feuilles. - C ment appelons-nous celle-ci ? - Feuille compo - Pourquoi? - Parce qu'elle est formée de pet feuilles ou folioles, fixées par des pétiolules, petits pétioles, à un pétiole commun qui leur de support. - Fort bien. Vous voyez que la Be nique n'est pas si difficile à apprendre.

Vous connaissez tous la petite plante que je vi d'arracher. Elle croît dans les lieux humides bien que les chevaux et les vaches la brouten que les oies la recherchent, elle fait quelquefois tort aux prairies où elle se multiplie trop. Les $p c$ sont très-friands de sa racine et bouleversent 
tre pour s'en emparer. On l'appelle vulgairement gremoine sauvage, Bec-d'oie, Potentile ansérine. in vrai nom est Argentine (Potentilla anserina) i à la couleur blanche soyeuse du duvet qui recuvre la partie inférieure des feuilles. Elle apparfint à la famille des Rosacées. La racine contient a tannin et de l'amidon. Quant à ses propriétés ( sont, à un degré un peu plus faible, celles que Hus avons reconnues dans la Bistorte de sorte que rus n'y reviendrons pas.

Pour terminer notre promenade, nous allons détcher de ce vieux mur une petite plante presque impante que vous avez vue partout sur les toits, Is décombres et dans les terrains arides. C'est la jRMiculaire (Sedum acre), d'une famille remaraable par l'épaisseur des feuilles et parfois des yes, celle des Crassulacées, qui comprend ce que in peut appeler les plantes grasses de notre cliat, entre autres la Grande Joubarbe. La Vermilaire n'a pas manqué de parrains. On la connaît us le nom de Petite Joubarbe, Sédon âcre, Orpin ûlant, Poivre des murailles, Pain d'oiseau, Herbe int-Jean.

Il suffit de l'avoir vue une fois pour la reconître à ses tiges longues de 5 à 10 centimètres, u rameuses, à demi rampantes, couvertes de uilles épaisses, ovoïdes, et à ses petites fleurs unes en épis courts formant des corymbes terinaux.

Il est à peu près inutile d'en faire une provision, or la dessiccation lui enlève ses propriétés. Heuusement on peut se la procurer fraîche sans diffialté. Elle est d'un goût piquant, âcre, qui fait 
présumer des éléments très-actifs. Il faut, en effe la manier avec beaucoup de prudence. Une oni du suc produit un effet cathartique violent. Mais doses modérées, entre les mains d'un médecin, el

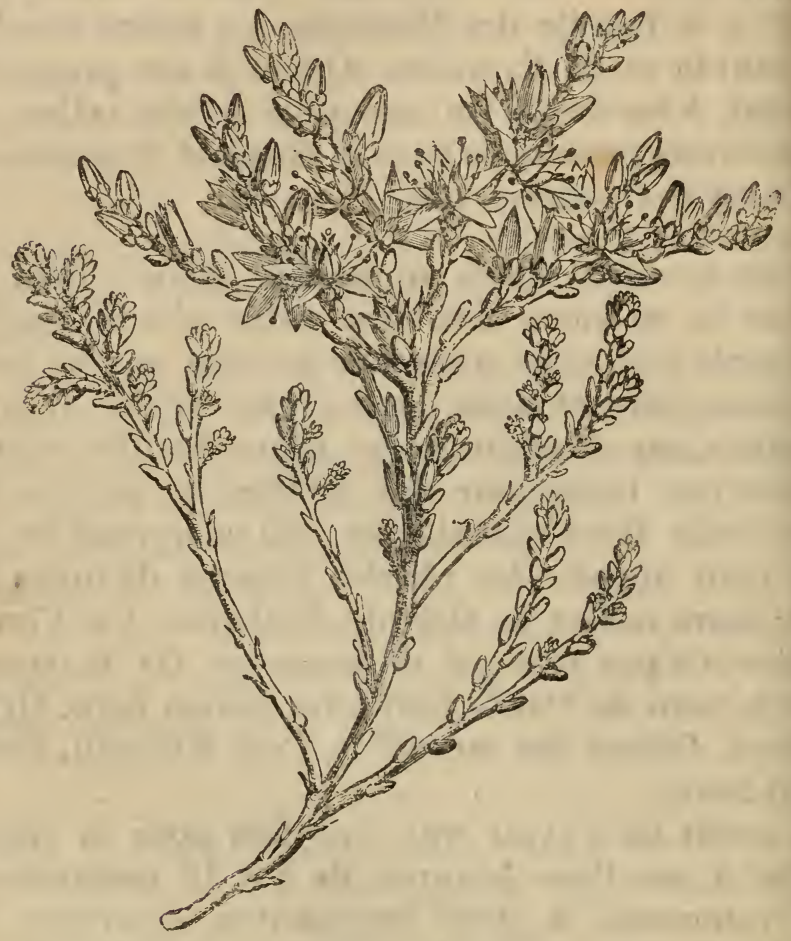

Rameau de Vermiculaire.

peut agir d'une façon remarquable comme remèd altérant, c'est-à-dire capable de modifier les fonc tions des organes, de les mettre à même de réagi contre la maladie. On a eu principalement à s'er louer dans le traitement du scorbut et do l'épi. lepsie. 
1 l'extérieur, on peut employer sans crainte le de Vermiculaire ou des cataplasmes faits avec plante pilée, pour provoquer la résorption des inchements, des engorgements glanduleux ou aphatiques, et surtout pour nettoyer et aviver vieux ulcères, même ceux qui affectent un catère cancéreux. En pareil cas, la Vermiculaire able agir avec plus d'énergie que la Persicaire t nous parlions tout à l'heure.

Ie passons pas devant ce buisson d'Epine-vinetTe s nous informer des propriétés de cet arbuste, la nature fait croître spontanément dans prestous les terrains, et que l'on cultive dans les cs et les jardins d'agrément. L'écorce de la tige jaunâtre; celle des rameaux grise, les feuilles tolées, obtuses, dentées en scie, sont disposées bouquets alternes. De fortes épines partent de la ssance des rameaux. Les fleurs paraissent en -juin. Elles sont d'un jaune pâle, disposées en ppes pendantes. Leurs étamines, au nombre de offrent un curieux exemple de la sensibilité des ates. Si vous en touchez une avec la pointe ne épingle, elle se contracte et va se poser sur istil.

'ous vous étonnez sans doute de m'entendre dire les plantes sont sensibles. Je ne prétends pas ; par là qu'elles sont susceptibles de douleur ou plaisir, mais seulement qu'elles sont capables, is certaines circonstances, d'accomplir des mounents provoqués par les agents extérieurs. Vous dez tous que les plantes dirigent leurs rameaux (s l'air et la lumière. Quelques-unes exécutent mouvements continuels. Si vous examinez 
attentivement une vrille de Bryone, vous la verr se dresser, se tordre et se contourner lentemen Vous avez entendu parler de la Sensitive, dont l

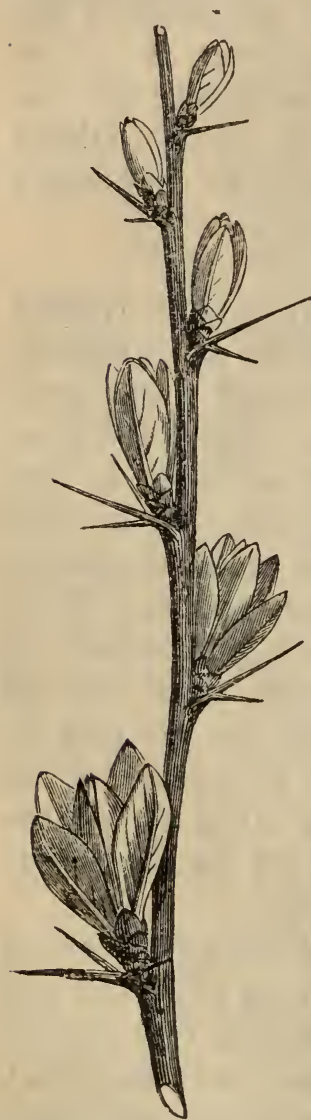

Bourgeond'Epine-vinette feuilles se ferment au moinds attouchement. Mais il $\mathrm{y}$ a ur plante bien plus curieuse encor c'est la Dionée attrape-mouche Ses feuilles, pórtées par un lon pétiole si large qu'on le prendra volontiers pour la feuille ell même, sont couvertes d'une l queur visqueuse qui attire li insectes. Mais si une moucl vient s'y poser, la feuille se repli les longs cils qui la bordent s'e tre-croisent, et le pauvre insec: demeure captif pour toujour car la feuille ne s'ouvre qu'apr sa mort, alors qu'elle n'est ply irritée par ses mouvements.

Maintenant que vous savez qu les plantes imitent quelquefo. les mouvements qui sembles n'appartenir qu'aux animaw vous n'oublierez pas, au pri temps, d'en faire l'expérience sı l'EpINE-vinetTe ou Vinettier $(\mathrm{Ba}$ beris vulgaris), appelée aus Berbéris, qui a donné ce nom la famille des Berbéridées, do] elle est le type.

Toutes les parties de la plante sont utiles : racine et les tiges sont employées pour teindre $\epsilon$ jaune la laine; le fil, lo coton et les cuirs; le si 
des baies donne avec l'alun une laque d'un 1 rouge; la seconde écorce de la tige, et surtout ' de la racine, est amère, tonique et légèrement gative; enfin ses baies rouges servent à prép une limonade préférable à celle de citron, très-1 dans les cas de fièvres inflammatoires, typho ou bilieuses. La décoction acidulee des feu forme aussi une bonne tisane rafraîchissante. E on prépare avec ses fruits écrasés, macérés । l'eau avec du sucre brut et quelques aromates, piquette agréable.

On récolte les fruits en automne, et on les sè ils conservent toutes leurs propriétés.

A côté de toutes ces qualités, l'Epine-vinet un défaut. Mais qui n'en a pas, même parmi plantes? L'important est de les connaitre. C'es fait avéré que la transformation d'un champig microscopique qui s'y attache fait naitre la ro: ou la carie sur le blé, le seigle et les autres céré qui croissent dans son voisinage. On fera donc de ne multiplier cet arbuste utile que loin des tures auxquelles il peut nuire. On peut en faire boutures, des marcottes, ou détacher des bi geons enracinés. Malgré ses épines, il ne fait de bonnes haies, parce qu'il se dégarnit du ba: ne souffre pas d'autres plantes près de ses raci qui épuisent le sol.

Voici nos pourvoyeurs qui reviennent les $\mathrm{m}$ pleines. Voyons un peu ce qu'il y a de bon c tout cela.

Pouvez-vous me dire le nom de cette brar 


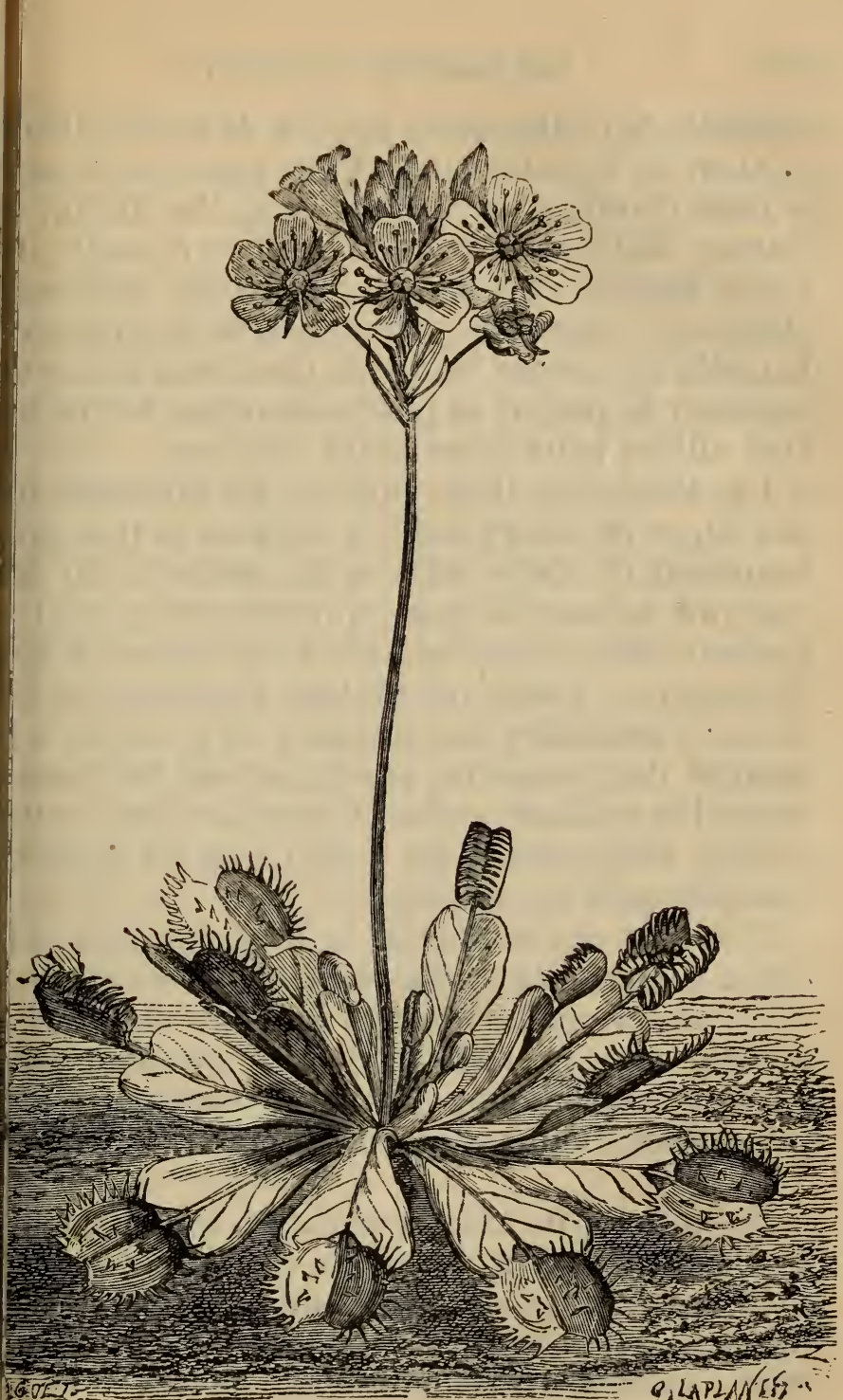

Dionée attrape-mouches.

je PAR'TIE. 
couverte de petites baies rouges, de forme allonge - C'est du Bois-Gentil. - Yous avez raison; mais a bien d'autres noms : on l'appelle Merlin, fa Garou, Malherbe, Tramentel, Bois d'oreille, Lo réole femelle. En botanique, c'est le Mézért (Daphne mezereum), famille des Daphnacées, laquelle appartient le Garou, dont nous allons avi occasion de parler, et plusieurs autres arbustes $q$ l'on cultive pour leurs fleurs précoces.

Le Mézéréon aime surtout les bois montue des Alpes et des Pyrénées; mais on le trouve da beaucoup de départements du centre et du nor c'est un arbuste élégant, qui atteint un mètre hauteur. Ses fleurs en forme de cloche, à qua découpures, roses, odorantes, disposées en fase cules, c'est-à-dire en faisceaux ou groupes, à l'e trémité des rameaux, paraissent en février-ma avant les feuilles. Celles-ci sont alternes, entièr ovales, lancéolées, d'un vert foncé en dessus, peu glauques en dessous.

Il ne faut pas confondre le Mézéréon avec la LA RÉole (Daphne laureola), dont les fleurs d'un jau verdâtre forment de petites grappes axillaires. $S$ feuilles sont persistantes; elles ne tombent $p$ en hiver. Quant aux fruits, ils sont noirs et $n$ pas rouges comme ceux du Mézéréon.

Enfin il y a un autre arbuste de la même famill le Garou (Daphne gnidium), ou Sain-Bois, q donne des fruits rouges, mais que l'on disting facilement des deux précédents par ses feuilles se siles, éparses, très-nombreuses, déliées à la bas étroites et lancéolées. Les fleurs, petites, odorante blanches ou un peu rougeâtres, sont disposées 
trémité des rameaux en panicule, grappe formée petits bouquets souțenus par un pédoncule comn. On trouve le Garou dans nos départements du di ; mais dans ceux du nord, où on le cultive, il a oin d'être rentré dans l'orangerie pendant l'hiver. outes les Daphnacées réclament une terre substielle et fraîche. On les sème en pots à l'automne is de la terre de bruyère, et l'on repique l'année ivante.

- Mézéréon, la Lauréole et le Garou ont des priétés semblables. Un fragment de leur écorce, jé sur la langue, produit bientôt une sensation lante, persistante, qui s'étend jusqu'à la gorge. feuilles fraîches et les fruits, frais ou conservés, sent à peu près les mêmes effets.

dose un peu forte, les Daphnacées sont des sons violents, et il n'appartient qu'au médecin s'en servir, à l'intérieur, comme dépuratifs, fonts et drastiques. Entre ses mains, elles peuvent ner des cures inattendues dans l'hydropisie, le matisme chronique, les scrofules, les tumeurs olentes, les engorgements et les affections darises. C'est un de ces agents énergiques, capables produire, par révulsion ou autrement, une perjation salutaire, une crise, qui permet aux anes d'éliminer les produits morbides, cause ou hltat de la maladie.

n comprenant ainsi la manière d'agir des remèon s'explique comment des plantes dont le hcipe actif est très-différent peuvent produire mêmes effets. Il n'y a pas de spécifique, c'estire de remède capable d'aller attaquer l'être ginaire nommé fièvre, rhumatisme, jaunisse, et le tuer en l'empoisonnant ou en se combinant 
avec lui de manière à former un nouvel être in fensif, de même que l'acide sulfurique, poison vi lent, en s'unissant à la chaux vive, autre poisc forme du sulfate de chaux, substance parfaiteme inerte. Il y a cependant des cas où les choses pe vent se passer ainsi, par exemple dans la gravel. mais ce sont des exceptions. En général, nous ig! rons quelle est l'essence des maladies, et nous d nons ce nom aux phénomènes, aux symptômes $p$ duits par la lutte du principe vital contre le troul survenu dans les fonctions ou dans la constituti des organes. Dans cette lutte, la nature est souve impuissante; les remèdes interviennent alors $p c$ exciter la réaction si elle est insuffisante, la moc rer si elle est trop vive. Tel est leur vrai rôle. ne guérissent point, ils dirigent simplement efforts de la nature. Envisagée à ce point de vi la médecine se trouve dégagée de toutes les supe titions, de tous les mystères, qui plaisent, il vrai, au grand nombre, toujours ami du merve leux, et dont savent tirer parti les charlatans ou habiles, mais qu'il importe de faire disparait pour le plus grand bien de ceux qui souffrent faut que la confiance du malade, cet auxilia puissant de la thérapeutique, au lieu de se fone sur les vertus occultes de quelque remède secr compte sur la sympathie et sur le savoir éclairé médecin.

J'espère que vous avez compris ma petite digr' sion; mais comme je tiens à vous bien inculq! ces idées, j'y reviendrai à l'occasion, lorsqu't plante me fournira une application remarqua des principes que je viens de vous énoncer.

Il est donc bien entendu que l'emploi des Dat 
cées à l'intérieur est réservé à des mains habiles expérimentées. A l'extérieur, leur omploi n'offro $s$ les mêmes dangers, et la médecine usuelle peut compter parmi ses agents les plus utiles. C'est zorce séchée que l'on emploie d'ordinaire. La is énergique est celle du Garou.

'écorce de Garou, ramollie par un séjour de elques heures dans de l'eau, et mieux dans du aigre, appliquée sur la peau et maintenue en ce par un bandage, produit au bout de vingtatre heures la rubéfaction, et au bout de quate-huit heures une vésication complète; elle a les cantharides l'avantage de ne pas irriter voies urinaires, de donner lieu à une exsudation liquide plus abondante et plus prolongée, et de pas laisser de traces. On lui reproche de causer fois une démangeaison pénible et une irritation parties environnantes; mais une décoction de feuil et de fleurs de Sureau suffit pour calmer petits accidents. Il sera donc bien d'employer le ou toutes les fois qu'il n'y aura pas besoin d'obeir une vésication rapide.

on nom de Bois d'oreille vient de l'usage que en faisait communément dans quelques cons du midi, avant qu'il fût connu des médecins. l'employait comme révulsif derrière les oreilles enfants, dans les engorgements des glandes du o, les maux d'yeux et les dentitions pénibles. Le xiou figure aujourd'hui dans les pharmacies; mais o bien d'autres plantes non moins utiles, dont les aitants de la campagne, sans autre guide que la ition, mettent chaque jour à profit les services, tindent laur place dins les livres classiques et as les officines! 
Passons à ce pied de Pariétaire (Parietaria off cinalis), que l'on a eu soin d'apporter tout entie: et qui fera un excellent échantillon. Vous diraitous ses noms vulgaires? Voici ceux que je cor nais : Perce-muraille, Casse-pierre, Herbe de Notr Dame, Espargoule, Vitriole, Panatage, Epinard ( muraille. Elle appartient à la famille des Urticée qui renferme, en outre de l'Ortie, d'où lui vies son nom, l'Orme, le Chanvre, le Houblon, Mûrier.

Elle croît sur les murs et les décombres. Si tiges atteignent de 50 à 60 centimètres; elles so; fragiles, rameuses, légèrement couvertes de poil garnies de feuilles, alternes, pétiolées, ovales-oblo. gues, velues et un peu rudes. Les fleurs, verdâtre très-petites, ont besoin d'être examinées à la louj pour bien reconnaître leurs caractères, qui mérite: de fixer notre attention.

Ces fleurs sont réunies à l'aisselle des feuilles, nombre de 3 ou 5 . Dans chaque groupe, il y a ur fleur bisexuée, c'est-à-dire contenant des étamin et un pistil, une fleur mâle, dans laquelle le pis ne s'est pas développé, et enfin une fleur femell qui n'a qu'un pistil sans étamines. Nous trouvo ici un nouvel exemple de l'irritabilité de certai: organes chez les végétaux. Si vous touchez u: étamine avec la pointe d'une aiguille, elle se $\mathrm{r}$ dresse comme un ressort qui se débande; l'a thère s'ouvre, et laisse échapper un nuage pollen.

La Pariétaire offre une saveur un peu salée, d à la présence d'une notable quantité de nitre (nitre de potasse) qu'elle contient, et auquel on doit att: buer ses propriétés rafraîchissantes et diurétiqu' 


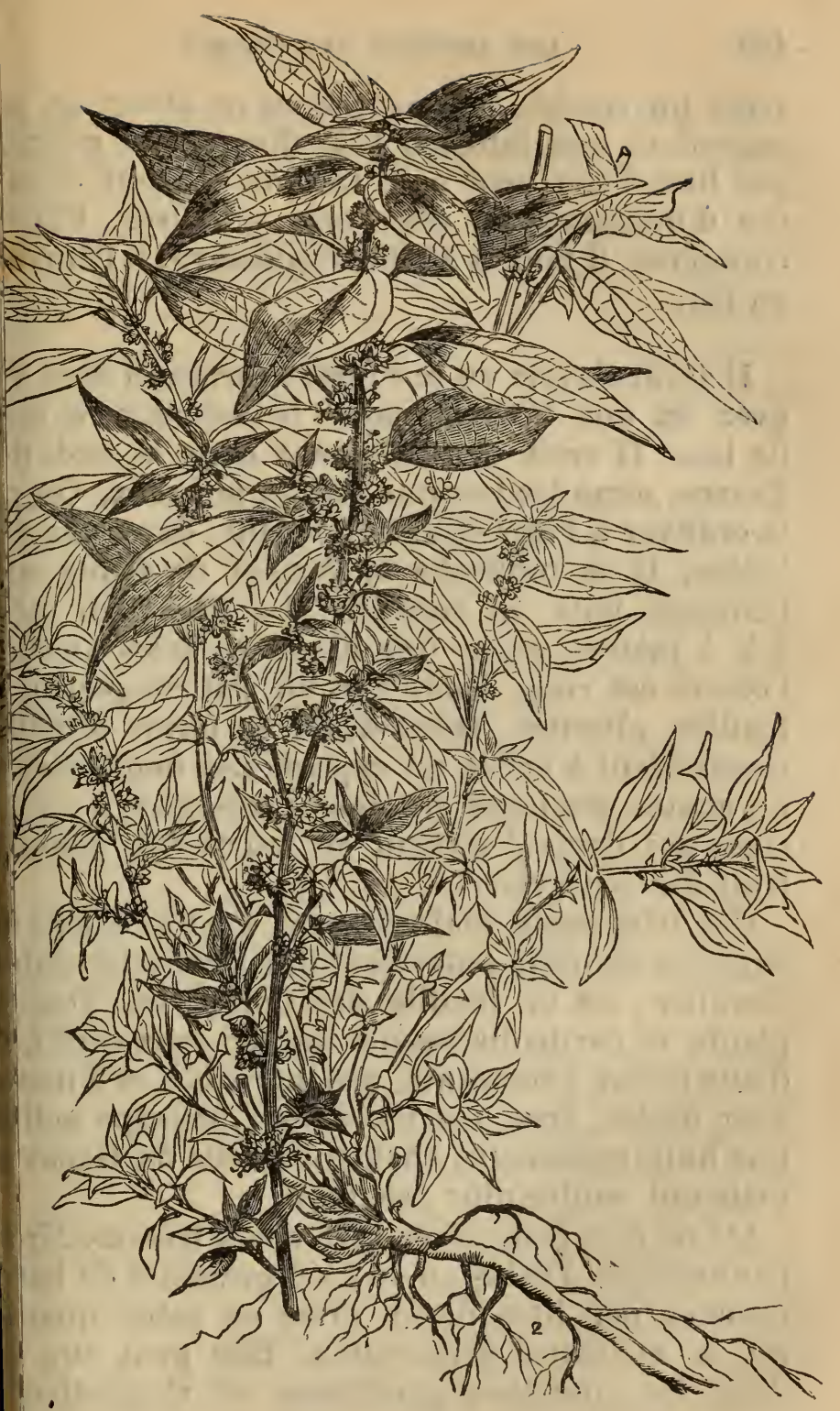

Pariétaire. 
C'est un remède populaire dont on abuse un pe cependant son infusion à la dose de 30 gramm par litre d'eau peut agir comme calmant dans cas d'inflammation des voies urinaires. Pour conserver, il faut la sécher rapidement à l'étuve au feu.

Il suffit d'avoir vu une fois l'arbrisseau dont vc avez ici une branche pour le reconnaitre mê: de loin. Il croît naturellement dans le midi de France, aime les terrains secs et pierreux. On pit le cultiver à l'exposition du levant, dans une te légère. Il se multiplie facilement de semis ou boutures faits en automne. La tige, qui attet 3 à 4 mètres, porte des branches très-ramifié l'écorce est rude, cendrée et un peu rougeâtre. feuilles, alternes, très-petites, en forme d'écail ressemblent à celles du cyprès. Les fleurs form un chaton écailleux qui donne naissance à un p cône qui devient charnu en mûrissant, comm fruit du Genévrier.

Cet arbrisseau, toujours vert, c'est la SABINE ( niperus Sabina), autrement dite Genévrier-Sabi Savinier, de la famille des Conifères. Toute. plante et particulièrement les rameaux sont dc d'une odeur pénétrante, peu agréable, et d'une. veur amère, âcre, résineuse. Son principe actif une huile essentielle, analogue à celle du Genévır mais qui semble plus excitante.

On ne doit l'employer qu'à doses très-modéri par exemple, l'infusion de 2 à 8 grammes de feu fraiches par litre d'eau, prise en petite quan est la meilleure préparation. Elle peut être 1 dans les affections goutteuses et rhumatism 
oniques, sans inflammation. La décoction contrée est utile pour aviver les ulcères et favoriser r cicatrisation.

es propriétés excitantes de la Sabine sont bien nues dans les campagnes; les femmes abusent vent de ce remède, qui demande à être employé c beaucoup de circonspection. Elles feraient bien ne jamais en faire usage sans avoir consulté le lecin.

oici un plant de Morelle (Solanum nigrum), elée quelquefois Morette, Crève-chien, Herbe aux yiciens, Raisins de loup. C'est une Solanée anlle, qui croit spontanément le long des chemins, les décombres et dans les terrains arides. Pour ultiver, on la sème au printemps.

a tige herbacée, anguleuse, est haute de cinnte centimètres. Les feuilles pétiolées, lisses, rement dentées, sont d'un vert sombre, elles exint une odeur un peu fétide. On voit paraitre uillet-août les fleurs blanches, petites, disposées corymbes renversés. Elles donnent naissance à fetites baies qui deviennent rouges et noires en rissant.

es baies de Morelle sont légèrement narcotiques, is il en faudrait manger une grande quantité $r$ en éprouver des accidents sérieux. Cependant 13 pourraient être dangereuses pour de jeunes rints. La plante est inerte quand elle est jeune, rueut même en manger les feuilles ; mais à l'aune, époque de son entier développement, on lui ive une vertu narcotique faible, qui augmente tpeu par la dessiccation. On emploie avec avanx la décoction très-chargée en lotions sur les 
parties enflammées, douloureuses. Le cataplası, préparé avec les feuilles calme la douleur des i cères irrités, des hémorrhoïdes, des brûlures; c' un sédatif peu énergique, il est vrai, mais que l'i trouve toujours sous la main, et qui suffit dans us foule de cas peu graves, dans lesquels il suffit d'c. tretenir une chaleur douce et humide, tout en exiçant une action sédative ou narcotique modérée.l ne faut pas lui demander plus que cela.

Puisque nous rentrons par le jardin, laissez-m avant de vous séparer, vous parler d'une plante téressante que vous connaissez tous, la Prvo (Poenia officinalis). Elle est de la famille des 1 nonculacées, dont nous aurons à parler plusieıs fois, car elle fournit plusieurs plantes médicinas très-actives. La Pivoine s'appelle vulgairem $t$ Pione, Rose de Notre-Dame, Rose bénite, He $\theta$ Sainte-Rose, Fleur de Mallet. Cette plante vive se trouve à l'état sauvage dans les prairies et s bois montueux du Centre et du Midi, et on la cult e partout pour ses belles grandes fleurs terminais, c'est-à-dire situées à l'extrémité de la tige, forms d'un calice à cinq sépales ou divisions, d'une rolle à cinq grands pétales obtus, d'un rouge vlacé, de deux à cinq ovaires ayant chacun son sile mate, et de trente à cent étamines, qui, dans a plante cultivée, se changent en pétales pour fory une fleur dite double. Car telle est l'origine de is fleurs, et si vous examinez avec soin une Rose, vis verrez, au centre, la gradation de l'étamine au tale.

Les rameaux légèrement rougeâtres, striés, n peu anguleux, partent d'une tige souterraine t/ 5 - 


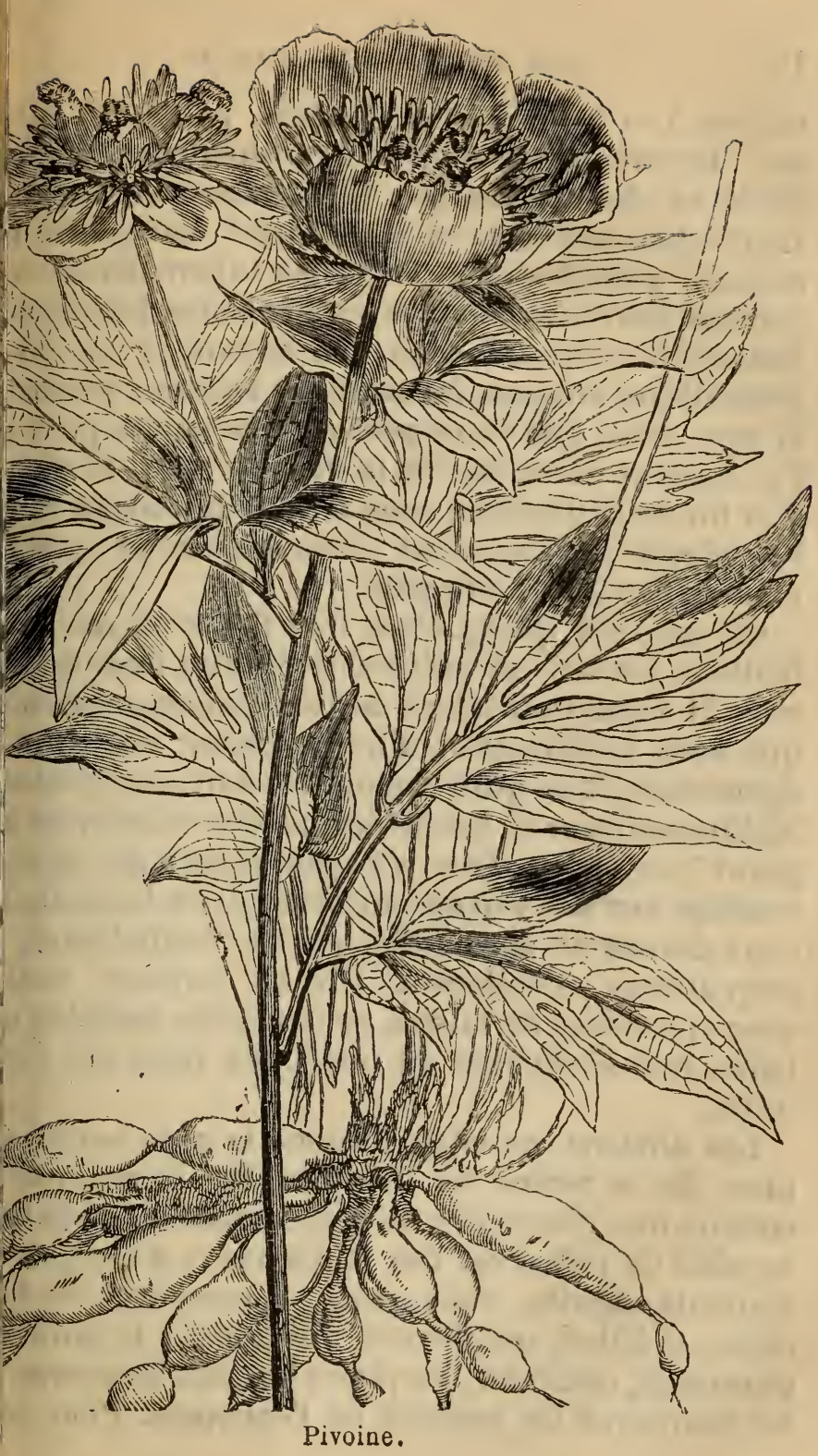


courte. Les feuilles sont alternes, pétiolées, épa. ses, bipennées, à divisions profondes, d'un $v t$ foncé en dessus, un peu blanchâtres en desso Leur odeur est forte et légèrement nauséeuse. I racines, qui affectent une forme tuberculeuse, sit grosses, charnues, rougeâtres en dehors, blanches dedans, imprégnées d'un suc laiteux, d'une od, aromatique vireuse, forment un faisceau autour la tige, ainsi que vous pouvez voir sur ce pied c j'ai dégarni de terre. On la récolte, pour la séch à la fin de l'automne, mais il vaut beaucoup mici l'employer fraîche; c'est alors seulement que 1 peut se fier à son efficacité.

On se sert quelquefois des fleurs et même : feuilles, mais il est préférable de s'en tenir à la cine. Il serait imprudent de l'administrer autrem que sous la direction d'un médecin, car c'est agent énergique qui a donné lieu à divers accider Malheureusement les médecins des villes n'en fi point usage, et l'on n'a pas encore de donn exactes sur ses vertus curatives dans les malad convulsives et spasmodiques, la coqueluche, convulsions, l'épilepsie. On l'a surtout vant, avec apparence de raison, contre cette dernière $n$ ladie, non-seulement de nos jours, mais dès l'ar. quité.

Les anciens, en effet, avaient la plus haute o nion de la racine de Pivoine. Ils la regardai comme une plante divine, une émanation de la lu capable de préserver des malheurs et d'éloigner mauvais esprits, mais pour en ressentir les bc effets, il fallait qu'elle eût été cueillie la nuit. $E_{3}$ guérissait, disait-on, les plaies les plus dangereus, los morsures de serpent et l'épilepsie. Pour ces 
rnière maladie, il suffisait même de la porter en ulette! Vous riez, et cependant, il ne manque s de gens qui, de nos jours, soient aussi crédu- Les graines de Pivoine figurent dans les offies, parce qu'il se présente pour les acheter des res qui en font des colliers destinés à préserver rs enfants des convulsions. Nous sommes, hélas! n arriérés, au dix-neuvième siècle! Il y a encore ; gens qui croient qu'un crapaud desséché, porté - le corps, préserve de la peste; qu'un fil rouge vient les saignements du nez; que la cire à cater guérit la dyssenterie; qu'il suffit de porter in soi un marron d'Inde pour éviter les hémorides et des graines de Pivoine pour écarter les avulsions !

'herchons donc à comprendre bien la nature. irtons le merveilleux, demandons à l'expérience sonnée des faits indiscutables; aux superstitions retenues par la crédulité, opposons des préceputiles; aux amulettes, des remèdes: à l'ignoce, le savoir; aux ténèbres, la lumière. 


\title{
DEUXIEMME SÉRIE DE PROMENADES
}

\author{
(JANVIER, FÉvrier, MARS.)
}

A cette époque de l'année, nous ne pouvons mes amis, compter sur une ample récolte de pla1 Cependant nous trouverons moyen de faire $q$ ques promenades fructueuses. Nous nous occ rons des végétaux qui conservent pendant l'h leur parure de feuillage, nous récolterons écorces, et bientôt, épiant le premier réveil c nature, nous cueillerons des bourgeons et sou terons la bienvenue aux fleurs hâtives qui de cent le printemps.

J'ai l'intention, aujourd'hui, d'appeler sur votre attention sur plusieurs écorces intéressar Mais il est bon de vous dire d'abord quelques $r$ au sujet des écorces en général et de leur rôle ( la végétation.

Si vous coupez un arbre, un jeune Chêne, exemple, vous remarquerez une différence not entre divers points de la partie tranchée.

Au centre, on voit une partie blanchâtre, éto c'est la moelle, formée d'un tissu lâche, spong et sans fibres. Puis viennent on B des cour de couleur foncée, dures, composées de fibres 1 résistantes, qui constituent le corps de l'arbr bois proprement dit. Ensuite paraissent des cour également fibreuses, mais d'un tissu lâche, résistant (A), c'est l'aubier, qui constitue un de mauvaise qualité. Autour de l'aubier se tro l'écorce $(\mathrm{E})$, formée en partie de tissu fibreux, $n$ 
varie beaucoup dans sa structure, comme le ntre l'apparence si diverse des végétaux. n distingue dans l'écorce l'épiderme, pellicule ce qui se dessèche, se fendille et se détache fament, mais se reproduit aussitôt. Au-dessous l'épiderme so trouve le suber, partio non fiuse, qui se développe parfois d'une façon remar-

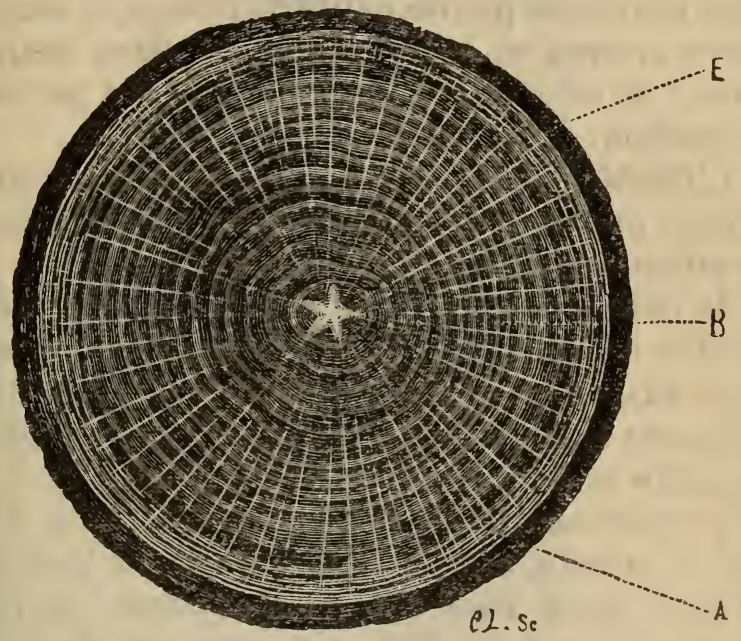

Coupe du tronc d'un jeune chêne.

le, comme dans lo Sapin et surtout dans le e-liége. Enfin, entre le suber, que l'on peut ler première écorce, et l'aubier, on rencontre ier ou seconde écorce. Celle-ci a pour caractère hctif d'être ordinairement formée de fibres plus ies, plus minces et plus tenaces que celles de ler et du bois. C'est du liber que l'on retire pres textiles du chanvre et du lin. Quelquefois tient de l'amidon, de la gomme, et peut être déré comme alimentaire. 
Si vous regardez attentivement la section jeune arbre, vous verrez que les diverses cou dont nous venons de parler sont séparées par tres couches, ordinairement assez minces, qui toutes criblées de petits trous, et que l'on obs ces trous même dans la partie la plus compact bois. C'est que les plantes sont traversées dans 11 leur longueur par de petits canaux, qui sont $c 0$. leurs artères et leurs veines, et dans lesquelsi culent la séve et les liquides élaborés par sa tı formation.

L'Erable jeune est un des arbres où l'on pe mieux observer la constitution des végétaux je viens de vous donner une idée.

Je conserve à cet effet une section de tronc Erable de deux ans, et je l'ai apportée pour vc faire examiner. Vous voyez que l'on distingu cilement le bois de la première année (accolac celui de la deuxièmo année (accolade 2) et les ties constituantes de l'écorce (accolade 3). Au c est la moelle, entourée de vaisseaux bien dist (TEM), puis viennent trois couches de fibre gneuses ou bois (FB), séparées par des vaiss (VP). La même succession se répète pour lt formé pendant la seconde année. Puis viennes fibres corticales, ou liber (EC), le suber (I enfin l'épiderme (EP).

Telles sont les notions élémentaires dont avez besoin pour bien comprendre les observe que nous ferons aujourd'hui.

Voici tout près de nous un premier sujet servation. Vous connaissez cet arbre, lo l (Fagus sylvatica), autrement dit Fau ou Fa 
la famille des Cupulifères. C'est un des arbres plus beaux et les plus utiles de nos forêts. Son

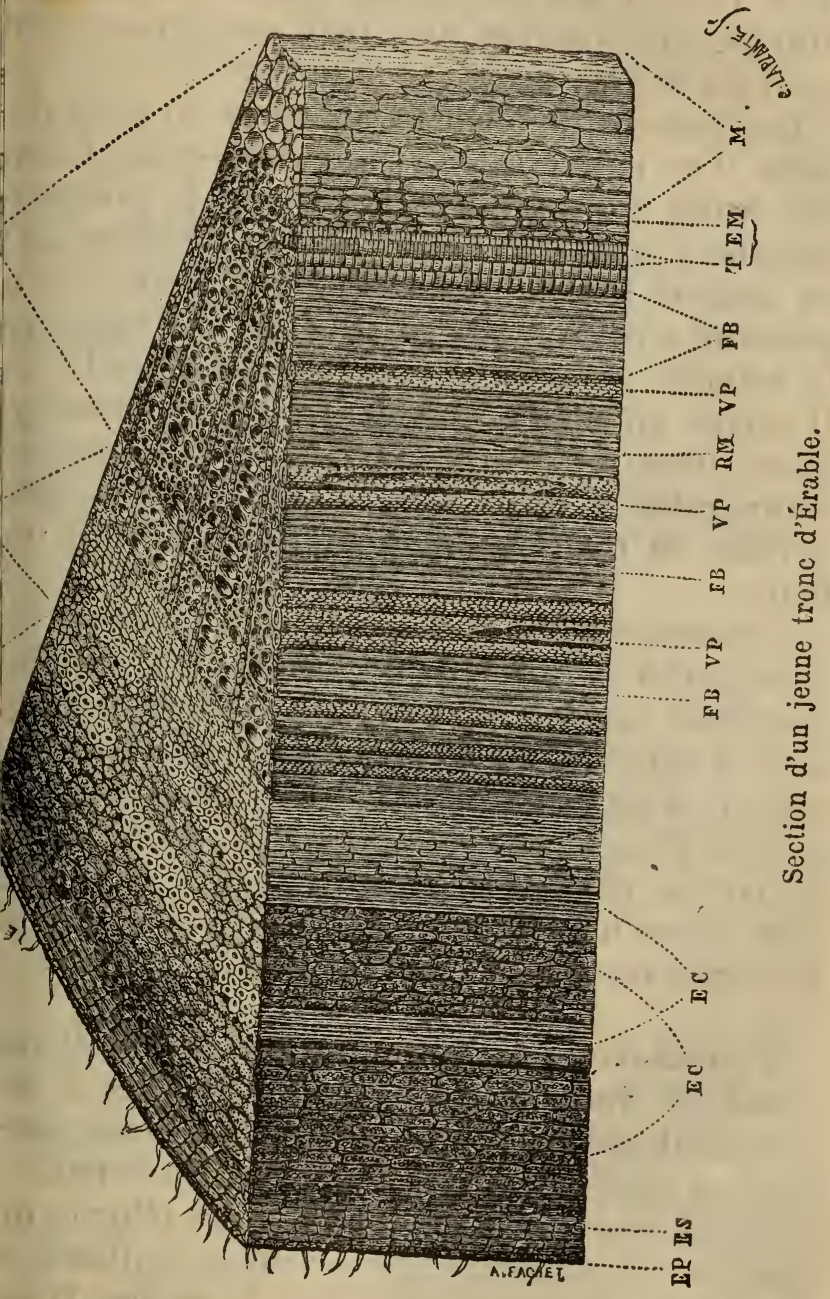

est estimé pour la menuiserio et le charron- 
nage, on en fait des brancards, des jantes de rov des vis. Il dure très-longtemps dans l'eau, tal qu'à l'air il est facilement piqué des insectes] fournit un charbon excellent, supérieur mêm celui du chêne.

Les graines nommées faînes ont un goût de sette. On les récolte en automne pour fabrig une huile comestible qui jouit de la propriété ne rancir que très-lentement. Les tourteaux c on extrait l'huile peuvent être donnés en $p$ quantité aux animaux ruminants, aux cochons la volaille, mais ils sont un poison pour le che Il existe, en effet, dans la graine, un principe létère inconnu qui produit chez l'homme un comparable à celui de l'ivresse; il faut dono garder de manger une grande quantité de fruits.

L'écorce est douée d'une saveur âpre due pri palement à la présence du tannin. On l'emploi décoction comme fébrifuge, à la dose de $30 \mathrm{gr}$ mes d'écorce fraîche ou $15 \mathrm{gr}$. d'écorce sèche $200 \mathrm{gr}$. d'eau, administrés une heure avant l'ac A dose plus forte, elle agit comme vomitive et gative, et il peut y avoir avantage à obtenir effet. C'est un remède qu'il est bon de conna bien que nous en ayons de plus énergiques.

Pouvez-vous me dire à quoi sert le Pomm Vous ne vous êtes jamais demandé cela, tou croquant ses fruits, verts ou mûrs, dans votre clos, et peut-être aussi dans celui du voisin.

Vous savez déjà que le Pommier (Pyrus ma comme la plupart de nos arbres fruitiers, at tient à la grande famille des Rosacées. Il est 


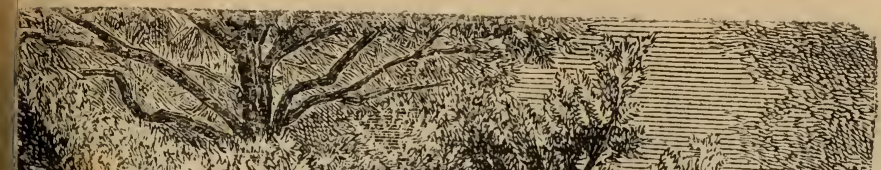
(15)

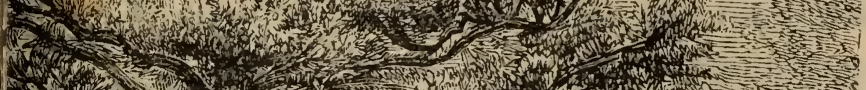
1.1. (1)

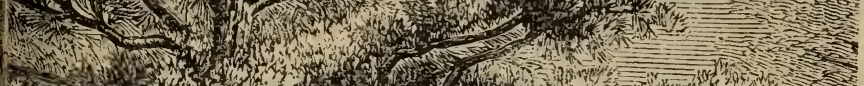

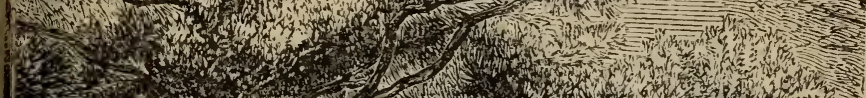

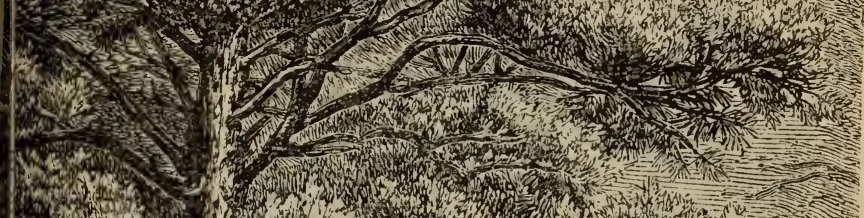

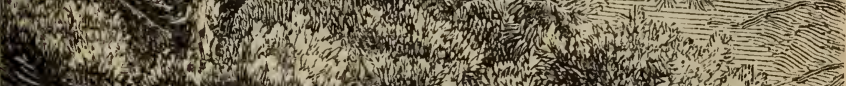

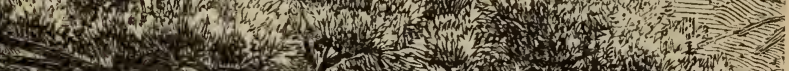

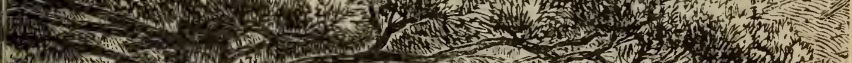
137.

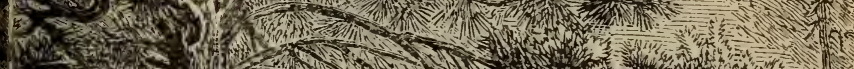
160.

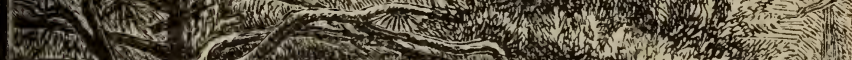

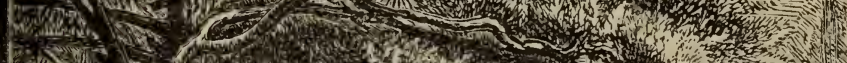

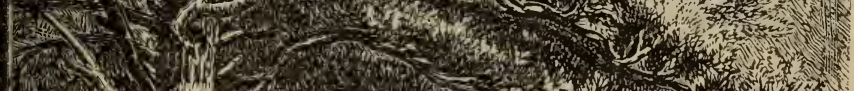

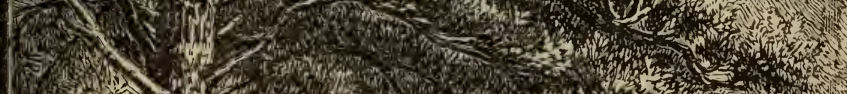

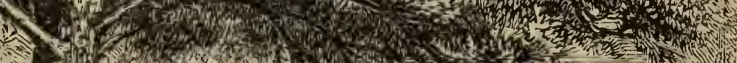

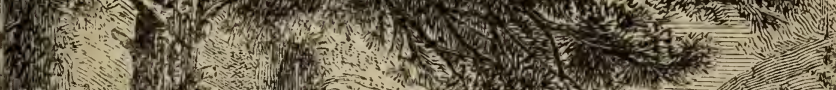

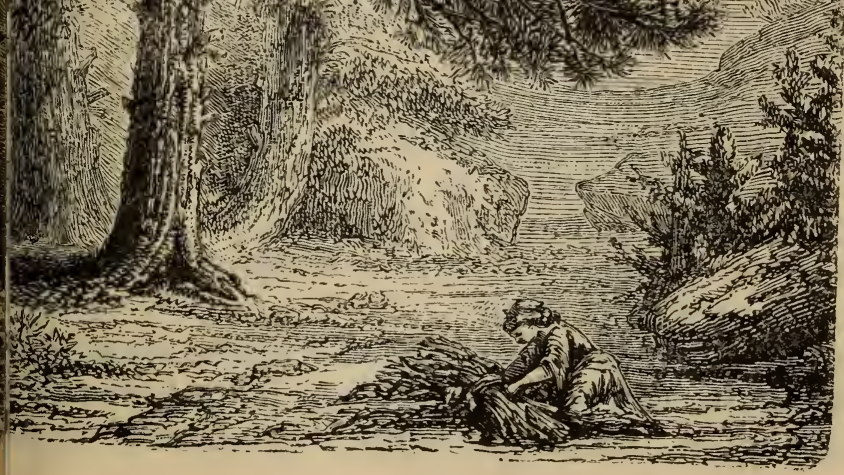


tile de vous le décrire. Celui qui croît naturell ment dans nos forêts diffère beaucoup de celui $q$ vous est familier. Celui-ci a perdu par la cultu ses épines, tandis que les fruits ont considérab. ment gagné en grosseur et en saveur. Les semis

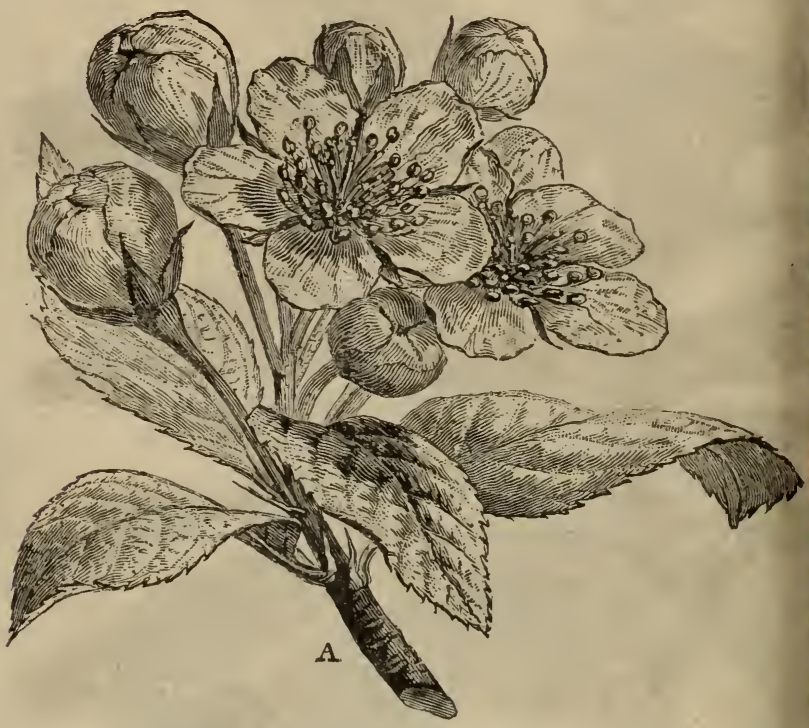

Fleurs de Pommier.

la greffe aidant, on possède aujourd'hui plus 200 variétés de pommes douces ou aigres.

La pomme, avant sa maturité, est acide, âpr elle contient une espèce d'amidon, qui se transfor ensuite en sucre, en même temps que lo princi astringent se medifie.

Les pommes de reinette et d'autres variétés do ces sont usitées journellement pour préparer, $\mathrm{F}$. décoction, une tisap rafraîchissante utile dans 
aladies inflammatoires. Avec les pommes aigres, - fabrique le cidre, boisson ordinaire dans le rd-ouest de la France. On a remarqué que cette isson préserve s maladies calcuises. Je ne vous rlerai que pour śmoire du sucre pomme préparé ec le suc épaissi pommes chois, additionné de re ; c'est une andise plutôt jun médicament. Imployée en détion, l'écorce iche de la racine Pommier à la lie de 60 gramrs dans 200 gram rs d'eau, coupe r;-bien les fièis d'accès simis, surtout si l'on précéder son roloi par un lévomitif suivi (a purgatif. De (nbreuses expéi ices confirment

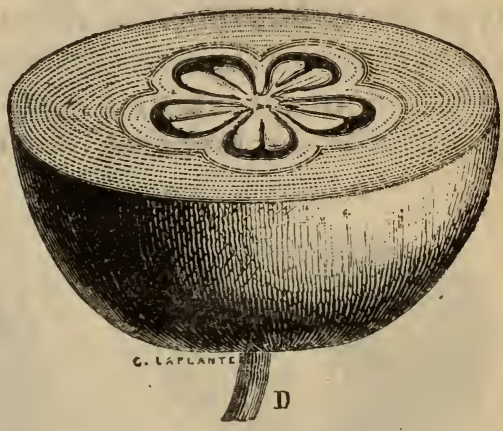
premières observations recueillies à ce sujet, et c extrait de l'écorce du Pommier une substance cumée phloridzine, qui peut, dans beaucoup de 
cas de fièvres quotidiennes et tierces, remplacer sulfate de quinine.

Le Marronnier d'Inde (Assculus hippocastanun type de la famille des Esculacées, nous offre I autre exemple de ces fébrifuges incertains dans leu effets, dont on a tour à tour trop vanté puis nié 1 propriétés. Je tâcherai de vous expliquer ces an
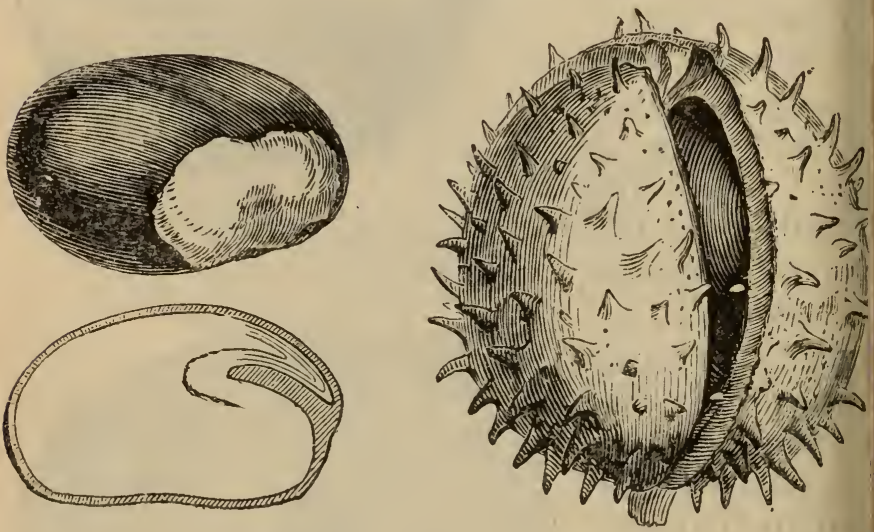

Marrun et son enveloppe.

malies lorsque nous nous occuperons du Saule, meilleur des fébrifuges indigènes. Aujourd'hui d sons quelques mots du Marronnier.

C'est un arbre d'Asie, parfaitement acclimaté । Europe, où il fait l'ornement des parcs et des jardin par son port élégant, ses belles feuilles palmées q semblent formées de sept feuilles réunies à l'extr mité d'un même pétiole. A ses grappes de fleurs r cherchées des abeilles, succèdent des fruits sembl bles à la châtaigne et qui mûrissent à l'automn L'écorce des jeunes rameaux, que l'on doit $r$ 


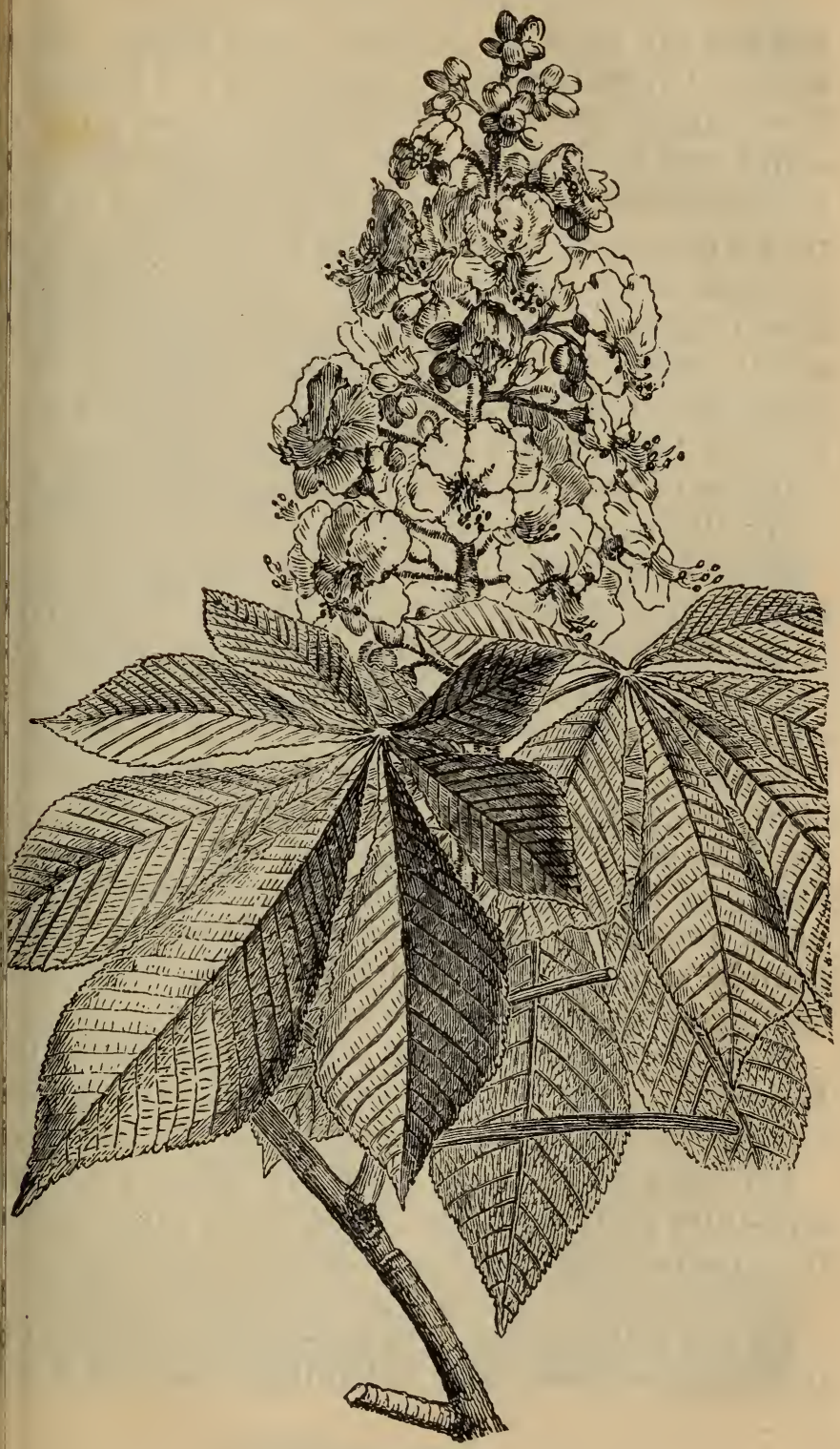

|Branche de Marronnier $\left.\right|^{\prime}$ 
cueillir au mois de février, a une saveur âpre| amère. On en fait une décoction de 2 à 3 onces I litre d'eau, que l'on prend par tasses, pour p duire une action légèrement tonique et astringen Comme fébrifuge, il faut administrer 12 ou 15 gra mes d'écorce en poudre avant l'accès, dans du $\mathrm{m}$ ou sous toute autre forme. Une décoction conce trée d'écorce fraîche remplirait le même but, serait probablement plus efficace, attendu que dessiccation semble diminuer notablement ses pr priétés. Jusqu'ici on doit regarder l'écorce de Ma ronnier comme un agent douteux, qui appelle nouvelles expériences.

Mais, ce qui est incontestable, c'est que l'on pe tirer bon parti des marrons d'Inde. Ceux-ci re ferment 30 pour 100 d'une excellente fécule, aus bonne que celle de pommes de terre. Mais elle e unie à un principe amer, âcre, dont il faut la d barrasser. Pour cela, on réduit les marrons pulpe, on y ajoute, par 100 kilogrammes, 1 ou $2 \mathrm{k}$ logrammes de carbonate de soude dissous dans l'eau; on mélange, on laisse macérer pendant que ques heures. Il suffit alors de laver la pulpe grande eau, pour recueillir, par précipitation, ur fécule parfaitement pure.

Il est à désirer que l'on utilise ainsi les fruits d Marronnier, plutôt que de les donner aux moutor et aux chèvres, qui ne peuvent s'en trouver bien.

On fait avec la fécule amère du marron une coll très-utile pour les grands herbiers, parce que le insectes ne l'attaquent pas.

Ce joli arbuste toujours vert, à tiges un peu ram pantes, rameuses, dont le feuillage ressemble à ç 


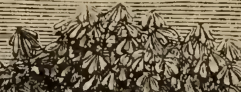

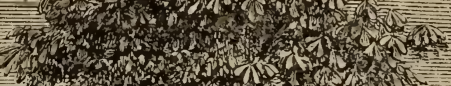

1 (1) W

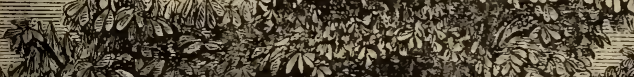

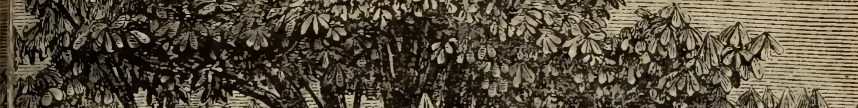

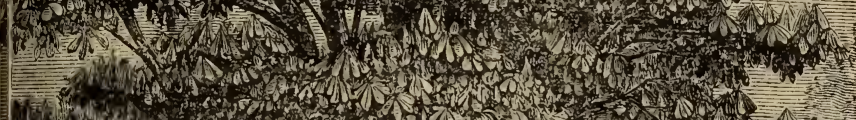

20 (2)

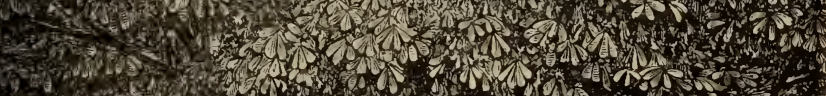

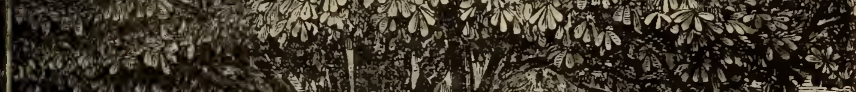
2. 65 5 .

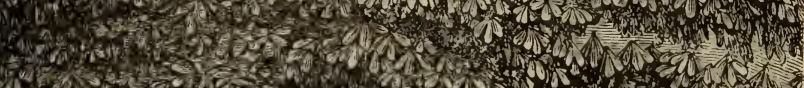

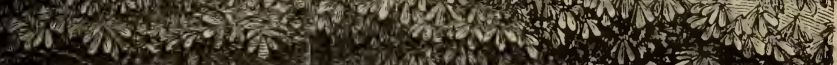

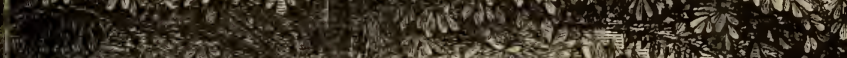

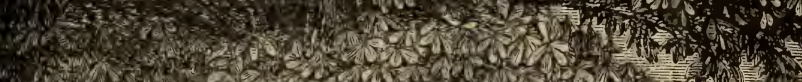

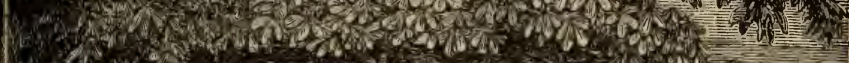
(196) G 
lui du buis, ce qui lui a valu son nom, c'est la $1_{3}$ SEROLE (Arbutus uva ursi), de la famille des 1 cacées ou Bruyères. On l'appelle aussi Arbou traînant, Bousserole, Petit buis, Raisin d'our: croît spontanément dans le midi de la France, s tout dans les montagnes. Il aime les terrains $p$ reux et ombragés. Pour le cultiver dans le Norc faut garder dans l'orangerie pendant l'hiver jeunes plants, provenant de semis ou de boutures ne les placer en pleine terre qu'à l'âge de trois : En avril-mai paraissent ses fleurs rosées, dispos en grappes inclinées à l'extrémité des rameaux. fruits sont des petites baies rouges oblongues.

La corolle est d'une forme particulière, dite

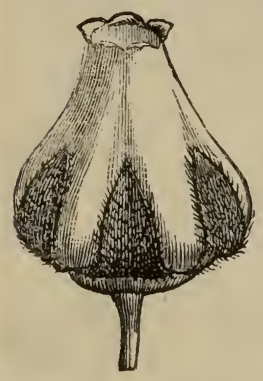
céolée, dont le type est fourni un grand arbuste de la même mille, l'Arbousier (Arbutus unec dont les fruits, qui mûrissent automne, ressemblent à des frais Ce genre de corolle, commun di les bruyères, a l'apparence d'un v conique dont la base renflée est e brassée par le calice, et dont l'c Fleur d'Arbousier. fice étroit est découpé en dents courbées.

L'écorce, les feuilles et les fruits de la Busser sont diurétiques et légèrement astringents. On e ploie surtout la décoction des feuilles à la dose 30 grammes par litre d'eau. Cette plante est $\mathrm{\imath}$ des plus efficaces dans les maladies des voies u naires. Elle guérit souvent les difficultés d'urin accompagnées de catarrhe de la vessie, et soula toujours les malades qui souffrent de la gravel La Busserole n'agit point, dans ce oas, par u 
tu spécifique, mais elle exerce une action locale n marquée sur les organes affaiblis et leur pert d'opposer à la maladie une réaction suffisante. a action légèrement tonique sur le système nerix l'a fait employer avec succès pour combattre lébilité extrême qui accompagne certaines affecis chroniques des voies respiratoires, mais elle aucun pouvoir contre la phthisie véritable, qui lique la présence de tubercules dans le poumon. is ce cas les toniques n'agissent que comme palifs; ils ne guérissent pas, mais peuvent prolonde beaucoup l'existence du malade.

Tous savez tous le nom de ces grandes rosettes tes, un peu semblables à des têtes d'artichaut, ement plein de grâce dont la nature pare toute née les toits de chaume. A ses feuilles épaisses et rnues, vous reconnaissez une plante de la famille Crassulacées, qui remplacent, dans nos climats, Cactus des régions chaudes. On la multiplie facilent de drageons que l'on plante dans un terrain sec. a Joubarbe (Sempervivum tectorum), nommée si Grande Joubarbe, ir la distinguer du lon brûlant ou PeJoubarbe, dont nous ns parlé dans notre nière promenade, est nmée aussi Herbe cors, Artichaut sau;e. Elle fleurit en liet-septembre. Les lirs roses, purpuri-

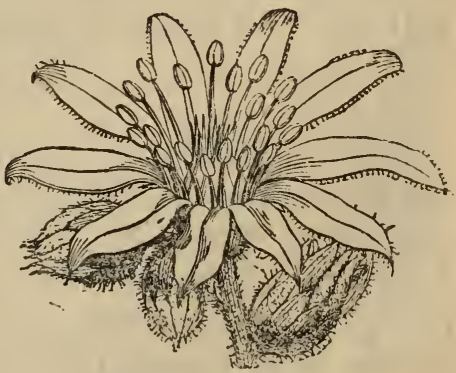

Fleur de joubarbe. 1:, sont disposées en corymbe irrégulier à l'ex- 
trémité d'une grosse tige simple garnie de feuil; moins grandes et plus minces que celles de la ba celles-ci sont les seules dont on fasse usage. El sont plus actives avant et après la floraison. Li, saveur est à la fois fraîche et un peu astringer.

Le suc de Joubarbe et ses feuilles pilées calmi l'irritation des dartres vives, des ulcérations, "I brûlures, soulagent les maux de têtes fébriles, $m$ dans tous les cas il est nécessaire d'en renouve souvent l'application. Le nom d'Herbe aux cors a été donné à cause de son emploi populaire p détruire ces callosités de la peau. Il y a, vous savez, une foule de remèdes contre les cors : feuilles de Souci, de Chou rouge, de Lierre, petite Joubarbe; les uns s'emploient à l'état na rel, les autres macérés dans du vinaigre. Il i porte, surtout, de maintenir pendant longtemps s le cor une compresse humide, rendue active ? un acide qui aide au ramollissement de la peau durée, la gonfle, et permet de l'enlever sans dc leur. Si le remède est légèrement caustique, il pe détruire les parties profondes du cor, et prével sa reproduction, à la condition que la chaussure soit pas une cause constante de récidive.

En attendant que le soleil printanier fasse éclc les premières fleurs, nous allons nous occuper a jourd'hui d'écorces, de bourgeons ou de plant toujours parées de verdurè. Le réveil de la sé donne déjà des vertus plus énergiques aux parti jeunes des vegétaux, c'est le temps propice po recueillir plusieurs produits précieux.

Yoici un arbre familier, lo Bouleau (Betula alb. 
toe de la famille des Bétulinées, qui mérite quel, ces moments d'attention. On Ippelle aussi Bouleau blanc, Ijle, Bouillard, et du temps où In croyait qu'un bon faisceau verges était indispensable à la d cipline d'une classe, on l'avait ynommé le Sceptre des maîs d'école. Le nom et la chose it heureusement tombés en bli.

e Bouleau se contente de toules expositions, de tous les rains, et croît dans les régions déales jusqu'aux limites de la gétation des arbustes. Les illes ont une odeur agréable, lo saveur un peu amère et asngente, leur décoction est lé-ement diurétique et excitante, on l'emploie dans le Nord nme dépurative et anti-rhutismale. Les bourgeons ont - propriétés plus énergiques.

a partie la plus intéressante l'écorce. L'épiderme, trèsis, se lève par couches argen$s$ enduites d'une résine en pou. ; aussi on l'emploie pour faire torches. Les Suédois, les ons, couvrent leurs cabanes c l'écorce de Bouleau, ils en t des corbeilles, des cordes,

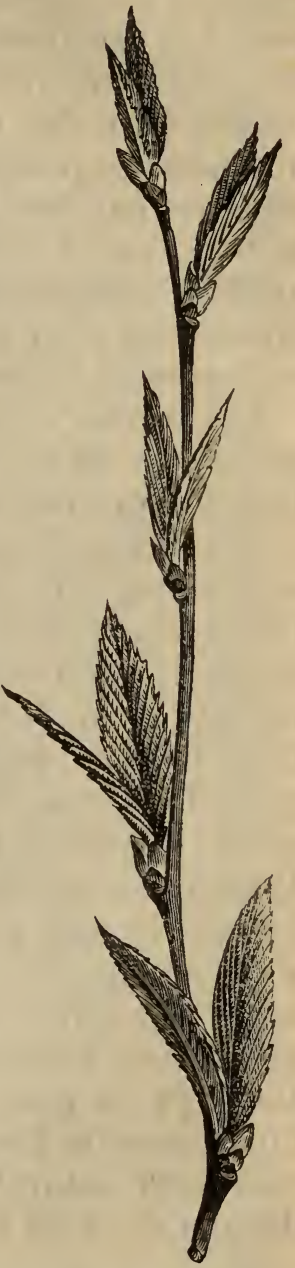

Bourgeons de Bouleau. vases capables de contenir des liquides, $\mathrm{Au}$ 
Kamtchatka, on mange la seconde écorce et on prépare une sorte de bière; l'épiderme sert de ] pier. La distillation de l'écorce de Bouleau four une huile empyreumatique employée en Russie : préparation des cuirs, auxquels elle communio une odeur agréable, et la propriété de n'être po attaqués par les insectes. Enfin l'écorce, comme feuilles, sert à teindre en jaune. Quant à ses $p$ priétés médicales, ce sont à peu près celles bourgeons; on les met surtout à profit dans maladies de la peau et les affections scrofuleuses

Les jeunes Bouleaux servent à faire des cerc: des jantes de roue, des sabots, avec les branches fabrique des balais.

Les habitants du Nord, moins favorisés que ni sous le rapport de la diversité des végétaux uti] tirent également parti de la sève sucrée de arbre. Un peu avant le développement des feuil] on fait dans le tronc, au moyen d'une vrille, trou un peu profond dans lequel on enfonce bout de roseau, et l'on dispose au-dessous un v pour recevoir la sève qui coule par cette blessu Quand elle ne tombe plus que goutte à goutte, bouche le trou avec un fausset pour que l'arbre s'épuise pas. Ce liquide donne, par l'évaporati un sirop qui remplace la mélasse. On en prép. aussi une boisson fermentée. C'est ainsi que da les pays les plus dénués, sous le climat le plus ru l'indu strie de l'homme découvre et met à profit rich esses naturelles que l'on ignore ou que l'on daigne dans les régions plus favorisées.

Pendant que nous nous reposons au pied de Chêne, je vais vous faire en peu de mots l'histo 


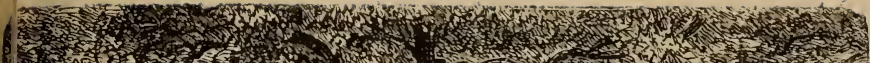

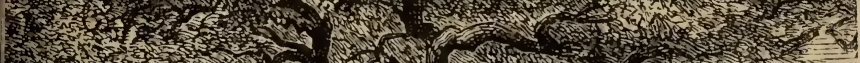

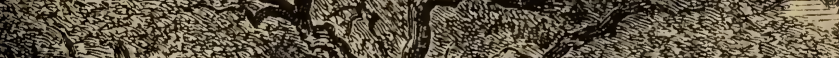
H.

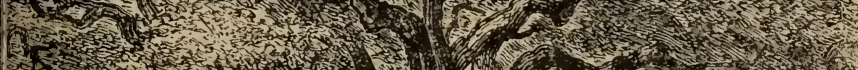
3.

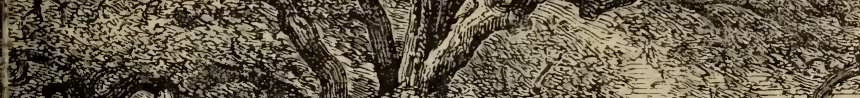

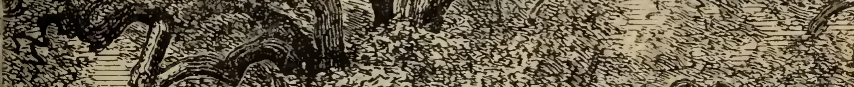

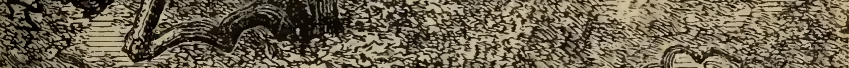
1.7.

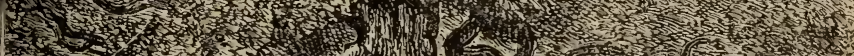
1.7.

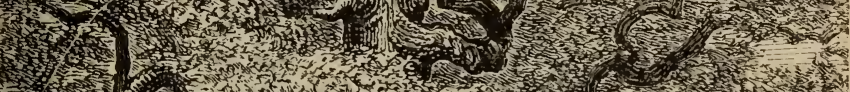

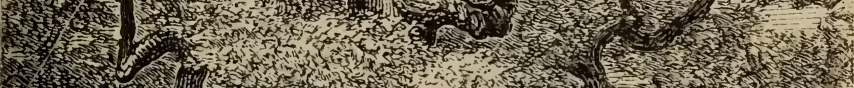

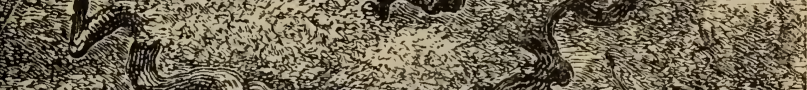

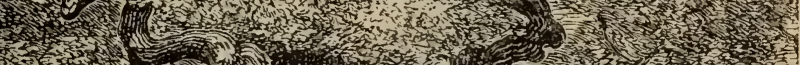
(1)

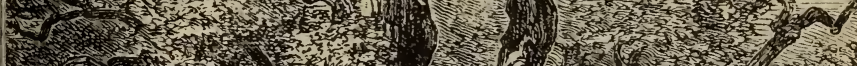
I.

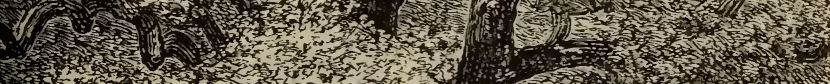

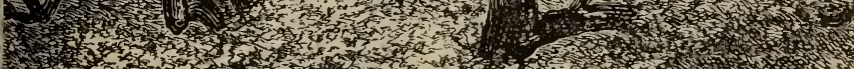

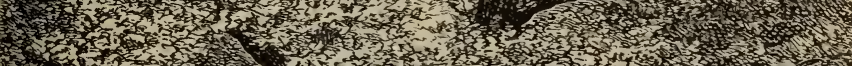

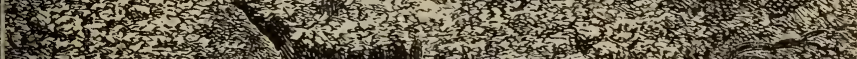
(1)

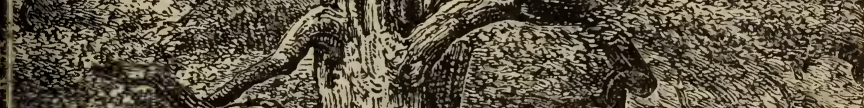

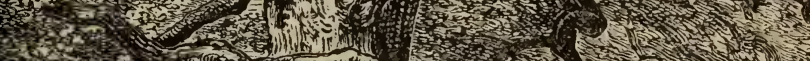

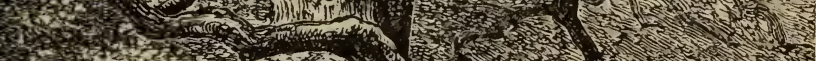

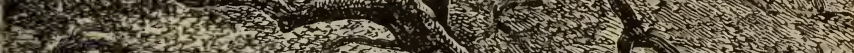

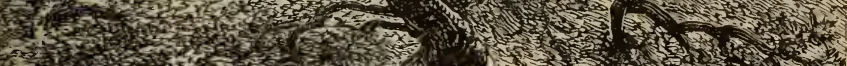

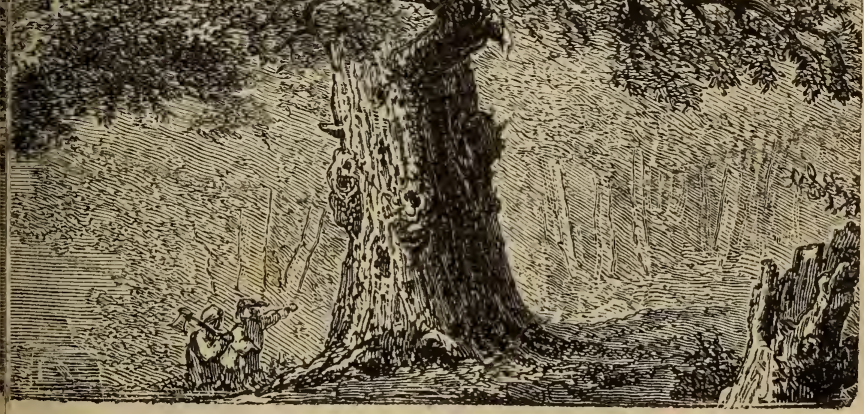

Chêne. 
de ses qualités. On le considère à bon droit con le plus utile et le plus bel ornement de nos fors,

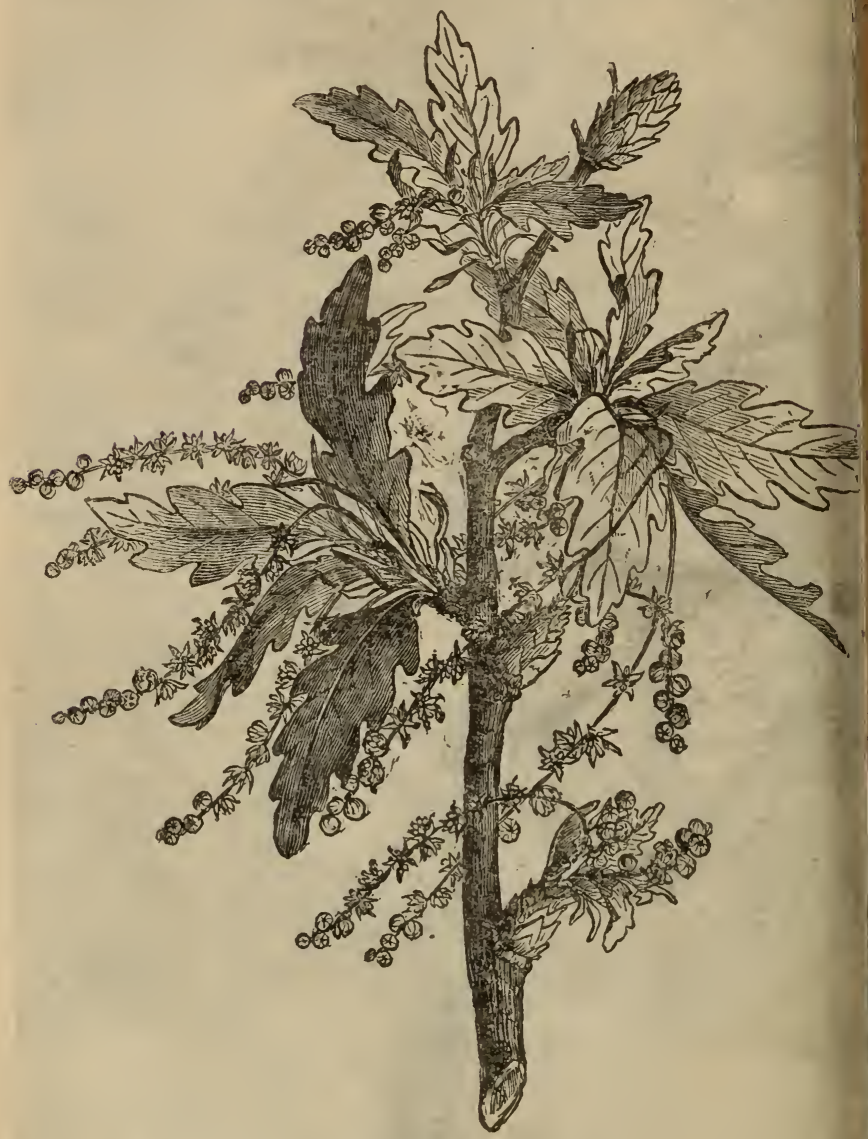

Fleurs mâles de Chène.

où il acquiert des dimensions énormes. Son b solide et durable est recherché pour la charpen les contructions navales, le charronnage et la $\mathrm{m}$ 
uiscrie. Le CHÊNe, ou Chêne-rouvre (Quercus bur) est de la famille des Cupilifères, comme le être, le Coudrier, le Charme, le Châtaignier. On la istingue par sa floraison remarquable. Les fleurs âles en chatons allongés sont composées d'étaines et d'un calice.

Les fleurs femelles sont réunies par deux ou trois

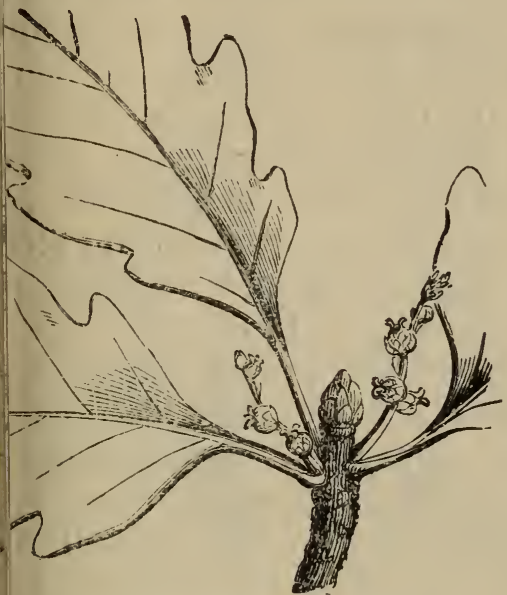

Chêne. - Fleurs femelles.

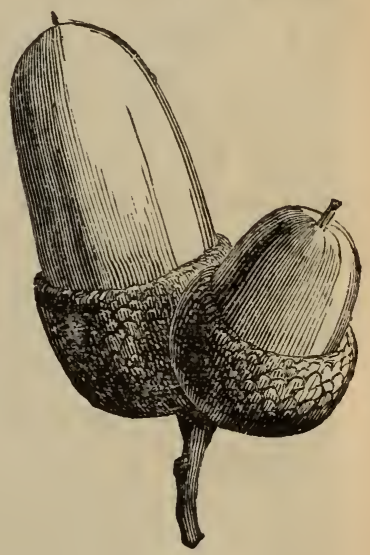

Fruit.

Ins une enveloppe commune qui plus tard s'accroît se transforme en une espèce de coupe à laquelle x zère le fruit, comme on le voit dans le gland, ou fi le recouvre tout entier, comme dans la noisette. L'écorce recueillie sur les jeunes branches de êne est lisse, d'un blanc verdâtre en dehors, roujitre en dedans. L'odeur est fade, la savour asingente, à cause de la grande quantité de tannin felle contient. C'est ce principe astringent qui la id précieuse pour le tannage. Aucune autre úrce ne donne d'aussi bons produits. 
C'est également a ses principes astringents $q$ cette écorce doit ses propriétés médicales, d'auta plus appréciables, qu'on peut se la procurer parto sans frais. Il ne faut l'administrer à l'intérie qu'avec circonspection, à la dose de 2 à 4 gramm pour combattre. les flux muqueux chroniques, a compagnés d'une relaxation générale des tisst dans la dyssenterie, les hémorrhagies scorbutiqu ou provenant de débilité des organes. Au lieu l'écorce en poudre, on peut donner la décocti préparée avec 10 à 15 grammes d'écorce broy dans un demi-litre d'eau. On a cru qu'elle ét douée d'une haute vertu fébrifuge, et lors du bloc continental, sous le premier empire, le Quinqui ayant atteint le prix énorme de 12 francs l'once, vendait soús le nom de Quinquina indigène, mélange à parties égales de poudres d'écorce Chêne, de Camomille romaine et de Gentiane. mélange eut un effet excellent pour guérir les fièvr intermittentes récentes ou anciennes, mais il n'c pas plus infaillible que les autres fébrifuges, et médecin a besoin de beaucoup de tact pour bi choisir le remède qui convient le mieux à chaque ce

A l'extérieur, l'écorce de Chênè rend aussi nombreux services. La décoction de tan (nom l'écorce en poudre) employée en lavement ou lotions, est très-utile comme astringent, dans diarrhée, la dyssenterie, les écoulements muquel lorsqu'il n'y a pas d'inflammation, màis manq de vitalité des organeś. On peut aussi en fairé $d$ gargarismes dans les cas de maladies ulcéreus de la gorge. Les personnes obligées de travail] habituellement les pieds dans l'eau, se trouve très-bien de saupoudrer leurs chaussures avec 
tan, ou de garder la nuit des bas qui en sont légè rement couverts. Les gerçures de la peau se cicc trisent et les parties ramollies se raffermissent.

La décoction concentrée de tan ou d'écorc fraîche de Chêne assainit la surface des ulcères . hâte leur cicatrisation. On peut aussi les saupot drer avec un mélange de tan et de poudre i charbon, qui, agissant à la fois comme astringen absorbant et désinfectant, est principalement uti dans les cas de gangrène, de pourriture d'hôpita et d'ulcères sanieux.

On trouve dans le commerce, sous le nom c café de glands, une poudre composée de glan torréfiés et broyés. Lorsqu'elle est bien prépar et que la fraude n'y ajoute pas une forte proportic d'orge, l'infusion de cette poudre est employ avec avantage pour combattre les accidents scr fuleux chez les enfants, ranimer les fonctions dissiper les engorgements, entre autres le carrea On sait que malgré leur amertume et leur astri gence, les glands sont fort recherchés des pou ceaux ; cette nourriture les engraisse rapidemer attendu que les fruits du Chêne contiennent $u$ grande quantité de fécule.

Vous avez remarqué sans doute sur les bov geons, les pétioles et les jeunes branches du Chê des excroissances dures, ligneuses, à surface rid ou lisse, ce sont des galles ou noix de galles. El résultent de la piqûre d'un insecte qui y dépose œuf autour duquel s'accumule peu à peu cette $\epsilon$ croissance. Un petit ver sort de l'œuf, puis transforme en mouche, comme la chenille se tra: forme en papillon, et perce la galle pour s'envol 
es noix de galles possèdent à un haut degré les ropriétés astringentes de l'écorce de Chêne. Leur nfusion donne, avec les sels de fer, un précipité leu noirâtre, et l'on utilise cette action pour fabriuer l'encre et pour la teinture en noir.

Nous avons sous la main un autre objet d'étude. oyez ce Lierre qui grimpe autour du tronc du hêne plusieurs fois centenaire et semble vouloir acher sa vétusté sous les guirlandes d'un feuillage jujours vert. Ce n'est point un parasite; il ne detande qu'un appui à l'écorce crevassée dans lauelle il enfonces ses crampons comme des racines. Toutes les parties du Lierre sont utiles. Les fruits a baies noires, de la grosseur d'un pois, qui mûssent en janvier-mars, constituent, à la dose de $x$ à douze, un purgatif très-énergique dont on juse un peu dans les campagnes. A la dose de ux grammes en poudre, elles ont guéri des fièes vernales et automnales, mais c'est un médiment qui demande de nouvelles expériences. vec le bois de Lierre on fait des pois à cautère, ii entretiennent très-bien l'irritation de la petite aie sur laquelle on maintient une feuille de la ante. Enfin les feuilles, cuites à l'eau et réduites pulpe, opèrent un changement favorable sur les cères indolents et les plaies de mauvaise nature; is cataplasmes agissent aussi comme résolutifs sur 1; engorgements froids. L'infusion d'une poignée feuilles dans du vinaigre, employée en lotions datin et soir, guérit la gale en huit à dix jours.

Il ne faut pas confondre le Lierre Grimpant ( $\mathrm{He}$ (ra helix), de la famille des Araliacées, avec le IERRE TERRESTRE (Glecoma hederacea), qui est uno 
Labiée, et dont nous aurons occasion de nous or cuper plus tard. Le Lierre commun, arbuste ram

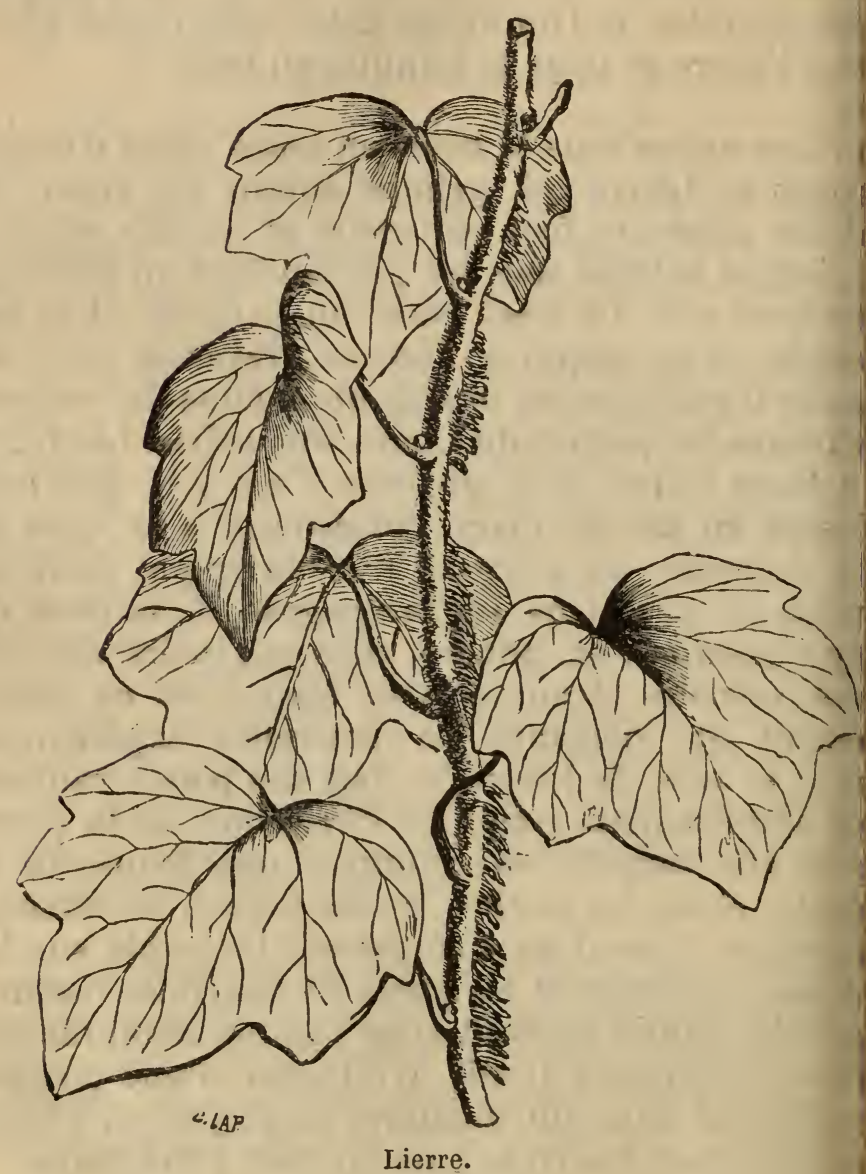

pant ou grimpant, qui croît naturellement en $\mathrm{E}_{1}$ rope et que l'on cultive principalement pour cach les murailles des jardins, ou former des bordure 
3o reconnait à ses feuilles coriaces et luisantes, ćchancrées en cœur à leur base; à ses petites fleurs l'un vert jaunâtre, disposées en corymbes globueux, et à ses fruits qui deviennent noirs en mûrisant. La tige, grêle dans nos climats, acquierț dans es régions chaudes un volume beaucoup plus conidérable. Elle laisse exsuder une gomme résine romatique dont on n'a pas encore bien étudié les ropriétés.

Le Lierre, vous voyez, n'est pas seulement desné à donner un aspect pittoresque aux murailles ues, aux ruines et aux troncs délabrés, c'est une lante vraiment utile, et comme telle nous la ferons gurer dans notre collection.

Ce bel arbre dont le tronc élancé, les rameaux uffus, un peu tombants, et le feuillage toujours rt, forment pendant la saison rigoureuse lo plus ornement de nos paysages, c'est le PIN sYListre (Pinus sylvestris), ou Pin commun, $\mathrm{Pi}$ astre, Pin sauvage. Il s'élève souvent à plus de ente mètres. On le distingue des autres arbres ujours verts de la même famille, par ses feuilles ngues d'environ cinq centimètres, dures, étroites, rmant gouttière, aiguës au sommet, réunies deux deux dans une gaîne molle. Les fleurs mâles nt disposées en grappe tronquée, arrondie, tandis e les fleurs femelles sont réunies en un chaton buleux. Les ovaires de ces fleurs, s'accroissant rès la fécondation, donnent naissance à des cônes intus, pendant's, formés d'écailles ligneuses entre quelles se trouve les graines. e Pin sylvestre sert de type à la famille des Co'res, qui comprend, en outre du Genévrier et 
de la Sabine que nous avons étudiés, les espèce nombreuses de Pins, les Sapins dont les feuill sont éparses, et les cônes formés d'écailles mince arrondies ; les Mélèzes, les Cèdres, les Cyprès.

Les Pins et les Sapins fournissent un bois exce lent, et l'on en retire un grand nombre de produi indispensables à l'industrie : la térébenthine, la $r$ sine. le brai sec, le brai gras, la poix blanche

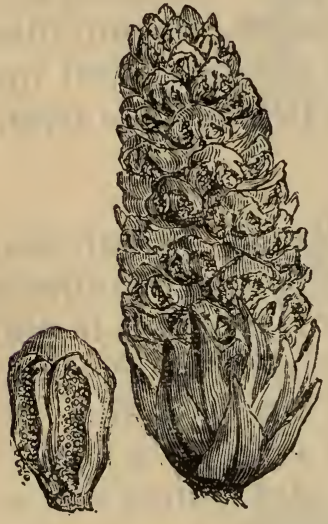

Fleur mâle.

Pin sylvestio.

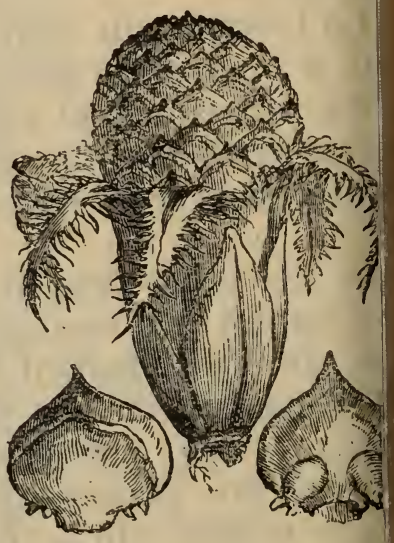

Fleur femeile.

noire, le noir de fumée, l'essence de térébenthir la benzine, des huiles essentilles légères, employé dans l'éclairage, la paraffine dont on fabrique $c$ bougies, le goudron, le vinaigre de bois, la cre sote, l'acide phénique ou carbolique. Vous voj que peu de végétaux sont aussi précieux. Mais n'est pas tout encore. Les Lapons mangent la conde écorce du Pin, qui est nutritive, tandis q la première écorce est assez légère pour rempla lo lićge des filets. Les semences du Pin d'Italie st 


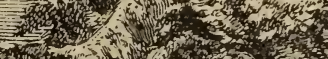

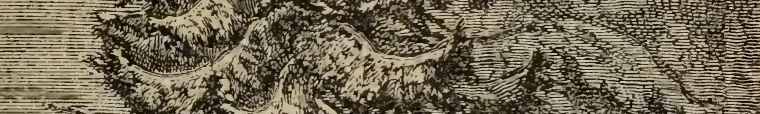

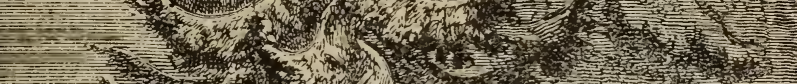

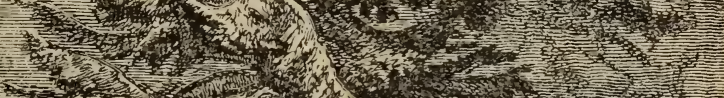

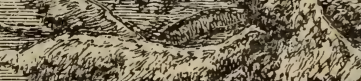

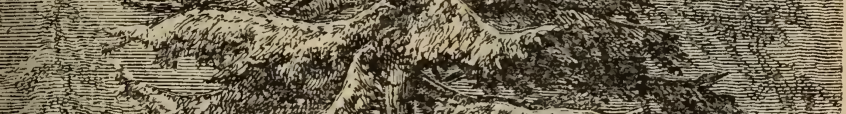
14. S. C M

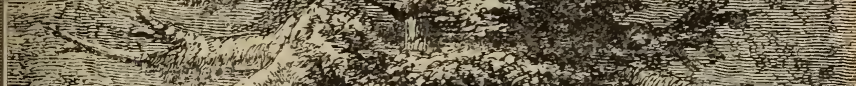
W

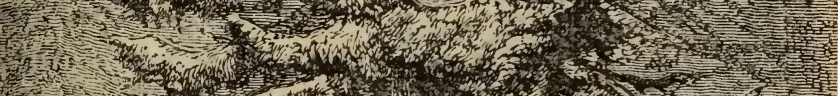

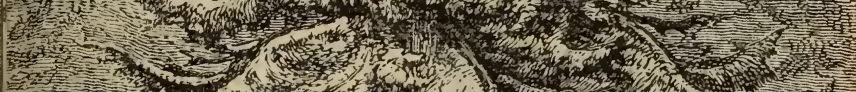

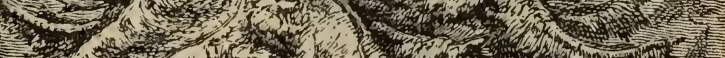
(n)

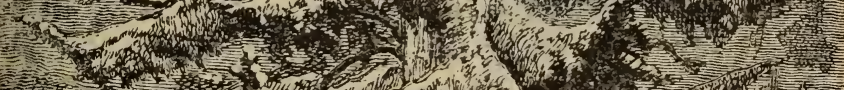

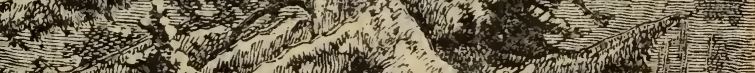
$x=1+2 y-4$

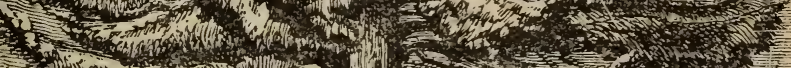

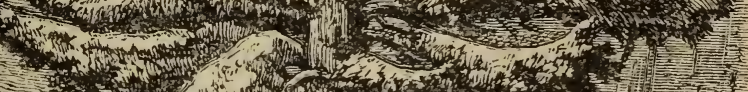

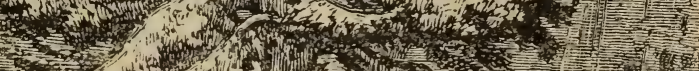
1.

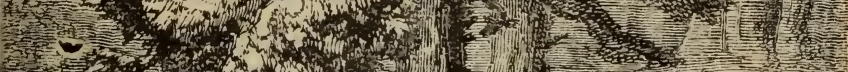
$c^{2}+2+2+23^{2}$

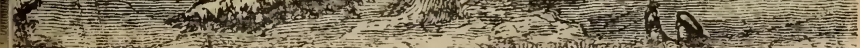


comestibles, celles du Pin sylvestre convienn aux volailles, et le cône est un excellent comb tible. Enfin les feuilles de ces arbres, bouillies d.

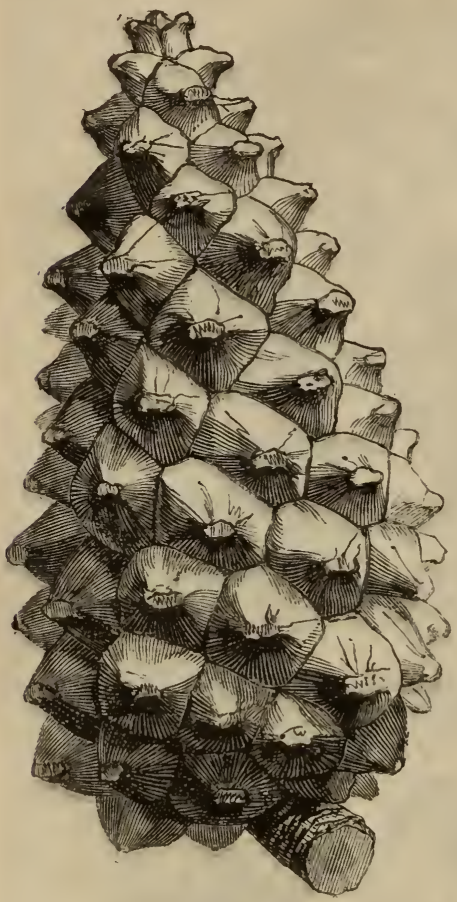

Cỏne de Pin. une solution de $c$. bonate de soude, lav. et cardées, fourniss une espèce de laine gétale dont on cont tionne des flanelles ] giéniques, des éto moelleuses et chauc et dont on peut $f \varepsilon$ d'excellents matelas: me faudrait de long heures pour vous ex] quer tout cela en dét et je dois me borne vous indiquer, par les produits des Pin des Sapins, coux sont le plus emplo, en médecine.

Les bourgeons, infusion prolongée en décoction dans l'e: le petit lait, le cidre vin, et surtout la biè à laquelle ils donnent un goût agréable, constitu un excellent remède antiscorbutique. Leurs p. priétés toniques, excitantes, diurétiques, sudor: ques, favorisent puissamment la réaction nécessa pour triompher de l'affaiblissement général c accompagne non-seulement le scorbut, mais la p] part des maladies chroniques contractées par su 
e mauvaise nourriture et d'une habitation malLa décoction concentrée avive les ulcères lents. Les fumigations dans lesquelles la vapeur l est mêlée aux vapeurs des bourgeons sont loyées avec succès dans le rhumatisme.

térébenthine que l'on recueille d'incisions $s$ au trone des conifères est un excitant du sysnerveux, et porte particulièrement son action les membranes muqueuses. A la dose d'un ime élevée progressivement jusqu'à vingt au in, administrée avec du miel ou autrement, est presque toujours très-utile dans les catarchroniques du poumon ou de la vessie, la diaratonique, c'est-à-dire causée ou entretenue par lâchement des tissus et le manque de vitalité, coulements muqueux, les névralgies, le rhusme chronique. Son principe actif réside dans ence de térébenthine que l'on isole par distillaCelle-ci s'administre à doses graduées, depuis yues gouttes jusqu'à deux grammes, et semble er une action plus marquée sur le système rux. Elle triomphe souvent de sciatiques re3. Dans ces cas on peut élever la dose à huit mes par jour, pris dans du miel, en plusieurs sil le remède n'a pas produit d'effet au bout de huit jours, on doit y renoncer.

Al'extérieur, l'essence de térébenthine est trèscomme rubéfiante, et produit une révulsion dans la bronchite chronique, la coqueluche, évralgies superficielles, les rhumatismes mus. res.

goudron, produit impur et complexe de la dision du bois des conifères, jouit des propriétés térébenthine, et augmente plus qu'elle les sé- 
crétions urinaire et cutanée. On en prend de d à quatre grammes en pilules. Cependant son us en nature est peu avantageux. L'eau de goud c'est-à-dire qui a séjourné pendant quelque te. en contact avec cette substance, lui est justen préférée, surtout dans les maladies des bronch

La poix blanche ou poix de Bourgogne, en plâtre, usitée dans les affections pour lesquellei conseille la térébenthine, est d'un emploi gên: on la remplace avec avantage par des frictions $c$ sence. La poix noire est employée dans les can gnes pour faire mourir les vers intestinaux poulains.

La créosote produite par la distillation du dron n'est réellement utile qu'à l'extérieur. Dissi dans dix fois son volume d'alcool, on s'en sert: succès contre la carie, la gangrène, pour le pa ment des plaies et des ulcères de mauvaise nat et aussi pour arrêter les hémorrhagies peu gra une goutte fait cesser la douleur des dents gât

La distillation du goudron fournit aussi l'a phénique ou carbolique que l'on a cru capabl détruire les miasmes du choléra, mais qui n' qu'à l'état de solution concentrée et caustique. I ce cas, il détruit véritablement les virus.

Dans la pustule maligne et le charbon, après a fendu en croix la tumeur et cautérisé profondén au fer rouge, on fait avaler à l'animal (bœu h cheval) do huit à dix grammes d'acide phén dissous dans un litre d'eau, et l'on administr même dose en lavement. Ce traitement se ré deux ou trois fois par vingt-quatre heures pen trois jours; le quatrième, on n'administre qux ou deux doses. Pendant ce temps, l'animal $r$ 
nourriture fortifiante, boit de l'infusion de gen$e, d u$ vin, et on le frictionne avec de l'essence térébenthine. On trouvera ce traitement beauo plus certain que celui qui consiste en applica$s$ réitérées de feuilles de noyer contuses, sur la 3 débarrassée de son épiderme, sans préjudice régime tonique. Des expériences récentes ten- à prouver qu'il y a une variété de pustule sans gnité réelle, sans virus contagieux, que l'on -it par simple incision suivie de l'emploi des llients. Peut-être les cas nombreux de guérison les feuilles de noyer rapportés par des médecins, irtenaient-ils à cette variété. Toutefois, la quesn'est pas encore résolue, et dans le doute, on sitera pas à choisir l'acide phénique.

en que notre promenade ait duré déjà plus que bitude, je ne la terminerai pas sans vous donquelques notions intéressantes sur les propriétés SaULE BLANG (Salix alba), qui sert de type à la Ile des Salicacées, comprenant le Peuplier, le e pleureur, le Saule herbacé, le Tremble. Dans 1) famille, les fleurs mâles et femelles sont sépaelles n'ont ni calice ni corolle. Les étamines l'une, l'ovaire de l'autre, sont placés à l'aisd'écailles formant des chatons touffus allon-

écorce du Saule est très-amère et astringente, emploie au tannage. Celle des rameaux de deux t)is ans, récoltée avant la floraison, est la plus ire. Elle contient beaucoup de salicine, subce que l'on fabrique en grand, comme succéde la quinine, principe éminemment fébri(2), retiré du Quinquina. Il a fallu du temps à la 
médecine pour adopter ce toniquie et fébrifuge pulaire, d'un usage général dans les campagr Aujourd'hui il est officiellement reconnu que malades qu'il a guéris par milliers n'ont pas victimes d'une illusion.

On administre avant l'accès de 10 à 30 gramı

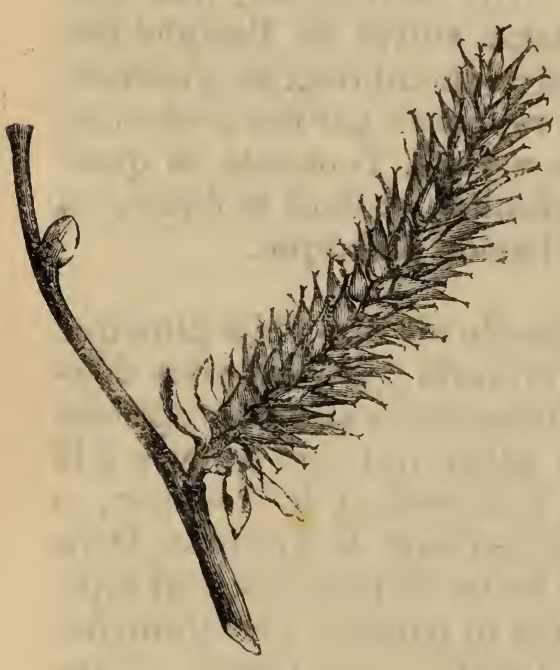

Chaton femelle de Saule.

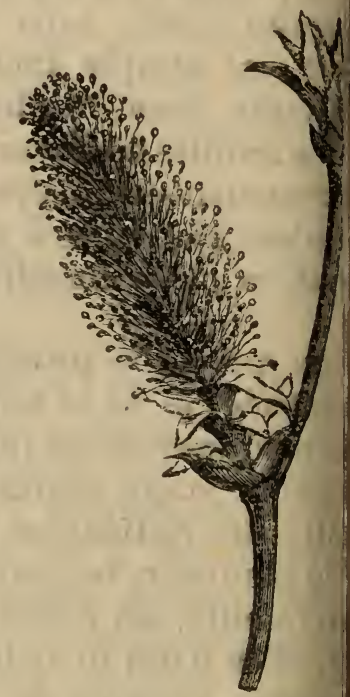

Chaton mâle de Saule

de l'écorce en poudre, ou bien une décoction 60 grammes dans un demi-litre d'eau.

Maintenant, deux mots sur les fièvres. Il y ef deux grandes classes : les unes revenant à int valles réguliers, ou intermittentes; les autres c. tinues. Celles-ci réclament des émollients, des acic pour calmer l'inflammation, un vomitif ou un $p$ gatif pour produire une dérivation sur l'appa 
quinine, qui en dérive. Mais il ne guérit pas jours, et l'on est souvent obligé de lui associer astringents et des toniques. Son grand défau de coûter fort cher, et c'est ce qui a fait cherch le remplacer par des végétaux indigènes, parmi quels le Saule figure au premier rang. Puis vi nent les plantes qui contiennent à la fois un $\mathrm{p}$ cipe amer et du tannin, comme le Chêne, le in ronnier, et tant d'autres que nous avons étud Mais comment expliquer qu'un tonique amer comme la Gentiane, fasse disparaitre la fi intermittente? Simplement parce que la nature à guérir, et n'a souvent besoin que d'un peu d'ce un peu plus de force, pour mettre le mal à la pie pourvu que le malade ne vive pas continueller au milieu des circonstances qui ont altéré sa sa

Bien plus, les fièvres intermittentes chronic semblent, dans bien des cas, ne continuer que suite d'une habitude des organes soumis à fluence nerveuse. Alors, non-seulement les toni amers, les astringents, les antispasmodiques merveille, mais tout ébranlement violent du syst nerveux, toute réaction vive des organes, un très-froid, un purgatif ou un vomitif énergiq un excès de table, l'ivresse, suffiront pour ron l'habitude. La foi vive en un remède peut être as puisqu'il ne manque pas de gens guéris au mi de toiles d'araignée!

Profitons de ce beau soleil printanier pour aujourd'hui une bonne promenade et une al moisson de plantes. L'hiver cherche en vain à lt 
ntre les brises attiédies qui devancentle printemps. s prés sont verts, les arbres bourgeonnent, queles-uns déjà couvrent leurs rameaux de fleurs rfumées. La terre semble pressée de nous offrir dons. Les germes brisent leur enveloppe, la e monte : c'est le grand réveil de la nature. ons la voir de près, admirer ses merveilles, et demander quelques-unes de ces vérités, que l'on elle à tort des secrets puisqu'il suffit de l'interser pour les apprendre.

irrêtons-nous un instant devant cette plate-bande; us allons prendre sur le fait le phénomène capi-
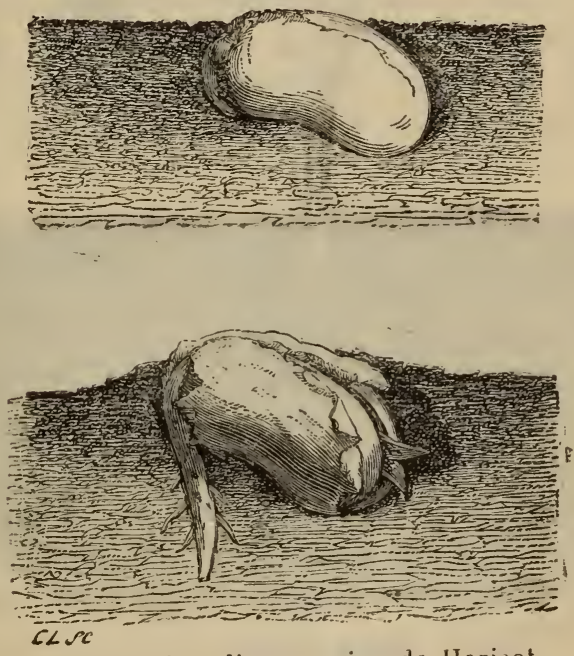

Germination d'une graine de Harivot.

de la vie des plantes : la germination. Voyez e graine de Haricot que je viens de déterrer. flfée par l'humidité, elle a brisée son enveloppe, t'on voit paraître d'un côté un filament charnu, 
fragile, d'où partent quelques petits filets; c'esta racine qui s'enfonce dans le sol. A l'autre ext. mité, s'ouvrant passage entre les deux moitiés dia graine, se montre la pointe de deux petites feui

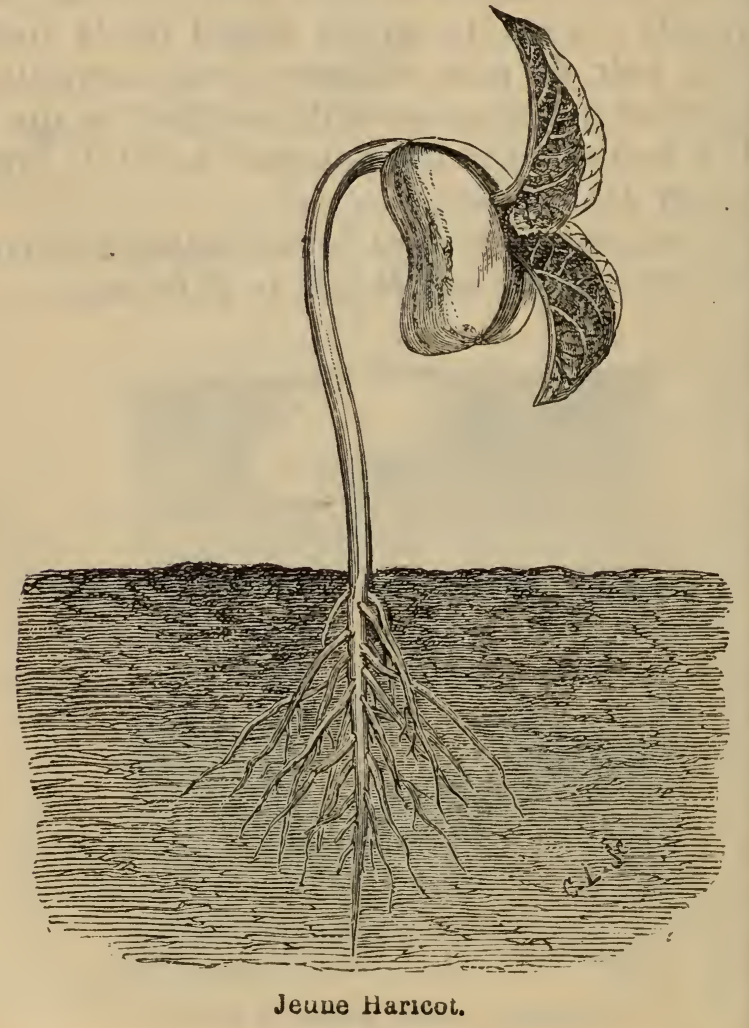

plissées comme les ailes d'un papillon nouveauLe germe de la plante, l'embryon, caché dan graine s'ouvrant, sous l'influence de la chaleus de l'humidité, laisse voir quatre parties bien 
ctes : les deux moitiés de la graine, nommées uilles germinatives, ou cotylédons; le bouron, ou gemmule; un commencement de tige, tigelle, et la radicule qui deviendra la ra10.

Les graines de quelques plantes, celles du Blé, r exemple, n'ont qu'un cotylédon. D'autres en it complétement privées, comme nous aurons zasion de le voir.

Jes enveloppes de la graine ne sont pas seulement stinées à protéger l'embryon; elles ont aussi pour e de nourrir la jeune plante en attendant qu'elle $t$ assez forte pour puiser dans le sol et dans l'atsphère les éléments nécessaires à son accroisseint. Aussi trouvons-nous, dans ce sujet, âgé à de quelques jours, les cotylédons flétris et lisés.

e vois avec plaisir, mes amis, que vous vous inessez à ces notions élémentaires, et que vous ne is laissez pas effaroucher par quelques mots un grecs ou latins; c'est la preuve que vous pre: goût à nos causeries. Continuons ainsi, et tout nous promenant, tout en recueillant des plantes, remêlant la pratique et la théorie, nous apprenins peu à peu, sans efforts, les éléments de la anique. Maintenant, aux champs, cueillez de ite et de gauche, et dans votre moisson je ferai shoix des plantes qu'il vous importe le plus de Inaître.

I tout seigneur tout honneur. Saluons la Vioe, qui se montre timidement entre les feuilles, umière fleur, premier parfum, emblème justent cher aux poëtes. 
La Violette odorante sert de type à la famille di Violarinèes, à laquelle appartient aussi la Pensé Les feuilles sont radicales, cordiformes, c'est-i dire échancrées en cœur, soutenues par un lon

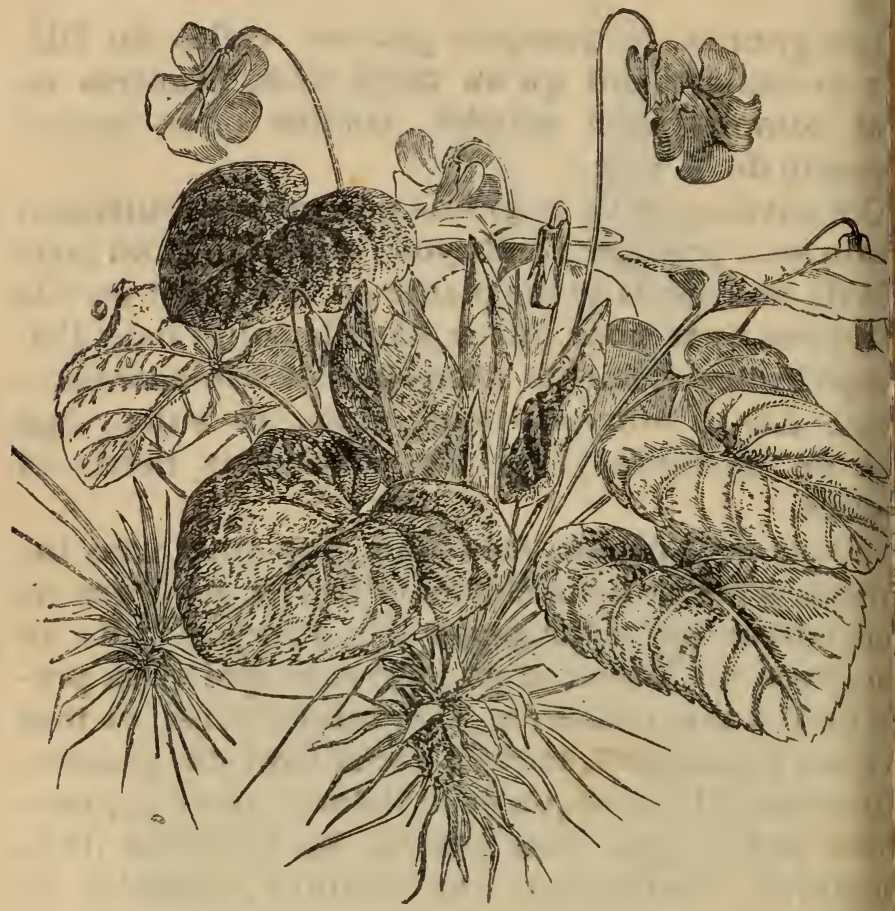

Violette.

pétiole, et légèrement duveteuses. Les fleurs, radi cales comme les feuilles, sont portées par un lon pédoncule grêle qui se recourbe au sommet. De cinq pétales de la corolle, le supérieur, plus gran que les autres, se termine en éperon à sa base. Toutes les parties de la plante sont utiles. Le 
illes fraîches forment un cataplasme émollient. ur suc, à la dose de deux onces, constitue un atif doux. Les fleurs au parfum à la fois suave rès-diffusible, sont émollientes, légèrement diarétiques, et, comme telles, d'un emploi journaen infusion théiforme, à la dose de 4 à 10 mmes par litre d'eau, dans les bronchites, les irrhes, les fièvres éruptives. Fraîches, elles sont jeu laxatives. La racine possède une propriété voive à peu près analogue à celle de l'I pécacuanhá, eut très-bien le remplacer dans les cas où ce fier semble indiqué, soit à dose nauséeuse, soit sse vomitive, surtout chez les enfants et les pernès délicates. Ellé peut rendre aussi de grands ices dans le catarrhe pulmonaire chronique, la ieluche, la dyssenterie. La dose vómitive et grative pour un adulte est de huit à douze nmes de poudre de racine ou de raciné coupée menu, en décoction dans un verre d'eau, pris eux fois. Si l'on recherche surtout l'effet voif, il vaut mieux administrer de deux à quatre - nmes de la poudre récente dans de l'eau sucrée. i Violette SAUVAge, Violette inodore ou Viode chien (Viola canina) est sans odeur. Les riétés de sa racine sont à peu près les mêmes celles de la Violette odorante, mais ne paraissent d'un effet aussi certain.

jus savez que la culture a produit des variétés rirquables de cette fleur, entre autres la Vio13 de Pañme, et celle dite remontante, qui donne iseconde floraison en automne. Sa culture dede peu de soins, on la multiplie par semis, par is de pieds; elle prospère surtout dans une re terre, à mi-ombre. 
Voici une plante amie de l'homme, vulgaire, partant peu appréciée dans les villes, où l'on ai ce qui est rare, ce qui vient de loin et se paye ch. Mais pour nous qui cherchons à combattre ces tendance, en vulgarisant la connaissance des chesses naturelles que nous offrent les champs, vulgarité ne peut être qu'une bonne recommanc tion. Vous la connaissez bien, cette plante; $\epsilon$ croît partout : dans les prairies, au bord des cl. mins, les chèvres, les moutons et les vaches la cherchent malgré son amertume, et vous vous ê souvent amusés à disperser d'un souffle vigoure ses fruits à longues aigrettes. C'est le Prssen (Leontodon taraxacum), nommé aussi Flor d'or, Dent de lion.

La racine, vous voyez, est grosse comme le doi d'un brun rougeâtre en dehors, blanche en deda Elle possède les mêmes propriétés que les feuill longues et profondément découpées, mais c] nous ce sont surtout celles-ci qu'on emploie. L amertume franche n'a rien de désagréable, av les mange-t-on en salade au printemps. Plus t: elles deviennent coriaces et contiennent plus principe actif.

La décoction de pissenlit, à la dose de $3 C$ 60 grammes de feuilles fraîches, leur suc à la $\mathrm{d}$ de 50 à 150 grammes, agissent comme antiscor] tiques, toniques, diurétiques et dépuratifs. L eniploi prolongé est utile dans les affections ch niques de la peau, la débilité des organes digesti efficace dans les engorgements du foie et de la $r$ qui accompagnent si souvent les fièvres de mar: ainsi que dans les maladies bilieuses. Nous en u rons largement, en dépit de la mode, et soyez s 
ue nous nous en trouverons bien. Il est toujours cile de se procurer la plante fraîche, cependant

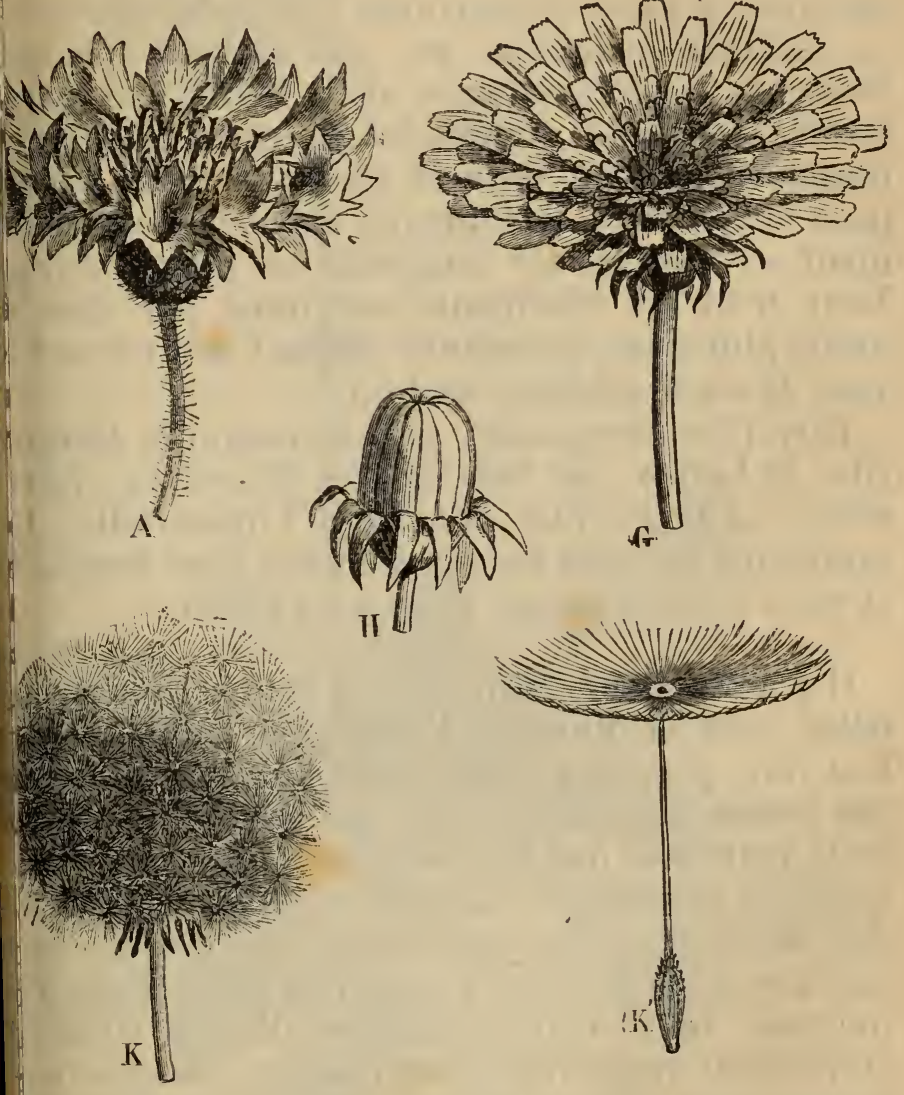

Afleur de Bleuet. - Pissenlit. G, capitule en fleurs. - H, le ième avant son épanouissement. - $\mathrm{K}$, ensemble des fruits. , un de ces fruits isolés.

peut récolter la racine au milieu de l'été pour aire sécher. 
La fleur de Pissenlit mérite de fixer un insta. votre attention. Elle est de la famille des Composé ou Synanthérées, l'une des plus nombreuses, r marquable par la disposition de ses fleurs réuni. en capitule et insérées sur une sorte de platea Dans ces agglomérations, dont l'ensemble pars former une fleur unique, les fleurons sont tant complets, comme àans le Bleuet, tantôt, comn dans le Pissenlit, la Chicorée, ils consistent seul ment en une étroite languette ou demi-fleuro: 'Leur fruit est d'ordinaire couronné par une a grette plumeuse, parachute élégant qui permet \& vent de les disséminer au loin.

Parmi les composées, je vous citerai la Margu rite, la Laitue, les Salsifis, les Chardons, l'Art chaut, la Camomille, l'Arnica, l'Immortelle. L 'caractères de cette famille sont des plus frappant et vous n'aurez pas de peine à les retenir.

Il y a des fleurs dont le nom seul est un poëm telles sont la Violette, l'Aubépine, la Primevèr Les voir, y penser même, c'est rappeler à l'instaı des scènes du premier âge, des heures délicieuse de la jeunesse, des impressions toujours vives toujours heureuses. Enfants, nous avons tous fa des bouquets, des guirlandes avec ces Coucou dorés que mars sème le long des haies et dans le prairies. Je vois que plusieurs d'entre vous or amplement renouvelé connaissance avec ces fleu favorites. Je vais en profiter pour vous dire à qui elles sont utiles.

La Prinevìne (Primula officinalis) est lo typ de la famille des Primulacées.

C'est une plante vivace dont une longue cultur 


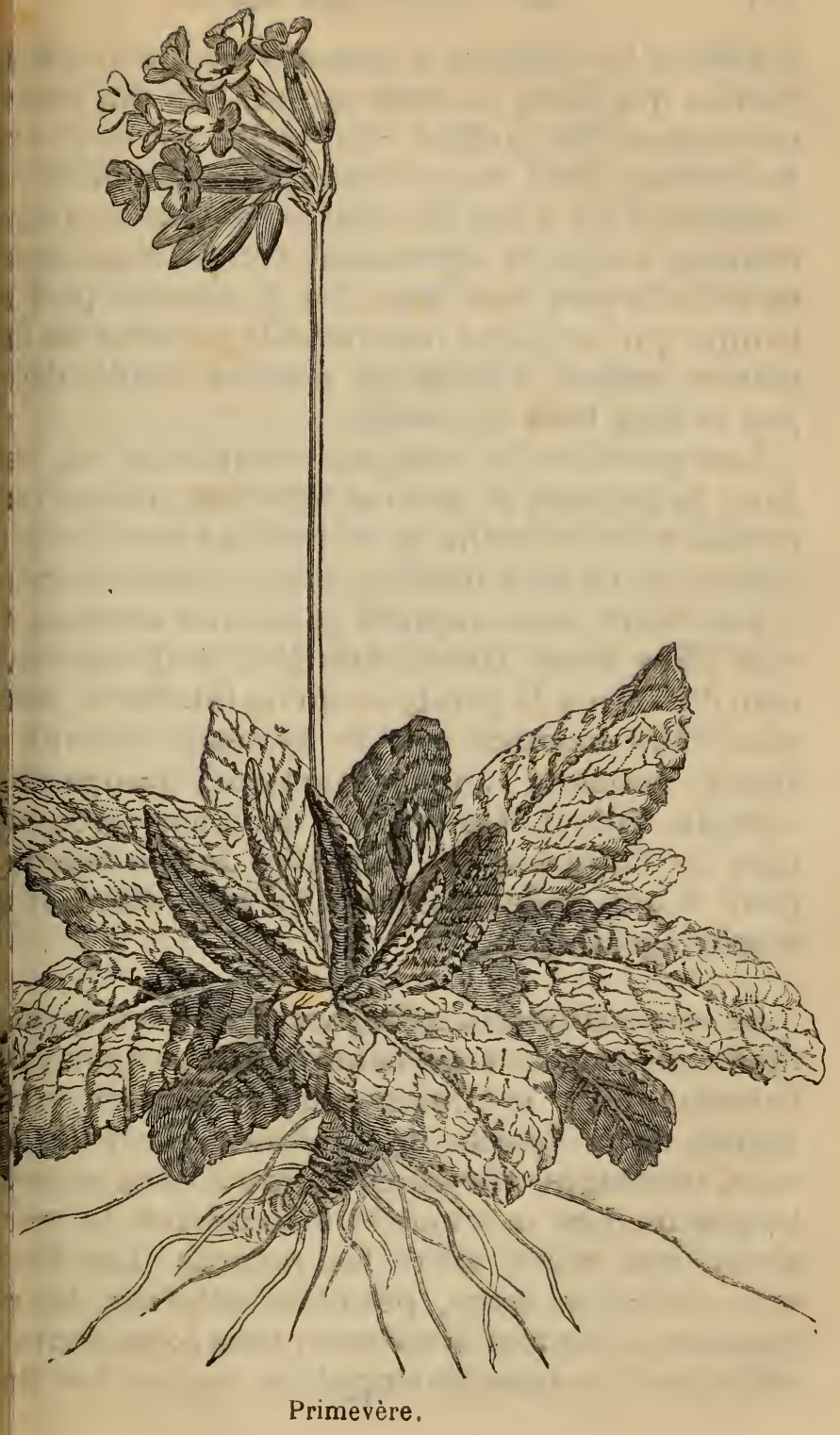


a obtenu les variétés à grandes fleurs de cou variées qui font, en cette saison, l'un des pren ornements des jardins. La racine est une so horizontale d'un rouge brun, garnie de fibres ? châtres. Il n'y a pas de tige, les feuilles oblon̨ dentées, ridées et légèrement velues, s'amincis en pétiole vers leur base. De la souche part hampe qui supporte une ombelle penchée de fl jaunes, tachetées d'orangé, dont la corolle dép peu le long tube du calice.

Les gens de la campagne emploient sa ra dans la gravelle et comme fébrifuge, mais c'es remède très-incertain, et en tout cas peu énergi auquel on ne doit attacher aucune importance.

Les fleurs ne manquent pas d'une certaine : vité. Elles n'ont jamais mérité à la Primevèr nom d'Herbo à la paralysie qu'on lui donne par: mais elles exercent sur le système nerveux action calmante, antispasmodique, comparab celle du Tilleul. On pourra donc user avec as tage de l'infusion en vase clos de ces fleurs, joint à une belle couleur d'or une odeur et saveur agréables.

Le sous-arbrisseau grimpant que vous voyez rouler ses rameaux sarmenteux aux arbustes di buisson, c'est la Douce-Amère (Solanum dui mara), de la famille des Solanées. Dans les li frais, ombragés, elie atteint jusqu à deux mètres: longueur. Les moutons et les chèvres la rech. chent, son odeur attire les renards. Les feuis sont ovales, en cœur, pétiolées, alternes, les us entières, les autres divisées en trois lobes dont dis petits et l'un très-développé au centre. Les fle 
ettes ou blanches, assez semblables à celles do Morelle, disposées en corymbes un peu irréguis vers le sommet des tiges, durent de juin à tembre, et produisent des baies arrondies, cges, accompagnées du calice de la fleur. On lui onné les noms de Vigne de Judée, Vigne saue, Morelle grimpante, Herbe à la fièvre, Loque, ve-chien.

es tiges d'un an ou deux sont la partie usitée. les récolte avant l'apparition des feuilles ou à n de l'été : au bout d'une année elles commen\% à perdre une partie de leurs propriétés.

a meilleure préparation est la décoction des raux coupés et fendus, dans la proportion de $\mathbf{1 5}$ grammes graduellement augmentée jusqu'à t 90 grammes par litre d'eau à prendre en vingtitre heures.

a Douce-amère est stimulante, sudorifique, déative et faiblement narcotique. Son action varie n les constitutions et les désordres apportés s l'économie par la maladie, mais d'ordinaire agit directement sur le tube digestif et secon'ement sur le système nerveux. A haute dose, cause des vomissements et des évacuations tndantes, provoque la sueur, augmente la sécréc. de l'urine; puis viennent des crampes, des Irdissements, des vertiges.

près avoir joui d'une haute réputation, la Doucere est tombée dans un oubli aussi injuste que Houanges des anciens auteurs étaient exagérées, st ainsi que la médecine abandonne souvent des nts actifs, et d'une utilité incontestable.

ans nier absolument les avantages qu'on a pu rbtenir dans l'hydropisie et les scrofules, il vaut 
mieux combattre ces maladies par des remèdes I certains, et réserver notre Solanée pour quelq cas de rhumatisme chronique, les catarrhes in térés, la zoqueluche, les ulcères indolents, les d leurs dans les os ou leur enveloppe, et principe ment pour les dartres et autres maladies rebe de la peau. Mais un point important, c'est de c ner des doses suffisantes, jusqu'à produire mê quelques accidents légers, maux de tête, étourc sements, et de continuer la médication pendant temps assez long, non-seulement pour dissiper symptômes actuels, mais pour combattre la dis! sition morbide ou le principe même de la malad Que d'insuccès on n'aurait pas à enregistrer, si 1 administrait toujours des remèdes bien choisis bien conservẻs, préparés avec soin, et contint avec persévérance!

Les feuilles de Douce-amère offrent à un tri faible degré les propriétés calmantes des feuilles Morelle et des autres Solanées, mais elles so émollientes, et, comme telles, peuvent être el ployées en cataplasmes sur les tumeurs simples les contusions.

La Douce-amère se multiplie facilement, $p$ graines, par boutures, marcottes ou éclats du pie On en garnit les tonnelles, les murailles, qu'e. décore, pendant toute la belle saison, pár śes gra pes de fleurs violettes et de fruits rouges.

Lo Nargisse des prés (Nárcissus psêudona cissus) vous est aussi familier que la Primevèr Comme elle, il se montre de bonne heure dans prés et les bois. Ii est de la famille des Amaryll dées, qui compte parmi ses membres l'odoran 
Tbéreuse. Il est connu sous les noms de Narcisse vage, Faux-Narcisse, Aiault, Porion, Jeannette, chette des bois.

'est une plante bulbeuse dont l'ognon lisse, ns comme le poudedonne naissance ine tige haute l'iviron 30 centires, remarquaIpar ses deux an. Is saillants, enorée de cinq à six lles moins hauqu'elle, lisses et lngées en forme iéc. Avant leur eloppement, les irs sont renferis dans une en()ppe ou spathe se fend et pero sur la tige. Ces $r$ d'un jaune 3. penchées sur hampe, présentrès-distincts caractères de la ille dont elles t le type. 'enveloppe des

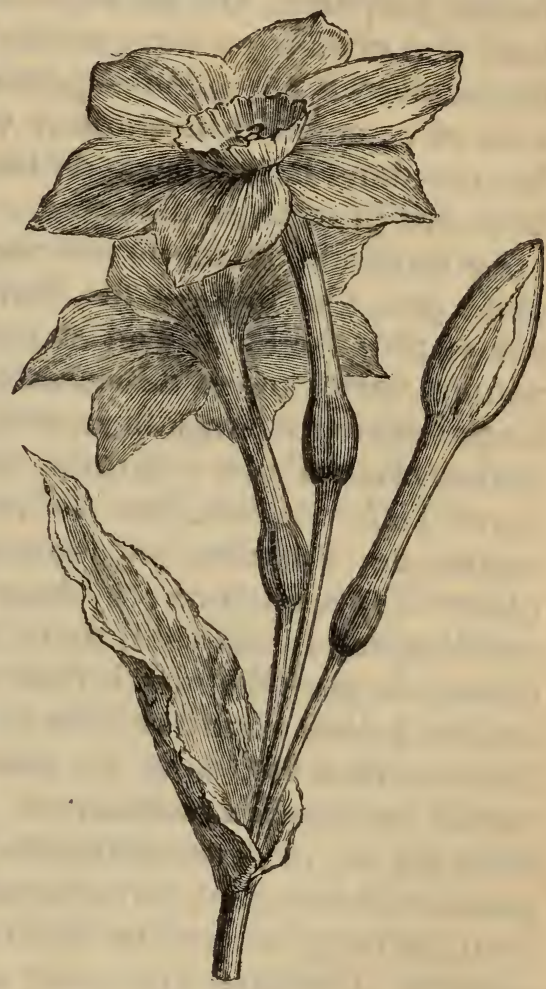

Fleurs de Narcísse cultivé,

anes reproducteurs, ou enveloppe florale, forme long tube, étroit et simple dans sa partie infére, mais qui s'élargit et se dédouble de mare à former deux limbes, l'extérieur divisé en 
six languettes lancéolées, l'intérieur d'un jaune formant une couronne un peu évasée.

On peut récolter en tout temps les bulberd Narcisse. Quant aux fleurs, il y a deux mani de les préparer. On les sèche rapidement dans four peu chaud, ou bien on les expose à l'humit de manière à ce qu'elles perdent lentement lif sucs et prennent une couleur verdâtre. A cette férence dans la manière de les sécher corress? dent des propriétés diverses.

Le bulbe de Narcisse est vomitif, mais on l' ploie peu. On préfère les fleurs, d'un usage sûr et plus facile, et l'on choisit celles qui sont venues vertes par la dessiccation lente. L'eau bo lante développe singulièrement leurs propriétés la décoction d'une vingtaine de fleurs produit 1 jourş? T'effet désiré. Ces fleurs agissent comm racine de Violette, et peuvent comme elle $r$ placer l'Ipécacuanha, à dose nauséeuse, surl chez les sujets délicats, dans les catarrhes pul, naires, la période non inflammatoire de la coc luche; à dose vomitive dans le début inflammat de cette maladie, dans les diarrhées chronique: même quelques dyssenteries. Quant à son ut dans les fièvres intermittentes due en partie à pouvoir légèrement narcotique, elle est trop fa pour qu'on y ait recours dans les circonstances dinaires. Lorsque l'on veut utiliser les propri antispasmodiques et narcotiques des fleurs, il 1 choisir celles qui, séchées rapidement, ont conse leur couleur jaune.

Vous connaissez les variétés de Narcisses ol nues par la culture. Elles sont certainement $\mathrm{F}$ bdorantes que le Narcisse sauvage, mais celui 
t à l'élégance et à la grâce champêtres des quaqui nous feront lui accorder une place dans e collection.

our terminer notre promenade, je vais vous er du Pêcher, que nous voyons ouvrir ses rs précoces aux rayons déjà vivifiants du soleil nars.

is fleurs, d'un rose tendre, ont une odeur e, agréable et une saveur amère. Elles sont ement purgatives et anthelmirithiques. L'infudans du bouillon de veau ou dans du lait, à la d'une petite poignée de fleurs par 500 gramde liquide, convient très-bien aux enfants, il faut l'administrer par portions de demiio en demi-heure, jusqu'à ce que l'action du de commence à se faire sentir.

Is feuilles et la seconde écorce sont purgatives brifuges, anthelminthiques et diurétiques. La cition de 30 grammes de feuilles fraîches dans Oprammes d'eau purge aussi bien que le Séné. linistrée entre les accès d'une fièvre intermitelle opère en peu de jours la guérison. Il havoir soin dans ce cas de graduer les doses lanière à n'obtenir qu'une légère purgation. conde écorce s'emploie de la même manière. Cites les parties du Pêcher contiennent, en ité très-minime, un poison violent, l'acide ique, qui leur donne une vertu sédative. Un a asme des feuilles pilées calme la douleur des tis enflammées, des ulcères, des contusions.

PẾGher (Amygdalus persica), de la famille osacées, nous est venu de la Perse. On le lolie par semis, et surtout par greffe, sur l'A- 


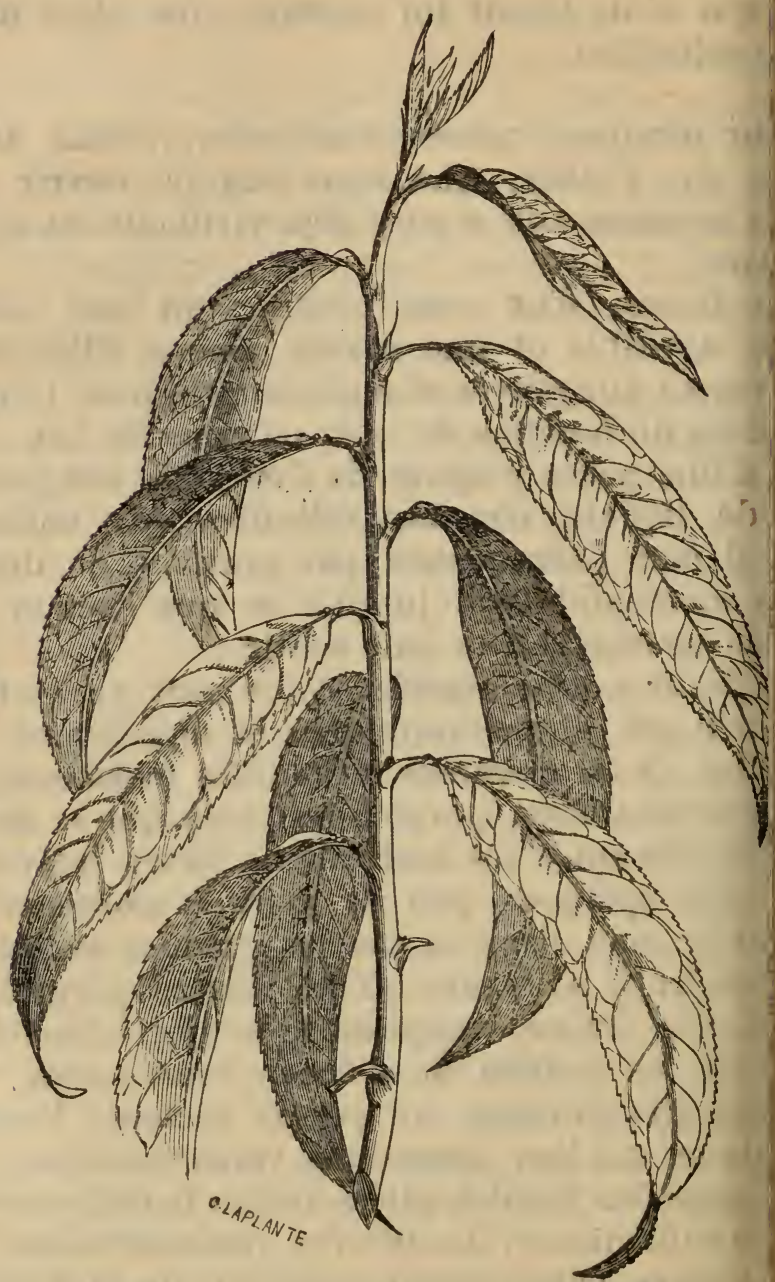

Rameau de Pècher.

fonde et substantielle, souvent remuée et fu 
ne bonne exposition, et un abri mobile contre les elées tardives qui brûleraient ses fleurs. Sa culire est toute une scienco, mais aussi quels surpreants résultats! Quelle distance entre le fruit petit, erdâtre et sec de la Perse et les magnifiques êches de Montreuil! Qu'a-t-il fallu pour obtenir changement? Un travail intelligent, une culture isonnée et continue; je pourrais dire, la volonté. ravail, étude, persévérance, bon propos, tels sont, i ce monde, les éléments du progrès. Si l'homme t capable d'améliorer, de transformer des fleurs, is fruits, des arbres, des animaux même, il peut, yez-en sûrs, apporter dans l'ordre moral des rfectionnements non moins remarquables, et par fraternité, l'honnéteté, le travail, réaliser le proème de la vio heureuse.

Cet Agaric que nous venons de cueillir appare int à la nombreuse famille des Champignons : jn profite pour vous donner quelques détails sur (; plantes qui fournissent, selon l'espèce, un mets szculent ou un poison mortel. Et d'abord, je vais sis doute vous étonner en vous disant que la p.nte du champignon ordinaire croît sous terre, equo la partie qui nous est familière sous ce nom cistitue seulement le réceptacle des organes repiducteurs. Quant à la plante elle-même, ce sont t.t simplement des filaments blanchâtres, entreisés, feutrés, nommés en botanique mycelium vulgairement blanc de champigroon. Mais il y es Champignons dont la plante et les organes roducteurs vivent à ciel ouvert, par exemplo 
ceux qui causent la maladie de la Vigne, et d'a. tres qui ne paraissent jamais à la surface du s, comme les Truffes.

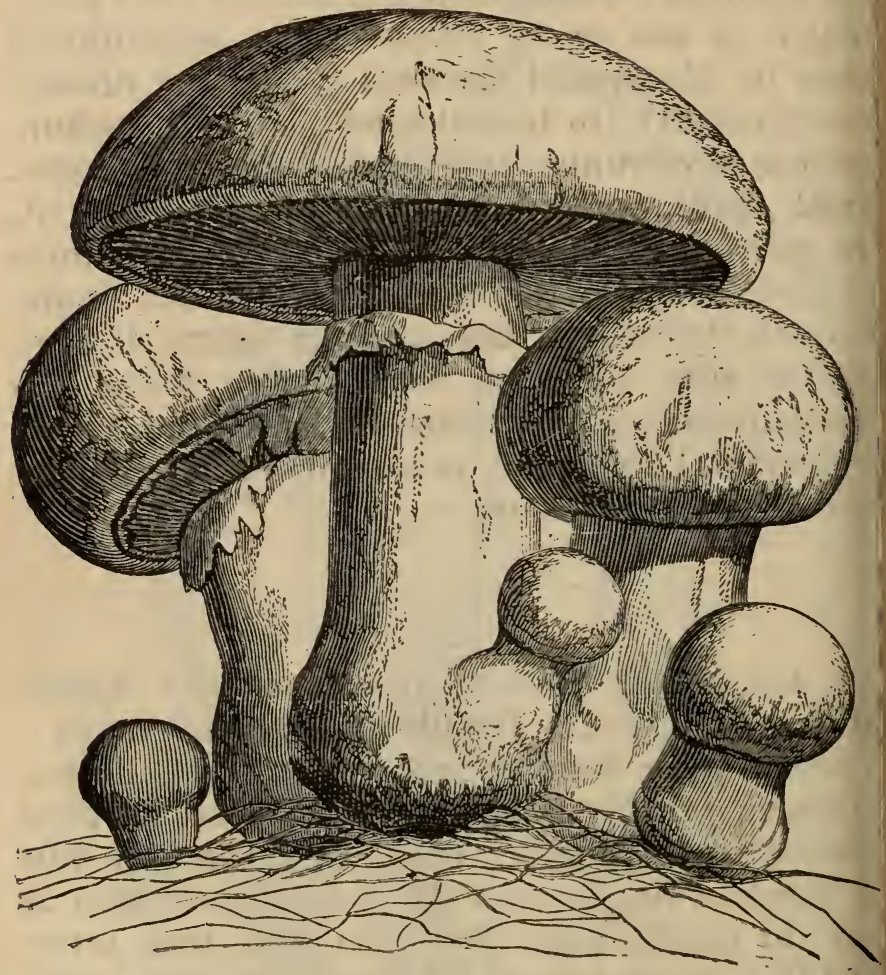

Champignons.

Si nous prenons pour exemple l'Agarig GHampi TRE, vulgairement appelé Agaric comestible o Champignon de couche, je vais pouvoir vous don ner une idée de la naturo do ces végétaux. Le pie ou stipe est surmonté d'un chapeau lisse, blanc or 
jaune pâle qui s'étale peu à peu et laisse voir en dessous des feuillets rosés qui passent bientôt au brun. Les feuillets sont pendant quelque temps recouverts par une membrane qui se déchire et orme un collier autour du stipe. Si l'on prend sur 'un de ces feuillets une tranche mince et qu'on 'examine au microscope, on voit que la surface veloutée au toucher est formée de cellules sailantes dont quelques-unes portent quatre pointes erminées par un petit sac qui constitue une spore, 'est-à-dire l'organe reproducteur capable de gerner et de reproduire du mycelium qui, à son tour, lonnera naissance à des Champignons. Les organes e la reproduction se trouvant ainsi cachés, et ne e révélant qu'à un examen attentif, on a donné ux plantes qui présentent cette parcularité le nom e Cryptogames, par opposition aux plantes Phaérogames qui exposent aux regards leurs fleurs lus ou moins compliquées.

Je n'ai pas à vous enseigner ici quels sont les rampignons comestibles, mais je puis vous donner mme règle générale de ne jamais cueillir ceux di poussent dans les endroits très-humides, dont chapeau est visqueux, couvert de verrues, qui - se pèlent pas facilement ou dont le stipe est nflé à la base. Une saveur âcre et acide, une leur nauséabonde, une chair molasse qui noircit contact de l'air, sont aussi caractéristiques des 'pèces vénéneuses.

Mais il y a des champignons dangereux qui resimblent beaucoup aux espèces comestibles. Pour (iter toute erreur on peut employer le moyen lin simple que voici : on fait cuire avec les chamljnons la moitié d'un oignon blanc; s'il devient 
bleuâtre ou brunâtre, c'est le signe qu'il s'est glis quelque intrus dans la cueillette. Il semble désc mais prouvé que l'on peut manger sans crain presque toutes les espèces vénéneuses après I aroir débarrassées des principes toxiques. Po cela on les coupe par morceaux et on les fait $\mathrm{m}$. cérer pendant quelques heures dans de l'eau for ment vinaigrée; on les lave ensuite à grande e: et on les blanchit par quelques bouillons.

Puisque nous avons aujourd'hui l'occasion nous occuper des plantes Cryptogames, je vais profiter pour vous parler de la Fougère dite Fo GÈne MALE (Polypodium filix mas) qui vous très-familière et dont l'étude, pourtant, vous $\mathrm{m}$ nage plus d'une surprise.

Vous avez remarqué sans doute que la partie i férieure des feuilles est comme brodée de points de lignes brunes. Ce sont les réceptacles des spor ou organes reproducteurs. Si nous suivons, da la terre humide, le développement d'un de c germes, nous verrons qu'il donnera naissance à u petite plante qui ne ressemble en rien à une fo gère. Cette plante est un être de transition, e n'est point destinée à vivre et à se reproduire; e fournit seulement deux organes séparés de repr duction qui, par leur action réciproque, sont pables de former une plante nouvelle qui sera $u$ fougère. Ainsi nous assistons à ce phénomène si gulier : une spore de Fougère se développe so forme d'une petite plante qu'on appelle protha qui porte des organes sexuels capables de repr duire non pas un autre prothalle, mais une fougè semblable à sa grand-mère. Ce mode de générati 
ui existe aussi chez quelques animaux inférieurs, appelle génération alternante.

Je n'ai guère besoin d'insister sur le port et l'asect de cette plante que vous avez si souvent occa-

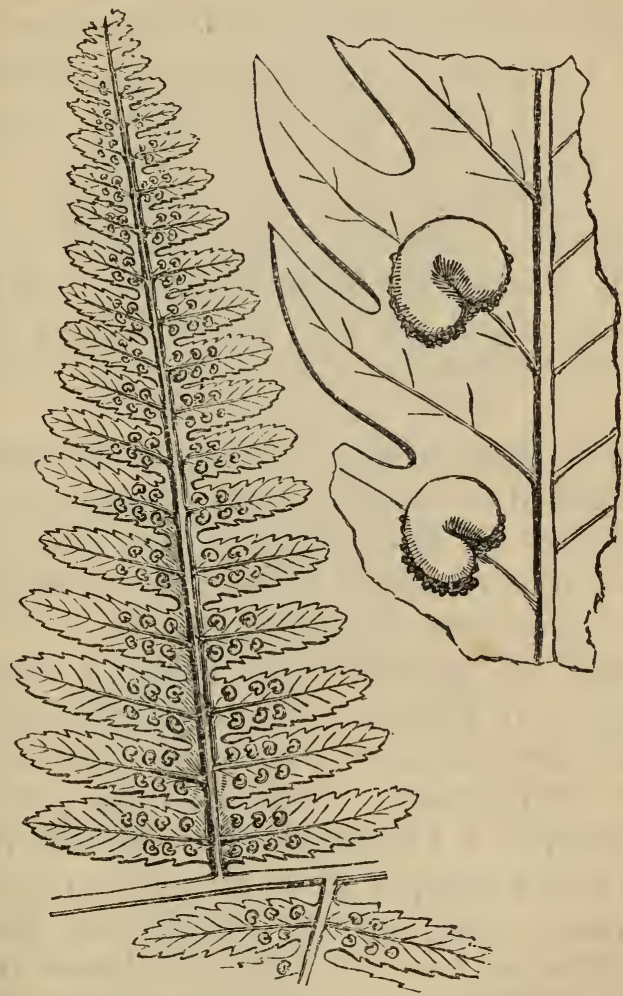

Feuille de Fougère mâle.

ih de voir; mais je veux attirer votre attention 1 lá partie souterraine ou rhizôme dont je viens (jueillir un spécimen. Comme vous voyez, il est la grosseur du pouce, noueux, écailleux, dc cou. 
leur brune à l'extérieur, blanchâtre à l'intérieus On peut s'en servir en tout temps à l'état frais, la dessiccation lui fait perdre une partie de ses pre priétés. Pour le commerce, on doit le récolter e été alors qu'il est dans toute sa vigueur et plein d sucs qui donnent une teinte verte à sa cassure. De gens peu scrupuleux mélangent aux rhizômes d Fougère mâle ceux d'autres espèces commune dans nos campagnes. C'est une pratique déshon nête et dangereuse, car il ne suffit pas que de plantes appartiennent à la même famille pour avoj les mêmes propriétés, et si l'on administre un r mède inerte dans un cas urgent, on s'expose perdre le seul temps propice pour une médicatio utile.

L'odeur de cette souche est un peu nauséeuse; I saveur, d'abord douceâtre, puis légèrement amèr et astringente, est due surtout à une huile volatil mélangée à une résine et à des corps gras qui con tituent les principes actifs de la plante.

Son usage, en médecine, est limité à l'expulsio du tænia ou ver solitaire. Je vous ai déjà indiqu les graines de citrouille comme un excellent tæn fuge, par conséquent nous n'avons pas besoi d'aller chercher à l'étranger des remèdes rares 0 d'un prix élevé lorsque nous en avons d'excellent sous la main, qui seront " prophètes dans leu pays toutes les fois qu'on les emploiera dans le conditions nécessaires au succès. Pour obtenir un guérison complète dans les cas de ver solitaire, ne suffit pas de faire expulser l'animal dans so entier, il faut s'assurer qu'il ne reste pas un autr ver dont la présence ramènerait tous les accident de la maladie. Il est donc prudent de continuer I 


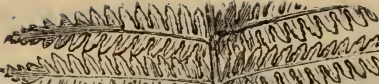

I

orit

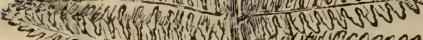

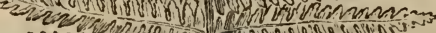

raphan on

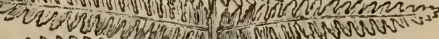

3 s.

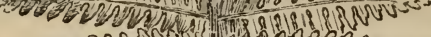

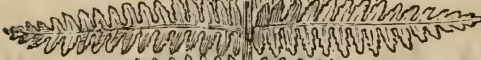

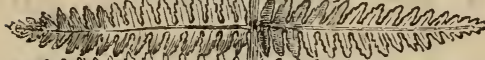

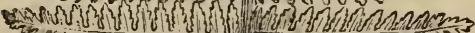

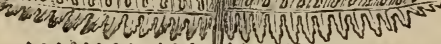

-32A An

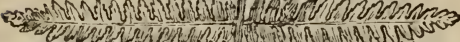

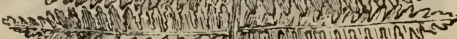

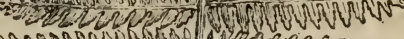

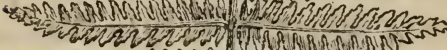

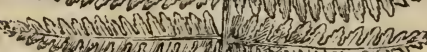

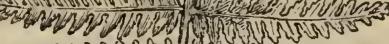

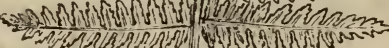

U)

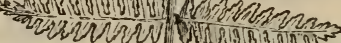

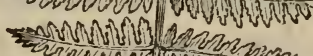

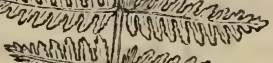

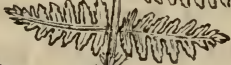

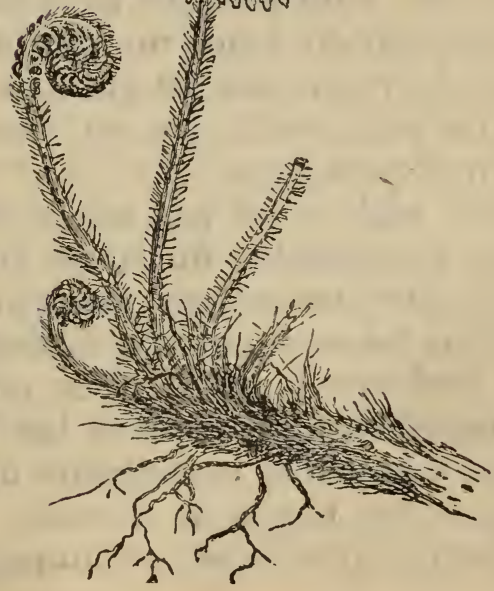

Fougère mâle. 
médication pendant plusieurs mois. Il faut d'ai leurs être familier avec la structure de ces an maux pour s'assurer que la tête très petite n'est $p$ restée dans l'intestin, car elle suffit pour repr duire cet animal. Pour éviter les récidives, le plt sûr est d'administrer pendant un certain tem] le remède à des intervalles de quinze à vin jours.

On trouve dans les pharmacies plusieurs prép rations de Fougère mâle, principalement une huj éthérée et un extrait résineux, mais la poudre la décoction du rhizôme, soit frais soit conser clans de bonnes conditions, suffit toujours dans pratique. Pour la décoction, qui doit se faire en 1 vase clos, on emploie de 30 à 60 grammes de $r$ cine concassée pour un litre d'eau qu'on laisse $r$ duire de moitié. Mais la manière la plus simple, peut-être la plus efficace d'administrer ce médic ment, consiste à mêler la poudre de rhizôme à qua tité égale de miel. On en prend à jeun de 60 90 grammes par jour pendant trois jours, puis facilite l'expulsion du tænia au moyen d'une for dose d'huile de ricin, soit 60 grammes. Si le pr mier essai ne réussissait pas, on recommencer au bout de quelques jours.

La Fougère mâle n'est pas seulement employ en médecine. Les peuples du Nord, si ingénieux tirer parti de leurs ressources, mangent les jeun pousses comme les asperges. En Sibérie on emplo la racine à parfumer la bière. Chez nous, les sor ches engraissent bien les porcs et les feuilles tes dres, mêlées à la paille, constituent un assez bc fourrage pour les temps de disette. Les feuill sèches sont employées dans les campagnes pour 
uche des enfants, et les coussins ou matelas que $n$ en fait sont beaucoup plus sains que ceux de ime. Ils sont utiles surtout aux enfants scrofu$\mathrm{x}$ ou rachitiques.

Remarquez, mes amis, dans ce jardin, ces arbres xquels les cisailles et la serpe ont donné des mes capricieuses de colonnes d'arches, de niches; st un membre de la famille des Conifères qui is a déjà fourni pour sujets d'étude le Genévrier le Pin Silvestre.

'IF (Taxus baccata), livré à lui-même atteint une ateur d'environ dix mètres. La tige, couverte ne écorce raboteuse, soutient un très-grand nbre de rameaux souples garnis de feuilles pertantes, c'est-à-dire qui ne tombent point sous tion du froid, d'un vert sombre, linéaires, trèsprochées, et rangées, sur deux côtés opposés. i fleurs femelles ressemblent à une pomme de en miniature surmontée d'un petit cylindre ux formé d'écailles allongées; les fleurs mâles, n plus nombreuses, ont l'aspect d'un petit bourin verdâtre. Elles sont axillaires, c'est-à-dire ichées immédiatement au rameau. A mesure que 'ule grossit dans la fleur femelle, les écailles qui maient comme une gaîne, une arille à sa partie lírieure, deviennent charnues et recouvrent peu à le fruit.

let arbre croît spontanément dans les montagnes hl'Italie, de la Suisse et des départements mérinaux de la France. On le propage facilement de rcottes, mais les sujets venus de graines sont 
plus grands et plus durables. Les graines restit souvent trois années en terre avant de germer. If se plaît de préférence dans les bonnes terres fr. ches; il aime l'ombre, surtout dans sa jeunes aussi le voit-on prospérer surtout dans les vallis à l'exposition du nord.

On a répandu beaucoup d'idées erronées au sut

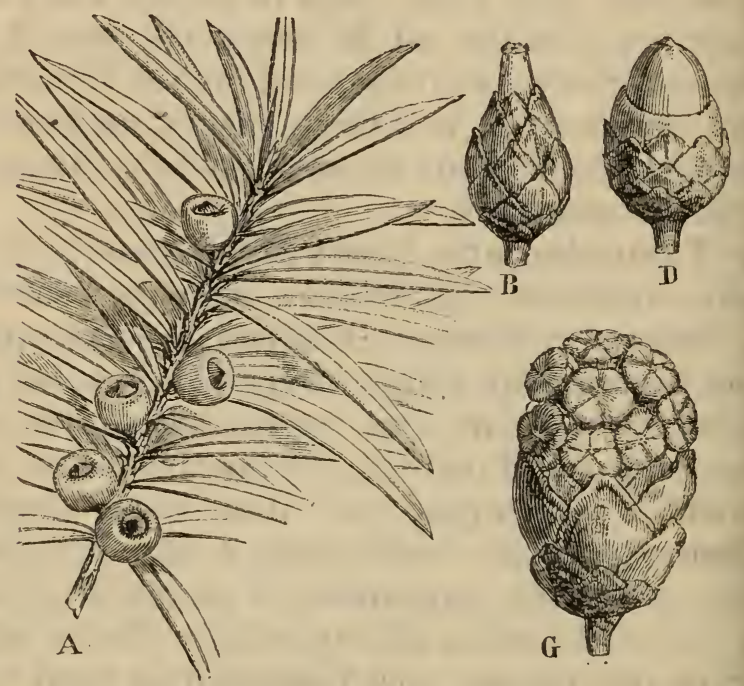

If. - A, rameau avec fruit. - B, fleur femelle. - D, fleur ferre plus avancée. - $G$, chaton de fleurs mâles.

de l'arbre qui nous occupe : on a prétendu son ombre même, comme celle du Mancenilli, pouvait donner la mort. Il est vrai que la plupt des gens peuvent séjourner impunément au mil d'ifs même fraîchement taillés, mais il n'en est $1^{\mathrm{s}}$ moins exact que chez certaines personnes, les énnations qui s'en dégagent peuvent, à la long, produire des accidents. Ses feuilles sont un pois 
ur les chevaux, les ânes, les moutons et les vaes, et il est à remarquer que ces animaux ne les ingent guère que pressés par la faim. L'étude de ses effets sur l'homme a démontré 'il agit d'abord comme poison irritant et âcre, jvoque des vomissements et des évacuations et ise l'inflammation de l'estomac; puis, lorsque l'abption commence à se faire, on remarque une acn narcotique et stupéfiante; inquiétude, éblouisnent, syncope ; les victimes tombent comme fouyées. Quant aux fruits, ils sont dépourvus de alités délótères, ils sont simplement laxatifs et ivent, comme tels, rendre quelques services. La oction des feuilles, administrée à petites doses, it on surveille avec soin les effets, a donné quelis bons résultats dans les affections rhumatisles. Quant aux propriétés emménagogues de cette nte, elles ne sont nullement prouvées tandis que a constaté des accidents mortels à la suite de usage. En somme, c'est un arbre qu'il faut contre plutôt pour s'en abstenir que pour essayer vertus curatives.

Iais s'il doit être rejeté de l'usage médical, l'If précieux pour l'industrie. Son bois, presque ourvu d'aubier, est d'un rouge orangé; le cœur ; à faire des ouvrages de marqueterie, de tour et énisterie. Son élasticité et sa durée le font rercher pour le charronnage; enfin la facilité avec ielle cet arbre se prête à la taille le faisait rercher autrefois pour certains ornements bizarres jardins heureusement passés de mode.

e passons pas devant ces touffes de Pervenches ' leur donner quelques moments d'atiention. Il 
est probable que cette plante doit plus à ses qu. lités extérieures qu'à ses vertus intrinsèques. $S_{1}$ feuillage toujours vert, le port gracieux de ses tiç sarmenteuses, l'étoile bleue ou blanche de ses flets qui parent, au mois de mai, les bois et les part ombreuses des jardins, en attirant sur elle une :tention sympathique, ont bien disposé les obseri. teurs à l'admettre au nombre des plantes bienf. santes. Autrefois les sorciers l'employaient da leurs philtres, d'où son nom de Violette des st. ciers, et en Flandre on en semait sous les pas (3 jeunes mariées d'une réputation intacte; en Itas on dépose encore des couronnes de pervenches : la tombe des jeunes filles. La vue de cette fle reportait Jean-Jacques Rousseau aux souvenirs (3 Charmettes, et Mme de Sévigné recommandait à fille a la bonne petite Pervenche "pour les ma de poitrine dont elle souffrait.

Tout en rendant hommage à cette favorite (3 jardins et des bois, nous ne pouvons pas, au po de vue qui nous occupe, lui accorder une place bi importante. La Pervenche (Vinca minor) appartict à la famille des Apocynées, dont nous n'auro guère à nous occuper. On l'appelle, en certais endroits, Herbe à la capucine. Les feuilles seus sont usitées en médecine. On les récolte d'ordina) avant la floraison, mais on peut les recueillir toute saison. Leur saveur, amère à l'état frais, $c$. vient astringente après la dessiccation. Elles cède facilement à l'eau leur principe amer et une qua. tité notable de tannin, ce qui la rend utile tou' les fois que l'on a besoin d'un astringent peu én gique.

Maintenant, arrachons une de ces touffes et ét. 


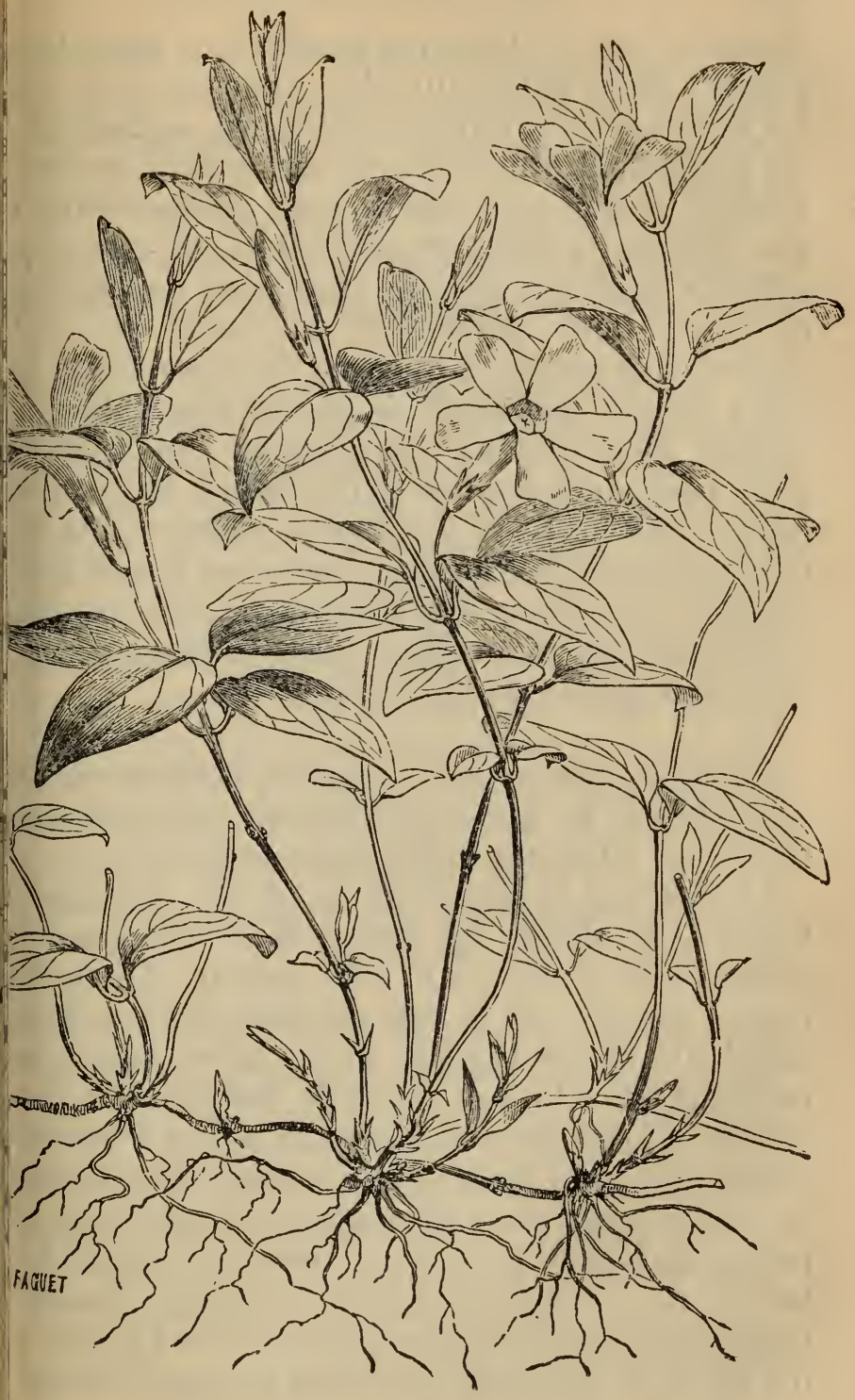

Pervenche. 
dions un peu la structure générale, le port de cte plante. Vous voyez que les rameaux qui porint les fleurs sont groupés de distance en distance ir un renflement qui forme souche et d'où partent 3 S racines, et que ces souches sont reliées entre eas par une partie assez grosse, solide, et que l'on per. rait, de prime-abord, prendre pour une racine couverte. Cette portion de la plante est la tie. Après avoir rampé à quelque distance sur la tee $\theta$ elle a donné naissance, à courts intervalles, à es nœuds d'où partent des rameaux qui se dressit pour porter des feuilles et des fleurs, et des racis qui s'enfoncent dans le sol pour procurer à la plaje un nouveau centre de vie. C'est ainsi que le Frais: produit des filets, des stolons origine de plars nouvelles. Le marcottage des jardiniers est te imitation de ce procédé naturel.

Puisque nous avons roccasion d'étudier les ti s des plantes, je vais vous donner, pour ce qui concerne, quelques indications générales.

La tige est la partie de la plante qui porte s feuilles, les fleurs, les fruits. Mais la tige peut $\hat{\epsilon}_{\theta}$ souterraine sans pour cela devoir être nomne racine comme on le fait souvent dans le lang:日 usuel. On donne le nom de rhizôme aux tiges rampent dans le sol donnant naissance à des meaux et à des racines. Lorsque la tige très-charr présente une forme arrondie, comme dans la pom? de terre, c'est un tubercule, si l'apparence esi peu près celle de l'ognnon, comme dans le Colchiq'. le Lis, on lui donne le nom de bulbe. Quant as tiges proprement dites, celles qui vivent à l'air lib, il y en a de dressées, de couchées, de rampant; 
ques-unes, comme celles du Liseron, du Houtrop faibles pour se tenir dressées, s'enroulent ur des troncs, des branches qui se trouvent leur voisinage, d'où leur nom de volubles ou biles; d'autres, comme les Pois, la Bryone, la 1e, se servent de vrilles pour s'accrocher et ter vers la lumière, ce sont des tiges grimtes.

rsqu'une tige, au lieu de grandir par la proion successive de rameaux, s'allonge seulement le sommet, comme nous le voyons dans les hiers, on l'appelle stipe; si la tige forme, de nce en distance, des nouds comme dans le , les Céréales, elle prend le nom de chaume eut être plein comme dans la Canne à sucre, -plein comme dans le Maïs ou fistuleux, c'este creux, comme dans les céréales. Enfin lorsque e est formée de plusieurs couches concentricomme nous l'avons vu en examinant la strucles bois, et se dédouble à partir d'une certaine ur en branches et en rameaux, elle constitue onc. On a classé, en outre, les tiges, par rapi leur durée, en annuelles, bisannuelles et es.

is avons dit qu'il ne fallait pas confondre une exouterraine avec une racine, et nous avons parmi les tiges le tubercule de la pomme de ce qui vous a fort étonnés. Mais si nous conns comme signes distinctifs de la tige la préde feuilles ou de cicatrices laissées par la ou l'avortement de ces organes, nous ne cour3lus risque de faire une confusion. Toutefois, la pomme de terre, la tige souterraine était etite au moment où elle portait des feuilles, 
et ces organes, à l'état rudimentaire, sont tombe laissant sur le tubercule qu'une cicatrice peu ble, il est vrai, mais que l'on ne trouve pas das racine du Dahlia.

Bien que notre promenade se soit prolongée 1 que de coutume, je veux encore vous parler ci plante qui vous est familière, la vigne, sur laq je vous donnerai quelques détails tout en $1 \mathrm{~g}$ gnant notre école, me bornant d'ailleurs, fau temps, aux applications médicales qu'elle est ceptible de recevoir.

La Vigne (Vitis vinifera) est le type de la fail de Ampelidées. Je n'insisterai pas sur son ports feuilles, ses vrilles, mais j'aurai occasion de faire remarquer ses fleurs, disposées en gr.1 composée et dans lesquelles les organes de rer duction sont très-distincts après la chute des pélle

Les feuilles de vigne sont astringentes, et con telles, utiles dans la dyssenterie, la diarrhée cr nique, les hémorrhagies passives, c'est-à-direr duites par un manque de tonicité, de vitalité des ties. Vous avez entendu peut-être les comnr vanter comme remède des ophthalmies la sève cr line qui découle, au printemps, des incisions aux rameaux, mais c'est une des nombreuses erju populaires que je m'applique à vous signaler pleurs de vigne sont tout à fait inertes.

Les raisins bien múrs sont nourrissants, r: chissants et un peu laxatifs, leur usage est vent utile, dans les maladies chroniques, aux sonnes d'un tempérament sanguin ou bilieux, i constitution sèche, irritable. Aussi heaucou malades se rendent-ils chaque année en $\mathrm{Bo}$ 
jour, en trois, quatre ou cinq fois, et autant c $\theta$ possible le malade cueille lui-même son repas ente promenant; de sorte qu'il faut tenir comptele l'influence salutaire des conditions hygiéniqs lans lesquelles il se trouve. Le raisin agit par propriétés alcalines, laxatives et diurétiques.

Le suc de raisin vert ou verjus est fort vlo comme tempérant dans les maladies inflammatoi s, les fièvres bilieuses, les irritations de l'estomaet des intestins, et quelquefois comme gargarize dans les angines, le ramollissement des gencives, On l'emploie comme boisson à la dose de 100 à 00 grammes par kilogramme d'eau

Quant au vin, il n'y a guère que re médecin ui puisse bien apprécier l'opportunité de son emloi dans les maladies et les doses convenables chaque cas. Il convient dans toutes les maladiebu la faiblesse n'est pas causée par une inflammatin On le donne avec avantage dans la période de j tration de la fièvre typhoïde et des affections crt gieuses, dans certaines pneumonies qui ne delı dent pas la saignée. Enfin, bien que ce mode ministration ne se soit pas généralisé, il ré: d'expériences bien faites que des lavements dev. de 150 à 200 grammes précédés d'un lavement tiède qui débarrasse l'intestin, produisent $d^{\prime} \in{ }^{\prime}$ lents résultats dans les maladies chroniques convalescence des maladies aiguës. Je n'ai vous parler ici des dangers de l'abus du vin, les connaissez sans doute; je vous dirai seulce que l'ivrognerie prolongée cause une affreusen ladie, le delirium tremens, sorte de folie; qi excès de boissons alcooliques procurent aux 1 : cins au moins un quart de leur clientèle. 
Nes bons amis, si vous vous intéressez à nos Proznades, ainsi que j'ai lieu de le croire, nous les ntinuerons pendant la belle saison. Les notions imentaires que vous avez acquises nous permetont d'aller un peu plus vite dans notre étude des nples et de faire une riche collection de "bonnes intes.

FiN DE La Frevière partie. 


$$
\begin{aligned}
& \text { Pewles - } 40=82-148 \\
& \text { Hew } \rightarrow 106-148-36-32
\end{aligned}
$$

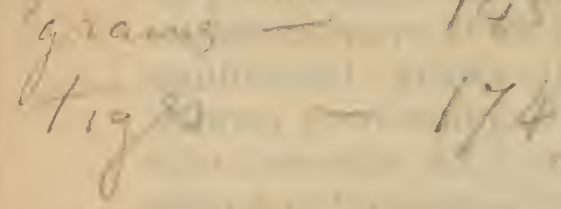

Pwathe theswo of hage $99 ;$ Gem - 14,142 


\section{LES}

\section{REMĖDES DES CHAMPS}

DEUXIÈME PARTIE. 
COULOMMIERS. - TYP. PAUL BRODARD ET Cie. 


\section{DOGTEUR SAFFRAY}

\section{LES}

\section{ZEMÈDES DES CHAMPS}

\section{HERBORISATIONS PRATIQUES}

A L'Usage des instituteurs, Des egClésiastiques ET DE TOUS GEUX QUI DONNENT LEURS SOINS AUX MALADEG

Ouvrage couronné

par la Société libre d'instruction et d'éducation populaires

DEUXIÈME PARTIE

A VRILA SEPTEMBRE

contenant 85 figures

CINQUIÉME ÉDITION

PARIS

LIBRAIRIE HACHETTE ET Cie

79, BOULEVARD SAINT-GERMAIN, 79 



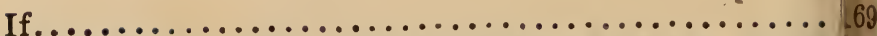

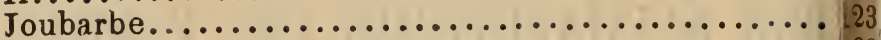

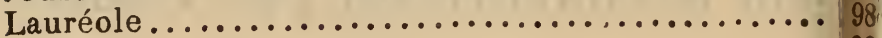

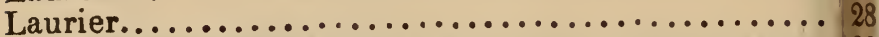

Lierre grimpant......................

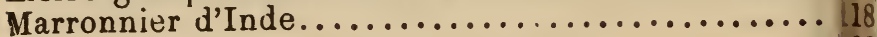

Mézéréon............................. 98

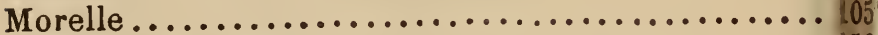

Narcisse des prés.......................... 50

Nerprun ........................... 67

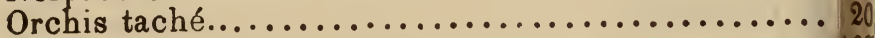

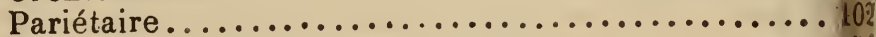

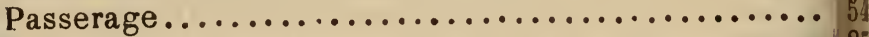

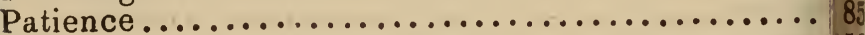

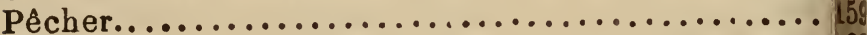

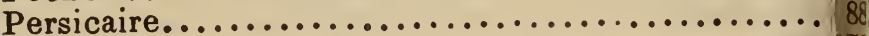

Pervenche.......................... 17]

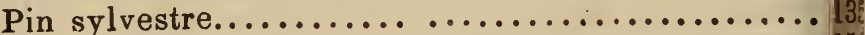

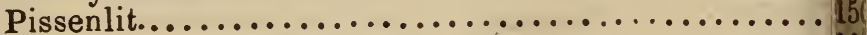

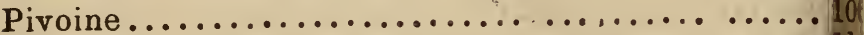

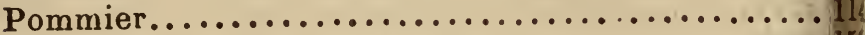

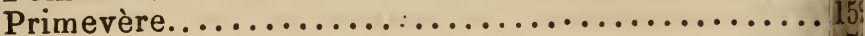

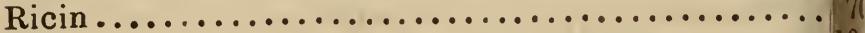

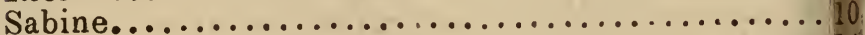

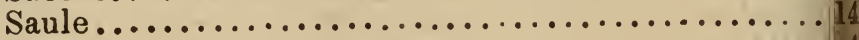

Souci.............................

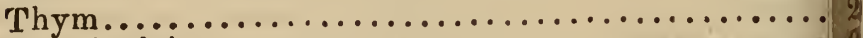

Vermiculaire..........................

Vigne ............................ 17

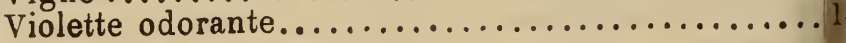


La Clandentuna.

(a)tircon clandestona)

Le la familes des Orobtramohe

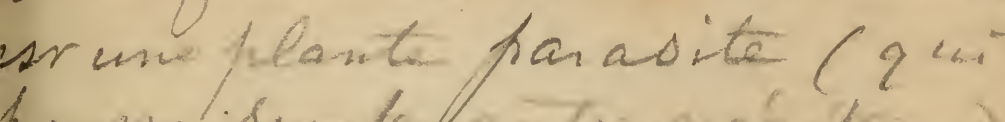

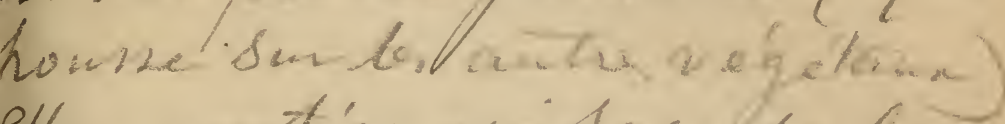
Plle a umetigh quids captwe dazs

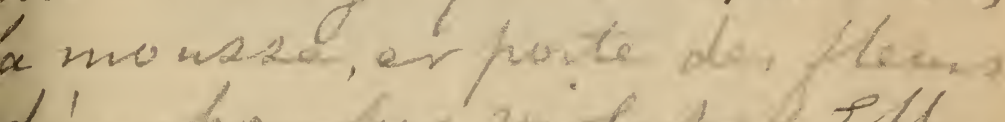
d'm thompus piobet. elle

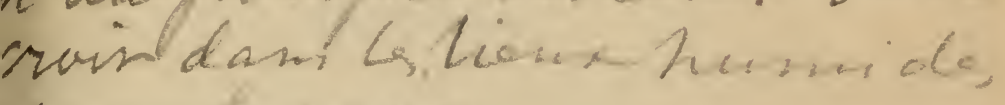
rombat

Coulommiers. - Typ. Paul BRODARD et Cie.

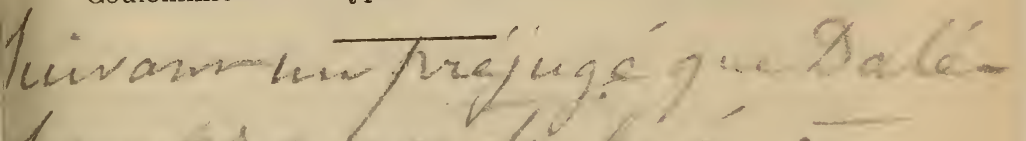

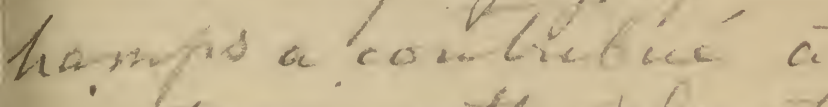
naintemi, cetlo wlamte aw tomifits.

(ow atement.

phopure

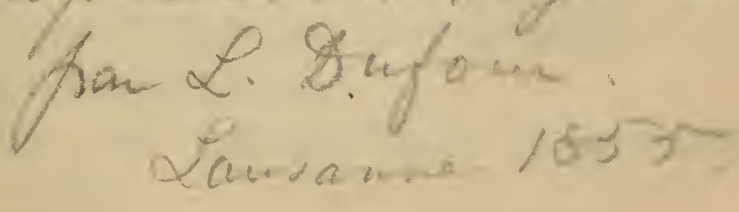





\section{INTRODUGTION}

Le premier volume des « Remèdes des 1amps » est formé de deux séries de Promeides comprenant les mois d'octobre, novembre, icembre, janvier, février et mars. Ce volume ntient deux autres séries, d'avril à octobre, et mplète le cadre de l'ouvrage.

Les éléments les plus indispensables de Botaque, et les notions premières sur l'action des mèdes ayant été, en grande partie, étudiés ns le premier volume, nous pouvons, dans lui-ci, réserver plus de place à l'objet spécial nos herborisations et passer en revue un plus and nombre de plantes.

Toutefois, les richesses de ces mois féconds 
sont si nombreuses que nous serons oblyé d'omettre beaucoup de simples fort intéress at pour ne pas dépasser les limites de ce livre. 


\section{LES}

\section{REMÈDES DES CHA MPS}

TROISIÈME SERRIE DE PROMENADES

(AVRIL, MAI, JUIN.)

Nous entrons, mes amis, dans la saison la plus ropice pour nos excursions dans la campagne. De uelque côté que nous dirigions nos promenades, s prés, les bois, les buissons, les jardins vont ous offrir une abondante moisson de plantes utiles nous constaterons souvent que beaucoup d'entre les joignent, aux qualités solides qui nous les font chercher et étudier, les agréments extérieurs qui us les rendent plus attrayantes. Puisque vous 'ez continué de vous intéresser à nos études penint la saison rigoureuse, alors que la nature n'ofiit à l'observateur superficiel rien qui méritât son tention ou ses suffrages, je suis sûr que les intences bienfaisantes du printemps vont doubler itre zèle et que les impressions reçues dans ces constances favorables laisseront dans votre esit une durable impression. 
Chacuil de vous, je vois, a fait déjà sa cueillett et je n'ai vraiment que l'embarras du choix. Je va mettre à part six ou sept échantillons très-diff rents d'aspect, mais qui présentent tous, bien qu des degrés divers, des prcpriétés semblables.

Vous vous rappelez sans doute les explicatiol que je vous ai données au sujet des remèdes pu gatifs, parmi lesquels nous avons étudié la Belle 1 nuit, l'Ellébore, le Fusain, la Bourdaine, le $\mathrm{Ne}$ prun, le Ricin, la Bryone, etc. Ces médicament vous le savez, augmentent et altèrentd'une maniè! passagère les évacuations intestinales. Mais il y t a qui agissent en même temps sur l'estomac et pr voquent des vomissements, ce sont les émétc-c thartiques. Les plantes que je viens de choisir appa tiennent toutes à cette classe de médicaments.

Commençons par ce beau spécimen que voi avez entendu nommer Flambe, Glaieul bleu, Cou trai, Lirguo, Flamme, mais dont le vrai nom e InIS comiun ou Inis germanique (Iris germanica type de la famille des Iridacées. Cette belle plant à qui il ne manque, pour être recherchée et admiré que d'être exotique, rare et chère, croît spontant ment dans les lieux arides et incultes, les ruine: les toits de chaume, auxquels elle donne un asper riant et pittoresque. Il n'est guère de jardin où l'o n'en trouve des touffes à l'ombre, dans quelque coi perdu. Ce que l'on nomme vulgairement sa racin est une tige souterraine ou rhizôme noueuso épaisse, charnue, blanchâtre, produisant beaucou de chevelu. La tige aérienne, qui atteint de 50 70 centimètres, est garnie à sa base do feuilie planes, aiguës, ensiformes c'est-à-dire en form d'épée. Les grandes et belles fleurs d'un bleu viole 


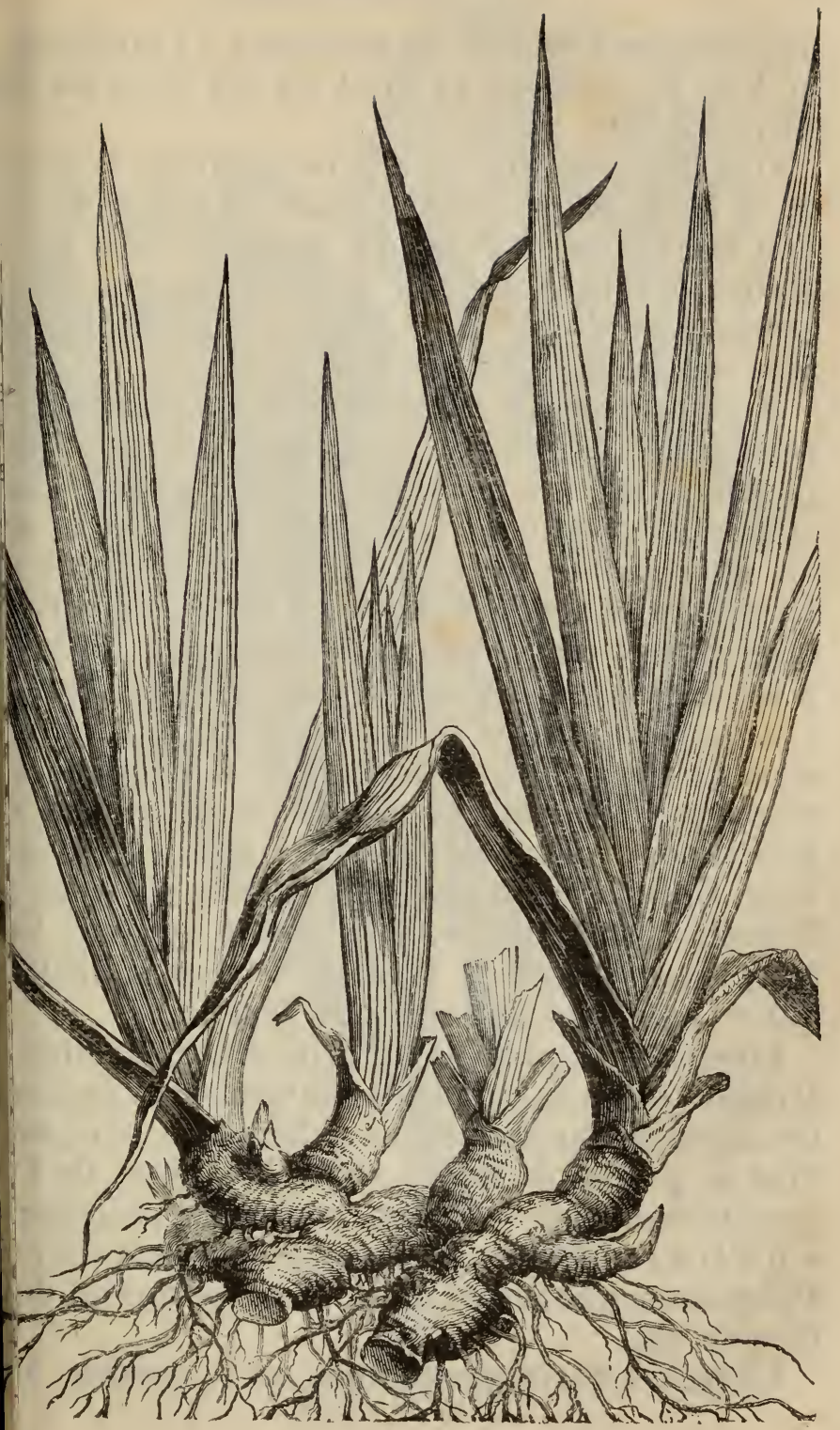

'rge souterraine ou rhizòme de l'Iris, 
agréablement veinées se montrent à l'extrémité la tige, au nombre de cinq ou six dans les $m c$ d'avril et mai.

On peut recueillir en étē le rhizôme, le gratt et le faire sécher promptement au soleil, dans i lieu bien ventilé. Toutefois la dessiccation lui $\mathrm{f}_{c}$ perdre beaucoup de son activité. A l'état frais, exhale une odeur désagréable; sa saveur est amèr âcre, nauséeuse, et un peu styptique.

A petite dose, la racine fraîche est excitante, e pectorante, diurétique et anthelmintique; à do plus forte elle produit des évacuations de l'estom. et des intestins. On l'a employée avec succès das les hydropisies, mais il ne faut pas oublier q1 lorsque cette maladie est liée à des lésions organ ques du foie ou du cœur, les évacuants ne peuvel y apporter qu'un soulagement temporaire. Dans c cas, on peut donner le suc frais de la racine à dose de 15 à 30 grammes, mêlé à une tisane c guimauve, édulcorée et aromatisée. Cette dose do être prise, en deux ou trois fois à une heure d'inte: valle. Si l'on négligeait d'étendre le suc dans un boisson mucilagineuse, il causerait pendant lon temps une sensation de chaleur âcre et brûlant qui en a souvent fait proscrire l'emploi.

Lorsqu'on se propose de produire une action al térante continue, comme il convient dans beaucou. de maladies chroniques. on administre avec avan tage la poudre de racine sèche à la dose de 1 à grammes chaque jour. On peut aussi préparer u vin d'Iris en faisant macérer pendant quelques jour: 120 grammes de racine fraîche broyée-dans un demi litre de vin.

La racine d'Iris germanique sèche possède, à un 


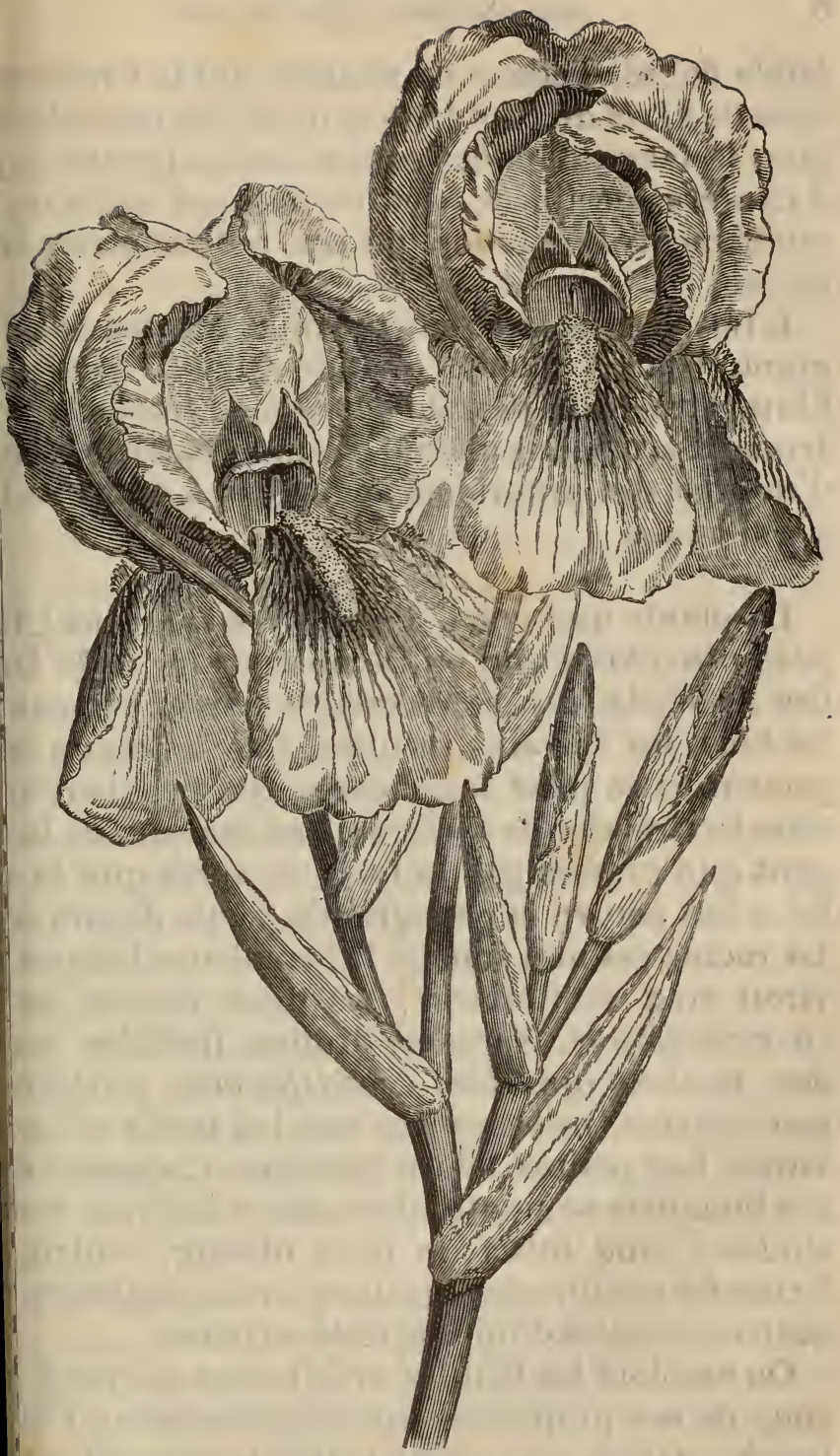

Fleurs a $a^{2}$ ris. 
faible degré, l'odeur de violette qui fait recher(y celle de l'Iris de Florence et ne lui est pas inféric pour la fabrication des petites boules nommées 1 à cautères destinées à entretenir par suite de gonflement, l'irritation de ces petites plaies ar cielles.

L'IRIS FÉTIDE (Iris foetidissima) nommé aussi gigot, Glaieul puant, Spatule, Glaieul sauvage l'IrIs JAUNe (Iris pseudo-acorus) ou Iris des mar Iris faux acore, Iris glaieul, Flamme bâtarde, Flan d'eau ont des propriétés analogues à celles de l', germanique.

La plante que voici, l'Ascié-iade blanche ( $A s$ pias vincetoxicum) ou Dompte-verin, de la fam des Asclépiadées, se rencontre commlnément d les bois, les terrains incultes. Les animaux la pectent, si ce n'est les chèvres qui broutent vol tiers l'extrémité de ses tiges. Les chevaux ne la $\mathrm{n}$ gent que pressés par la faim, et après que la $\mathrm{g}$ lui a fait perdre la plus grande partie de son âcr La racine est une souche tuberculeuse longue $d$ viron cinq centimètres; les tiges, hautes de 4 60 centimètres, simples, droites, flexibles, porl des feuilles opposées, cordiformes, entières, peu coriaces, pubescentes sur les bords et les n vures. Les petites fleurs blanches, 'isposées en tits bouquets semontrent en mai et acint; ce sont étoiles à cinq lobes; le frurs oblong, ventru, $r$ ferme de nombreuses graines ovales, aplatios, $r$ geâtres, munies d'une aigrette soyeuse.

On emploie les feuilles et la racine qui perd he coup de ses propriétés par la dessiccation. Celle fraîche, exhale une odeur nauséabonde qui rappt 
e de la valériane; la saveur, douceâtre d'abord, ient bientôt âcre et amère. On administre la détion de racine à la dose de 15 à 30 grammes par gramme d'eau, pour augmenter la sécréticn des nes ou celle des sueurs; dans les affections daruses, les engorgements lymphatiques et glandu; ; la poudre des feuilles à la dose de $11 / 2$ à rammes comme vomitif.

ette plante ne niérite nullement le nom de npte-Venin que les anciens lui avaient donné à se de prétendues vertus alexipharmaques. On cultive dans les jardins quelques espèces exotis remarquables par les houppes de soie chainte qui accompagnent les graines.

vous ai promis de mêler peu à peu, à l'étude iale que nous faisons des plantes médicinales, 1otions thénriques les plus indispensables pour s initier aux éléments de la Botanique, et, comme $s$ avez pu le remarquer, j'évite de suivre une che didactique et de fatiguer votre mémoire de $s$ qui, sans doute, auraient peine à s'y fixer. Je re vous entretenir aujourd'hui de ce que l'on blle la classification des plantes. C'est d'ordinaire chapitre fort compliqué, aride; mais, comme je point la prétention de vous faire ici un cours 3otanique, je me sens libre de trancher dans le t à ma guise pour l'adapter au but que je me ose.

l'on examine chacun des individus-plantes qui itituent un champ de blé, on reconnaît facilement als sont de même nature, qu'ils appartiennent à la ie espèce, et l'on s'attend à ce qu'ils reproduides individus semblables à eux, Mais la culture, 
le climat et d'autres influences peuvent appor chez quelques individus des différences notabl qui se reproduisent de manière à constituer sous-variètés, des variétés, des races nouvelles.

Si l'on rassemble des espèces dont les traits pr cipaux sont très-rapprochés, par exemple la Ros cent feuilles, l'Eglantine et la Rose du Beng: on formera naturellement le genre Rosier.

Nous avons déjà remarqué des plantes Phané games et Cryptogames, c'est-à-dire dans 1 quelles les organes reproducteurs sont visibles cachés; voilà un élément utile de distinction. étudiant la germination du Haricot, je vous ai remarquer les deux cotylédons ou parties charn qui enveloppent puis nourrissent l'embryon; bien! il y a des plantes, comme la Fougère, Champignon, dont les graines sont dépourvues ces organes; d'autres, comme le Blé, le Lis, l'( chis, l'Arum n'ont qu'un cotylédon et sont ap lées Monocotylédones. Maintenant, parmi plantes Dicotylédones (à deux cotylédons) il y a dont les fleurs n'ont pas de pétales comme l'A toloche, le Plantain; d'autres qui n'en ont qu't par exemple le Bleuet, le Sureau, la Laitue; d' tres enfin qui en ont plusieurs, comme l'Angéliq le Pavot, le Rosier; de sorte que l'on peut étal les grandes divisions de plantes Apétales, Mor pétales, Polypétales, c'est-à-dire sans pétale, $\mathrm{p}$ sédant un, possédant plusieurs pétales. Chaci de ces grandes divisions peut se scinder en class ou familles qui seront reconnaissables à la dis; sition et au nombre des organes de la reprodi tion; telle est, en abrégé, la méthode de classifi tion de Jussieu. 
mme vous voyez, vous saviez déjà une partie que je viens de vous dire; la plupart des mots iques, tirés du grec, vous étaient familiers, de que vous avez pu saisir en quelques instants rincipes, fort simples d'ailleurs, mais qui sont ent une pierre d'achoppement dans les livres iques de Botanique, - Cela dit et retenu, rens à nos plantes.

tte petite Labiée à fleurs purpurines dont les cilles très-rapprochés forment un épi ter1, c'est la BÉtoIne (Betonica officinalis), qui dans les lieux ombragés, les bois, les taillis, rairies. Sa tige, haute de 30 à 60 centimètres, le, droite, carrée, un peu velue, porte des es opposées oblongues, pubescentes, dont les les diminuent en se rapprochant du sommet. est peu d'accord sur ses propriétés. Louée à nce par quelques médecins, elle en a vu res lui refuser toute efficacité. Réduites à leur valeur, ses propriétés n'en sont pas moins assantes. La poudre des feuilles, administrée un jaune d'œuf à la dose de 3 à 6 grammes e heures après la fin de l'accès, dans les fièvres inales rebelles, produit une révulsion sio par un effet éméto-cathartique, après lal'usage des toniques achève la guérison. On uussi employer la poudre de racine à la dose 3 grammes, pour obtenir des évacuations de nac et des intestins. Pour préparer l'infusion uilles la dose est 10 à 20 grammes par kilone d'eau. On emploie la poudre des feuilles e sternutatoire pour combattre les maux de erveux. 
Souhaitons la bienvenue à cette fleur charm des bois, l'une des premières et des plus st productions du printemps. Un parfum qui rap celui de la fleur d'oranger se dégage de ses p fleurs blanches en grelot attachées au nombı douze environ à une hampe grêle, haute de 15 centimètres, embrassée à la base par deux gues et grandes feuilles radicales. C'est lo Mr ou Lis nes Vallées (Convallaria maialis).

On emploie en médecine le rhizôme. les fle et les petites baies tachetées d'abord puis ro qui leur succèdent, on récolte les fleurs au mo où elles s'ouvrent, il faut séparer les pétales $\epsilon$ sécher à l'étuve ou dans un four peu chaud dessiccation détruit leur odeur mais n'en altèr la saveur amə̀re, âcre, nauséeuse, qu'elles comm quent à l'infusion aqueuse. Les baies, et les cines que l'on peut cueillir en toute saison, cè aussi à l'eau des principes amers et âcres : abondants.

On a employé le muguet comme antispasn que, mais avec un succès douteux. Il suffit de tre à profit son action sur l'estomac et sur l'i: tin. La racine surtout a la propriété de faire v et de purger selon les doses que l'on adminis malheureusement on ne règle bien ces doses, chaque cas, qu'après quelques tâtonnements sorte que, s'il n'y a pas de temps à perdre, plus prudent d'employer un éméto-cathart d'un effet plus certain ou plus constant. I grammes de fleurs fraîches broyées avec du produisent d'abondantes évacuations intestini accompagnées de coliques peu durables quo apaise d'ailleurs en prenant quelques tasses 
llon de veau pendant l'action du reméde. Si augmente la dose, on produit des nausées, puis omissement. Ce que nous avons dit au sujet autres remèdes éméto-cathartiques dans le ement des fièvres intermittentes s'applique à ge du Mugust : il agit surtout en causant une rrbation salutaire, et l'on doit compléter ou as- la cure par l'emploi prolongé des toniques s et astringents. Quant aux baies, que l'on a nmandées comme antiépileptiques à la dose à 16 grammes, elles ne doivent guère inspirer onfiance contre cette maladie qui a le triste lége de compter plus de remèdes que toute au. ar cela même qu'on n'en a pas encore trouvé rtain, même pour les genres d'épilepsie qui suceptibles de céder à un traitement rationnel. ésence de cette terrible maladie et de celles qui itent les symptômes, il est impossible de choisir $\mathrm{p}$ sûr, dans chaque cas, le remède qui guérira; pourquoi on est obligé de recourir à des este toute sorte dans l'espoir, fondé d'ailleurs, de er le médicament le mieux approprié à chairconstance.

; fleurs de Muguet, pulvérisées après dessiccaforment comme les feuilles de Bétoinc, un sternutatoire, dont l'action révulsive sur la prane muqueuse du nez est souvent utile dans ouleurs de tête invétérées, les fluxions chronides yeux et des oreilles.

tons en passant que par la culture on a protne variété de Muguet à fleurs plus grandes, on possède deux sous-variétés: l'une blanche rs doubles, l'autre à fleurs simples d'un beau Eiles sont traçantes commo l'espèce sauvage, 
on peut les multiplier comme elle par sépara des touffes après la floraison ou par semis, exc pour la sous-variété double.

Mes amis, lorsqu'on n'a vu les plantes mé nales que dans la boutique de l'herboriste, on magine facilement que les plus laides sont les n leures, et que les vertus plus ou moins occie qu'on leur suppose, n'agissant d'ailleurs que une série de sensations désagréables, excluent $t$ idée de beauté, de grâce, de fraîcheur, dans végétaux-remèdes. Il faut avouer que cela est quelquefois, mais aujourd'hui, vous voyez nous pouvons former un vrai bouquet champ avec les sujets que j'ai triés pour notre causi Que chacun de vous prenne en main une de branches fleuries de Genêt; leurs belles graje jaunes méritent de nous occuper quelque ter Vous l'avez peut-être entendu nommer Sparti balai, Genettier, Juniesse; pour nous, c'es GenEtT a BalaI (Spartium scoparium) de la fan des Légumineuses.

Avant d'aller plus loin, je veux vous dire $q$ ques mots de cette famille très-intéressante, quelle appartiennent le Haricot, le Sainfoin, l'Ace la Lentille, le Trèfle, la Luzerne. Toutes ces gumineuses sont réunies sous la dénominatiol Papilionacées à cause de la forme caractéristi de leurs fleurs que nous allons étudier en détai

Si vous examinez attentivement la fleur de l' de ces plantes, vous verrez qu'elles se rappor toutes à un type qui vous est bien familier, la f du Pois cultivé. Comme vous voyez, la prem pièce de la corolle est un grand pétale évasé, fe: 
haut, qui semble destiné à recouvrir, à protéger autres parties : on le nomme le pavillon ou endard. Si vous détachez ce pétale, vous remarz qu'il est emboîté solidement avec les deux
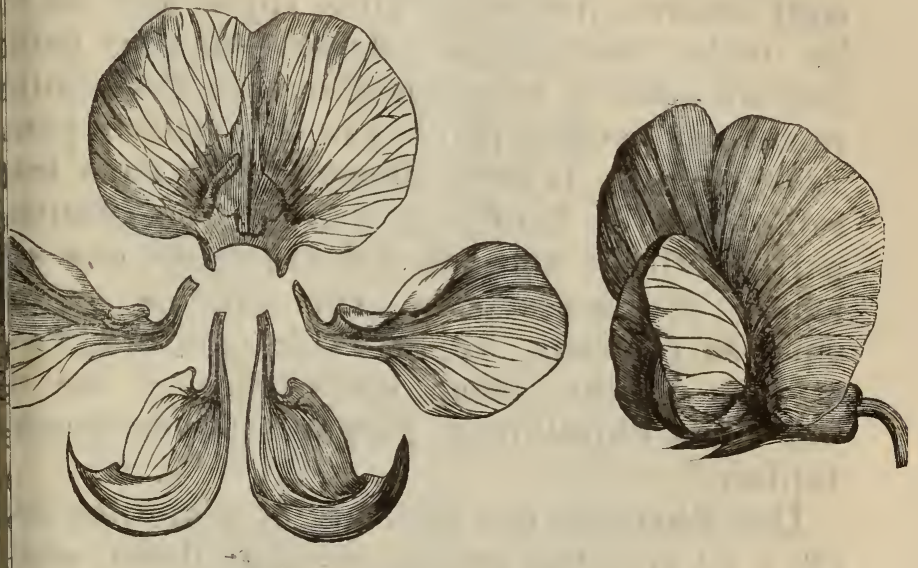

Corolle papilionacéo

les inférieurs ou ailes qui se réunissent pự mer les organes de reproduction soutenus à artie inférieure par deux pièces recourbées ne la proue d'une barque, d'où leur nom de 2e. Si nous continuons à disséquer la fleur, trouvons, dans le genêt, dix étamines, un 'e surmonté d'un style simple un peu courbé rminé par un stigmate. Le fruit est, comme du pois, une gousse dans laquelle les graines ittachées par un pédoncule nommé funicule. rme particulière de fruit appelée gousse est le caractéristique des légumineuses, et la forme our que nous venons d'étudior a fait ranger 
toutes les légumineuses qui en sont pourvues cin la tribu des Papilionacées, dont le Genêt estu représentant digne d'intérêt à tous égards.

Le Genêt est un sous-arbrisseau, c'est-à-dire u sa tige est dure, ligneuse, tandis quo ses ramtu sont tendres, herbacés. Vous voyez que ses feil les ovales, pubescentes, deviennent plus petit mesure qu'elies se rapprochent de l'extrémitéd rameau et perdent peu à peu leur pétiole pousle venir sessiles. Il croît abondamment dans les rains secs et fleurit en mai et juin. Toutesie parties offrent une odeur dósagréable, une sa amère et nauséabonde. On emploie en $m$ cine les rameaux, les fleurs, les graines, l'écc, et l'on obtient, selon les parties et les d, des effets diurétiques, purgatifs ou éméto-cat tiques.

Une décoction des rameaux et sommités fler (30 à 60 grammes par kilogramme d'eau) admi trée à dose altérante ou légèrement laxative e: trutes les sécrétions, notamment celle des uri et peut rendre de grands services dans le rhi tisme chronique, la goutte, les scrofules, les n dies chroniques du foie et de la peau; à dose forte, c'est un des meilleurs remèdes à essayer les infiltrations aqueuses et les diverses fol d'hydropisie. Pour cette dernière maladie, o: trouve très bien de l'usage d'un vin préparé 500 grammes de cendres de genêi dans 2 grammes de vin blanc, à la dose do 125 gram chaque matin. Mais ce remède, comme tous "I qui augmentent l'activité des reins, serait nuis si ces organes étaient le siòge d'inflammation. Io tion thérapeutique des cendres de genêt est d 


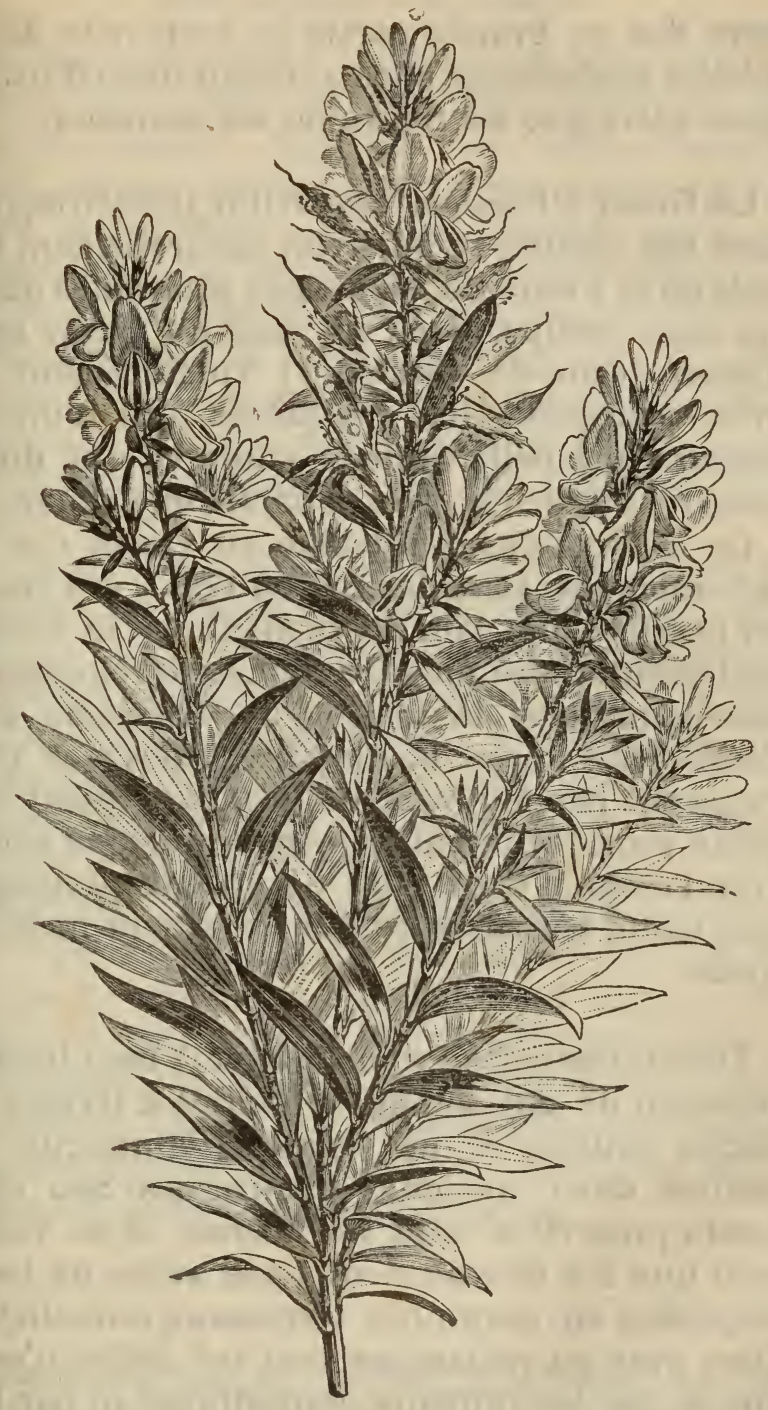

Sommité de Genêt. 
leurs due en grande partie au carbonate de sidi qu'elles renferment, elles agissent done d'une f:ol toute autre que les fleurs ou les rameaux.

Le Genêt d'Espagne (Spartium junceum), cuiv dans nos jardins et qui croît naturellenent dail midi de la France, a les mêmes propriétés que di qui nous occupe. Il en est ainsi du GeNÊT DES II TURIERs (Genista tinctoria) vulgairement Gris trole, Spargelle, Herbe à jaunir, qui fournit teinture une belle couleur jaune vif ; et $d u G$ pUrGatif ou Genêt griot (Spartium purgans).

On a faussement attribué au Genêt des ve anti-rabiques. Dans les cas où l'on a cru réuss est possible que l'on doive attribuer le succès cautérisation des pustules qui se développent cl nairement sous la langue du troisième au vième jour après la morsure. Mais ces pust n'existent pas toujours, et jusqu'à présent il r qu'un remède qui puisse mériter quelque confia c'est l'emploi d'un bain de vapeur prolongé, plus haute température que le patient pourra porter.

Terminons cette promenade déjà bien longue l'examen de ces somnités fleuries d'Hyìble $(S$, bucus ebulus) et de Sureau (Sambucus nig) nommé dans quelques campagnes Séu et Sí Leurs propriétés étant les mêmes, je ne vous $]^{\text {th }}$ lerai que du Sureau dont nous avons ici les fles disposées en corymbes terminaux ombelliforns. Vous avez pu remarquer que cet arbre n'est qué ni par les animaux domestiques ni par les nilles. On dit que ses baies qui sont purgati, 
ent les poules et que ses fleurs sont funestes aux ndons. Vous savez du reste que ses feuilles, lors'on les froisse, dégagent une odeur très-désaéable, et que l'odeur des fleurs fraîches est naueuse, mais devient presque agréablo après leur issiccation. L'infusion de ces fleurs sèches, à la se de 4 à 15 grammes par kilogramme d'eau, est i sudorifique d'un emploi vulgaire, tandis qu'une coction de fleurs fraîches agit comme diurétique purgatif ainsi que celle des feuilles ( 30 grammes r kilogramme d'eau) qui est d'ailleurs moins itée.

La partie du Sureau qui a le plus d'énergie à tat frais est la seconde écorce, c'est-à-dire l'écorce rte qui se trouve immédiatement au-dessous de piderme grisâtre. Une décoction de 60 à 70 gram. is dans un kilogramme d'eau, coupée avec moitié t et administrée quatre ou cinq fois par jour à ses croissantes de 60 à 100 grammes, a souvent nué de bons résultats dans l'hydropisie; il en est même du vin de sureau préparé en faisant inser 150 grammes de seconde écorce dans un kiloamme de vin blanc. On peut aussi donner le suc l'écorce par doses de 15 à 60 grammes par jour. bur obtenir un résultat, il faut causer de copieuses acuations. Toutes ces préparations, bien que spéhlement purgatives agissent aussi comme vomitif sque les doses sont un peu fortes.

Laissez-nıoi choisir d'abord dans votre cueillette tte petite plante remarquable par son odeur de uc qui croit sur les vieux murs, les décombres, 
les lieux frais et incultes, où elle fleurit pend: toute la belle saison. Vous l'entendrez apper Herbe à Robert, Bec de grue, Géraine robert

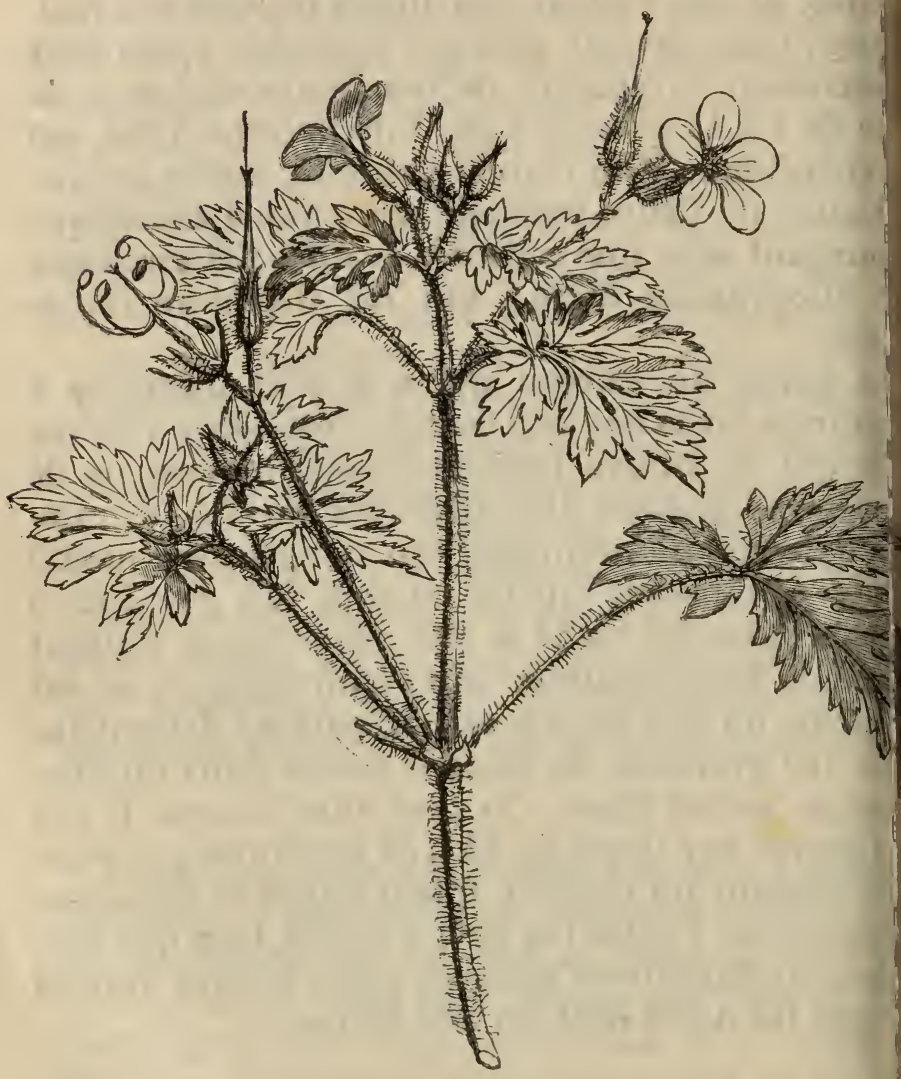

Géranion.

Herbe à l'esquinancie. Pour nous, c'est le Geranil (Geranium Robertianum), plante bisannuelle) 30 à 35 centimètres, aux rameaux rougeâtres, a: 
ars rosées, dont le fruit velu se termine en bec ongé, type de la famille des Géraniacées.

y'est un astringent faible, usité parfois en garisme dans l'angine et à l'intérieur dans les hérrhagies; la dose est de 15 à 30 grammes dans grammes d'eau.

;i vous examinez attentivement le fruit du Géra$n$, vous verrez que les graines sont renfermées is de petites loges enchâssées au bas d'un axe ngé et soutenues par des filets qui partent du amet de l'axe. A la maturité, les loges s'entrount et les filets se recourbant subitement vers le amet, lancent les graines de manière à les semer ne certaine distance.

Tous pouvez admirer dans beaucoup de plantes ilières cette prévision de la nature pour la dishination des graines par la force mécanique déoppée à un moment donné par les parties élastiis du fruit, et vous avez sans doute provoqué jouant cette détente des fruits de la Balsahe.

le que vous me présentez ici est un membre de amille des Fougères, nommé parfois Adiante, Jveux de Vénus, qui croît abondamment dans lieux humides et ombreux, surtout dans le midi l la France, et dont les feuilles ont acquis en méine une réputation fort exagérée. La Capillaire liantum capillus Veneris) est légèrement stypiue et amère, son odeur agréable se développe is son infusion. C'est un remède vulgairement ployé comme béchique dans les affections de la trine, mais assez insignifiant. On appelle béques des médicaments doux, émollients, cal- 
mants qui apaisent la toux et les irritations dea poitrine.

Voici un autre remède béchique, mais plus $u_{\theta}$ parce que ses propriétés sont plus actives et p nombreuses, c'est le Tussilage (Tussilago farfar de la famille des Composées, appelé aussi Pas d'â Pas de cheval, Herbe de Saint-Quirin, ou de Sai Guérin, Taconnet, Procheton; c'est une plante vace qui se trouve dans les terrains argileux, as bords des fossés, des ruisseaux, les tiges sct des hampes simples, longues de 10 à 15 centin tres, garnies de nombreuses écailles et qui porte chacune en avril-mai une fleur d'un heau jau de soufre à laquella succède un fruit (akèr oblong, cylindrique, couronné par une aigret Les feuilles, radicales, pétiolées, cordiformi lisses, dentées, d'un beau vert en dessus, sont bla châtres et cotonneuses en dessous, et ne se montre qu'après les fleurs; pour les conserver il faut 1 dessécher avec soin, car l'humidité les détrı promptement.

Les fleurs d'une odeur forte, agréable, d'une s veur douce et aromatique, sont rangées parmi l espèces pectorales ou béchiques, comme la Mauv le Bouillon blanc, la Violette. Quant aux feuille que l'on emploie en décoction à la dose do 60 100 grammes et plus par litre d'eau, elles passaie depuis longtemps pour efficaces dans les affection scrofuleuses, lorsqu'il s'éleva sur leurs vertus di doutes exagérés qui ne sont pas encore bannis c l'esprit des médecins. Des expériences conscier cieuses reprises depuis quelques années nnt prour qu'elles constituent une ressource précieuse, pot 
anipdgnes, dans la scrofule et ses complications rses, soit seule, soit idée par l'usage du vin entiane. Le traitement est toujours long, quels soient les remèdes employés, aussi ne faut-il se rebuter après les premiers essais. On fera de donner chaque jour 60 grammes de suc feuilles, en augmentant progressivement jus100 grammes. Pou* boisson on préparera une ction de 50 grammes de feuilles sèches dans un ,ramme d'eau, à prendre dans la journée. Pour ge externe, le Tussilage est avantageusement jlacé par des plantes plus énergiques, comme exemple les feuilles et les sommités de la saire dont nous allons maintenant nous occuper.

SaponarRe (Saponaria officinalis), de la famille Caryophyllees, vulgairement Saponière, Saère, Herbe à foulon, est une plante vivace, mune auprès des rivières, des ruisseaux, des is, des haies, etc. D'une racine grèle, d'un blanc âtre, allongée, rampante, s'élève une tige hero peu rameuse, haute d'environ 60 centimègarnie de feuilles opposées, lisses, d'un vert re, et terminée par un corymbe-ombelliforme lies fleurs blanches ou rosées à cinq pétales, jaraissent en juillet-août. Toute la plante conune substance nommée saponine, soluble dans , à laquelle elle communique toutes les proés de l'eau de savon; aussi l'emploie-t-on pour chir le linge et enlever les taches des vêtets. On s'en servait autrefois pour préparer les es de laine avart la teinture, d'où son nom rbe à foulon. La saponine se rencontre aussi le Mouron rouge, l'Eillet, l'Arum, les jeunes 
pousses de Pomme de terre et dans un grand nom de végétaux exotiques.

La culture a produit deux variétés de Sapona à fleurs simples et semi-doubles, elles demancn un sol frais et un peu d'ombre.

Cette plante est spécialement dépurative, c'esà dire capable .non pas de délivrer l'organismed quelque matière impure qui s'y trouve toute forre comme on le croyait autrefois, mais capabled donner aux tissus, par son action tonique, ap tive, fondante et légèrement diurétique, la forcd sécréter et d'absorber dans des conditions normes C'est en effet un agent excitant, tonique, prop augmenter les sécrétions et à stimuler les fonct de nos organes. On comprend dès lors qu'elle utile dans les maladies chroniques, comme adjur du traitement spécial, ainsi que dans les convas cences. Elle rend parfois de grands services ca les affections dartreuses, et son emploie n'cr aucun danger.

Pour préparer la decoction on emploie de 30 grammes de racine broyée, ou de feuilles or racines sèches, par kilogramme d'eau.

Ce que nous venons de dire au sujet des priétés médicales de la Saponaire s'applique, grande partie, à celles de la petite plante que ris avons ici, la Fumeterre (Fumaria officinalis) do la famille des Fumariacées, qui est tonique, in dante, dépurative et légèrement vermifuge. l'emploie avec avantage dans le traitement des ladies des viscères, des scrofules et des maladieile la peau qui ne réclament pas l'emploi de remi spéciaux, comme les dartres. Elle convient $t$ 
aux enfants atteints de faiblesse des organes istifs, de croûtes de lait, d'affections vermises. Cependant la Fumeterre n'agit dans ce dercas que par ses propriétés toniques et par son rtume; elle ne serait pas assez énergique s'il issait de débarrasser rapidement l'intestin de

qui causeraient des accidents inquiétants. ime la Fumeterre croît abondamment dans les nps, les terres cultivées, les vignes, on peut la tituer au Trèfle d'eau comme antiscorbutique, ù il est difficile de se procurer cette plante.

es vaches et les moutons la broutent malgré amertume, mais elle est délaissée par les cois et les chevaux. Vous l'avez peut-être enlue nommer Fiel de terre ou Pied de geline. est facile à reconnaître : la racine blanche, tante, fibreuse, donne naissance à une tige ce, étalée, longue de 25 à 30 centimètres, portant rès-petites feuilles d'un vert bleuâtre ou cendré, mposées, et dont la disposition rappelle celle feuilles de Persil. La fleur, d'un bleu rougeâtre, etée de pourpre au sommet, forme des grappes inales lâches qui se montrent de mai à oce.

n récolte la Fumeterre un peu avant l'éclosion fleurs, et on la dessèche promptement. Le suc imé se donne à la dose de 60 à 100 grammes, ou mêlé au petit-lait, on prépare un sirop en outant son poids de sucre. Pour l'infusion ou écoction on emploie de 30 à 60 grammes de la te sèche par litre d'eau, de bière ou de vin.

y a quelques autres espèces de Fumeterre, e autres la Fumeterre bui.Beuse (Fumaria bull) dont la racine qui contient de l'amidon est 
mangée par les Kalmoucks, mais elles ne remla cent pas bien celle dont je viens de vous parles

Voici encore une plante qui jouit de propr té dépuratives, mais à un degré moindre que les ré cédentes : c'est la Pensée sauvage (Viola tricor) ou Petite jacée, Fleur de la Trinité, Herbe clavelée, qui vous est trop familière pour $9 j \mathrm{j}$ besoin de description. Elle appartient à la farll des Violariées. On récolte l'herbe entière et fíri pendant toute la belle saison, et il faut preir soin de la dessécher promptement.

On prépare l'infusion avec 30 à 60 grammed plante sèche par litre d'eau. Elle semble spécle ment ứile dans les croûtes de lait, cette maili commune de l'enfance qu'il faut s'empressesd guérir aussitôt qu'elle se manifeste, malgré le sé jugé populaire qui veut qu'on les respecte coln un travail utile de la nature. Pour cela, onai macérer pendant la nuit de 4 à 8 grammes de pl t sèche dans 250 grammes d'eau chaude; le mi on fait bouillir, on coupe avec un quart de sucré et l'on administre à jeun. Il faut donne remède pendant deux uu trois semaines.

La racine de Pensée sauvage est émétique, corn celle de Violette.

Voici, mes amis, une plante plus connue dan ei jardins potagers qu'à l'état sauvage, mais qui, pendant, croît spontanément dans les terrains lé $r$ et sablonneux : c'est l'Aspenge (Asparagus offic a lis), de la famille des Liliacées.

La souche, que les jardiniers appellent griffe,s formée d'un faisceau de racines jaunâtres ou 
r, grosses comme une plume d'oie. Au prina)s elle donne naissance à des tiges souterraines

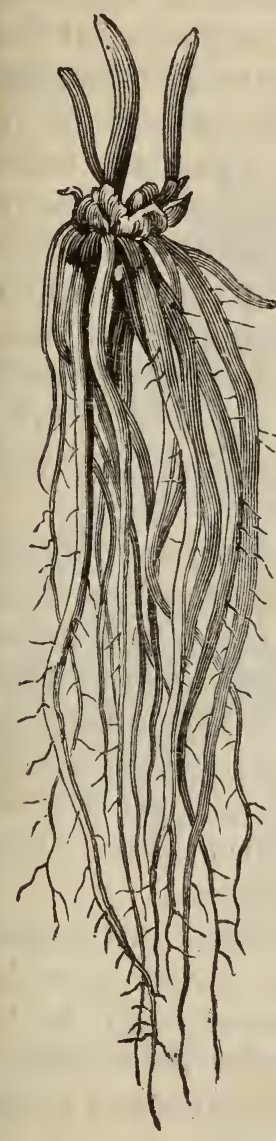

$$
\text { Asperge. }
$$

Griffe.

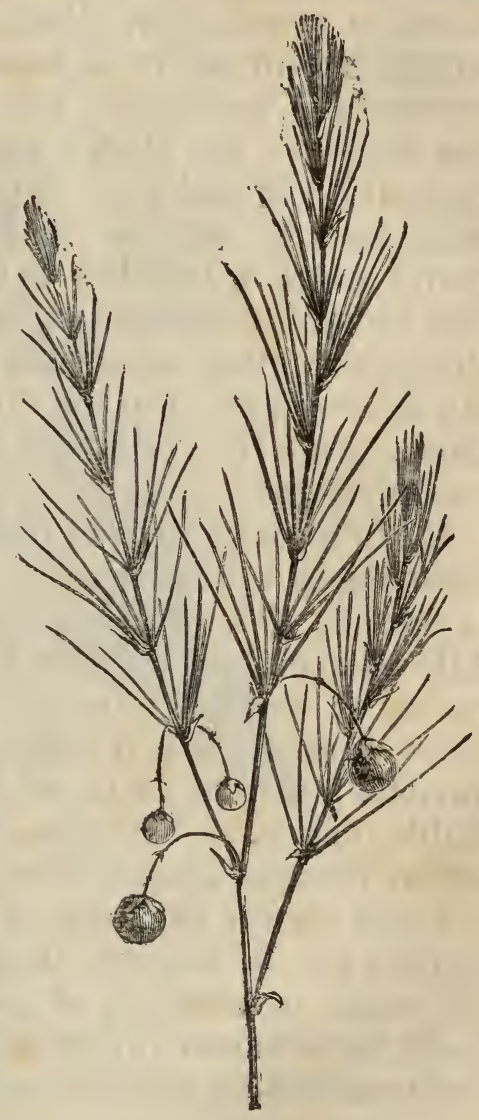

Rameau

driques, écailleuses, nommées turions, qui so inent par un bouton légèrement conique formé 
par la réunion d'écailles qui recouvrent les idi ments des rameaux. Au lieu de couper les ti l'état de turions pour l'usage de la table, si o le laisse se développer, elles atteignent au-dessu di sol, plus d'un mètre de hauteur. Les bouquetsili formes qui garnissent les rameaux sont de jus ses feuilles, ou plutôt des rameaux secondre partant de l'aisselle de la feuille véritable qui on siste en une simple petite écaille brunâtre. fleurs mâles et femelles se trouvent d'ordinaireiu des individus différents, ce sont donc des flur dioïques; elles consistent en petites clochett six découpures, qui produisent une baie glou leuse, d'abord verte, qui devient d'un rougevi en mûrissant.

Il n'y a guère qu'une préparation d'asperge t'́ ressante en médecine, c'est la décoction de racis la dose de 15 à 60 grammes par kilngramme d'vo Elle possède la propriété d'activer la sécrétion ri naire, et comme tous les diurétiques, serait sible dans le cas d'inflammation des reins. turions jouissent de la même propriété, mais à1 faible degré. L'asperge donne aux urines n odeur caractéristique désagréable.

Cette plante est une de celles qui s'amélion le plus par la culture, et qui récompense le mix les soins industrieux et persévérants. Le jardie peut transformer un sauvageon sans valeur enı des produits les plus recherchés de nos tables.

Je vous ai dit tout à l'heuro que les fleurst l'Asperge étaient dioïques, c'est-à-dire que les flec de sexe différent étaient portées par des pla1 différentes. Voici maintenant une plante à fle 
roïques c'est-à-dire de sexe mâle et femelle sur eul pied, comme nous l'avons vu dans la ciille; c'est l'ORTIe (Urtica), vulgairement Ortie ohe, Petite Ortie, Ortie brûlante, type de la llo des Urticées; mais dans cette mêmo famille,

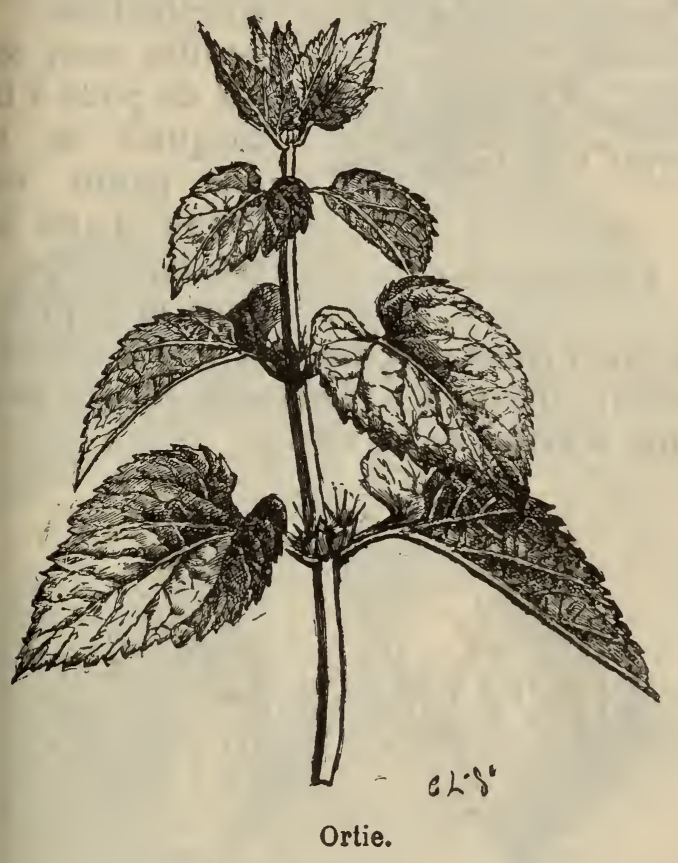

trouvons l'Ortie dioïque.(Urtica dioica), vulment Grande Ortie, Ortie vivace, dans laquelle leurs de sexe différent sont séparées comme Is de l'asperge.

is deux espèces croissent abondamment dans lécombres, les lieux incultes, les haies. La PeJrtie possède à un bien plus haut degrè que la 
grande, la propriété de produire sur la pealu cuisson doulourelise accompagnée de déman son, dont la médecine se sert parfois comme $r$

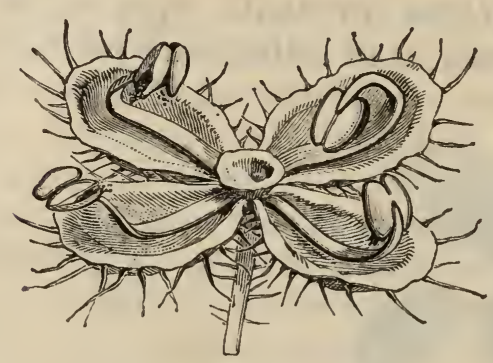

Fleur mâle d'Ortie. sif et dérivatif. a les douleurs $\mathrm{rhm}$ tismales, sous le d'urtication. feuilles sont core tes de poils à lava desquels se tuu une petite vésu pleine d'une lice caustique qui il dans la piqûre, or que les poils pénétrant dans la peau, comprie le réservoir. Toutefois l'habitudo rend à peu in insensible à l'effet de ce venin.
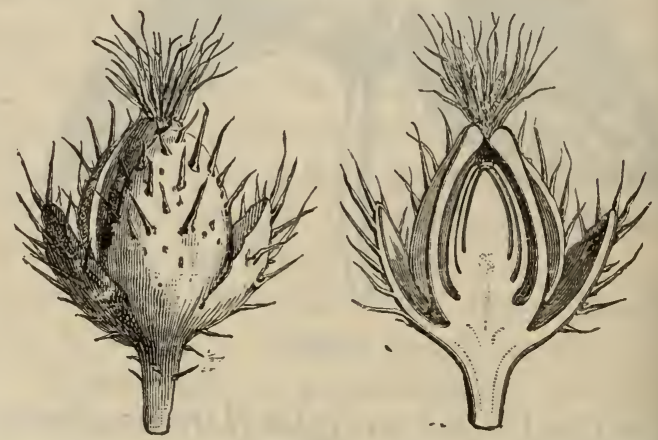

Fleur femelle d'Ortie, sntiere et coupée.

La seule propriété médicale indiscutable petite et de la grande Ortie, c'est d'arrêter praf tement les hémorrhagies. Pour cela, on cin surtout le suc des tiges et des feuilles à la do 


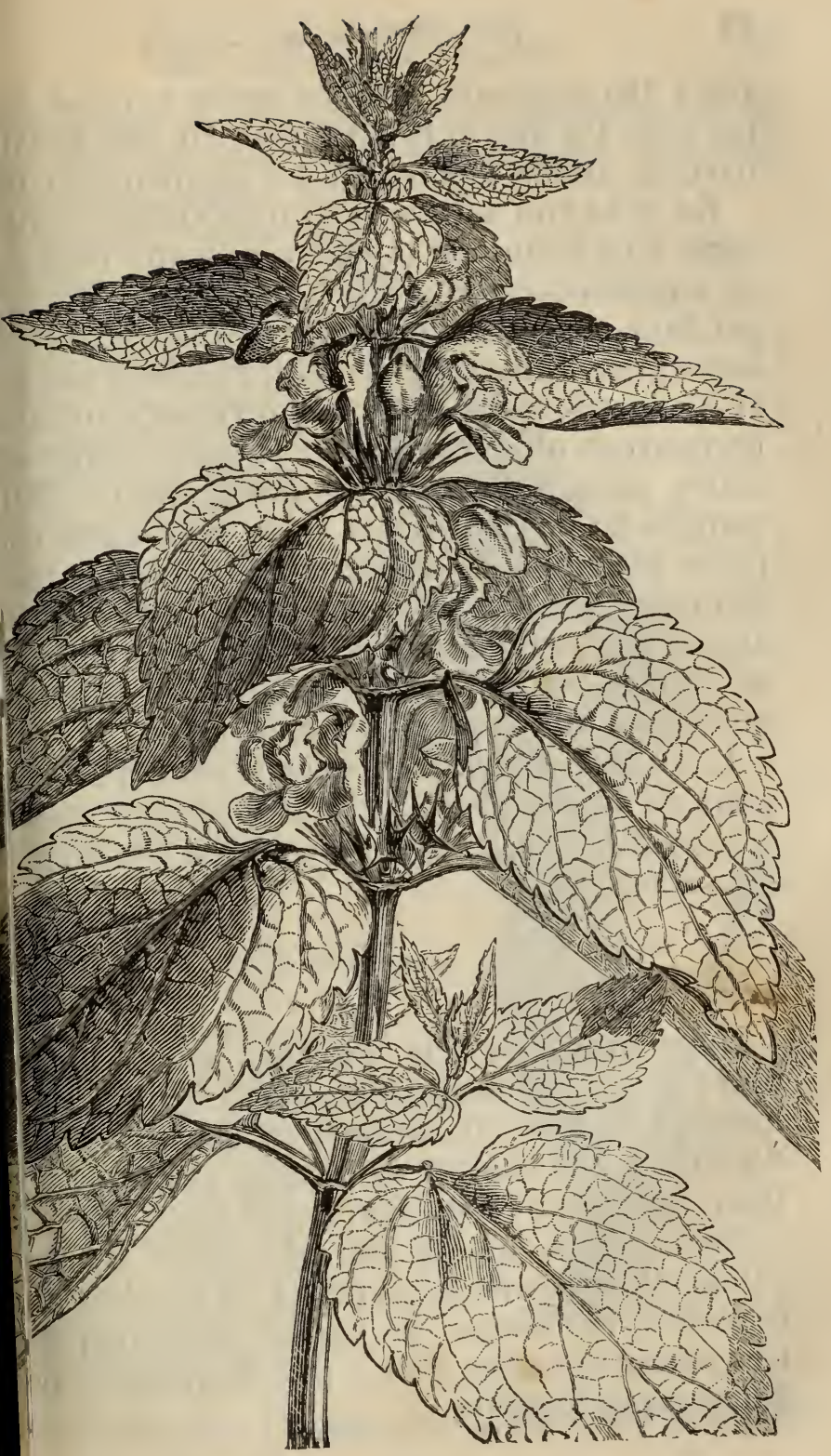

Lamiar blane ou Ortie blanche. 
100 à 200 grammes, répétée assez souvent, sura les cas. Ce suc introduit sur du coton dar lo narines, arrête facilement les saignements dene En Suède on cultive la Grande Ortie commeou rage. Elle donne de la nourrituro aux bestiau di le commencement du printemps, et l'on er fa pendant l'été deux ou trois coupes que l'on serve pour l'hiver. Les volailles aiment ses gr.n et mangent ses feuilles après qu'elles sont fan's ne peuvent plus leur piquer le palais, celles-c sor utiles surtout aux jeunes dindonneaux. Enf peuples du Nord retirent de la racine une co et jaune et emploient les fibres des tiges à la pla chanvre. Vous voyez, mes amis, que malgrisc abord peu attrayant, cette plante si vulgail $\theta$ une de celles qu'il nous importe de connaît que l'on n'utilise pas assez chez nous.

Il ne faut pas confondre les plantes que notv nons de décrire avec l'Ortie blanche qui appa à la famille des Labiées, appelée en botaniqul MIER BLANG.

Nous avons ici une des plus belles plant: notre climat, remarquable par ses feuilles sag longues de 25 à 30 centimètres, souvent marı de taches blanchâtres et brunes, et par la $\mathrm{g}$ fleur qui termine la tige. Cette fleur est cact ristique de la famille des Aroidées. Au lic calice elle présente une spathe ou enveloppe braneuse en forme de cornet, dans laquel trouve un spadice formé par la réunion di ganes reproducteurs. 
'Arum (Arum maculatum) a reçu les noms de iet, Pied de veau, Vaguette, Langue de bouf, erbe à pain, Raciimidonnière, HerIragone. C'est une ate vivace com. ne dans les lieux nides. Sa racine un rhizômecharbrunâtreà l'extéIr, blanc intérieuent, imprégné suc laiteux très, de saveur brûe, mais dont les priétés nuisibles araissent par l'éition ou la torréion, de sorte que peut alors le ger sans crainte in retire un amide qualité supére. Les feuilles hes, contuses, ; employées pour luire la rubéion ou la vésica- A l'intérieur on inistre surtout cine d'un an, qui a plus énergique. doit autant que

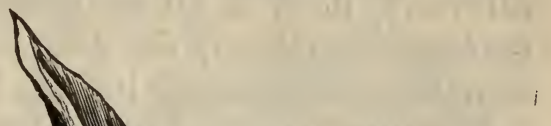
sible l'employerfraîche, car la dessiccation lui fait 
perdre beaucoup de son activité. Elle rend des r. vices incontestables à la dose de 1 à 2 grammes come altérant; de 4 à 10 grammes comme purgatiou éméto-cathartique, dans de l'eau de gomme oulu mucilage de graine de lin. On l'emploie avec suc s, à dose un peu forte, dansle rhumatisme, mais suriut dans les affections chroniques des organes respatoires. Du reste, il ne faut pas oublier que l'Arm est un poison violent, et l'on doit veiller à ce quees enfants n'aient pas occasion d'y goûter. Reteer d'ailleurs que l'Oseille possède la propriété de r. traliser le principe âcre de l'Arum, de l'Euphoee de la Bryone, du Garou, et qu'il suffit d'en mâcer pour faire disparaitre l'inflammation de la boule produite par leur contact.

Je vous ai parlé plusieurs fois de remèdes a rants, et nous allons avoir aujourd'hui l'occasn d'en étudier plusieurs, aussi je veux vous donnı leur sujet quelques mots d'explication. On apple ainsi des médicaments qui exercent sur l'éconoie une action insensible d'abord, lente, continue, le manière à altérer les conditions de vitalité organes. Ils sont spécialement utiles dans les fort nombreux où les désordres causés dans l'ornisation, ne se manifestent pas avec les symptồ d'une maladie bien déterminée, et dans beaucip de maladies chroniques.

Ne négligeons pas cette humble plante qui cit partout, dans les lieux cultivés, les décombres, terrains pierreux. Les noms un peu gauloís lui th śté prodigués, on l'appelle Foirolle, Chiole, Car relle, Caquenlit, Rinberge. Pour nous, c'est a Mercuriale (Mercurialis annua), de la famille 


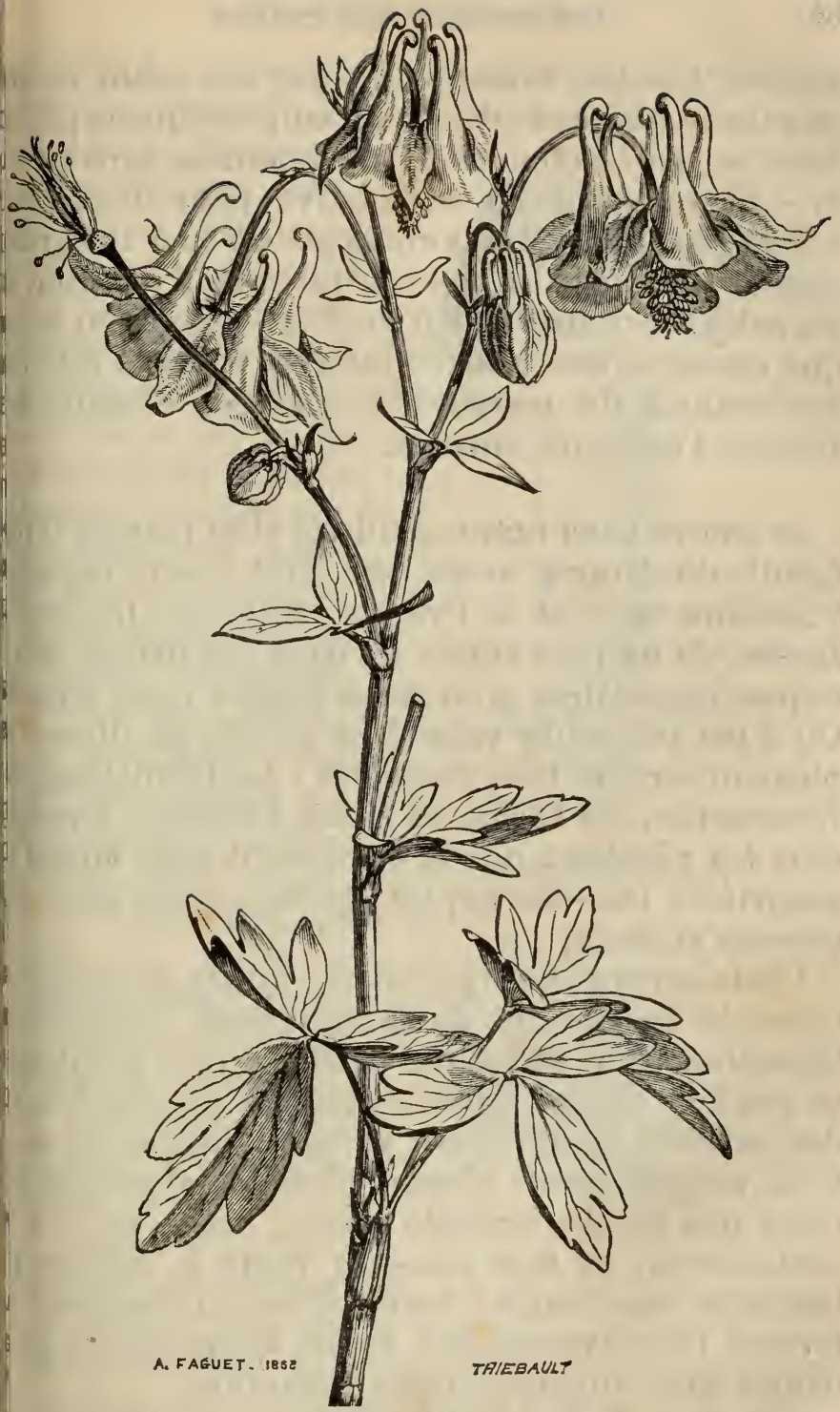

Sommité d'Ancolie. 
Euphorbiacées, romarquable par son odeur fétic, sa saveur amère et salée. On n'emploie que la plar' fraîche. La décoction de 20 à 50 grammes dans un c. mi-kilogramme d'eau esi laxátive; pour obtenir u action purgative, il faut employer de 30 à 100 gra. mes de suc. Dans les caś très-rares où il est utile rappeler les croûtes de lait dont la suppression bri. que causerait une maladie interne, il parait que c3 applications de mercuriale contuse, chaude, $\mathrm{ft}^{\mathrm{t}}$ revenir l'affection cutanée.

Je trouve dans notre cueilletto cinq plantes d'us famille dont nous avons déjà étudié deux espèce: l'Ellébore noir, et la Pivoine : celle des Renonc. lacées. Je ne puis entrer ici dans les détails bol. niques nécessaires pour vous décrire cette famil, Qu'il me suffise de vous dire qu'elle se divise plusieurs tribus bien distinctes : les Clématites, Renoncules, les Ellébores et les Pivoines. Presq. tous les végétaux qui la comrosent sont doués propriétés très-énergiques, quelques-uns sont is poisons violents.

Choisissons d'abord l'Ancolre (Aquilegia vulgar) nommée aussi Gant de Notre-Dame, Aiglantir, Colombine, qui se trouve dans les bois montue et que l'on cultive dans les jardins où elle fourlb des variétés nombreuses, à fleurs simples ou $d c$. bles, rouges, roses, bleues, blanches et panaché, C'est une plante herbacée vivace, haute de 50 à centimètres; sa tige rameuse porte à différen's hauteurs des feuilles blanchâtres en dessous, formes très-diverses. Ses belles fleurs bleues pe. dantes sont employées dans la teinture.

Les racines, les feuilles, les fleurs, les grain 
ossèdent des propriétés diaphorétiques, très-prores à exciter l'évolution normale des maladies ruptives lorsqu'ello $\theta$ semble pas suffiamment développée ur la peau. On emloie la poudre de selences à la dose de 2 4 grammes, en émulon dans du jaune 'œuf, ou l'on fait une fusion de 4 à 8 grames dans un demitre d'eau. L'infusion un ou deux grammes fleurs, édulcorée, it comme sédatif ns l'irritation des onches, et calme no. blement la toux. Elle ninue aussi, à son but, l'effervescence rile. On multiplie incolie par semences par éclats de pieds, automne : elle aime ie exposition omI. hée et la terre de lyère.

le vous dirai peu de c) se au sujet do ce

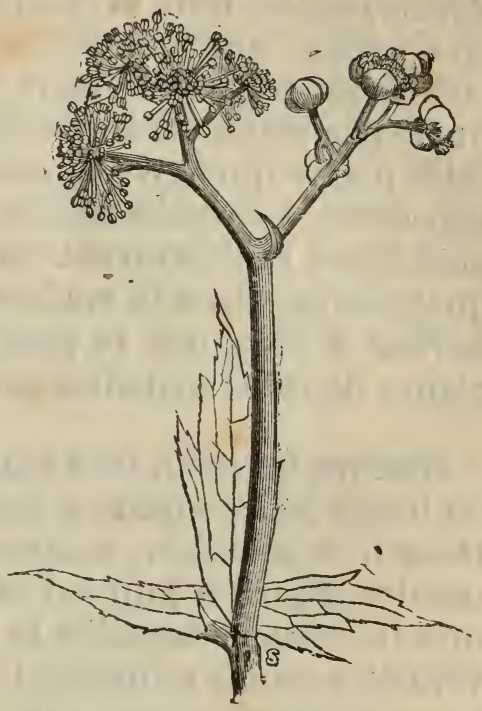

Sommité d'Actẻe.

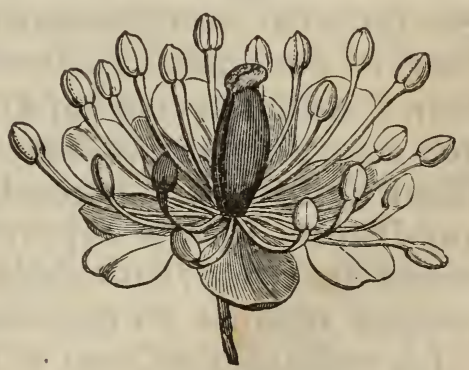

Fleur d'Actée. Scimen d'Aćtée (Actæa spicata), Herbe de Saini(ristophe, Faux Ellébore noir, Herbe aux poux, 
plante vivace des bois ombragés et montueux qu atteint de 40 à 80 centimètres, à feuilles découpées décomposées, d'un vert foncé au dessus, blanchâtr. en dessous, qui montre, en mai-juin, des grappe compactes de petites fleurs blanches. Il est pruder de ne pas employer à l'intérieur les préparations d cette plante qui agit à la manière des poisons narec tico-âcres. La racine fraîche est un purgatif violex analogue à l'Ellébore noir, dont les vétérinaires use quelquefois. Dans la médecine humaine, on doit borner à employer la poudre et la décoction de plante dans les maladies parasitaires.

Remarquez bien, mes amis, cette plante couver de longs poils soyeux à feuilles radicales deux a trois fois pennées, comme celles du Persil, q montre d'avril à juin à l'extrémité de chaque tiş, une fleur dans laquelle le calice d'un bleu viol velu et soyeux, remplace la corolle. Elle habite lieux arides, les bois sablonneux, les coteaux c: caires, où l'on doit la récolter avant la floraisc. Ses propriétés, extrêmement actives lorsqu'elle $t$ fraîche, diminuent de beaucoup par la dessio tion. C'est la Pulsatille (Anemone pulsatilla), cc nue sous les noms de Coquelourde, Herbe au ve Fleur de Pâques, Teigne-CEuf, Passe-fleur, Fle aux-Dames. Elle se rapproche beaucoup de l' $A 1$ mone des prés (Pulsatilla nigricans) et les des plantes rendent en médecine les mêmes serviç.

La pulsatille est âcre et irritante. Pilée fraîchest appliquée sur la peau, elle produit de la rouge: puis la vésication, et enfin l'ulcération de la par Les paysans l'emploient ainsi autour des poignts, comme sinapisme, pour guérir les fièvres interr 
ntes, mais elle ne peut agir efficacement que si on a au préalable administré un vomitif et un purtif. Le suc frais produit sur la langue un sentient d'âcreté brûlante, il suffit de le respirer pour ovoquer l'éternuement, et la poudre des fleurs et es feuilles sèches est un bon sternutatoire. Il sem. e que la Pulsatille agisse d'une manière très-effico dans la coqueluche. On emploie l'infusion de grammes, au plus, de feuilles fraîches, dans 200 rammes d'eau édulcorée. La même préparation, ̀̀ oses répétées, est utile contre les maladies dareuses, mais il faut en surveiller l'effet.

Pour ce qui concerne la Clématrte des hates 'lematis vitalba), qui vous est famillière sous l'un is noms de Cranquillier, Aubervigne, Berceau de Vierge, Viorne, Vigne blanche, Herbe aux gueux, n'ai qu'un conseil à vous donner, c'est de vous stenir de l'employer en médecine, si ce n'est mme vésicant, à la manière de la Pulsatille, car n'est pas encore bien fixé sur ses propriétés qui nt très-actives, et par conséquent dangereuses tre des mains inexpérimentées. Son nom d'Herbe $x$ gueux vient de l'usage qu'en font certains menints pour former des ulcères superficiels afin httirer la commisération sur une fausse infirmité.

Peu de plantes ont reçu autant de noms que le que je tiens ici. On l'appelle suivant les proices ou les cantons, Coqueluchon, Capuchon, ore, Madriélet, Capuce de moine, Tue-Loup, Pistets, Napel. En botanique, c'est l'Aconit NapeL (conitum napellus), plante vivace qui croît dans lieux ombragés, principalement sur les monta- 
gnes. On la cultive dans les jardins, mais elle evrait en être bannie, car de toutes les Renoncracées c'est la plus énergique et la plus dangere e. Toutefois, comme elle a été étudiée avec soin, lo peut rendre en médecine de grands services.

La racine noirâtre ressemble assez à un Itit

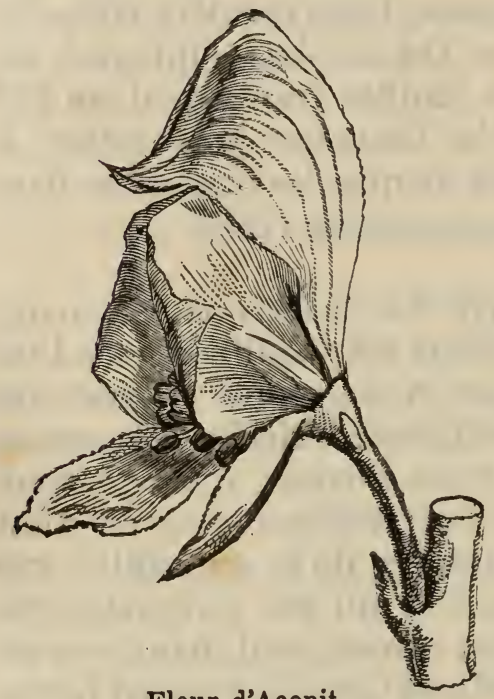

Fleur d'Aconit.

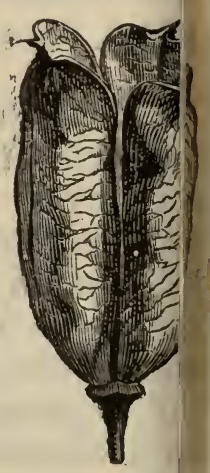

Fruit d'Acor

navet (d'oủ son nom latin) accompagné de rhizĉles latéraux, les feuilles sont profondément inciséter lanières étroites. Les fleurs disposées en grajel terminales allongées, sont remarquables par la f(mt d'une portion du calice nommée casçue qui rou: vre comme un capuchon les deux ailes ou péles latéraux. Le gynécée, ou ensemble des organt io melles, se développe en un fruit formé de trois $2 r$. pèlles, sortes de cornets membraneux qui rejor. ment les graines. 


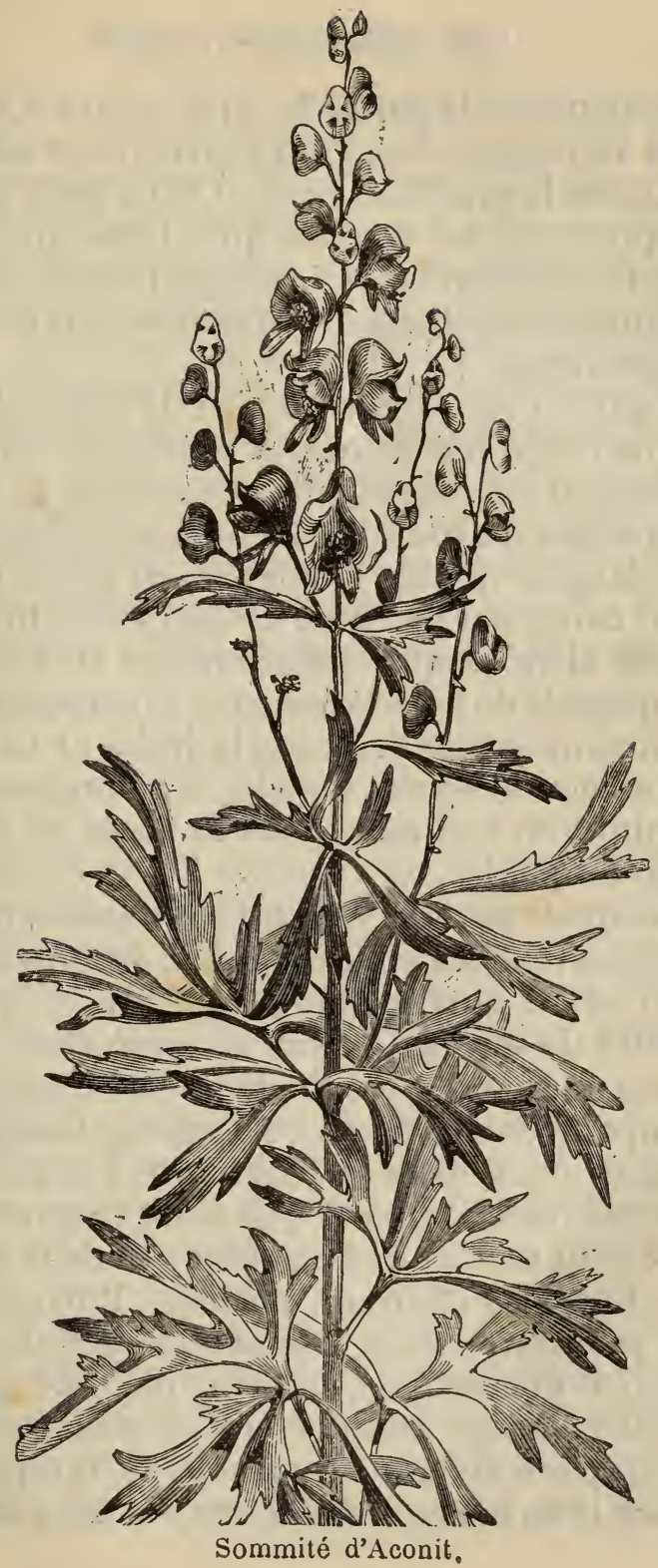


La racine est la partie la plus active. On la colte à l'automne. Celle qui provient des montagis du midi est la plus énergique. On ne peut emplo $r$ avec précision les feuilles qu'à l'état frais, car $\theta$ mode de dessiccation et le temps pendant lequel n les a conservées changent complétement leur valir thérapeutique.

On devra commencer l'usage de cette plante $\mathrm{r}$ des quantités très-faibles et surveiller son effe à mesure que l'on augmentera les doses. Il n'y a is à s'inquiéter du fourmillement que produit l'Accit sur la langue et dans la bouche, ni même de l'alttement causé par une dose un peu forte, mais on e doit pas aller jusqu'à produire de la somnole:e accompagnée de frissonnements. L'empoisonnemit par l'Áconit se manifeste par la froideur de la pei, des douleurs violentes de tête, des vomissementst la diminution très-notable de la force et de la :2́. quence du pouls.

On emploiesouventl'Aconit avec succès contre :s névralgies rébelles à l'Opium, à la Jusquiame et Belladone; il agit comme stupéfiant de la doul par suite de son action sur la circulation et sule système nerveux; c'est de la même manière qil se comporte dans quelques maladies inflammatoils, principalement dans le rhumatisme aigu. Come les autres Renonculacées que nous venons d'exa ner, il peut rendre de bons services dans la coce luche. Entre les mains du médecin, l'Aconit est $1 \theta$ arme puissante, mais elle est dangereuse danla médecine domestique, et l'on ne peut guère n conseiller l'usage aux personnes inexpérimentés

On emploie la teinture alcoolique (préparée a'c une partie de racine fraîche broyée pour cinq dl- 
ol) à la dose de 1/2 à 2 grammes, l'extrait aqueux a dose de 5 à 30 centigrammes, ce sont les seules éparations qu'il soit utile de vous indiquer. Comme nous ne manquons pas de remèdes énergies sans être dangereux, nous devons nous applier à les bien connaître, afin d'en tirer tout le parti ssible, et ne pas nous laisser entraîner, par l'atit des difficultés vaincues et ces dangers évités, user sans une préparation scientifique suffisante, s moyens d'action qui réclament des connaissanapprofondies. Les notions pratiques, élémentaique je vous donne dans nos entretiens, excluent détails techniques sans lesquels l'emploi de cerns végétaux actifs pourrait causer de terribles sidents.

Nous allons nous occuper spécialement aujourui d'une famille fort intéressante, dont beauip d'individus vous sont familiers, et dont les actères botaniques sont simples et bien définis, veux parler des Crucifères dont nous avons ici nombreux échantillons.

e prendrai pour type la Moutarde. Le nom Crucifères donné à cette famille vient de la dispition en croix des quatre pétales, dont se como la corolle. Ce sont presque toutes des plantes bacées; beaucoup d'entre elles sont alimentaicomme le Chou potager, le Chou-fleur, le Navet, Rave; le Radis, le Cresson; d'autres, comme la oflée, le Thlaspi, la Julienne, sont cultivées pour rs fleurs; le Colza fournit une huile employéo Is l'éclairage; on retire de la Guède un principe 
colorant bleu, nommé pastel. Un des caractèreses plus saillants des Crucifères, c'est la dispositior le leur fruit qui consiste en une membrane épase sur laquelle sont attachées les graines, recouverte ar
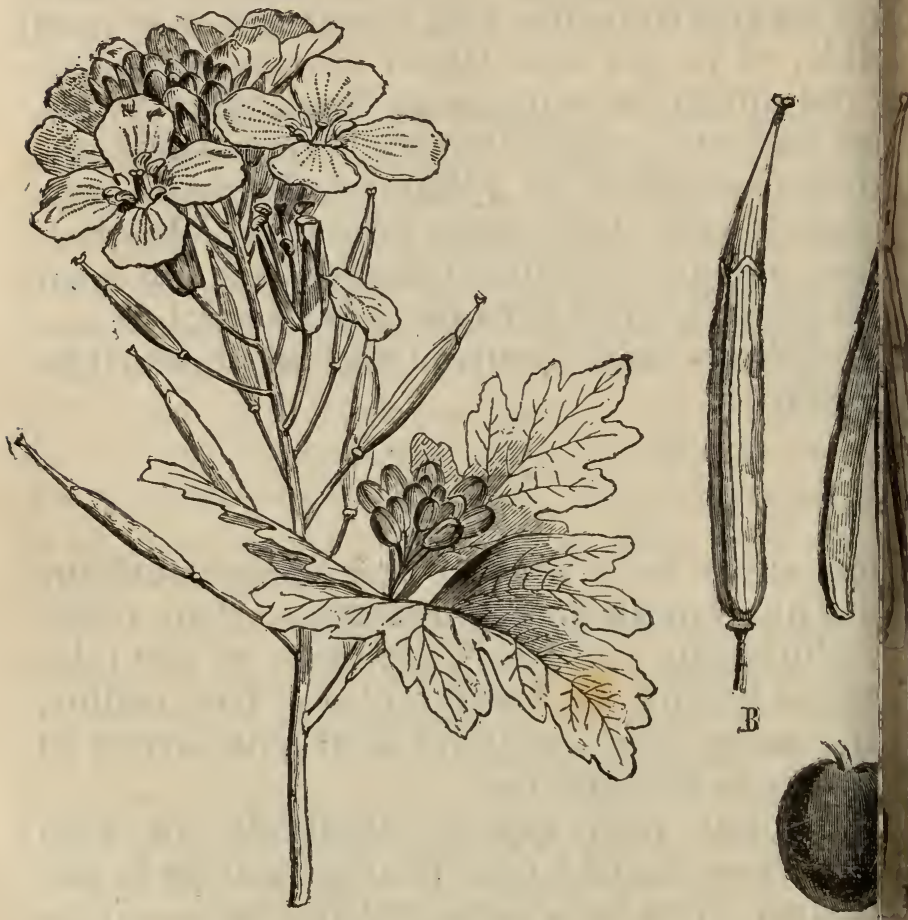

Moutarde sauvage.

E, silique; D, grse.

deux valves qui s'écurtent à l'époquo de leur m rité : on appelle siliques les fruits de cette na ro qui se retrouvent du reste dans d'autres famille Co spécimen que vous avez récolté appartie la Moutarde sauvage (Sinapis arvenis), plante veie, 
leurs jaunes, très-abondante dans nos champs, il est presque impossible de la détruire, mais l'on n'emploie pas en médecine, parce que ses priétés sont moins énergiques que celles de la UTARDE NOIRE (Sinapis nigra) qui est l'objet d'une ture assez importante, On la sème à la volée au ntemps, dans une terre douce, légère et bien eublie et on la récolte vers la fin d'août.

Luand on écrase la graine de moutarde on y renait une odeur assez forte et une saveur âcre siquante, mais pour développer l'huile volatile constitue son principe spécial, il faut l'imor d'eau à la température de 10 à 30 degrés. it à la présence de cette huile volatile que la ine de Moutarde doit sa propriété la plus impore, que l'on met en œuvre lorsque l'on emploie sinapismes ou cataplasmes de cette graine lue, pour produire la rubéfaction de la peau me médication révulsive, c'est-à-dire capable létourner des parties profondes le cours du sang r l'appeler à la surface, et aussi de produire un sement dans certains organes par l'action nere mise en jeu en certains points. Il faut bien arder de préparer les sinapismes avec de l'eau grée, à moins que l'on ne veuille diminuer tion de la Moutarde. L'application de ce remède lier réclame une certaine surveillance, car son n. trop prolongée pourrait donner lieu à des lents surtout chez les enfants ou chez les peres dont la sensibilité est émoussée par la malaOn emploie les sinapismes pour produire une ation générale dans les fièvres de mauvaise re, alors qu'au mouvement fébrile a succédé orostration extrême et inquiétante; dans la pad 
ralysie, l'apoplexie; pour produire vers la surty la dérivation de quelques affections, commeli goutte, le rhumatisme, les dartres; enfin pour cin battre la douleur dans des cas de point de côté névralgies, ou pour rappeler des écoulements sp primés. Au lieu de l'employer en cataplasmes, la mêle parfois à l'eau de maniluves ou de pédilu's La graine de moutarde pulvérisée et mêlée àlı vin blanc est très-utile dans le scorbut, mali dont je vais vous parler tout à l'heure. On l'a c $n$ née avec avantage ( 15 grammes en décoction ćn 150 grammes d'eau) dans la fièvre putride malig̨e et aussi dans la période de calme des fièvres in mittentes, l'angine, le rhumatisme chronicie L'huile douce que l'on retire des semences est jr gative à la dose de 60 grammes et anthelmintile Lorsqu'on a besoin d'un vomitif prompt et in comme dans les cas d'empoisonnement, on aci nistre une cuillerée de farine de graine de $\mathrm{mu}$ tarde dans un verre d'eau. On en fait aussi un gargarisme contre l'angine tonsillaire.

Mais si la moutarde rend de grands servicesu personnes lymphatiques et aux malades épuisés la souffrance, elle serait nuisible aux sujets veux, irritables, disposés à l'inflammation et congestions.

On administre quelquefois, une cuillerée d graines de moutarde, entières, comme laxatif les cas d'atonie de l'intestin chez les hypocon driaques, les paralytiques, les vieillards, mai ol lui préfère, pour cet usage, les graines de bo TARDE BLANGHE (Sinapis alba) qui sont moitiélu grosses. Le charlatanisme qui s'annonce danle journaux a fait un sujet de réclame des prote 
$s$ inconstestables mais limitées de cette graine et transformée en une panacée; vous saurez mainte. nt l'apprécier à sa juste valeur.!Pour préparer le ndiment nommé moutarde, on fait macérer penint trois ou quatre jours les graines de Moutarde ire dans une quantité de vinaigre suffisante pour mouiller, puis on les broie au moulin ou dans mortier : on peut y ajouter de l'Estragon, du rsil, du Cerfeuil, de l'Ail, de la Cannelle pour tenir un produit aromatisé.

Je vous disais il y a quelques instants, que le vin Moutarde était utile contre le Scorbut; je tiens vous donner ici quelques indications sur cette ladie, dans laquelle sont utilisées toutes les plandont nous allons nous occuper aujourd'hui. Le rbut consiste principalement dans l'appauvrispent du sang, causé par un mauvais régime con. ué pendant un temps assez long. Le manque ir, d'exercice et de soleil, joint à une nourriture เ variée suffit pour développer cette maladie qui it surtout au printemps. L'usage prolongé des ades salées et la privation de légumes frais renont autrefois le scorbut redoutable pendant les ages au long cours; aujourd'hui, les marins en ffrent beaucoup moins parce que l'emploi des umes conservés, du vinaigre, des citrons, des imes de terre crues, retarde ou prévient le déppement de la maladie. Les symptômes prinux sont le gonflement des pieds, la bouffissure a face qui prend une teinte jaune, l'engorgement gencives qui deviennent saignantes et ulcérées, aignements de nez, enfin des taches rouge-viois de sang retenu dans les vaisseaux superficiels. 
Vous connaissez tous lo Cressom (Sisymbriu nasturtium), je n'ai pas besoin de vous le décrir I) contient du fer, de l'iode, du soufre, des pho phates, c'est donc une plante riche en minéraux;

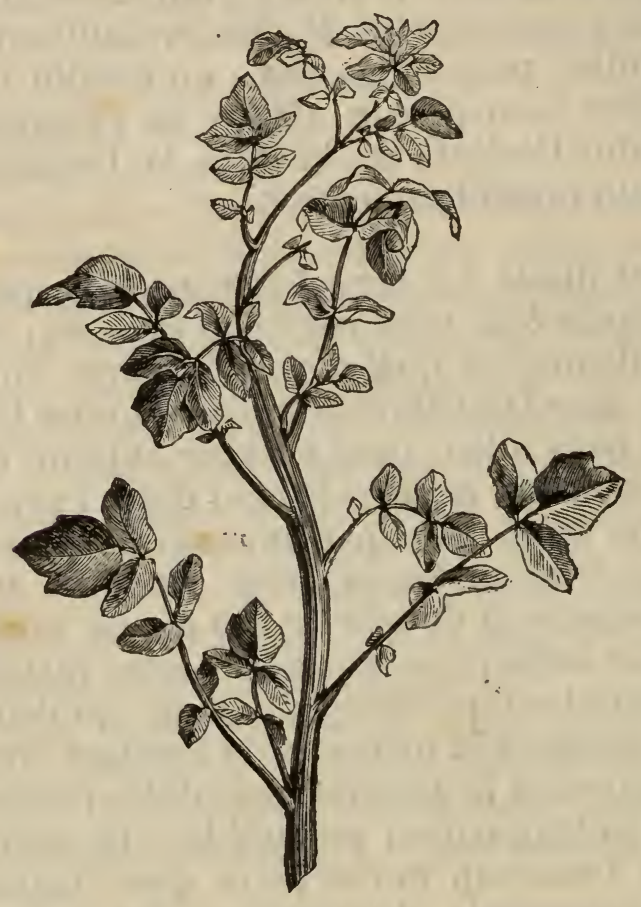

\section{Cresson,}

est stimulant, diurétique, diaphorétique et an corbutique, utile dans les engorgements de la 1 qui accompagnent les fièvres intermittentes ou li succèdent; dans les scrofules, certaines hydropis et dans une foule de maladies chroniques. Si l'or xaange la plante en nature; le suc exprimé es 
meilleure préparation; on le donne à la dosede 60 à 150 grammes coupé avec du lait, du petit it ou d'autres sucs d'herbes. On le mâche pour iffermir les gencives ramollies et ulcérées par le sır. but. Il est avantageux d'einployer, en même te:ps que le Cresson, les hoissons ou les plantes acidus: cidre, Oseille, Épine-vinette.

Ce que je viens de vous dire se rapporte ćg.ementau Cochléaria (Cochlearia officinalis) ou Hibe aux cuillers, Cranson, Herbe au scorbut, qui flerit en mai-juillet dans les lieux humides, au borcide la mer et sur les montagnes; à la Cardanine ( $(x$. damine pratensis), dont vous avez remarqué ins doute, au printemps, les jolies fleurs d'un bne rosé ou lilas, disposées en corymbe ou en grapt terminale, nommée aussi Cresson des prés, Creior élégant, Cresson sauvage, Passerage sauvage et au Raifort sauvage (Cochlearia armoracia)ou Moutarde des capucins, Moutarde des Allemals Radis de cheval, Cran de Bretagne, Rave saur;e qui croît spontanément dans les fossés, au borcles ruisseaux, et que l'on cultive dans les jardins emploie surtout sa racine forte, charnue, très- $n$. gue, d'un blanc jaunâtre à l'extérieur, blanch er dedans, qui contient une huile assez semblable ju les propriétés à celles de la Moutarde. Après l'apil râpée, on l'emploie comme assaisonnement oulor en prépare du vin, de la bière, un sirop à la de 30 à 40 grammes par kilogramme de liqule C'est aussi un bon rubéfiant.

Le Vélar (Erisymum officinale) ou Sinapi, ot telle, Moutarde des haies, Herbe-au-chantre, est ne plante annuelle qui croît spontanément dansles 
lieux incultes, au bord des chemins, où elle monts en mai-juin ses très-petites fleurs jaunâtres dispsées en épi grêle, est une auil

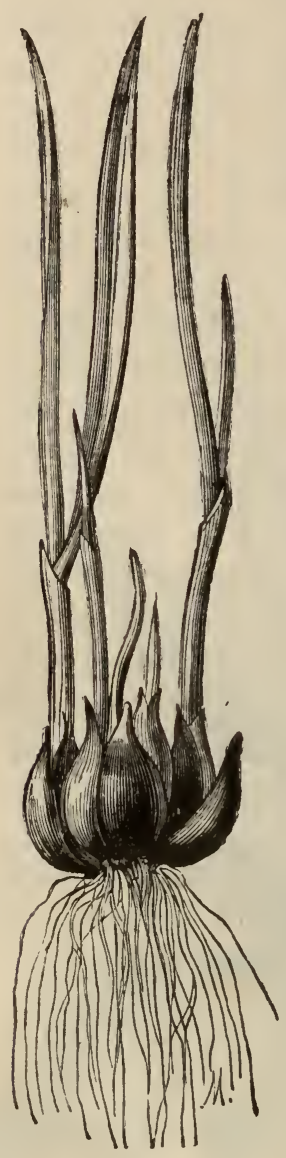

Ail. crucifère qui, inusitée dans a médecine urbaine, n'en mér pas moins son ancienne répul. tion populaire dans l'enrov: ment, les extinctions de voix $t$ les affections catarrhales. C'it une des rares plantes de ce 3 famille qui ne perd qu'une ass faible partie de ses propriés par la dessiccation. Toutefois $!$ vaut mieux l'employer fraîcl. On donne le suc à la dose dé à 30 grammes; on prépare l'i. fusion avec 30 à 60 grammes $\mathrm{pr}$ litre d'eau.

Je vous mentionnerai en pesant une autre crucifère, l'A. LIAIRE (Erisymum alliaria) Herbe aux aulx, Julienne, Vé.r alliaire, qui jouit des propriéts déjà étudiées cans les individs de cette famille, et qui contict la même essence que l'AiL ( $t$ lium sativum), de la famille (s Liliacées, dont je vais vous (. tretenir pour terminer cette pymenade.

Le bulbe de l'Ail contient $u_{3}$ huile volatile d'une saveur piquante et chauc, d'une odeur caractéristique extrêmement diffusib 
ui a de l'analogie, quant à ses effets, avec celle de Moutarde.

On a tour à tour vanté et abandonné l'usage de Ail dans presque toutes les maladies. Autrefois n lui attribuait la vertu d'éloigner les maléfices, omme on le croit encore capable de préserver des pidémies. Pris à dose ordinaire, comme assaisonement, il augmente l'appétit et favorise la digeson ; son action excitante se manifeste par une plus rande activité des organes sécréteurs et absorants ; peut-être que c'est simplement par ses proriétés toniques qu'il préserve des fièvres paludéenes ou qu'il les guérit; c'est d'ailleurs un bon ermifuge. On peut employer contre la fièvre d'acis ou contre les vers, une décoction de 4 à 15 rammes par 500 grammes d'eau ou de lait. On l'a onné avec succès dans le croup et dans la période gide (de refroidissement) du choléra. Dans ce cas, l'applique pilé en frictions et en cataplasmes. 'est un rubéfiant et vésicant fort utile, et l'on use omme antiseptique, pour le pansement des plaies 3 mauvaise nature, des ulcères, un vinaigre d'ail réparé par infusion. Enfin, appliqué sur les cors xx pieds, un bulbe d'ail écrasé détruit le reste de talité de la partie dégénérée que l'on détache faciment après un pédiluve prolongé.

Comme vous le voyez, l'Ail se rapproche par ses opriétés des Crucifères, dont il exagère pour ainsi re les vertus médicales. Quant à l'inconvénient 3 son usage à cause de son odeur, c'est une queson de peu d'importance dans les campagnes et i ne devrait jamais arrêter, dans les villes, lorsu son emploi est indiqué. On peut d'ailleurs neualiser sensiblement son odeur en mâchant des 
feuilles de persil ou de cerfeuil. L'Ognon (Alliv сера) réunit, mais à un degré moins développé, s propriétés précieuses de l'Ail.

Maintenant que vous avez tous fait rotre cuclette, nous allons nous asseoir à l'ombre, sur $\theta$ beau gazon, avec le ciel bleu sur nos têtes, écctant les gazouillements et les chansons du ruisser, des pinsons et des fauvettes. C'est dans un milia comme celui-ci que l'on retire le plus de profit $\theta$ l'étude de la nature; l'attention que nous donncs aux détails est rendue plus fructueuse par les i pressions de l'ensemble.

Je suis obligé d'éliminer bien des plantes util, parmi celles que vous avez récoltées, mais le ters que nous pouvons dédier à ces leçons ne nous $\mathrm{p}$ met pas de nous occuper des remèdes d'une impitance secondaire ou d'énumérer tous les végétax qui peuvent remplir le même but. D'ailleurs, cherchant à rendre notre étude plus complètel me faudrait surcharger votre mémoire, et courire risque de vous voir tout oublier pour avoir voru trop apprendre.

J'ai mis à part trois plantes de la même famil, celle des Composées qui nous a déjà fourni l'Arone et le Pissenlit.

Voici d'abord l'Armorse (Artemisia vulgaris) u Herbe de la Saint-Jean, que vous reconnaitre:à ses feuilles décomposées d'un vert sombre en dsus, blanches et cotonneuses en dessous, qui viennent linéaires à l'extrémité des rameaux. Cel:ci portent une panicule longue et étroite de fleis 
sposées en épis axillaires, c'est-à-dire attachés à xe princ:pal. Ces fleurs d'un jaune verdâtre en rme de capitule de forme ovoide s'ouvrent en ai-juin. C'est une plante vivace, haute d'un mètre plus, que l'on rencontre dans les terrains incul3 , les ruines, et qui se plaît dans les lieux arides. Ses propriétés se rapprochent de celles de l'Abthe, mais elles sont moins énergiques; elle est nique, stimulante, antispasmodique et emmenague, c'est-à-dire capable de rappeler les écouleents naturels supprimés, l'odeur des sommités $t$ aromatique, leur saveur un peu amère. On reteille les sommités au moment de la flcraison et 1 les sèche avec soin. Elles servent à préparer avec - l'eau ou du vin une infusion dans laquelle on nploie de 10 à 30 grammes de la plante par litre liquide.

Ce que je viens de vous dire s'applique à la MAIGAIRE (Matricaria parthenium), qui montre un u plus tard ses fleurs assez semblables à de petes pâquerettes, disposées en corymbes à l'extréité des rameaux, nommée vulgairement Esparjutte, Eil de soleil; et aussi à la Matricaire Momille (Matricaria chamomilla) dont le feuilge se rapproche de celui de l'Armoise, mais dont s fleurs, plus grandes que celles de la Matricaire, montrent isolées à l'extrémité des rameaux.

Vous avez remarqué sans doute, dans les lieux ca, sablonneux, le long des routes, cette plante à ges étalées, demi-couchées, hautes de 30 à 35 centiiètres, aux feuilles composées de nombreuses désupures linéaires, courtes, un peu velues, aux 
fleurs un peu plus petites que celles de la $\Lambda$ tr caire : c'est la Camomille romaine (Anthemis :bi lis) dont les propriétés sont malheureusemet e raison inverse de la beauté des fleurs quel'o double par la culture. On récolte celles-ci de j lle à septembre, un peu avant leur entier épanoisst ment, et on les sèche à l'étuve ou au soleil. n faut pas la confondre avec la Camomille PINT (Anthemis cotula) ou Maroute, Bouillot, Arou roche, qui d'ailleurs, à part son odeur désagrésle s'en rapproche beaucoup par ses propriétés, et qu est fort utile contre les accidents nerveux.

Comme les plantes dont nous venons de paer la Camomille est tonique, stimulante, fébrilge anthelmintique, antispasmodique. Il est util d remarquer que la décoction est particulièrelen tonique, tandis que l'infusion, plus chargé di principes volatils, est spécialement excitant e antispasmodique. L'une et l'autre se préparent vel 4 à 8 grammes de fleurs par litre d'eau. L'infuior est fort utile dans les coliques venteuses, les ;cidents nerveux, l'affaiblissement qui accomp nt la fièvre typhoìde et aussi contre quelques néral. gies; elle favorise l'action des vomitifs. Les bins de Camomille rendent de grands services auxnfants débiles, scrofuleux.

L'infusion concentrée (8 à 15 grammes par tre d'eau), ou la poudre des fleurs à la dose de à 8 grammes dans du vin ou en pilules constituen excellent fébrifuge, soit qu'on l'administre sele, soit qu'on lui adjoigne d'autres remèdes de cte classe, amers ou astringents, comme la petite $\mathrm{C}$ taurée, la Benoite, l'Ecorce de saule. Mais n'lblions pas, à propos des fièvres d'accès ou fières 


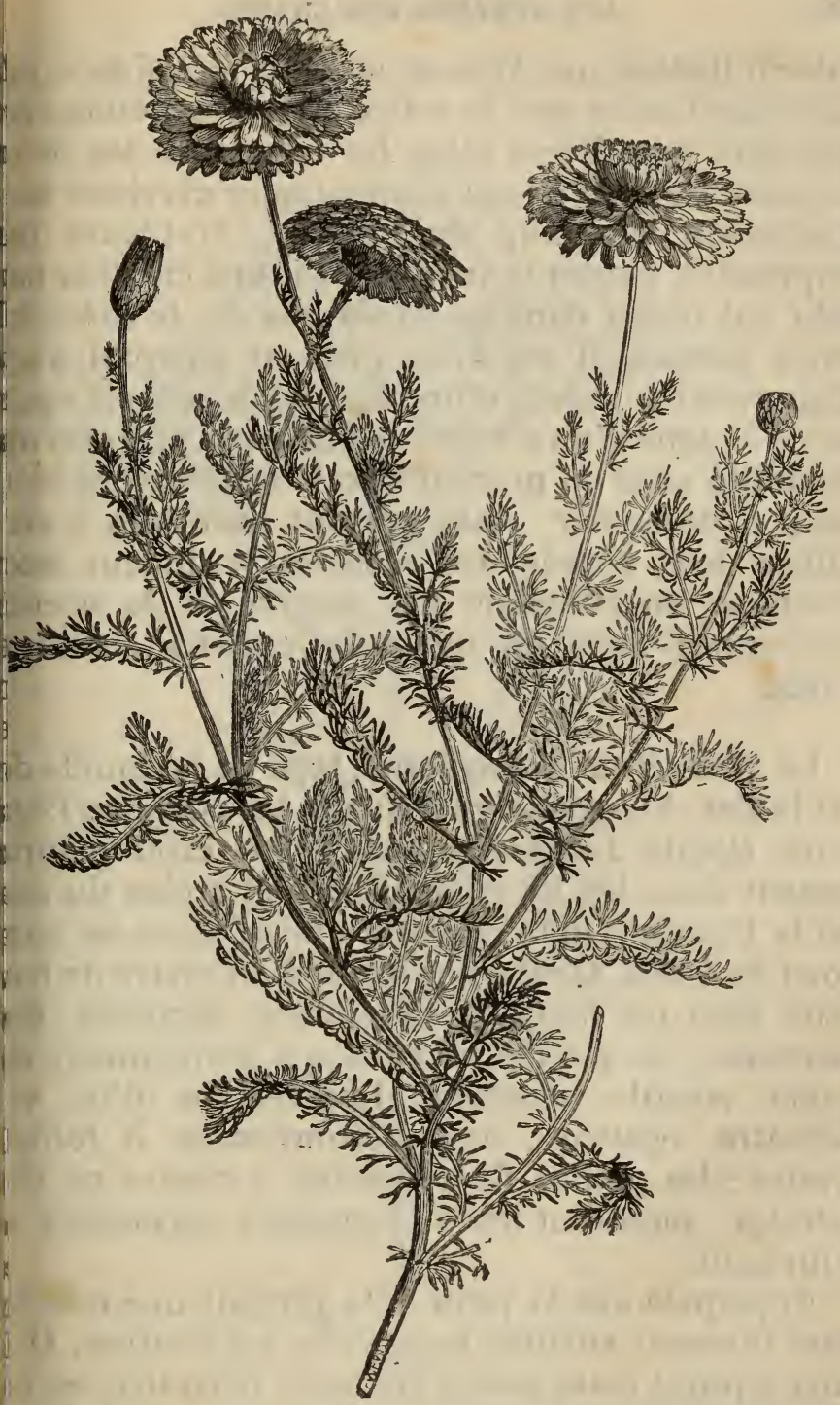

Camomille cultivée. 
intermittentes, que l'on est souvent obligé de varyr la médication et que le même traitement donne $: s$ résultats très-divers selon les individus, les lie: les saisons. C'est ce qui a fait adopter et rejeter sis fondement beaucoup de remèdes. D'ailleurs ie impression physique très-vive ou une émotion $n_{1-}$ rale ont réussi dans quelques cas où la médeco avait échoué. Il est donc prudent lorsqu'il s'ait d'apprécier la valeur d'un remède, de se tenir ég:ment éloigné d'un scepticisme de parti pris et d'ı crédulité amie du merveilleux. Il reste encore belcoup à découvrir, mais surtout beaucoup à si-plifier dans l'étude des remèdes et de leur mie d'action; en attendant les progrès de la scien; contentons-nous de quelques notions saines t sûres.

La Rue (Ruta graveolens), type de la famille is Rutacées, à laquelle on a donné les noms de Pé:nion, Rouda, Ruda, Herbe de grâce, croît natuilement dans les lieux montueux et arides du mli de la France. On la rencontre aussi dans les enrons de Paris. C'est un arbuste d'un mètre de $\mathrm{h}_{\mathrm{i}}$ teur dont les rameaux supérieurs, herbacés, sit parsemés de petites glandes qui contiennent te huile volatile odorante. Les feuilles d'un v't bleuâtre, épaisses, sont décomposées, à folios ovales; les petites fleurs jaunes, à quatre ou ciq pétales. montrent leurs corymbes terminaux n juin-août.

Appliquée sur la peau, elle produit une rubéftion intense, surtout lorsqu'elie est contuse. O」a mis à profit cette action irritante dérivative en $\mathrm{f}$ sant porter sur la poitrine un linge imprégné de $s$. 
ou trempé dans sa décoction concentrée, pour

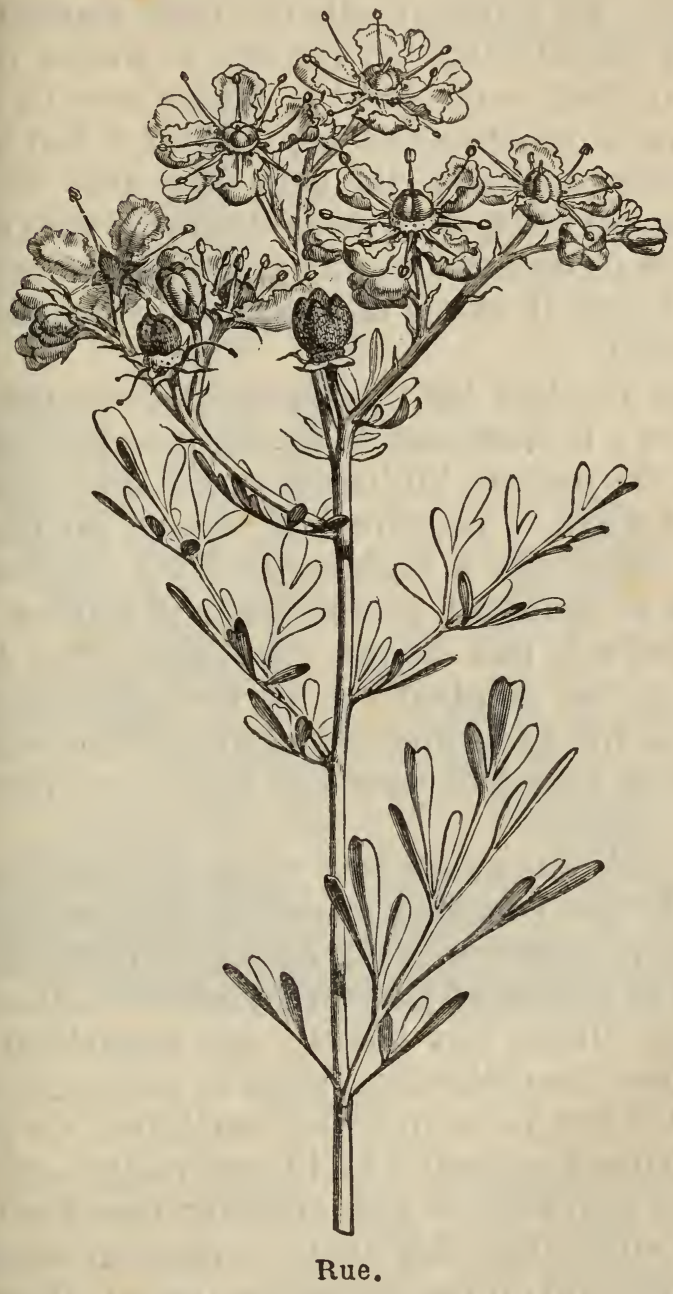

érir des catarrhes chroniques. C'est un an ti-paraaire fort utile en lotions contre les poux, la gale, 
la teigne, les larves de mouches qui se trovent parfois dans les plaies ; et pour déterger les ul;re atoniques. En lavement elle fait périr prompte en les vers intestinaux. On obtient le même réslta chez les enfants en frottant le ventre avec de l'xil d'olive ou d'œillette dans laquelle on a fait in se des feuilles de Rue; elle peut être utile dan le accidents nerveux, et possède des propriétés enaé. nagogues incontestables, mais dont il faut se gade: d'user lorsqu'il existe une cause quelconque in flammation.

Il faut récolter les tigès avant l'épanouisser:n des fleurs; la dessiccation ne diminue pas ses 0. priétés. On prépare l'infusion avec 2 à 10 gramies par litre d'eau, à prendre par tasses; on donr lí poudre en pilules à la dose de $1 / 2$ à 3 gramıs. La Rue se sème au printemps, sur couche, oren pleine terre à une bonne exposition. Ses toves restent vertes pendant les hivers peu rigourex mais si le froid et l'humidité font périr les rameix et les tiges, la racine reproduit une plante nouvee.

J'ai appelé plusieurs fois votre attention sures plantes à tige couchée, traçante, d'où partent s1vent des racines; nous en avons un exemple d is celle-ci, la VÉronique (Veronica officinalis) ou '] ié d'Europe, Herbe aux ladres, qui appartient à a famille des Scrofulariées. Vous la reconnaitrez cilement à ses jolies grappes axillaires de petis fleurs bleues, longues de 8 à 12 centimètres, qui flerissent en juin-août, et à ses fruits en forme de cœv. On la récolte dans les lieux arides qu'elle afftionne, pendant ou peu de temps après la floraiso; on emploie en infusion les feuilles et les sommily 
sont légèrement toniques et excitantes, utiles s les catarrhes pulmonaires chroniques, les flasités, la dyspepsie. Quant aux autres vertus de Téronique, elles ne sont nullement prouvées. Il

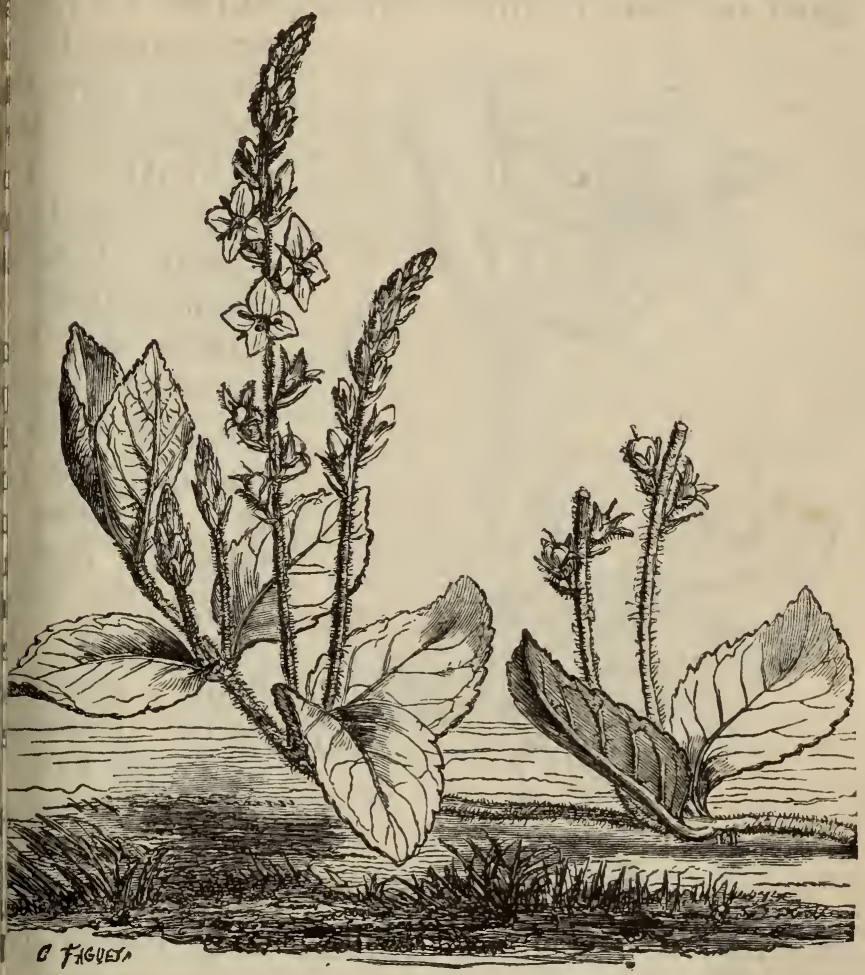

Véronique.

te plusieurs variétés qui peuvent remplacer e-ci, ce sont: la VÉRONIQUE PETTT GHÊNe, remarble par les deux rangées de poils des rameaux, téronique a ́́pi et la Véronique Germandrée, a 
fleurs plus grandes, commune dans les bois, ll amère que les autres espèces.

La plante vivace que vous voyez ici, croît paot dans les lieux incultes, sur les ruines, les rocer

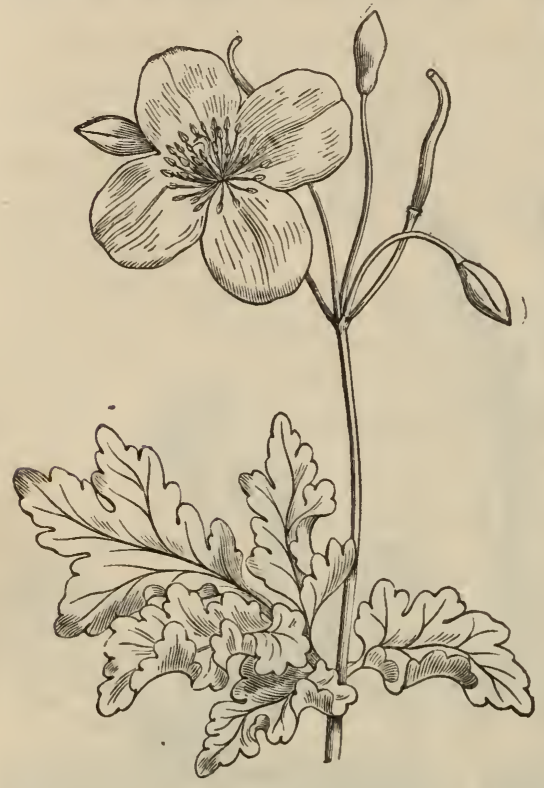

Chélidoine. on l'appelle Ecim Herbe d'hiron 11 , Felougène, Irk dentaire. Jou nous, c'est la LIDorne (Chede nium majus), famille des $\boldsymbol{I} p$ veracées, sur 1 quelle je vous or nerai, tout à liee re, quelques di tails. La racif e. forme de fular chevelue, brun rougere donne naissare une tige hauf $d$ 4 à 7 décimèr fragile, garni d: longs poils, poar des feuilles alternes, molles, à segments olle crénelés, et terminée par une ombelle de cinq si petites fleurs jaunes à quatre pétales qui se un trent d'avril à septembre, et sont remplacéespa des fruits en forme de silique.

Toutes les parties de la Chélidoine sont ut's on la récolte de préférence un peu avant la $\mathrm{fl}$ a son. A l'état frais elle a une odeur désagriblr 
euf couvé. Sa tige renferme un suc jaune, caus[ue, âcre et amer qui contient de la gomme-gutte. dessiccation diminue son âcreté et augmento n amertume. La racine est plus active que la inte.

A la dose de 60 à 90 grammes, le suc frais de élidoine tue un chien de taille moyenne. C'est un ison narcotico-âcre qui agit d'abord en irritant les ganes de la digestion, puis en produisant le nartisme ou assoupissement accompagné de délire, iallucinations.

L'activité de cette plante, employée avec précaun, la rend fort utile dans les engorgements, l'hyopisie, les scrofules, la goutte, les dartres. Elle i excitante, diurétique, purgative et vomitive;

e possède en outre une vertu altérante qui la ad précieuse dans beaucoup de maladies chroyues. On donne, dans les cas que je viens de er, 5 à 6 grammes de suc frais dans 700 gram. is environ de petit lait, à prendre chaque jour en sieurs doses. Comme purgatif ou vermifuge, on ble le suc à du jaune d'oeuf, du mucilage de Guiauve ou de graine de Lin; la quantité varie de elques gouttes à une cuillerée à café; cette derire dose agit souvent comme éméto-cathartique. suc étendu d'eau forme une lotion détersive et citante pour les ulcères de mauvaise nature; il ut rendre aussi des services comme anti-parasire; quant à son emploi contre les verrues, il est u efficace et produit l'inflammation des parties isines.

Lorsqu'on ne peut pas employer le suc frais, on ministre l'infusion ou la décoction de plante sèche a dose 15 à 30 grammes par litre d'eau, à pren- 
dre par tasses dans les vingt-quatre heures. Si $1 \mathrm{n}$ emploie la racine, on diminue la dose de près $\theta$ moitié; on peut aussi préparer par infusion, un ' ou une bière de Chélidoine. Son nom d'Eclaire vi t

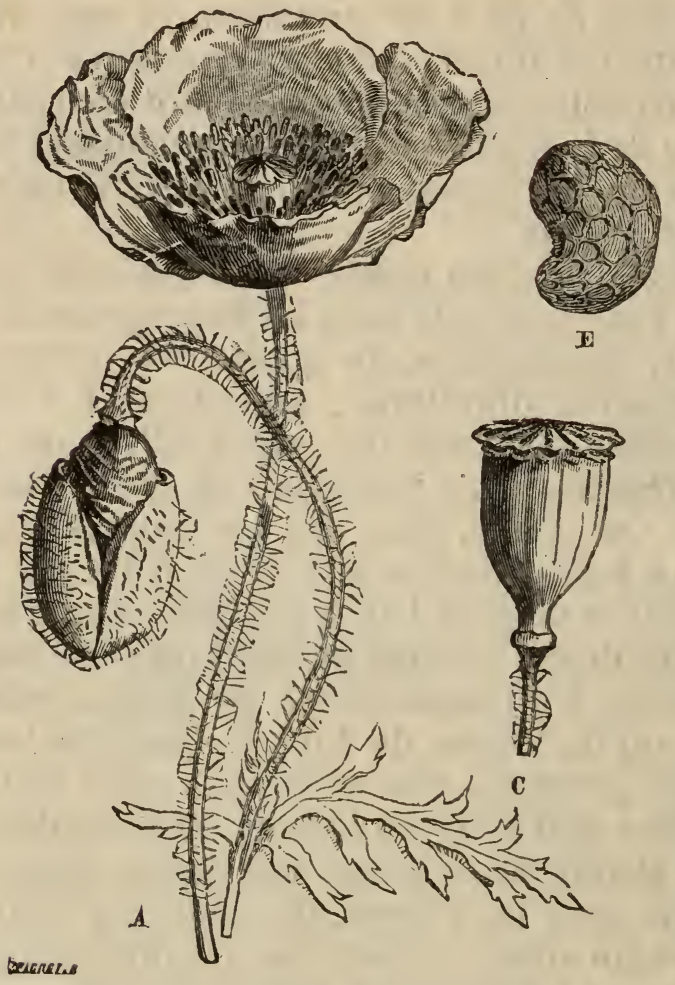

Coquelicot. - A, fleur et bouton. - C, fruit. - E, graine.

de l'usage populaire que l'on en fait, non sans su cès, dans les campagnes pour la guérison des op thalmies chroniques. On emploie dans ce cas, i collyre composé de $4 \mathrm{gr}$. de suc étendu dans 60 . 
10 gr. d'eau, dans lequel on baigne les yeux une deux fois par jour.

Je viens de vous annoncer quelques détails sur famille des Papavéracées. En voici un type famir, c'est le Coquelicot (Papaver rhoeas) ou Ponau, Mahon, Pavot des champs. On reconnait les antes de cette famille au calice formé de deux pales, à la corolle composée de quatre grands tales, renfermant de très-nombreuses étamines un ovaire qui plus tard devient, dans presque utes les espèces, une capsule à nombreux comrtiments surmontée d'une espèce de collerette. is feuilles, semi-composées, dentées et pileuses, nt profondément incisées.

Le Coquelicot, ornement de nos champs où il se altiplie parfois outre mesure, fournit à la méde1e ses pétales dont on emploie l'infusion ( 3 à 4 cées par litre d'eau) à titre de calmant dans le arrhe pulmonaire, la coqucluche, les coliques 13 enfants. La décoction des capsules est plus ergique, et peut dans quelques cas remplacer antageusement l'opium.

Dans les jardins on sème le Coquelicot au prinInps, ou mieux à l'automne, dans un sol bien liendé; la cultưre produit des variétés blanches lettes, panachées et semi-doubles, qui se reproisent par semis.

Jn a donné à la petite plante que voici les noms Surelle, Pain de coucou, Oseille de Pâques, Trèfle re : c'est l'Alléluia ou Oxalide (Oxalis aceto- 
sella), de la famille des Oxalidacées. Remarq 32 son rhizôme écailleux, traçant, d'où partent dir tement de petites feuilles semblables à celles trèfle, pileuses et blanchâtres en dessous, et petites fleurs blanches solitaires sur un long péd. cule. C'est une plante vivace, abondante dans lieux ombragés. Ses feuilles fraiches ont une savir acide agréable : on en prépare une boisson rafichissante, tempérante, diurétique, antiscorbutiqg, qui remplace très-bien la limonade dans les min dies inflammatoires, putrides, les fièvres maligı certains désordres de la digestion. Elles doivnt leurs propriétés à un oxalate de potasse (sel seille) employé en teinture et dont on se sert p enlever les taches d'encre. L'extraction de ce se fait en Suisse et en Allemagne.

Appliquées contuses sur des tumeurs scrcileuses, des abcès froids, les feuilles d'Alléluia ą̧ sent comme maturatif.

Tout ce que je viens de vous dire au sujet l'Alléluia s'appliquo à l'Oseille sauvage ou Sure et à l'Oseille commune (Rumex acetosa) culti? dans les jardins potagers.

Il y a, mes amis, des végétaux qui semblent do de qualités sociales par rapport à l'homme, l'accompagnent partout, et semblent croître spl tanément sous ses pas. Celle-ci en est un exemle frappant. En Europe, en Asie, en Amérique, sis les climats tempérés commo sous l'équateur, $\mathrm{r}$ tout où l'homme a construit une cabane, même u sein de forêts vierges, on ne tarde pas à voir cite plante amie, la MaUve (Nialva sylvestris), type dila famille des Malvacées. Dans ses fleurs que l'on (1- 
ploie en tisane, dans ses feuilles dont on fait is cataplasmes, dans ses racines riches en mucilas réside la même vertu émolliente, adoucissante, uto dans les maladies aiguës toutes les fois qu'il ja inflammation. L'infusion des fleurs (10 à $15 \mathrm{gra}$ mes par litre d'eau) est la préparation la plus usit On recueille les fleurs et les feuilles au commenment de l'été : la racine, moins employée, n't guère usitée qu'à l'état frais. La Gunauve ( $A l t l_{2}$ officinalis) a des propriétés identiques.

Disons en passant que le Cotonnier, le roi e l'industrie, comme l'appellent les Américains, :partient à la même famillo que la Mauve.

Il faudrait écrire un livre pour relater toutes propriétés de la plante que vous voyez ici, travaux auxquels elles ont donné lieu, les ex riences des médecins au sujet de cet agent précios et redoutable en même temps. Je vais essayer né moins de vous le faire connaitre, dans la mes!e que comporte la nature de nos entretiens :

La BeLladone (Atropa belladona) appartient àa famille des Solanées qui nous a déjà fourni come sujets d'étude la Morelle, la Pomme de terre, Coqueret, la Douce-amère. Les noms vulgraires $\theta$ lui font pas défaut; on la nomme : Belle-dar Morelle furieuse, Mandragore baccifère, Parm ton, Guigne de côte, Herbe empoisonnée. Elle cit dans les lieux ombragés, le long des haies, murs. La racine vivace est épaisse, longue et meuse; la tige, un peu velue, porte d'assez gran feuilles ovales-aiguës entières, molles, d'un sombre, et de leur aisselle partent des fleurs d pourpre obscur, solitaires ou deux à deux, form 


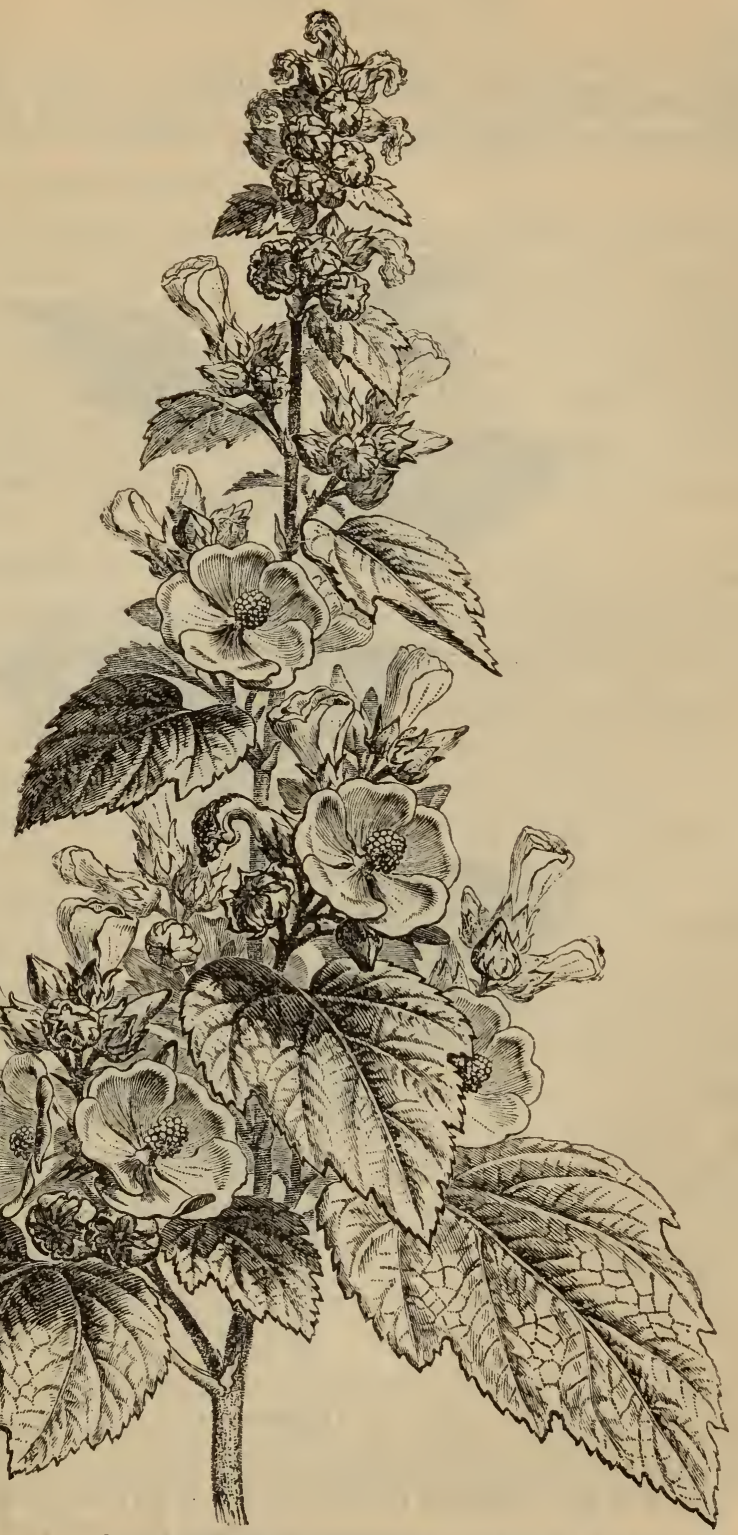

Sommité de la Guimauze. 
d'une corolle en cloche allongée à cinq divisi obtuses auxquelles succèdent des baies de la g15.

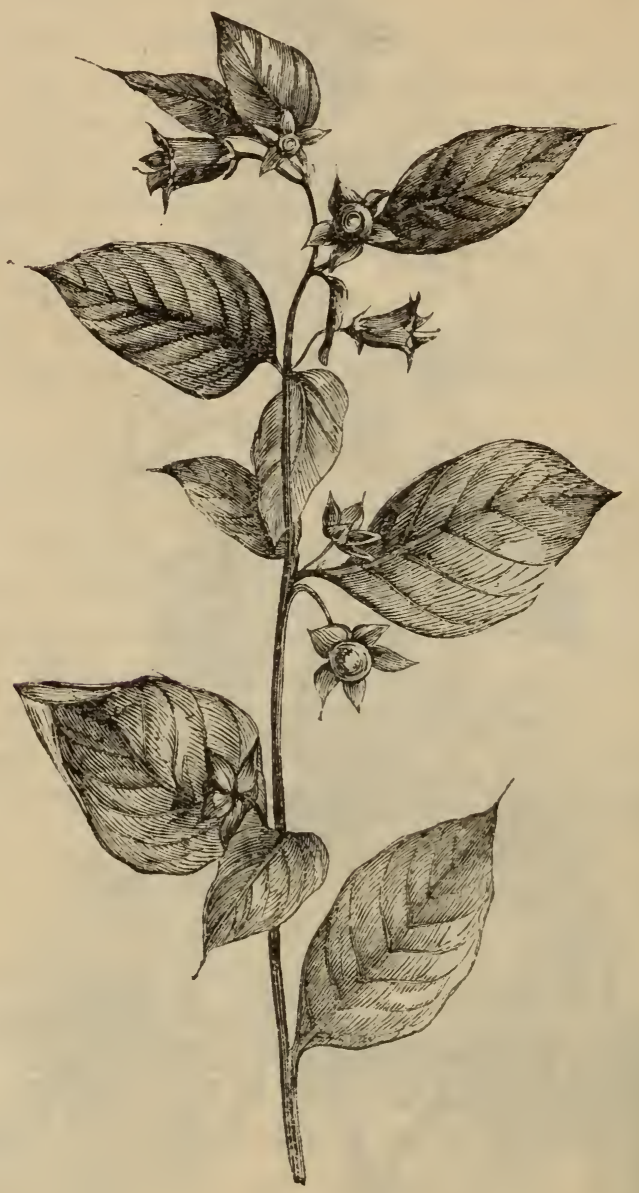

Belladone.

seur d'une petite cerise, d'abord vertes, puis roly et enfin noires, accompagnées du calice en éto 
inq divisions, qui ont causé de nombreux emoisonnements. Elle fleurit de juin à septembre; on écolte les feuilles en juin, les baies en août, la acine en mai et juin.

Toute la plante est douée d'une odeur vireuse et 'une saveur un peu âcre et nauséabonde. C'est un oison narcotico-âcre qui cause d'abord une excitaon générale, des nausées, puis une véritable folie mulant l'ivresse, un délire furieux, des spasmes t la mort. Cependant elle est recherchée par les uins, les moutons, les cochons, et ne leur fait auin mal.

La Belladone semble être le remède par excelince des névralgies. Lorsque l'affection est supercielle, il suffit d'appliquer sur le siége de la douur un cataplasme de feuilles fraîches contuses ou ieux de racine écrasée; dans les autres cas, on onne par jour, à la dose de 30 à 60 grammes, avec récaution et progressivement, l'infusion préparée vec 2 à 3 grammes de feuilles par litre d'eau. C'est n remède excellent pour calmer la douleur, émous'r la sensibilité, prévenir ou arrêter les convulsions, s spasmes qui accompagnent les crises nerveuses 1 le tétanos. Il compte de nombreux succès dans les liques sèches, les vomissements nerveux, la toux, s palpitations, l'asthme, l'angine de poitrine, les instrictions spasmodiques, les hernies étranglées, ncontinence d'urine. Entre les mains des ocustes, il rend de grands services pour dilater la upille et contribue, par ses vertus sédatives, à la uérison des ophthalmies.

Dans la coqueluche, la Belladone est peut-être le mède sur lequel on peut le plus compter. Austôt que la période catarrhale et inflammatoire est 
dissipée, on adıninistre de quatre heures en quat, heures de 2 à 5 centigrammes de poudre de racir, augmentant, suivant l'âge et les circonstances, ju. qu'à 25 centigrammes. Co traitement qui modi? promptement les quintes, amène souvent la gi. rison au bout de trois ou quatre septennaires. En. il semble prouvé que l'usage de petites doses Belladone, deux ou trois gouttes de la teinturo po des enfants de deux à quatre ans, préserve de scarlatine.

Il arrive four ce médicament, comme pour bec coup d'autres, que l'on n'en retire pas tout le bi possible, ou même que l'on échoue dans son e: ploi, faute d'en continuer assez longtemps l'usae ou d'arriver à des doses assez fortes. Lorsque symptômes à combattre sont graves, il est nécrsaire d'augmenter ou de rapprocher suffisammit les doses pour produire ce que l'on appelle l'eft physiologique du médicament, c'est-à-dire les tri bles qui précèdent les accidents auxquels des qui tités trop fortes pourraient donner lieu.

Ce que je viens de vous dire au sujet de la Beldone peut s'appliquer, en grande partie, à une aut Solanée dont nous avons cueilli un exemplaire, Jusquiane (Hyoscyamus niger) ou Hidnebare, Polie, Herbe aux engelures, Herbe à la teigne, $P_{1}$. celet, Mort-aux-poules; plante bisannuelle tr commune près des lieux habités et qui, malgré s propriétés toxiques pour l'hommo, ne cause auca accident aux chèvres, aux vaches, aux cocho, aux brebis.

La racine épaisse et ridée, brune en dehors, bla che on dedans, a quelquefois été prise pour celle 
corée. La tige velue, haute de 50 à 60 centimè. , est garnie de longues feuilles cotonneuses, lan-

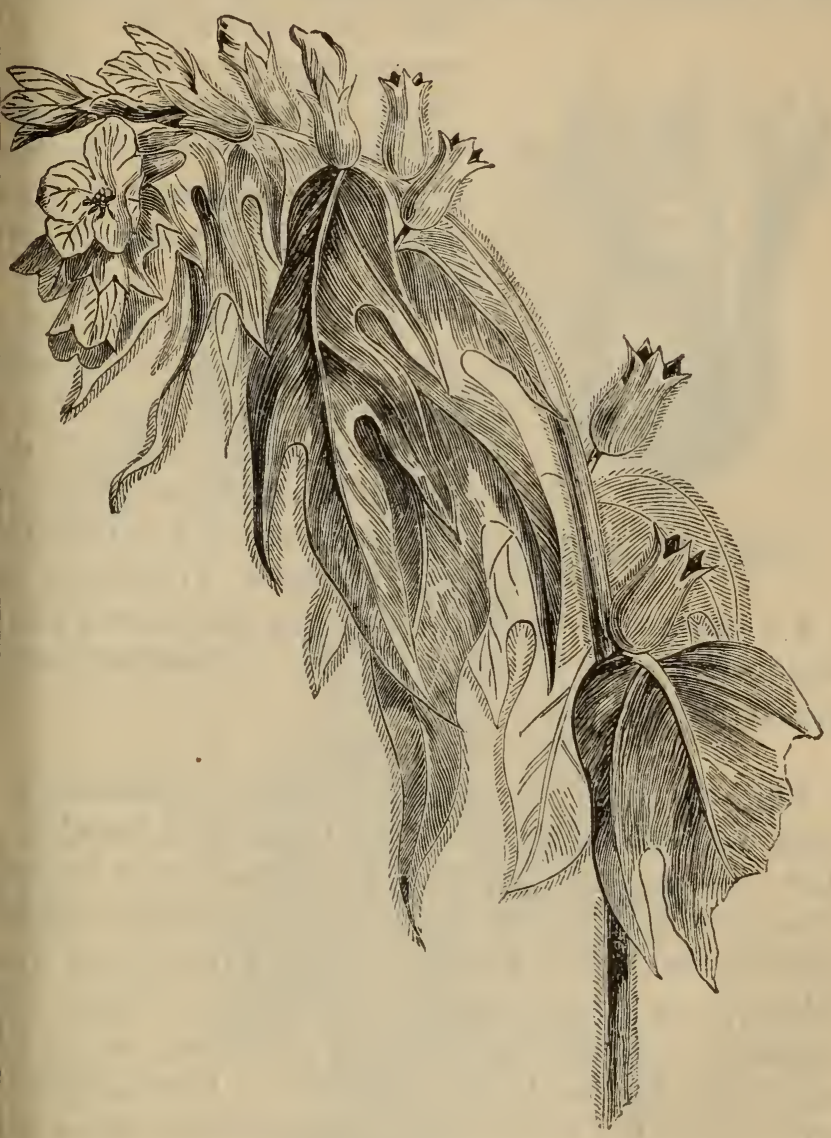

Jusquiaue.

ées, profondément découpées; les fleurs camulées, d'un brun jaunâtre, veinées et marquées pourpre, forment d'un seul côté des sommités 
feuillées un long épi recourbé; elles paraissen de mai à juillet et sont remplacées par une capsu deux compartiments qui su.

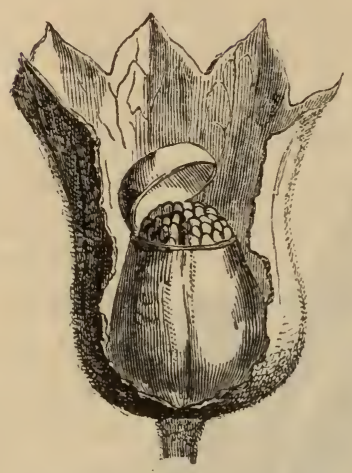

Capsule de Jusquiame. vre comme une boîte à sa'n nette, pour laisser échares les graines mûres. On réct la plante comme la pré dente, un peu avant la flcii son.

La Jusquiame est un son narcotico-âcre qui affti spécialement le système veux, et produit, mais à d e plus élevées, des effets ta logues à ceux de la Belladie On prépare l'infusion ie 2 à 4 grammes de feuilles sèches par litre d'eauol donne le suc à la dose de 1 à 4 grammes et 10 gressivement.

J'ai mis de côté pendant notre promenade e trois plantes vénéneuses de la même famille, que vous soyez à méme de les examiner compa tivement et de les distinguer d'autres végétus inoffensifs. Voici d'abord la CIGü̈ (Conium mil. latum), ou Ciguë commune, Grande Ciguë, Ciı tachetée, qui est le type de ce genre, et la sil employée en médecine. C'est une Ombellifère croît dans les lieux frais, les terrains gras etn. cultes. La racine bisannuelle, blanche, pivotaie grosse comme le petit doigt, est longue de : 25 centimètres. La tige épaisse, fistuleuse, un $u$ striée, d'un vert clair tacheté de pourpre violacuy de brun noirâtre, atteint de 1 à 2 mètres de 1 A 
Ir ; elle contient un suc blanc. Les feuilles alter$s$ grandes, un peu molles, sont décomposées

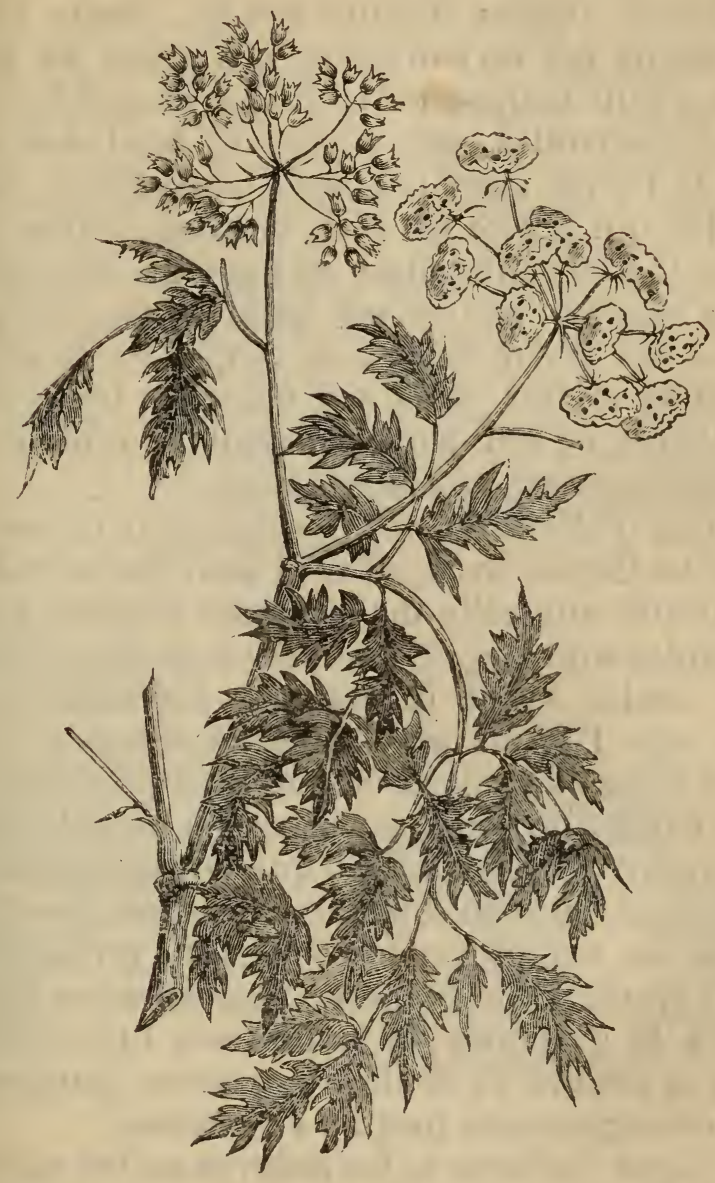

Ciguë.

leurs folioles aiguës, incisées, ressemblent assez celles du Persil sauvage. Les fleurs blanches, très- 
petites, forment des ombelles terminales de 10 12 rayons; elles paraissent en juin-août. La plat répand une odeur fétide.

La Ciguë vineuse (Cicuta virosa), plante viva se distingue par un suc jaune, une odeur de pers une tige sans taches, un feuillage plus délié à lioles très-étroites, elle habite le bord des eat Enfin la Petrte cigü̈ (AEthusa cynapium), plar annuelle, qui habite les lieux cultivés, n'attei:it $\mathrm{f}$ plus de 45 à 60 centimètres de hauteur, elle contic un suc incolore, sa tige est tachetée de violet à base, et son odeur vireuse se rapproche de celle la Grande ciguë, mais ses ombelles très-garni, sont planes, et son feuillage tient le miiteu ent ceux des deux espèces précédentes.

Un peu d'attention suffira donc pour re pas ec fondre les Ciguës avec le Persil dont l'odeur est pl aromatique que celle de la Ciguë vireuse, et dc les folioles sont larges, trilobées et en forme de coi

On récolte avant la floraison les sommités Ciguë, que l'on sèche à l'abri de la lumière. M il vaut beaucoup micux employer la plante fraîcl ou un extrait de son suc préparé à une très-bas température. Cette plante, qui constitue dans pays chauds un poison narcotico-âcre, perd ral dement de ses propriétés à mesure qu'on avan vers le Nord. Pour préparer l'infusion, on emple de 20 à 40 grammes' de feuilles par litre d'eau; donne la poudre de feuilles par doses progressiv de 10 centigrammes jusqu'à 4 grammes.

La Ciguë n'affecte ni les chèvres ni les moutor mais elle empoisonne les lapins, les bœufs, les ch vaux. Chez l'homme, les doses toxiques produise la soif, les vomissements, des douleurs de tête 
las feuilles et les racines sont diurétiques et lég: ment astringentes, tandis que ses fruits sont ra chissants et tempérants.

Cette plante nous offre un exemple, plus re:ai quable que la Pervenche et la Véronique, de taux chez lesquels il semble qu'il n'y a pas dei proprement dite. Les racines, à peu près de nn taille, partent d'un même point sans dépendre axe plus considérable; au-dessus s'élève une ro de feuilles qui couvre la terre. Mais il n'y a pi feuilles sans tige, et nous devons admettre à ce l'axe qui se montre à la surface du sol. Cet axe d naissance à de véritables rameaux ou nouveaux nommés coulants munis d'appendices à l'ais desquels naissent de nouveaux bourgeons qu veloppent bientót des feuilles et des racines.

Vous me demandez, mes amis si l'Églain (Rosa canina) est bon à quelque chose. Il s
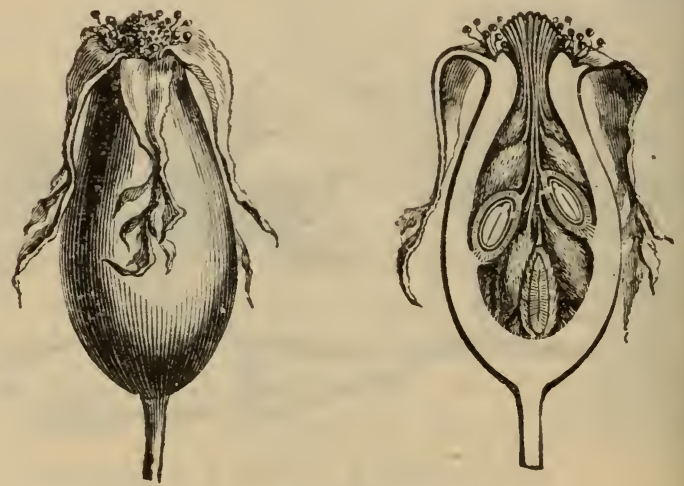

Fruit de l'Églantier.

vraiment regrettable que cet ornement de nos lii et de nos buissons n'eût d'autre attrait que cel 
fleurs rosées, jaunes ou rouges, selon les vaés. Vous avez reconnu déjà que c'est un membre

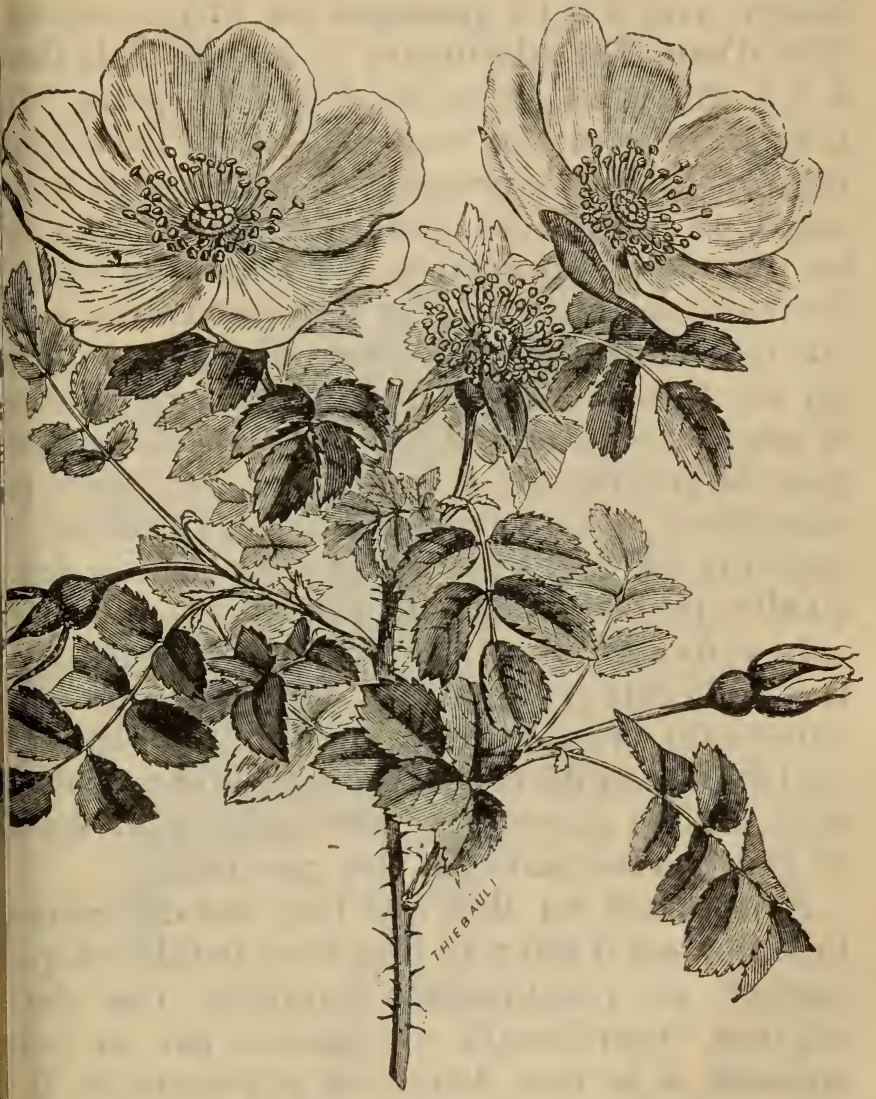

Eglantier.

i famille des Rosacées qui nous a fourni deux ts intéressants : le Pommier et le Pêcher.

i médecine emploie surtout la Rose de Pro(Rosa Gallica) naturelle du midi de la France, 
qui est astringente et tonique à un faible deg convient aux personnes délicates. On prépare fusion avec 8 à 15 grammes de fleurs sèchessa litre d'eau. On administre la poudre à la dos 4 à 8 grammes, dans du miel, du jaune d'u L'églantier commun nous fournit ses fruits (cyp rhodons) dont on prépare, en les écrasant ave d sucre, un sirop astringent qui réussit bien ccth les diarrhées des enfants. On a soin de déporl les fruits des poils dont ils sont recouverts, à mit que l'on ne veuille produire un effet anthelmintiu car ces fines aiguilles, qui causent, applıquées la peau, une démangeaison insupportable accorp gnée de gonflement et de douleur, n'affectent $p$ muqueuse qui tapisse la bouche, l'estomac el intestins, et vont s'implanter dans la peau des qu'elles font promptement mourir.

Je vous citerai, en passant, une autre Rosace RoNce (Rubus fructicosus), dont les fruits peus fournir un sirop rafraîchissant, et dont les tig les feuilles, en décoction concentrée et miellée, nent un bon gargarisme astringent pour les $r$ de gorge et les maladies des gencives.

Nous avons vu déjà que l'un des caractèresil Rosacées est d'avoir au plus cinq pétales et qu l portent de nombreuses étamines. Ces dere organes, transformés en pétales par la cul donnent à la rose double sa physionomie. Il arriver aussi, que par suite d'un excédant de lité, l'ovaire devienne foliacé, et donne naissale un nouveau bouton qui s'épanouit au-dessus première fleur, c'est ainsi que l'homme a cr Rose prolifére.

De ces faits vous pouvez déduire que l'homn 
it pas se borner au rôle de spectateur curieux ou

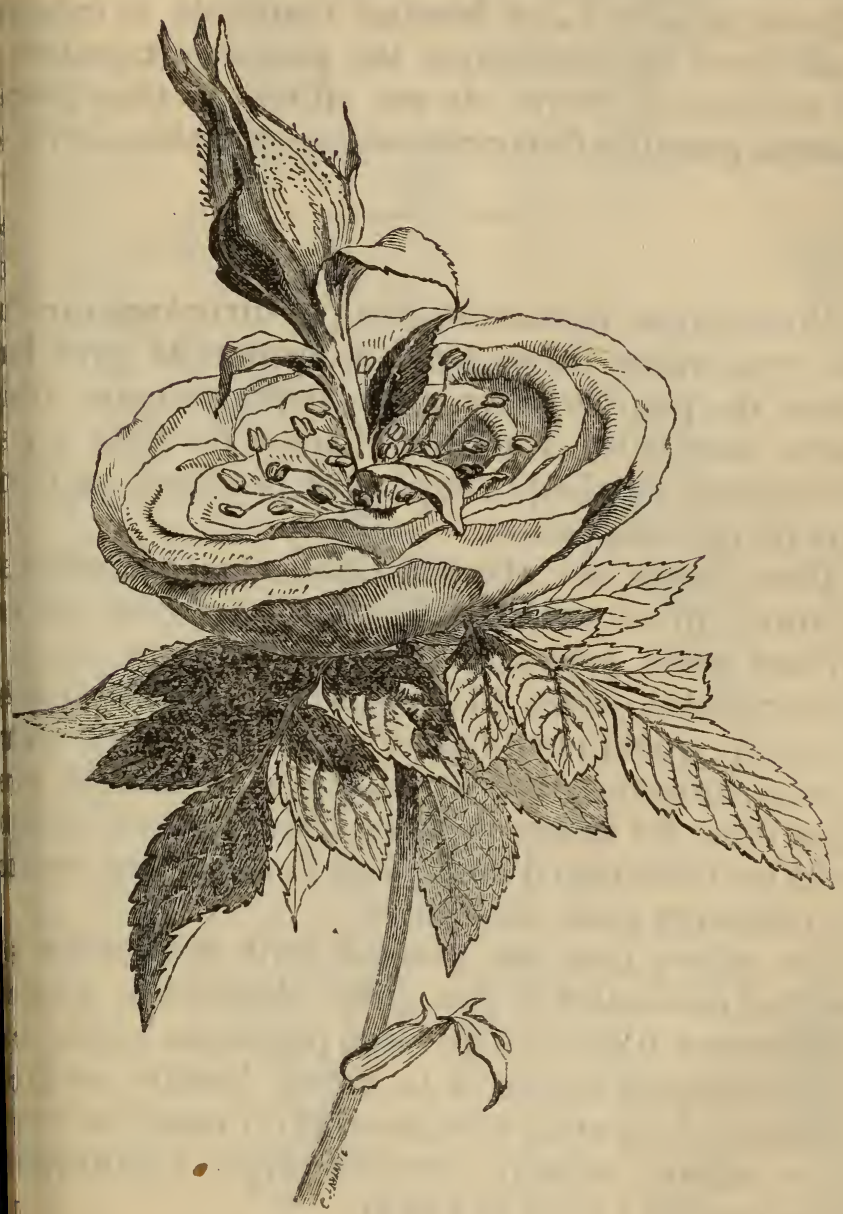

Rose prolifère.

1ousiaste de la nature, mais qu'il doit mettre à fit les leçons reçues d'abord du hasard, puis 2 PARTIE. 
complétées par l'expérimentation, pour cherchel bien connaître les lois qui régissent la matière bru afin de la plier à ses besoins matériels et intell. tuels dans les conditions les plus avantageuses de manière à retirer do ses efforts la plus grar sommo possible de connaissances et de bien-êtro.

Vous voyez, mes amis, que j'ai dirigé aujourd' vos recherches de manière à former de gros bi quets de plantes odorantes. Asseyons-nous, tri notre cueillette, afin que chacun de vous ait exemplaire de chaque espèce et préparez pour in une de ces collections.

Dans toutes ces plantes, nous n'avons guère, $n$ résumé, qu'un seul remède. Cela ne veut pas (' qu'une seule poitrrait, dans la pratique, les $r$ placer toutes, parce qu'elles offrent, dans leur m daction, quelques différences de détail, et que même substance ne produit pas les mêmes eft chez tcus les sujets et dans tous les cas, de sil quu'il est très-utile d'avoir sous la main, des remè de rechange pour ainsi dire.

De même que ces plantes sont douées de priétés médicales à peu près identiques, sauf différences d'énergie de leurs principes actifs, appartiennent toutes à la même famille, celle Labiées, dont nous connaissons un membre auc nous allons revenir commo typo botanique cette famille : c'est le Thym.

Chez les Labiées, plantes herbacées, presque jours vivaces, la tige et les rameaux de forme rée portent des feuilles simples, opposées, ovi: 
aiguës, c'est-à-dire chez lesquelles une extrémit de l'ovale se transforme en pointe, dentées et d surface ridée. Les fleurs disposées en petites cîme superposées, pressées à l'aisselle des feuilles supé rieures, sontirrégulières et renferment les organe reproducteurs mâle et femelle. Le calice est mo nosépale ou d'une seule pièce; la corolle est mo nopétale, c'est-à-dire d'une seule pièce aussi, mais c pétale unique est fendu profondément de manièr à former comme deux lèvres, d'où le nom de La biée (du latin labium, lèvre). Les étamines sont a nombre de quatre, deux petites et deux grandes. L pistil est fendu à la partie supérieure de manière y former deux stigmates; le style penètre dans $u$ ovaire qui présente, à la partie supérieure, quatr bosses qui correspondent à quatre loges renfermar. chacune un germe ou ovule. Une fois mûres, ce loges ou segments du fruit s'appellent des akèner

Maintenant que vous connaissez bien les carac tères que présentent les Labiées, vous serez à mêm de noter les différences de détails faciles à recon naître dans les diverses espèces.

Toutes les Labiées possèdent, à un degré plus o moins prononcé, des propriétés stimulantes, exci tantes, dues à un principe aromatique volatil 0 fixe. Quelques-unes contiennent, de plus, une sub: tance amère qui augmente l'effet et surtout la durt de leur action qui est alors excitante et tonique à fois. Laissez-moi fixer clairement dans votre espr le sens médical de ces deux mots.

Un remède excitant ou stimulant administré l'intérieur agit d'abord sur l'estomac dont il actir la sécrétion, où il détermine une sensation de chi leur ; puis, passant lans le sang, il accélère la circı 
ation, augmente la vitalité et cause une fièvre pasagère pendant laquelle toutes les fonctions se rouvent surexcitées. Quclques stimulants, outre ur action générale, semblent propres à concentrer avantage leur influence sur quelques organes, out en agissant d'une manière sensible sur toute économie. Mais l'excitation que procurent ces gents est bientôt suivie d'une ré iction pendant et orès laquelle les forces se retrouvent au même point u même plus bas qu'avant l'emploi de moyens arficiels d'excitation. Le propre des stimulants est onc de donner à la vitalité générale ou locale une lergie factice, temporaire. Un pareil résultat est rt utile dans une foule de cas oú l'on est obligé de urer au plus pressé, et dans beaucoup d'indisposions fugaces qui pas plus que la médication, ne issent de traces de leur passage.

Mais lorsqu'il s'agit de relever graduellement la talité et de la maintenir en cet état pour attendre développement, le déclin et la convalescence narelle des maladies, ce n'est plus aux stimulants 'il faut avoir recours d'une façon régulière, mais $\mathrm{x}$ toniques, spécialement aux amers et aux astrinints, dont l'action lente, mais persistante, rétablit ine manière permanente l'équilibre des fonctions (i constitue la santé.

l'avais besoin do vous donner au moins ces excations, pour vous mettre à même de juger dans felles circonstances et dans quelle mesure on est droit d'attendro des services de la classe de redes qui nous occupe.

'uisque vous voilà édifiés sur les généralités qui rapportent à l'usage médical des Labiées, il me 
suffira de vous faire une énumération rapide $d$ celles que nous avons recueillies, n'insistant qu sur les points les plus intéressants de leur histoire

Le Niarrube (Marrubium vulgare) ou Marroche min, Herbe vierge, croit partout dans les lieux in cultes; les fleurs, petites et blanches, se montrer de mai à octobre. On recueille, avant le développ ment des fleurs, les sommités, qui perdent par dessiccation leur odeur aromatique un peu musqué mais conservent leur saveur chaude, amère, nav séeuse et un peu âcre. 11 contient un principe a tringent et un peu de fer. Comme lo Lierre-terres tre, l'Hysope, il parait agir spécialement sur système pulmonaire, d'où son emploi dans le catas rhe chronique, l'asthme humide. la toux rebell C'est aussi un bon detersif des ulcères.

L'Agripaune (Leonorus cardiaca) ou encore $\mathrm{Ca}$ diaque, Cardiaire, Herbe aux tonneliers, affer tionne les alentours des habitations rurales, les te rains incultes. Elle atteint de 60 à 80 centimètres les feuilles sont palmées et pubescentes, les fleu un peu velues à l'cxtérieur, roses ou blanches, pon tuées de pourpre, s'ouvrent de juin à septembre. $C$ récolte avant et pendant la floraison les sommité d'une odeur aromatique peu agréable, d'une savet amère et âcre. On l'appelle vulgairement Cardiaqı parce qu'elle peut guérir les palpitations chez 1 enfants, lorsqu'elles n'ont pas d'autre cause que présence des vers qu'elle tue dans les intestins.

Lo Lierre-terrestre (Glecoma hederacea), vu gairement Glécome lierre, Couronne de terre, Ifer) de Saint-Jean, Rondette, Terretta, Drienne, se re 
ntre dans les endroits frais et ombragés, au pied s murs, des haies. Ses fleurs sont bleuâtres ou sées. On recueille les sommités en juin ou au mmencement de juillet. Son odeur est forte, aroatique; sa saveur, balsamique, amère et un peu re. Comme le Marrube, il semble exercer plus écialement son influence sur les orģanes de la spiration, et on l'emploie avec avantage dans les aladies chroniques de la poitrine.

Peu de plantes médicinales ont reçu autant de 1anges que la SAuge (Salvia officinalis), nommée ssi Sale, Herbe sacrée, Thé de la Grèce. C'est un us-arbrisseau que l'on trouve à l'état sauvage ns le midi de la France et que l'on cultive dans jardins. Elle demande un terrain léger et un peu aud. On peut cueillir pendant toute la belle sain ses feuilles épaisses, comme chagrinées, qui ne rdent pas par la dessiccation leur odeur aromatie, leur saveur chaude, piquante et un peu amère. trouve du camphre dans l'huile essentielle qu'elle ntient en assez grande quantité. Ello est utile ns les faiblesses d'estomac, les sueurs nocturnes, diarrhée des enfants débilités, les catarrhes roniques, les fièvres rhumatismales. C'est un bon tersif qui favorise la cicatrisation des blessures et s plaies suppurantes.

Voici une autre plante qui contient du camphre, st la Germandrée manitine (Teucrium marum), lgairement Marum, Herbe aux chats. Co dernier m lui vient de ce que les chats l'aiment autant o la Cataire et la Valériane, de sorte qu'on est ligé de la cultiver dans une cage grillée ou en 
pot suspendu. C'est un sous-arbrisseau aux tigs cotonneuses et blanches, qui croît naturellemes sur les bords de la Méditerranée. Les feuilles sor d'un vert grisître en dessus, duveteuses et blar ches en dessous; les fleurs purpurines, peu non breuses, un peu campanulées, se montrent en jui let-août. L'odeur de la Germandrée est balsamiqu très-pénétrante, sa saveur aromatique et très-amèr elle contient plusieurs principes astringents et ur huile essentielle camphrée. On peut lui substitu la Sauge, le Romarin, la Menthe poivrée, dont l propriétés sont analogues, et que l'on a toujou: sous la main. Il y a plusieurs variétés de Germar drée qui croissent spontanément dans nos départ ments du centre, mais leurs vertus sont assez fa bles.

La Menthe porvrée (Mentha piperita), or:ginai d'Angleterre, ce qui la fait nommer Menthe al glaise, est cultivée dans nos jardins. On récolte juillet, un peu avant la floraison, ses feuilles del tées en scie, d'un vert foncé en dessus, un peu di veteuses en dessous. Elle est remarquable par sc odeur vive, camphrée, balsamique, une saveı chaude, poivrée et camphrée qui laisse dans la bo che une sensation de froid caractéristique. El fournit une huile essentielle abondante dans li quelle résident ses propriétés de stimulant diffi sible, analogue, pour ses effets, à ceux de l'éther du camphre. On l'emploie avec succès dans les a fections que nous avons déja citées comme céda] à l'action des labiées, et aucuno ne semble aus efficice contre les désordres provenant d'atonio ( l'estomac, gastralgies ou dyspepsies sans inflan 
ation. Les autres variétés de Menthes nombreuses ns nos campagnes, utiles dans les mêmes cas, nt moins énergiques.

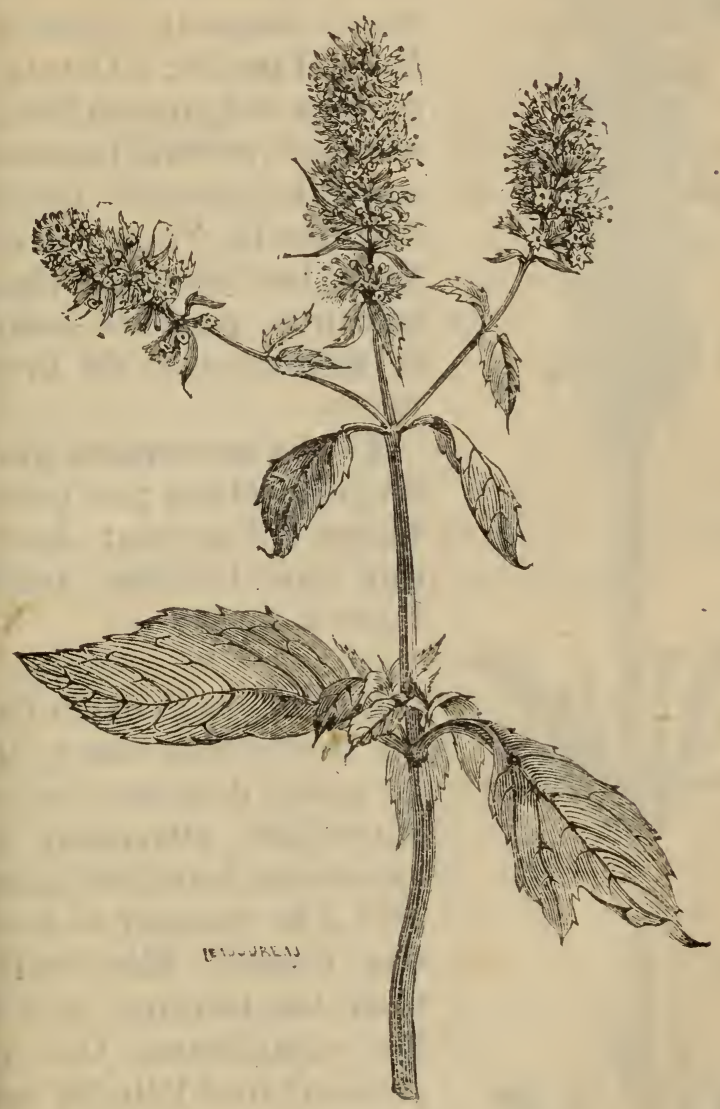

Menthe poivrée.

isons un seul groupo do ces quatre espèces ; les propriétés sont à peu près identiques : la IETTE (Satureia hortensis), ou Sadrée, Savou- 


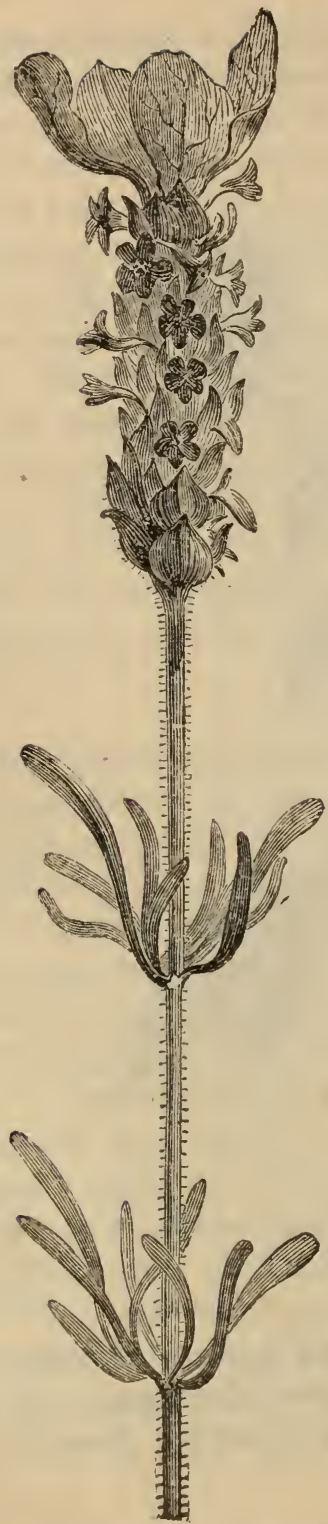

vée, Herbe de Saint-Juli lo Serpolet (Thymus seril lum), nommé aussi Thym :u. vage, Serpoisle, Pilolet, 0 . lour, Pouliet; l'Onigan (li ganum vulgare) ou Marjolan sauvage; enfin l'IIyssopé́l sopus officinalis) naturelit midi de ia France et cult é dans les terrains légerse calcaires, pour les besoin la médecine et de la pa merie.

Toutes ces espèces jouis:n de propriétés à peu près icn tiques et l'on peut, dans $p$ que tous les cas, substi l'une à l'autre.

La Cataine (Nepeta cataa ou IIerbe aux chats, Merh de chat, doit son nom. singulière attraction qu excrce sur les chats qui se sent à la manger et à se trer dessus. Elle croit in tous les terrains et à to les expositions. On réc pendant tout l'été les somi tés fleuries dont l'odeurs rapproche de celle de la In the. Les fouilles fraîches, chées excitent la sécrétiord la salive, et bar une sortd 
ivation, peuvent ainsi soulager ou faire dispa. re des maux de dents.

a Lavande (Lavandula spica) s'appelle aussi Laide en épis, Lavande aspic, Spic, Aspic, Faux d. Ses tiges grêles, à rameaux dressés, nomux, atteignent environ 75 centimètres, elles por t de petites feuilles étroites, lancéolées, d'un vert nchâtre. Ses petites fleurs, d'un bleu violacé, lquefois blanches, sont groupées en verticilles iguliers formant un épi terminal. On récolte les umités fleuries en juin-septembre avant l'épaissement complet.

a Lavande doit sa saveur chaude, un peu ère, et son odeur agréable à une huile essenlo riche en camphre, connue dans le commerce s les noms d'huile de spic ou d'aspic

es abeilles sont très-friandes de la plante que si, dont la tige rameuse, haute de 75 centimè;, porte des feuilles ovales, dentées, d'un vert cé et de petites fleurs blanches ou d'un rouge lacé qui se développent de juin à juillet. C'est la Isse (Melissa officinalis) ou Mélisse citronelle, ronade, Pouchirade, Piment des rûches. Ello it spontanément dans les Alpcs, les Pyrénées et si dans les environs de Paris; on la cultive dans jardins. Les feuilles, cueillies avant l'épanouisient des fleurs, exhalent, lorsqu'on les froisse, i) odeur de citron qui se change légèrement en e de punaise lorsque les fleurs sont fanécs. -e Calanent (Melissa calaminta), que l'on rentro dans les pâturages secs, sur les coteaux, ite de 3 à 6 décimètres, à fleurs violettes et pur- 
purines, qui durent de juillet à septembre, poury se substituer à la Mélisse.

On emploie la Mélisse comme stimulant et ar spasmodique dans les affections nerveuses : $p$ pitations, hypochondrie, vertige, migraine. $\mathrm{F}$ forme la base de l'Eau de Mélisse des Carn d'un usage fort répandu, que l'on prépare com suit: On met dans une cruche de grès 3 litres d' prit de vin à 85 degrés centigrades, 500 gramn de sommités de Mélisse, 125 grammes de zeste citron, 15 grammes d'Angélique. Au bout d'v dizaine de jours, on passe en exprimant à trav un linge, et l'on ajoute : coriandre 200 gramm - noix muscade 40 grammes, cannelle 40 grammes quelques clous de girofle; au bout de huit jor on passe et l'on filtre.

Les préparations les plus usitées des Labiées si l'infusion, pour laquelle on emploie de 15 à $40 \mathrm{gra}$ mes de sommités fleuries par litre d'eau, selon 1 nergie de la plante et l'effet à obtenir ; le vin, dé lequel on fait infuser la même quantité, dont administre de 50 à 100 grammes; l'huile essentie à la dose d'une à trois ou quatre gouttes sur morceau de sucre ou dans une potion. Pour bains, on emploie quelques poignées de plante.

Telles sont, mes amis, les notions indispensab que je confie à vos souvenirs, au sujet de la no breuse et bienfaisante famille des Labiées.

Arrêtons-nous sous ce grand arbre, au tro: droit revêtu d'une écorce cendréo. Examinez s feuilles composées à folioles ovales, lancéolér 
ntelées. Ses fleurs n'ont point de pétales; son it est un akène muni d'une membrane oblongue i le fait appeler samarre. En mai ou juin, suiat le climat, les feuilles laissent suinter une tière visqueuse, c'est le temps favorable pour récolter.

Thacun de vous connaît son nom : c'est le FrÊNE axinus excelsior), de la famille des Oléinées, it les feuilles sont recherchées par les moutons, chèvres, les boeufs, les chevaux, et communint une saveur désagréable au lait des vaches

en mangent habituellement. On détache au atemps l'écorce sur des branches de trois à quaans. Celle-ci possède, comme les feuilles, une eur amère, âcre, astringente; elle contient assez tannin pour être employée au tannage. On en pare une décoction (30 à 60 grammes par litre iu) que l'on fait prendre dans l'intervalle des ès des fièvres intermittentes, ou bien on l'admire en poudre à la dose de 10 à 24 grammes réée trois ou quatre fois par jour, pendant pluurs jours, dans l'intermission des accès. Sans - plus infaillible que les autres fébrifuges, c'est remède sur lequel on peut compter.

in emploie avec avantage, dans le traitement de ;outte et du rhumatisme, la décoction de 50 à grammes de feuilles sèches par litre d'eau, prise petites tasses deux ou trois fois par jour après igestion. Ce médicament d'un effet purgatif un inconstant, agit sans doute par dérivation de lammation sur le canal intestinal.

nfin la décoction de semences (10 à 30 grammes litre d'eau) est diurétique, et à forte dose purge ux que les feuilles. 
La manne est un suc qui découle d'incision:la tes, dans les climats méridionaux, au tron quelques variétés de Frêne. La cantharide avide de ses feuilles et l'on récolte ces insecte secouant. le matin les branches oủ ils sont eng dis par la fraîcheur de la nuit. Son bois ron est recherché pour l'ébénisterie; avec les gr branches, on fabrique des instruments aratc des brancards; ses feuilles fournissent aux bes un fourrage d'hiver.

La famille des Asparaginées, qui nous a fourni comme sujets d'étude, outre l'Asperg Petit houx et le. Muguet, nous offre ici une $p$ dont la tige souterraine, accompagnée de ra adventives, peut servir de type de ce que l'or pelle un rlizôme, c'est le ScEau de SaLomon vallaria púlygonatum) ou Muguet anguleux, nouillet, Signet, Herbe aux panaris, commun les bois, les lieux ombragés. La tige aérienne atteint de 30 à 60 centimètres, porte de gra feuilles ovales oblongues; de leur aisselle pen de petites fleurs d'un blanc verdâtre, tubuleus sans calice.

La racine, d'une saveur douceâtre, visqueuse pcu âcre, est légèrement astringente, mais au de vue médical je ne puis que vous dire de ne ajouter foi aux propriétés qu'on lui accorde gratuitement. Dans les campagnes, on vante, hitter la maturation, puis la cicatrisation des ris un cataplasme formé de la racine cuite so cendre et broyée avec quantité égale de saind Comme maturatif, l'oignon et le saindoux sont férables; et pour favoriser la cicatrisation 
7.

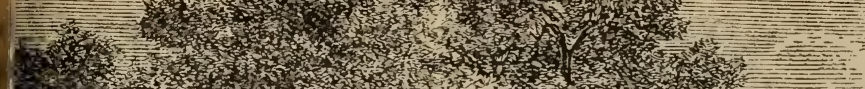

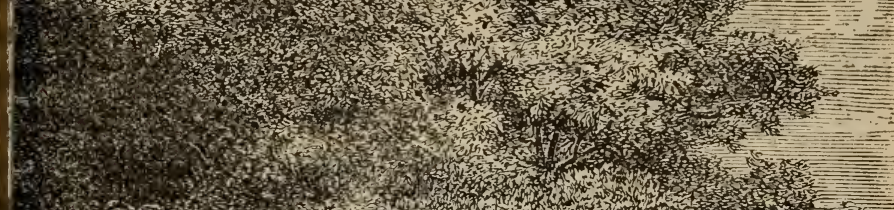

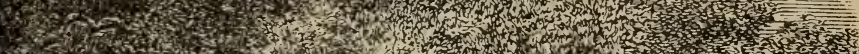

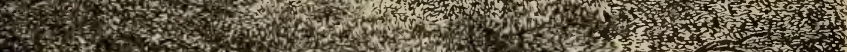

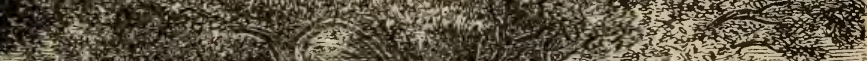

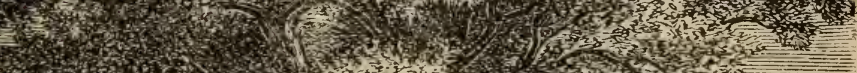

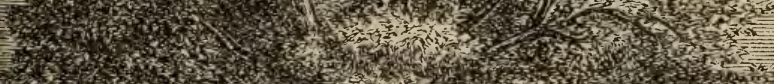

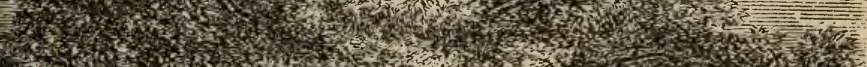

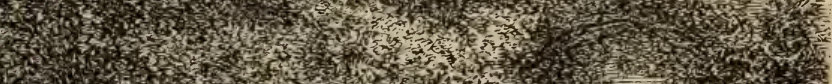

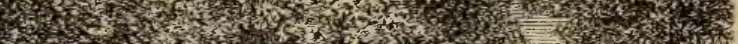

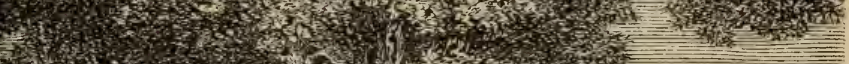

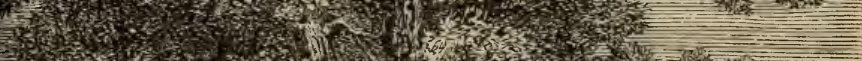

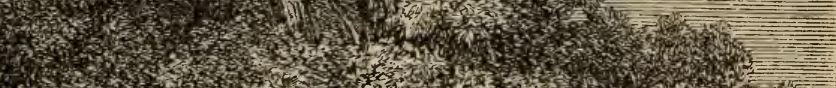

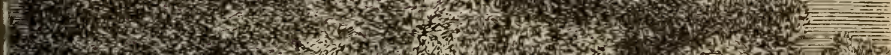

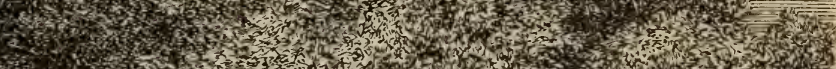
15.

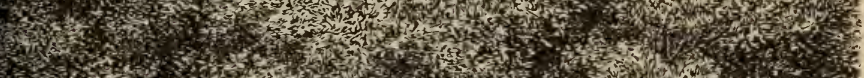

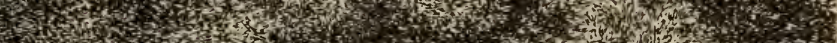

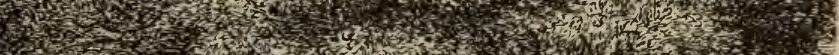

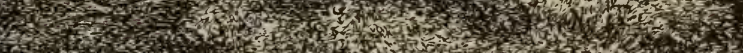

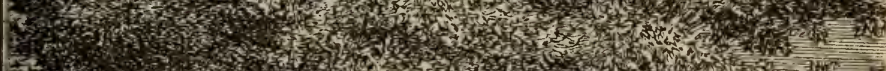

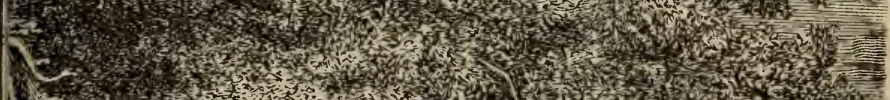

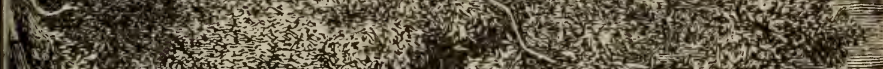
(5)

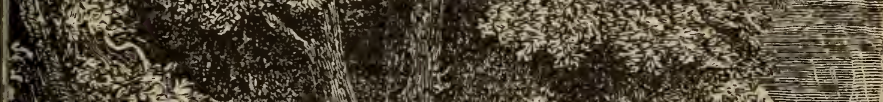
3.9. 25: (5) 
96 LES REMEDES DES GHAMPS

trouve beaucoup mieux de lotions d'eau-de-vie ble. D'ailleurs, toutes les fois qu'un panaris bien reconnu, le moyen le plus prompt et le $p$. sûr de s'en débarrasser consiste à le fendre sul

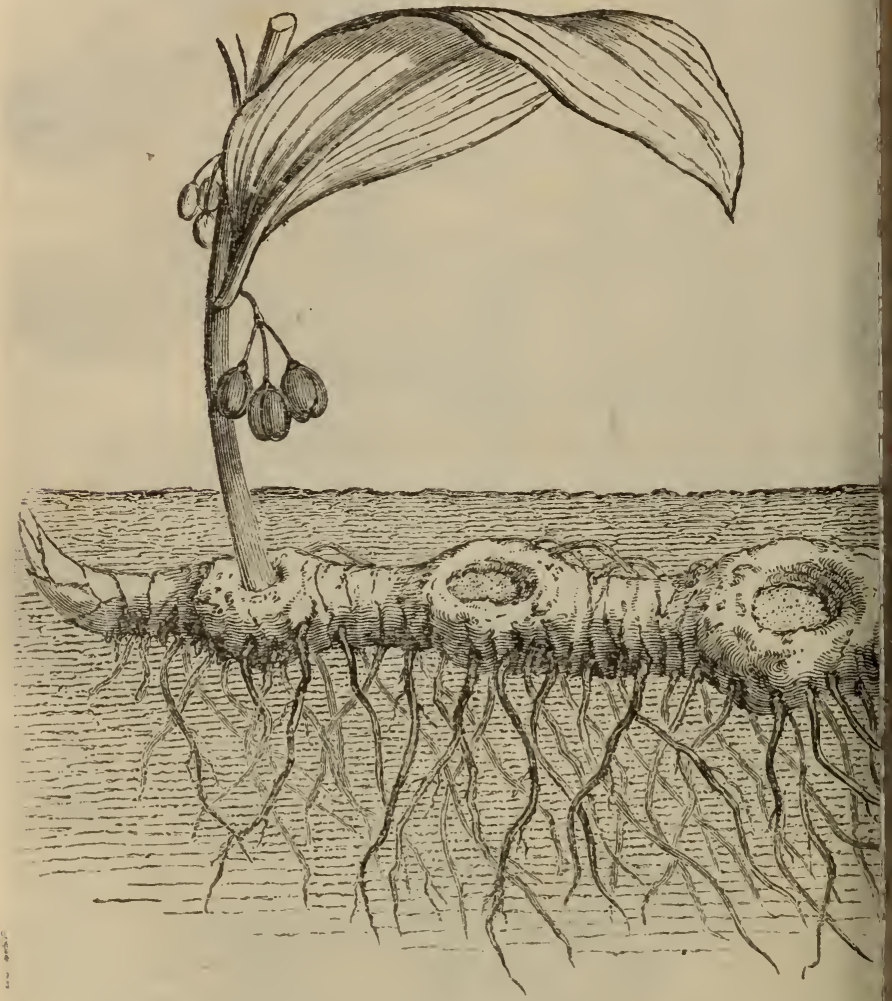

Sceau de Salornon.

samment pour donner, de bonne heure, une isse au sang décomposé; on évite ainsi beaucoup souffrances inutiles, et l'on prévient les accide:s causés par les progrès du mal. 
Vous avez vu souvent dans les bois et les prés umides ou sur le bord des ruisseaux, la ReINE DES és (Spirea ulmaria), de la famille des Rosacées, nnue sous les noms populaires de Spirée, Uliire, Ornière, Barbe de chèvre, Herbe aux abeilles, ed de bouc, Vignette, Grande potentille. D'une ache assez grosse, noirâtre en dehors, sort une e garnie de grandes feuilles composées vertes en isus, d'un blanc cendré et pubescentes en dessous. s fleurs blanches, très-petites et très-nombreuses, ment en juin-juillet, à l'extrémité des rameaux, ; panicules corymbiformes.

Par la dessiccation les feuilles prennent une teinte t-grisâtre, mais les fleurs, jaunies, conservent, n qu'atténué, leur arome primitif. Les feuilles et tout la racine contiennent du tannin, leur saur est légèrement styptique. On retire des somtés fleuries une couleur jaune, solide, employée teinture. Pour l'usage médical, on récolte ces nmités avant l'épanouissement complet - des urs.

e thé des fleurs, plus agréable que celui de Suu, peut le remplacer comme sudorifique; lorson n'a pas besoin d'un agent actif la décoction la plante entière est légèrement astringente et ique.

in somme, la Reine des prés ne mériterait guère sortir de l'oubli dans lequel elle est longtemps neurée, si l'on n'avait constaté que la racine, la 3, les feuilles et les fleurs possèdent des propriédiurétiques incontestables que l'on a mises à fit dans diverses espèces d'hydropisies. On adaistre par verrées l'infusion ou la décoction pré'ée avec 10 à 30 grammes de plante par kilo-

2. PARTIE. 
gramme d'eau. Cette préparation dont l'usage dt être continué assez longtemps, offre l'avantage ne pas fatiguer l'estomac et de ne pas occasionı de troubles nerveux.

Cette plante dont je viens de vous distribuer $1 \mathrm{~s}$ exemplaires est le CHARdon-BÉNIT (Centaurea bel dicta) appelée aussi Centaurée bénite, Cnicus bér: On le cultivait depuis longtemps dans les jardinà cause de ses propriétés médicinales, comme pla importée de l'Inde, lorsqu'on reconnut qu'elle cr spontanément dans nos départements méridiona1 Heureusement elle ne cessa pas d'être réputée $u$ lorsqu'on sut qu'elle ne venait pas nécessairem de loin. La disposition de ses fleurs qui forment capitule terminal composé de vingt à vingt-c fleurons jaunes, auxquels succèdent de petits frı longs à aigrettes, vous la font reconnaître faci ment pour une Composée. La racine est blanc fibreuse, rameuse; la tige rameuse, cannelée, $r$ geâtre, couverte d'un duvet laineux, s'élève à 30 40 centimètres. Les feuilles alternes, poilues, p fondément dentées, portent une petite épine à chaci denture; on récolte en juin, avant l'entier épanors sement des fleurs, les feuilles et les sommités.

Cette plante est douée d'une saveur amère inter mais dont l'effet est peu durable; elle pouri servir à la place de houblon dans la fabrication. la bière. A doses ordinaires elle est sudorifiq diurétique, vermifuge, tonique et fébrifuge. A 1 s térieur, sa décoction modifie heureusement ulcères de mauvaise nature. On peut l'emplo comme succédané de la Centaurée et de la Gentil lorsque les circonstances n'exigent pas une mel- 
cation très-active : elle rend des services incontestables dans la faiblesse d'estomac, la dyspepsie, les fièvres intermittentes.

On administre l'infusion préparée avec 15 à 60 grammes de plante par litre d'eau ou de vin; le suc rougeâtre, qui gonfle les feuilles un peu avant la floraison, à la dose de 30 à 100 grammes; on excite et déterge les ulcères avec la décoction concentrée ou la poudre des sommités.

On peut considérer comme tonique amer du même genre, le Chardon Marie (Carduus Marianus) ou Artichaut sauvage, Chardon argenté, Chardon Notre-Dame, dont les têtes assez coriaces remplacent quelquefois celles d'artichaut, et qui est remarquable par ses grandes feuilles épineuses tachetées et marbrées de blanc.

La Verveine (Verbena officinalis), appelée communément Herbe du foie, Herbe sacrée. Herbe de sang, est le type de la famille des Verbénacées. On la rencontre sur le bord des chemins, des haies, dans les lieux incultes. Les tiges triangulaires, rougeâtres, cannelées, portent des feuilles profondément découpées en lobes inégaux; les petites fleurs d'un blanc violacé, qui s'ouvrent en juinaoût, sont disposées en épis très-allongés. On cueille, avant la floraison, les feuilles et les sommités que l'on fait sécher promptement afin qu'elles ne se décolorent pas.

Autrefois la verveine guérissait les fièvres intermittentes, la pierre, la jaunisse, l'hydropisie, les oliques, les maux de gorge, les vapeurs, les ulcères, la pleurésie, la céphalalgie, l'ophthalmie; ou du noins la crédulité, les préjugés populaires, des 
expériences conduites sans méthode, lui avaien conservé les vertus légendaires qu'o.

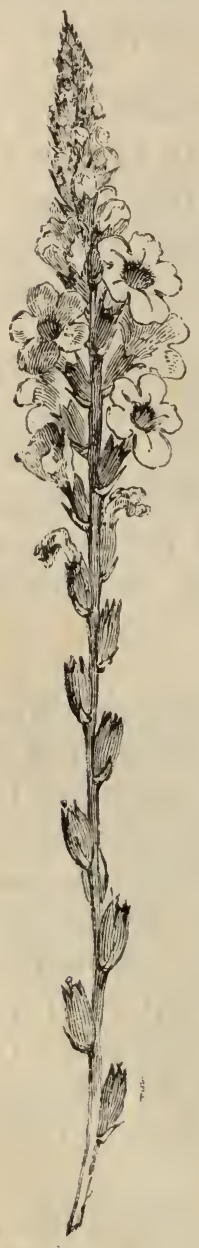

Épi de Verveine. lui attribuait dans les temps an ciens.

Aujourd'hui, il est bien reconn que cette plante sans arome, mais lc gèrement amère, et qui contient un très-faible proportion de tannin, $n$ peut guère rendre de services comm remède interne.

Il est vrai que les feuilles écrasée: cuites dans du vinaigre, et appl quées en cataplasme sur le côté o sur la tête, calment parfois les $\mathrm{m}$ graines ou le point de côté des pleu résies, mais on doit, dans ces cai tenir compte de l'action légèremer révulsive du vinaigre.

La culture a produit de remarqua bles variétés de Verveines blanches roses, rouges et violettes. On pei les semer en place dans les parterres au commencement du printemps elles fleurissent pendant toute la bell saison. On les multiplie aisément $d$ boutures et de marcottes faites à 1 fin de l'été. Si l'on rentre pendar l'hiver, dans la serre froide, ce jeunes plantes mises en pots, on a $d$ bonne heure des sujets vigoureu dont on forme des massifs de cou leurs assorties.

Je n'ai guère besoin de vous décrire le CiIÈvaE 
FEUILLE (Lonicera periclimenum), type do la famille des Caprifoliacées, qui croit spontanément dans les haies et les bois et dont on cultive plusieurs espèces ou variétés. Les feuilles sont souvent perfoliées, c'est-à-dire qu'elles embrassent toute la circonférence de la tige, qui semble les traverser. Les fleurs, disposées en fascicules à l'extrémité des rameaux, présentent une corolle ménopétale en tube très-allongé, fendue en deux lèvres, dont la supérieure est incisée en quatre lobes peu profonds, tandis que l'inférieure, très-étroite, est roulée en dessous; elles produisent des baies d'un jaune clair. A défaut d'autres agents plus actifs, on pourrait employer l'infusion des fleurs sèches (4 à 8 gramnes par litre d'eıu), comme antispasmodique et uussi comme tonique dans les catarrhes, mais il ist rare que l'on n'ait pas sous la main des prépaations plus sûres et plus énergiques.

Je crois néanmoins que le Chèvrefeuille est un rès-bon remède, à la condition de le laisser là oủ est, dans les bois, les buissons, qu'il égaie et u'il embaume. Conduisez les malades, les convaiscents, dans les endroits privilégiés, où il pourra 3 respirer en pleine nature, et vous ne pourrez Lanquer de constater les heureux effets dos influen. es printanières su: les organisations que la malaie a rendues éminemment susceptibles de recevoir de transformer les impressions.

C'est là une médication bien simple, que l’on apprécie pas assez. L'exercice modéré, l'air pur is champs ensoleillés, la vue de paysages, de antes, que des souvenirs rendent chers, le chant oiseaux favoris, les parfums agrestes dont les fluves pénétrantes ravivent toutes les facultés et 
toutes les fonctions; toutes ces influences employées à propos, sont plus utiles que les préparations plus ou moins déplaisantes auxquelles on réserve à tort le nom de remèdes : un rayon, un chant, un parfum peuvent être des remèdes aussi. 


\section{QUATRIËME SÉRIE DE PROMENADES}

(JUILlET, AOUT, SEPTEMBRE.)

Avant que les remèdes exotiques eussent envahi 3 pharmacies et pris la place des produits de nos mpagnes avec lesquels nos aieux savaient fort en se guérir, cette petite plante dont j'ai arraché :elques pieds pour notre leçon était réputée l'une s plus utiles parmi celles qui croissent spontanéont dans nos climats. L'Ipecacuanha ayant été porté d'Amérique, réunissant deux inconvénients - l'esprit humain a transformés en qualités de nvention : venir de loin et coûter cher, l'humble inte de nos bois a été bannie de la médecine urine. Aujourd'hui, c'est à peine si, dans les camgnes, on conserve la tradition des services qu'elle idait autrefois et qu'elle tient toujours aux orzs de ceux qui savent l'employer à propos. C'est .SARET (Asarum europœum), que l'on appelle ssi Asarine d'Europe, Oreille d'homme, Oreilte, Rondelle, Nard sauvage, Cabaret, Roussin, :ard. Il appartient à la famille des Aristolochiées i fournit beaucoup d'espèces précieuses à la mé:ine, mais la plupart exotiques.

ta racine est une souche horizontale ou rhizôme isse comme une plume d'oie d'un brun grisâtre, leuse, munie de fibres blanchâtres. Les tiges sque nulles se terminent par deux feuilles en me de rein, coriaces, vertes et luisantes en dessus, 
pâles et duveteuses en aessous, portées par un lo; pétiole. Les petites fleurs solitaires, d'un pour noirâtre, se montrent en avril et mai à la bifur tion des pétioles, ce sont des clochettes, formées $\mathrm{p}$ le calice. On récolte les feuillos pendant l'été ; le: odeur, leur saveur et leurs propriétés sont, à degré un peu moindre, celles do la racine. Celle doit se récolter deux fois par an: au printens avant la floraison, et à l'automno, car ses proprié changent rapidement à mesure qu'on la conser Fraîche elle est vomitive et purgative; après mois elle n'est plus vomitive; après deux ans e ne purge presque plus, mais elle possède alors qualités diurétiques. Fraîche ou récemment sécl elle est douée d'une odeur camphrée et térébenthir agréable et pénćtrante; sa saveur, comme celle feuilles, est âcre, amère, nauséeuse.

Toute la plante est excitante, émétique, purgati anthclnintique et sternutatoire. Lorsque l'on propose seulement d'opérer une perturbation al rante dans la bronchite chronique, la diarrhée coqueluche, de 10 à 20 centigrammes de poudre racine remplissent très-bien le but. Pour fo vomir, on donne de 60 à 80 centigrammes; 1 dose plus forte agit en même temps sur l'eston et l'intestin. Si l'on commence par une ou de doses d'Asaret le traitement des fièvres quartes, en vient facilement à bout. Les vétérinaires l'e ploient contre le farcin et les vers. Le nom de baret lui vient de ce que les ivrognes en ont pour se faire vomir afin do recommencer à boi mais c'est en même temps un remède contre la $r$ ladie qui consiste dans le désir irrésistible de : ritueux; il relève l'appétit et, pour peu que le ti 
ment moral lui aide, dissipe ou éloigne le besoin ictice.

La poudre des feuilles, qui peut dans tous les cas mplacer, - mais à dose un peu plus forte, - celle e la racine, est un bon sternutatoire, utile dans s maux de tête invétérés; fraîches, ces feuilles nt sialagogues, c'est-à-dire augmentent, lorsqu'on s mâche, la sécrétion de la salive, et peuvent ainsi lmer quelques névralgies des dents.

Toutes les autres plantes dont nous allons nous ccuper aujourd'hui appartiennent à la famille des mbelliféres, qui nous a dẻjà fourni une espèce nportante, l'Angélique. Toutes offrent, à des derés divers, un caractère dominant, celui d'agir sur organisme comme stimulant des actions vitales. lais en outre de cette propriété commune, chaque pèce est douée de vertus spéciales que nous allons udier en détail.

L'Ache (Apium graveolens) ou Céleri des marais, éleri sauvage, Persil odorant, est une plante bisinuelle de 60 à 90 centimètres de hauteur, qui oît communément dans les lieux humides. Culvée depuis longtemps dans les jardins elle a prolit une variété qui est le Céleri ou Ache douce, ais une sous-variété nommée Céleri-rave à cause 3 la forme globuleuse de sa racine charnue. Les hevaux ne touchent jamais à ses feuilles une ou uux fois décomposées, solides, larges, incisées ou ontelées, mais elles sont mangées par les moutons, s chèvres et les vaches. Les tiges ressemblent ;sez à celle de l'Angélique; les fleurs très-petites, cinq pétales disposés en roue, forment des om’lles à l'aisselle des feuilles et à l'extrémité des 
rameaux. On ernploie fraîches les feuilles d'un odeur aromatique particulière et d'une saveur âcre La racine qui est bisannuelle, courte, pivotante, ra meuse, roussâtre en dehors, blanchâtre en dedans doit être récoltée pendant la seconde année; c'es alors seulement qu'elle possède toutes ses pro priétés actives. La dessiccation lui fait perdre so odeur désagréable, un peu vireuse. Quant au frui qui est un akène oblong, rayé, grisâtre, il est aro matique, stimulant, stomachique.

La décoction des feuilles (30 à 60 grammes pa litre d'eau) coupée avec du lait frais et prise à jeur a été utile dans l'extinction de voix, l'asthme hu mide et le catarrhe pulmonaire chronique. Le feuilles pilées et appliquées, seules ou avec additio de vinaigre et de sel, sur les contusions, les engo: gements froids, les engorgements laiteux, agisser comme résolutif. Leur suc est antiscorbutique, c'es un bon gargarisme pour les ulcérations de la gorg un topique détersif pour les ulcères cancéreux.

Enfin, pour guérir les fièvres paludéennes intel mittentes avec leurs complications, engorgemer de la rate, gonflement des pieds, etc. On obtier d'excellents résultats en faisant prendre en tro doses, entre les accès, de 150 à 200 grammes de si de feuilles d'Ache dans une décoction concentrée c la racine sèche. Il faut noter que ce traitemen comme toutes les médications fébrifuges, doit êt continué pendant deux ou trois septennaires apr la cure apparente, en ayant soin toutefois do dim nuer et d'éloigner les doses; que l'on abrége assure la guérison par une bonne nourriture, air sain, et l'usagge de toniques amers et astril gents. 
spécimen quo je tiens ici vous est certainensent lier, car cette plante, qui croît spontanément 1) lo midi de la France, est cultivée dans tous ardins potagers pour les besoins de la cuisine; un assaisonnement agréable, toujours prêt, et ne coûte rien.

3 Persil (Apium petroselinum), appelé dans ques campagnes Persin ou Ache persil, fréquemt employé par les paysans dans leur médetraditionnelle, a été étudié à nouveau depuis que temps par les médecins et les chimistes. science officielle y ayant reconnu la présence principe actif bien déterminé, de nouvelles riences ont confirmé les données existantes sur bropriétés thérapeutiques. Parmi les végétaux s que nous avons étudiés, un grand nombre dent encore cette sanction ou ce passe-port de ience doctrinaire, et guérissent tout bonnement jonnes gens qui y croient parce qu'ils les ont à l'œuvre.

n'aurais pas besoin de décrire une plante si lière, s'il n'était fortimportant de savoir la difncier de la Petite ciguë. Celle-ci exhale une ir nauséeuse, la racine est petite, la base de la est violette ou rougeâtre, couverte d'une pousglauque; ses feuilles, plus aiguës que celles jersil, sont d'un vert noirâtre. La racine du Perst assez grosse, les tiges atteignent près d'un : de hauteur, elles portent des feuilles compod'un beau vert, les fruits sont ovoïles, allonC'est une plante bisannuello qui ne monte à ne que la seconde année.

3 Persil vient dans tous les terrains, mais il ère les terres fraîches et légères peu fumées. En 
hiver il faut le couvrir de paillassons pour le pré r

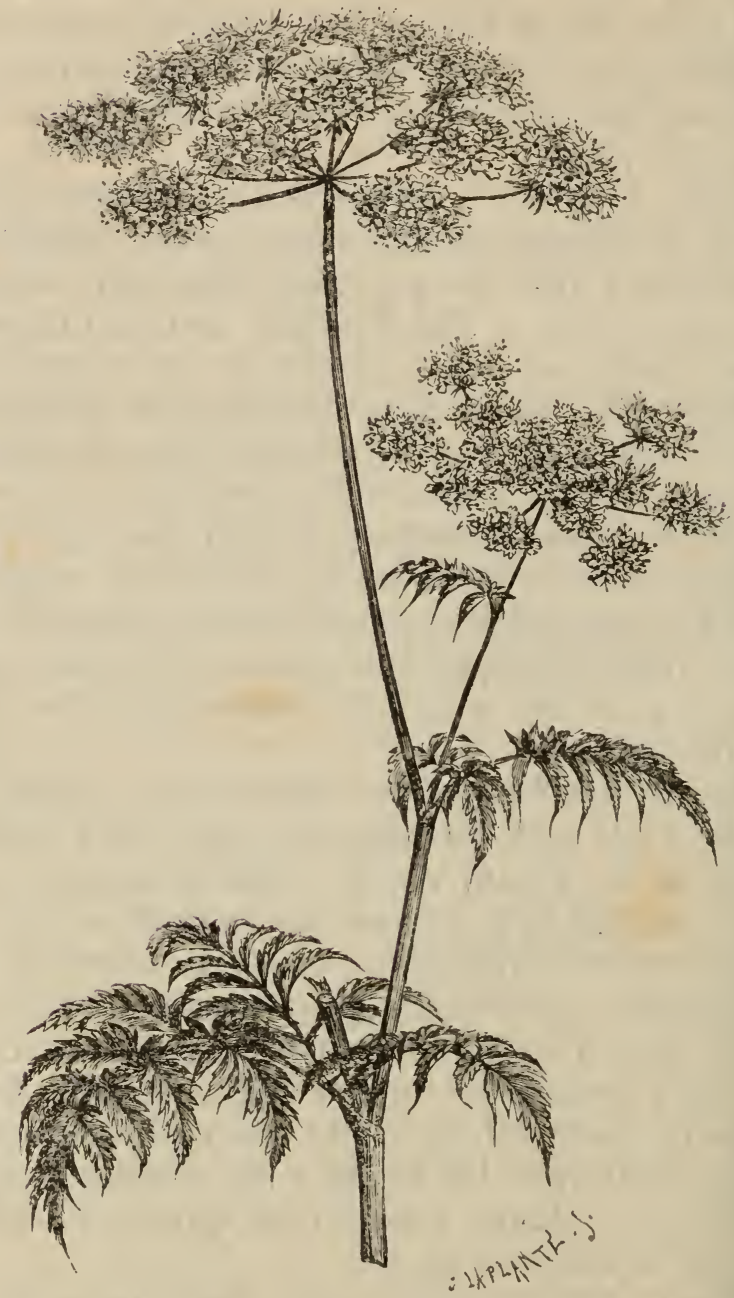

Sommité de Persil.

ver de la gelée à laquelle d'ailleurs il résiste mics 
tre un mur exposé au nord qu'à l'exposition du li. On le sème depuis le printemps jusqu'à la de l'automne. Si l'on coupe les tiges à mesure elles se montrent, on peut le faire durer trois On cultive deux variétés de Persil frisé dont très-petite qui se reproduit bien de graine, disque l'autre dégénère quelquefois en l'espèce imune

a décoction de racines de Persil, fraîches ou zes à la dose de 30 à 90 grammes par kilomme d'eau, est stimulante et apéritive, c'est-àcapable d'ouvrir une voie d'élimination par les ies ou les sueurs dont elle provoque la sécré; aussi rend-elle des services dans les engorients du foie, l'hydropisie, les irréğularités de irculation du sang. Les feuilles pilées applias sur les contusions, les tumeurs froides, les orgements laiteux produisent le même effet rétif que les feuilles d'Ache, en stimulant la cirtion et l'absorption dans la partie affectée; elles aussi la propriété d'aviver, de déterger les ul; de mauvaise nature et de les pousser à la risation. Ce topique peut rendre des services les ophthalmies purulentes. Pour ce qui est de mmploi pour hâter la cicatrisation des coupuil est fondé sur un préjugé populaire qui a fait hter une foule de recettes destinées à ce but. Mais fua les bords d'une coupure ou d'une plaie simont rapprochés avec soin et maintenus en place, ture se charge de la guérison soit en produisant réunion immédiate, véritable soudure des tisqui commence au bout de quelques heures et ure qu'une semaine ou deux ; soit par la proon de tissu intermédiaire qui remplit les espa- 
ces vides et réunit les parties lésées. Dans le mier cas, il n'y a absolument rien à faire, or peine de contrarier le travail naturel de réparajr dans le second, comme il y a formation de $u$ l'eau tièdo est le meilleur cicatrisant. Cepera: chez les sujets d'une mauvaise constitution ou mis à une hygiène défavorable, il arrive que $u$ fois que les plaies, les simples coupures, au li tendre naturellement à la cicatrisation, s'ulcer t. se recouvrent d'une espèce de membrane d'oil' coule un pus couleur de rouille, d'une odeur reju sante. C'est alors que l'on emploie avec profil cool dilué ou les remèdes que nous avons ap détersifs pour faire tomber la membrane, ar les bourgeons charnus, faire sortir un pus bn et ramener l'ulcère à la condition de plaie ordiri La propreté locale et générale, un air salubrefi. quemment renouvelé, une bonne nourriture 10 d'ailleurs indispensables au succès. De plus, tat anormal de la plaie est causé ou mainten un vice du sang, par une maladie chroniqu doit combattre ces causes dans leur source $p$ remèdes appropries. Les stimulants et les ton amers seront presque toujours utiles.

Mais revenons au Persil pour constater qu'ip sède comme l'Ache des propriétés fébrifuges ito testables. Il convient de donner de 150 à 200 g mes de suc au moment de l'accès, lorsqu'il seulement de combattre les fièvres de printen. d'automne de nos climats.

Les détails que je vous ai donnés sur le Pers dispensent de vous parler longuement du CEret (Scandix cerefolium) dont les propriétés, urp 
noins actives, sont de même nature, mais sur lequel on ne peut guère compter comme fébrifuge. En evanche, lo suc de celui-ci est plus efficace commo pplication externe dans les ophthalmies. Notez outefois que si la maladie des yeux est liée à une raladie constitutionnelle, scrofuleuse ou autre, auun remède local ne rocurera une guérion durable si l'on ne étruit en même mps, par un traiteient interne, la cause es accidents locaux. y a une variété de erfeuil dite CERFEUIL USQUÉ ,

(Scandix dorata) doué de proriétés plus énergiues; les racines charues et féculentes du ERFEUIL BULBEUX Yhorophyllumbulpsum) sont alimenires; mais il faut iter le Cerfeuil sau-

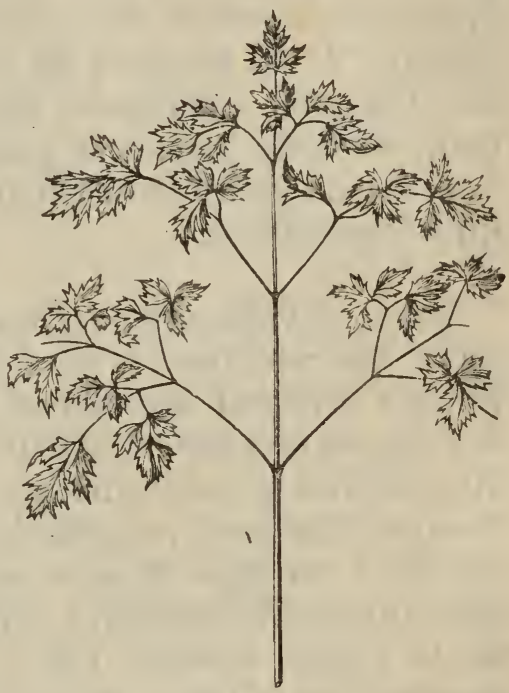

Cerfeuil.

IGE (Chœrophyllum sylvestre) ou Persil d'âne, ai se trouve dans les endroits humides et courrts, car il agit à la manière des poisons narcoti1-âcres. Ses tiges n'atteignent pas plus de 50 à 60 ntimètres, les folioles sont un peu pliées en goutzre, il ressemble assez à la Ciguë.

Le Phellandre (Phellandrium aquaticum) ou guë aquatique. Fenouil d'eau, Millefeuilles, aqua- 
tique, Persil des fous, a beaucoup de rapports a :o la Ciguë, mais il habite toujours l'eau ou les lir $\mathrm{x}$ marécageux ; de plus, il exhale une odeur aromatiq contient un suc incolore, et ses tiges ne sont tachées de pourpre. Mangé vert par les chevauxil les paralyse. Il exerce une action notable sur le $\$ 3$ tème nerveux, et à faibles doses, agit comme cmant sans donner lieu aux effets désagréables l'opium. Il est prudent de ne l'employer que dis les affections catarrhales chroniques. On donne deux en deux heures la poudre des semences dose $1 / 4$ de gramme augmentée graduellement j3. qu'à 2 grammes.

Quant à l'Enanthe (Enanthe cricata) ou P sacre, qui croit dans le nord et l'ouest de la Fran je vous inviterai seulement à la reconnaître por n'y jamais toucher. C'est un poison narcotico-âe violent dont la médecine n'a pas encore méthod l'usage. Son port rappelle le Phellandre et le $\mathrm{P}$ sil, mais les tiges brisées ou incisées exsudent suc jaune caractéristique, plus abondant que ce de la Ciguë vireuse. On a proposé cette pla pour guérir la lèpre, mais elle n'a pas été soumis: des cxpériences concluantes et son usage a causé graves accidents. En même temps que nous app nons à bien connaître nos amis, rangeons au mos parmi les suspects les végétaux actifs, vénénei dont les experts seuls pourront découvrir l'utilité la rendre pratique. Surtout gardez-vous bien de vo laisser aller à la tentation d'expérimenter les eff des végétaux qui vous sont signalés comme act et dangereux et de substituer ainsi, au désir bien, la satisfaction d'une curiosité coupable pu 
u'elle vous exposerait à causer de terribles accients. Vous devez vous contenter de suivre les chelins battus, et laisser à des personnes autorisées 3 soin d'élargir le champ de vos travaux.

Si notre herborisation d'aujourd'hui n'a pas été bondante, elle va du moins nous fournir comme jjet d'études des plantes douées de propriétés nergiques et bien définies, fort intéressantes au oint de vue spécial de nos excursions.

Commençons par ce type de la famille des Conolvulacées, le Liseron (Convolvulus sepium), auement dit Liseron des haies, Grand Liseron, isette, Manchette de la Vierge. Cette famille comand des herbes, des arbrisseaux et même des bres, mais la plupart des espèces qui la compoint sont herbacées, à tige longue, flexibto, qui enroule autour des tiges et des branches à sa prtée, d'où le nom de tiges volubiles et aussi le om de la famille. Les feuilles alternes munies un assez long pétiole sont entières ou lobées; les uurs, ordinairement grandes, sont solitaires ou réues au nombre de deux à quatre à l'aisselle de feuil$\mathrm{s}$; la corolle monopétale présente la forme d'une oche ou d'un entonnoir ; avant l'épanouissement le est plissée comme un filtre de papier. Aux fleurs iccède une capsule à deux ou quatre loges, reuverte par le calice. La racine charnue contient 1e résine âcre, purgative, que l'on ne trouve pas, utefois, dans celle de la Patate et de quelques ttres espèces comestibles.

Cette définition généralo me dispense de vous 
décrire en détail la plante si familière qui nous

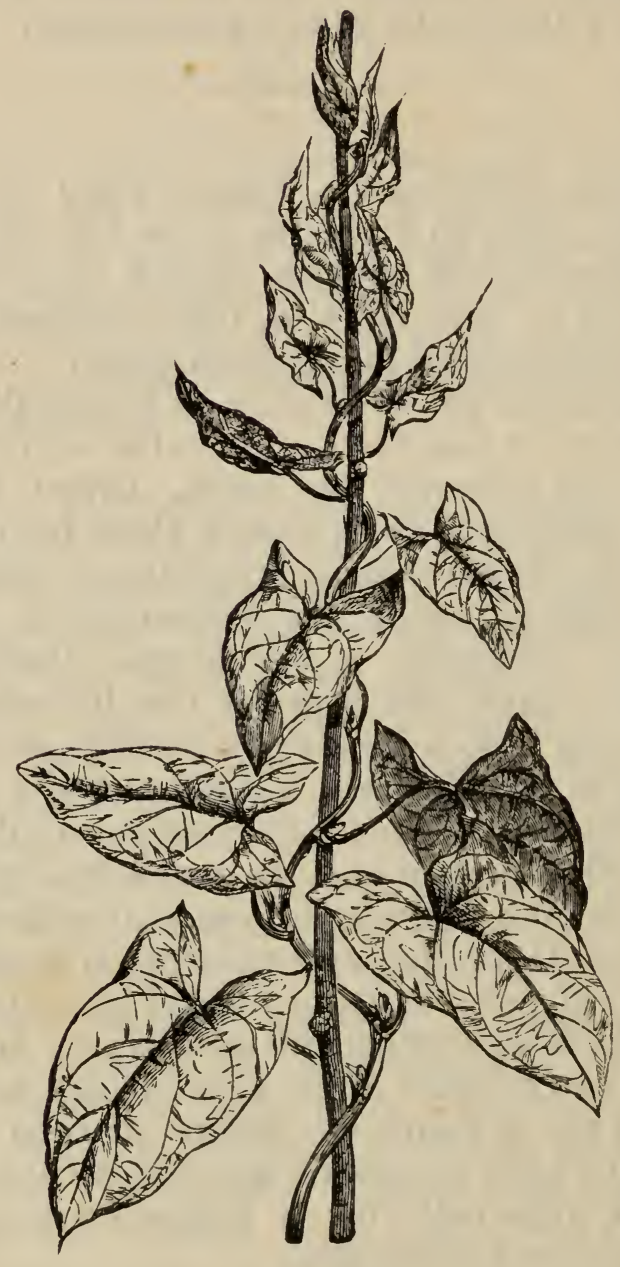

Tige de Liseron.

cupe, remarquable par des tiges longues de $I^{t}$ 
sieurs mètres et ses très-grandes fleurs blanches zans parfum. Le Liseron est très-commun dans les aies, les buissons où il fleurit depuis juin jusqu'en ctubre. C'est aux mois de juillet et d'août qu'i? onvient de le récolter pour en conserver le suc ou a racine, Les feuilles et surtout les fleurs sont mères; la racine possède une saveur un peu âcre. Une dose de 6 à 2 grammes de euilles contuses, nfusées dans de eau, forme une onne potion purative. Pour éviter ute irritation, il st bon d'y ajouter n peu de miel ou iieux du mucilago - racine de Guilauve ou de graine 3 Lin. Sćchées à ombre, pulvéries et mêlées à du iel, les feuilles

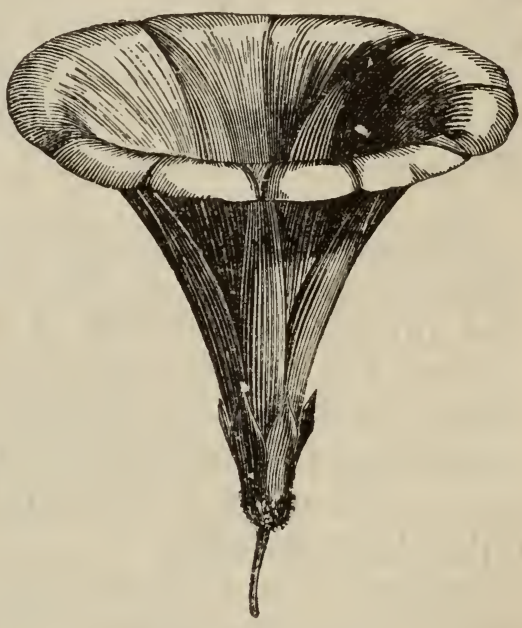

Fleur de Liseron. nservent bien leurs propriétés purgatives. Le suc laiteux de la racine, épaissi en consistance ; sirop, est un des purgatifs les plus efficaces, il ière comme le Jalap, et convient particulièrement la dose de 1 gramme et plus, assez souvent répée dans les hydropisies et les maladies constituinnelles chroniques. Les enfants prennent sans pugnance une dose proportionnée à leur âge. Le Petir Lisenon (Convolvulus arvensis) ou seron des champs, Petit Liset, Campanette, ¿े 
fleurs petites, d'un blanc rosé, est doué de proprié tés analogues à celles du Grand Liseron.

La culture a produit un grand nombre de sous variétés de Liserons de diverses couleurs, que l'c sème en place au printemps et dont les fleurs : succèdent sans interruption depuis l'été jusqu'à fin de l'automne. Ses guirlandes, mariées à cell de la Capucine et du Haricot d'Espagne, forme: aux croisées le plus gracieux encadrement. C plantes amies fournissent à la plus humble ma sarde un jardin de fenêtre et une tonnelle qui exe cent une heureuse influence sur l'esprit et par su: sur la santé de ses habitants.

L'Eupatorre (Eupatorium cannabinum) est u plante vivace, élégante, très-commune dans les pr ries humides, les marais, et au bord des eaux tre quilles. On l'a nommé aussi Origan des mara Herbe de Sainte-Cunégonde. Elle appartient à famille des Composées, qui nous a déjà fourni l'A rone, le Pissenlit, le Tussilage, l'Armoise, la Can mille, la Matricaire.

La tige un peu rameuse, parfois rougeâtre, du teuse, pleine de moëlle, s'élève à un peu plus d mètre. Elle porte des feuilles divisées en trois $s$ ments lancéolés et dentés. Les fleurs, de coul pourprée, disposées en corymbes à l'extrémité rameaux, s'ouvrent de juillet en septembre. On ci cueillir la plante un peu avant la floraison; quar la racine oblique, blanchâtre, un peu fibreuse, la grosseur du petit doigt, d'une saveur amèr piquante mais sans odeur, il faut la récolter printemps. Une partie des contradictions que observe dans l'appréciation des simples provient 
ce que les diverses parties n'ont pas été récoltées, préparées, conservées et administrées ainsi qu'il convenait. Si l'on ne se procure une racine qu'après la maturation des graines, alors que la plante a dépensé à leur profit toute la séve et les principes actifs de sa base, il est certain que l'on n'aura plus qu'une substance à peu près inerte, au lieu d'un médicament énergique.

En médecine pratique, il est bon de se borner à atiliser les propriétés toniques des feuilles d'Eupaoire dont on prépare une infusion à la dose de 30 à 30 grammes de plante par litre d'eau, et la vertu jurgative de la racine qui produit des évacuations ians causer d'affaiblissement. Pour cet effet on idministre la décoction ou l'infusion de 30 à $60 \mathrm{gr}$. le racine broyée dans 250 grammes d'eau ou de vin.

La famille des Euphorbiacées nous a déjà fourni leux espèces intéressantes : le Ricin et la Mercuiale. Nous allons maintenant nous occuper de l'Euhorbe Epurge (Euphorbia lathyris) ou Grande isule, Euphorbe catapuce, Euphorbe lathyrienne, ithymale épurge. Elle est commune sur la lisière les routes, dans les terrains sablonneux et boisés. )'une racine droite, en forme de fuseau, s'élève à mètre 50 centimètres environ une tige lisse d'un ert rougeâtre ramifiée en forme d'ombelle, portant es feuilles lisses, très-étroites, d'un vert bleuâtre. in juin et juillet se montrent les petites fleurs d'un aune verdâtre, renfermées dans des appendices bractées) situés à la bifurcation des rameaux. Les leurs mâles, au nombre de quinze ou vingt, consisent en une simple étamine; elles entourent une leur femelle formée d'un ovaire à trois loges 
muni de trois styles, qui prodnit trois capsule réunies dont chacune renferme une graine dont or extrait par expression une huile purgative. Cett

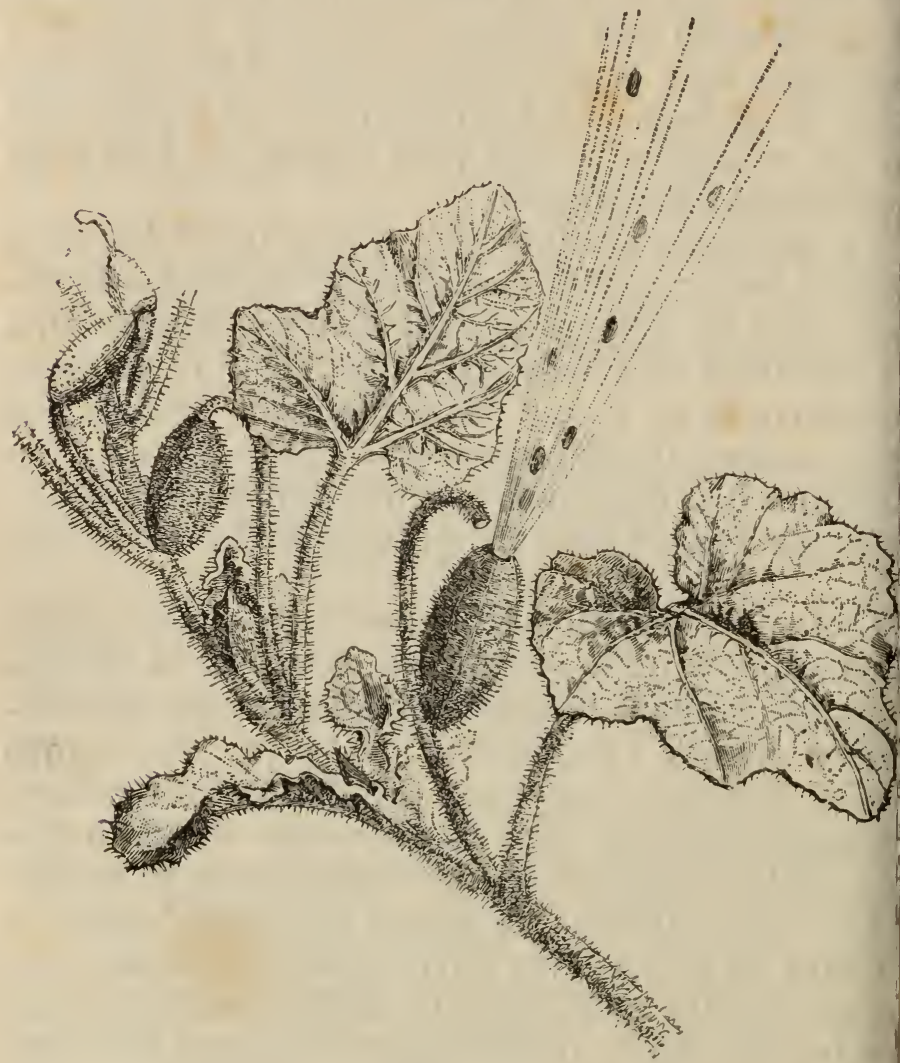

Mornordique.

huile, lorsqu'elle est fraîche, agit d'une maniè très-douce, à la dose de deux à trois gouttes che les enfants, de six à huit chez les adultes; mais ur 
is rance, elle cause des coliques. Appliquée sur la zau elle détermine la formation de boutons, d'amoules, et son action dérivative, qu'il faut d'ailleurs urveiller, peut être utile dans les catarrhes, les svralgies et autres maladies chroniques. L'huile olive ou d'œillette dans laquelle on a fait macérer es graines concassées d'Épurge devient également ritante.

Les paysans emploient souvent pour se purger six à douze graines d'Epurge. Ils les concassent I les mâchent avec soin, selon l'intensité d'effet i'ils recherchent. Quatre ou cinq feuilles, broyées ns du miel, peuvent remplacer les graines. Comme tous les drastiques, l'Epurge a été vanpour la guérison d'une foule de nıaladies, mais ist dans les diverses espèces d'hydropisie qu'elle mble le mieux réussir. Le suc des tiges est vulirement employé pour détruire les cors et les rrues.

De nombreuses espèces de cette plante croissent ns nos campagnes : elles sont moins bien cones, et il est prudent de ne pas les employer. La is active est l'Euphorbe des marais (Euphorbia lustris) nommée aussi Grande Esule, Tithymale is marais.

Vous vous rappelez, mes amis, que la famille des curbitacées nous a déjà procuré deux sujets d'éle : la Citrouillo et la Bryone. Nous allons contier l'examen des remèdes purgatifs drastiques - un autre membre de cette famille. Plus d'un 'mi vous s'est amusé avec les fruits singuliers cette plante sans so douter de ses qualités énerues. 
La Momordique ou Comcombre sauvage (Momo dica elaterium) a reçu les noms de Gôlante, Com combre d'âne. Pomme de merveille. Elle est vivace croit spontanément dans le midi de la France; on sème dans les jardins, au printemps, dans un te rain pierreux et sec.

La racine blanche, charnue, épaisse, assez sen blable à celle de la Bryone, mais moins gross purge sans coliques après avoir été desséchée. Ma c'est le suc des fruits privés de semences que l'c emploie d'ordinaire. Les tiges sont rampantes, sa vrilles, et couvertes de poils courts ainsi que 1 feuilles cordiformes. Le fruit, en forme d'oliv gros comme une petite noix, se détache très-facil ment de son pédoncule, et l'élasticité des parois q se resserrent fait jaillir avec force les graines e tourées d'une espèce de mucilage.

L'extrait du suc de ces fruits, nommé élatériù d'une saveur amère, désagréable, purge ordinair ment à la dose de 30 à 90 centigrammes. C'est purgatif drastique qui exerce une action irritantes l'appareil digestif, et un hydragogue, c'est-à-dire purgatif qui agit spécialement en rendant beauco plus abondante et plus liquide la secrétion des testins, de manière à procurer des selles fluic très-copieuses. Le fluide ainsi évacué étant pris la partie aqueuse du sang, celui-ci a besoin de réc pérer le liquide perdu; si on ne le lui rend $p$ par des boissons et qu'il y ait dans l'organisi quelque dépôt anormal de liquide, comme da l'hydropisie, l'absorption de ce liquide est favoris par l'énergie avec laquelle le sang tend à absork les fluides sur son parcours pour compenser perte qu'il a subie. 
Si on laisse déposer lentement le suc frais de Moordique, on peut recueillir au fond du vase une jèce de fécule cinq ou six fois plus énergique que strait, et qui réclame la plus grande circonspecn dans son emploi.

Quelle que soit la préparation dont on fait usage, est prudent de commencer par des doses trop bles afin d'essayer la sensibilité du malade. On augmente graduellement et l'on continue à proire une purgation copieuse tant qu'il ne survient 3 du côté de l'estomac ou des intestins une irritan qui indique la nécessité de changer de remède de suspendre le traitement. Les émollients, peu ués, sont souvent nécessaires pour calmer l'irriion que l'on a ainsi causée dans l'appareil distif.

Ye que je vous ai dit aujourd'hui et dans d'autres retiens sur les purgatifs et l'indication de leur ploi me permet d'être bref en vous signalant ce-ci. C'est la Coloquinte (Cucumis colocynthis) Jelée vulgairement Chicotin, plante originaire de irient mais naturalisée chez nous où elle vient n de semis dans une terre substantielle, exposée midi.

Les tiges rampantes, anguleuses, poilues, munies vrilles, portent de grandes feuilles à cinq lobes, inchâtres et parsemées de poils à leur face inféure. Aux fleurs femelles, petites et jaunâtres, cedent des fruits d'abord verts, puis jaunes ou aachés, qui atteignent la grosseur d'une orange. masse blanche, spongieuse qu'ils renferment est uée d'une saveur nauséeuse et extrêmement ère. Une dose do 10 à 60 centigrammes de cette 
pulpe tenace pulvérisée au contact de gomme ou sucre bien sec, ou encore 8 à 30 grammes d'un vi dans lequel on a mis à infuser 1 partie de pul

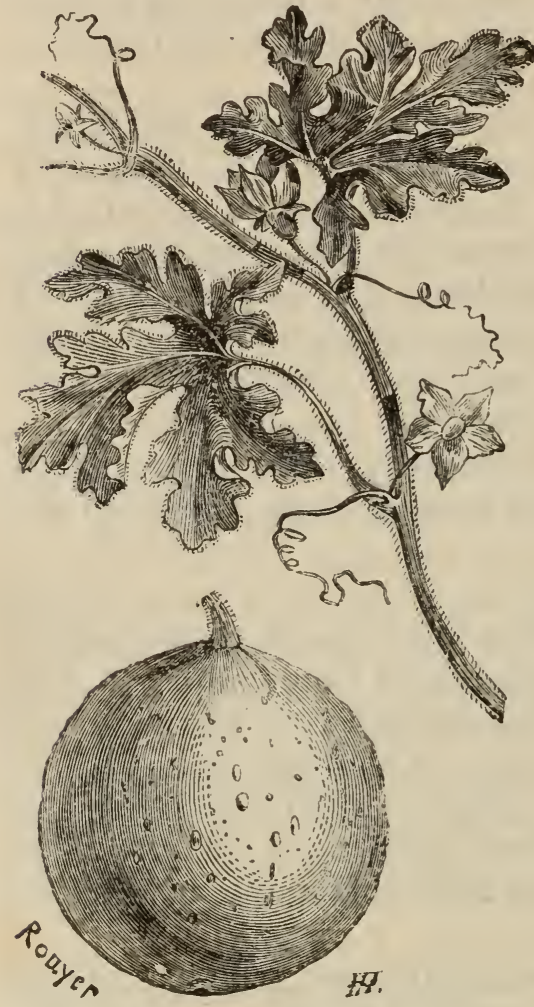

Coloquiate.

sur 6 de liquic constitue un pu. gatif énergique. ( l'emploie avec vantage dans maladies nerve ses, les affectio du foie, les déra gements de la $\mathbf{c}$ culation, la gout le rhumatisn chronique, les e gorgements ato ques, les maladi do la peau.

La pulpe fraîc de Coloquinte, teinture, l'infusi aqueuse, l'extrit appliqués sur ventre, produise au bout de quelq temps le mème eff que si on les ave administrés à l'i térieur, et l'on pe ainsi mettre à profit les qualités vermifuges cette plante, chez les enfants auxquels il est di ficile de faire prendro les préparations d'un go c’ésagréable. 
'our en finir avec le sujet qui nous occupe spélement aujourd'hui je vais vous dire quelques ts de la Gratiole (Gratiola officinalis), de la ille des scrofulariées, c'est une plante vivace l'on troure dans les lieux humides et marécux. On la récolte avant ou pendant la floraiqui a lieu de juin à septembre. La dessiccation fait perdre un peu de son énergie, ce qui n'est un mal. Vous la reconnaîtrez à sa tige herbanoueuse, creusée de sillons latéraux, haute de 45 centimètres, portant des feuilles d'un vert nâtre, ovales-lancéolées dentẻes, à trois nervuet à ses petites fleurs solitaires, tubuleuses, n blanc jaunâtre ou rose. Elle a reçu, selon les tons, des noms très-variés : Grâce de Dieu, ite Digitale, Herbe à pauvre homme, Centaule, Séné des prés, Herbe à fièvre. Voilà certes noms qui valent des titres de noblesse. Je $\mathrm{x}$ bien convenir que la Gratiole les mérite tous, la racine, d'un effet peu constant, est émée à la dose de 1 à 2 grammes, et le reste de la ite agit comme purgatif violent. " Tout est dans ", peut-on dire à propos de bien des remèdes atés " héroïques ", mais à la condition de savoir récier les circonstances favorables à leur usage es principes qui doivent en régler l'administra-

n donne par cuillerées la décoction ou l'infusion à 12 grammes pour 120 grammes d'eau ou de $1 / 2$ à 2 grammes de poudre en pilules ou dans laune d'œuf.

- médecine urbaine a délaissé la Gratiole comme d'autres produits naturels de nos campagnes, ( adopter des végétaux exotiques dont l'emploi 
ne demande pas moins de prudence et de pré tions. Puisse la mode qui envahit même la scico par un de ces retours qui lui sont fréquents aill ramener les esprits observateurs et curieux, a des moyens d'investigation des méthodes model vers l'étude si attrayante de nos remèdes. La réc la culture et le commerce dessimples, leurs pr rations pharmaceutiques, deviendraient pour campagnes comme pour les villes une nou source de richesse. En attendant, lorsque avons à soigner, aidés de connaissances plus moins limitées, l'habitant de la plus modeste c mière, sachons trouver dans le champ, sur la au bord du chemin ou du ruisseau les remèdes la Providence semble avoir semés autour de l'hol partout où la maladie peut atteindre son co commo elle lui donne partout contre les défailla de l'âme des parfums, des couleurs attrayantes chants d'oiseaux et des rayons de soleil.

Asseyons-nous, mes amis, sous ce grand $\mathbf{N}$ qui va nous fournir son ombre, une atmosp balsamique et un sujet d'étude fort intéressan me dis souvent en parcourant nos campagnes : I sommes trop riches, nous sommes gâtés; nous vons guère le temps et l'occasion d'apprécier nos trésors et nous passons avec indifférence de des végétaux qui seraient presque l'objet d'un c dans des régions moins favorisées.

Le Noyer (Juglans regia) appartient à la fanl des Juglandées; il vous est assez familier pour je n'aie pas besoin de vous le décrire. Toutese 
ties de l'arbre, excepté le bois très-recherché ir l'ébénisterie, sont utiles en médecine : écorce tiges et des racines, feuilles, fleurs mâles disees en longs chatons cylindriques, drupe verte u), épiderme de la noix, enfin la noix ellene. L'amande de la noix contient la moitié de poids d'une huile siccative utilisée dans les ;, et que l'on a employée comme toenifuge à la e de 150 grammes à jeun pendant quinze jours. itefois pour assurer son action il faut y faire iner cinq ou six gousses d'ail. L'épiderme qui enppe l'amande, d'une saveur astringente et amère squ'il est frais, est un tonique astringent riche tannin'; il en est de même du zeste qui sépare les es de l'amande. La seconde écorce des jeunes nches, enlevée au printemps à la dose de 2 à 4 mmes, et la seconde écorce des racines trempée dant une heure dans du vinaigre, peuvent s'emyer comme rubéfiant et vésicant. Dans les cas 1 compliqués de fièvres intermittentes, un braat de cette nature appliqué et bien maintenu au$r$ du poignet trois ou quatre heures avant l'arée présumée de l'accès pourra en prévenir le our par son action révulsive et perturbatrice. On ise la plaie produite par ce vésicatoire avec des illes de noyer enduites d'un corps gras. Quant aux feuilles, leur infusion ou leur décoca (15 à 30 grammes par kilogramme d'eau), ployées extérieurement en lotions, en gargames ou en bains, et administrées à la dose de $q$ à six tasses par jour, semblent le remède par ellence des maladies scrofuleuses : engorgents, ulcères, ophthalmies. L'action du traitement lente, et ne commence guère à se manifester 
avant la fin du premier mois, mais les guéri qu'il obtint sont généralement permanentes, et peut espérer un plein succès dans les trois qu des cas. Ces préparations sont en outre vermif et utiles contre la teigne; elles constituent l des meilleurs lotions astringentes contre les écc ments de mauvaise nature.

On peut remplacer les feuilles par le brou à । double. Le suc du brou de noix, étendu d'eau, rête facilement les diarrhées, et constitue un gargarisme dans les angines chroniques.

VGus le voyez, mes amis, quand même nous vrions réduire à une vingtaine les végétaux ? quels il nous serait permis de demander des mèdes, le noyer occuperait une place d'honn même dans ce petit nombre.

Tout à l'heure, en vous parlant du fruit du No j'ai employé le mot drupe dont je ne m'étais encore servi dans nos causeries. Comme nous trons dans la saison des fruits, et que je ne vor parlé qu'incidemment de cette partie des plan je vais vous donner aujourd'hui quelques not élémentaires sur leur nature et leur classificatio

Une fois que les fleurs ont été fécondées les ganes femelles de reproduction absorbent tout vitalité et la concentrent dans l'ovaire qui c mence à s'accroître sous le nom de péricarp dans l'ovule qui devient la graine : le périć et la graine constituent le fruit. Celui-ci est ou charnu. Parmi les fruits secs, il y en a s'ouvrent pour laisser échapper les graines, on appelle déhiscents, tels sont ceux de l'Aconit la Jusquiame, de la Moutarde; d'autres nom 
ènes sont indéhiscents, c'est-à-dire ne s'ouvrent. s; vous connaissez ceux de l'Angélique, du Pislit, du Persil, et bien d'autres. Si l'akène est ini d'une sorte d'aile membraneuse, comme celui hêtre, il prend le nom de samarre.

Parmi les fruits à péricarpe charnu on distingue drupes, commela Cerise, la Pêche. la Prune, qui

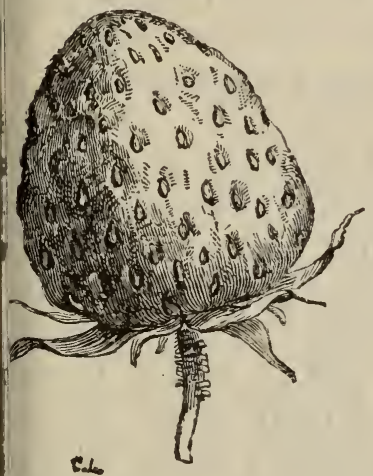

Fraise.

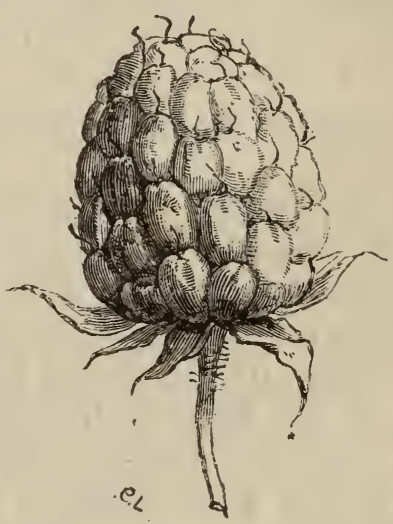

Framboise.

to un noyau, et les baies dont les graines ne sont (s protégées par une enveloppo ligneuse, comme le voit dans le Groseillier, le Cassis. Mais la formation, la structure et l'apparence des its donnent lieu à des phénomènes très-comxes que je ne puis étudier ici avec vous. Ainsi il ive pour quelques-uns que ce n'est pas l'ovaire i devient la partie succulente et comestible, mais réceptacle ou le pédoncule de la fleur. Dans Fraise le réceptacle de la fleur s'accroît, se afle de suc et porte à sa surface les fruits nomux qui sont des akènes, tandis que la Framboiso 
est formée par la réunion de petites drupes br distinctes. Dans la Figue, ce que l'on appelle comr nément les graines sont des akènes produits c cun par une des fleurs nombreuses renfermées di un réceptacle commun qui devient charnu et s culent.

Si je vous faisais un cours de botanique j'en rais ici dans des détails fort intéressants au si des fruits, mais je dois me borner, ainsi que je $\mathrm{v}$ l'ai promis, aux définitions les plus indispensab de crainte de fatiguer votre mémoire et de $v$ éloigner du but pratique de nos promenades.

Vous avez déjà reconnu à la forme papiliona de ses fleurs et à la gousse qui leur succède que ci plante appartient à la famille des Légumineus Elle est remarquable d'ailleurs par ses tiges du. étalées ou couchées, hautes de 40 à 60 centimèt par ses feuilles trifoliées, par sa racine traça très-forte, grosse comme le petit doigt, qui o parfois à la charrue un obstacle assez fort $\mathrm{p}$ l'arrêter, d'où son nom d'Arrête-bœuf. De dista en distance la tige porte do longues épines qui sont autre chose que des rameaux avortés. L'ARri BOEUF (Ononis spinosa) est une plante vivace ce mune dans les terres incultes, les terrains sabl neux; les vaches et les chèvres la broutent, $m$ elle est délaissée par les cochons, les moutons et chevaux; on lui a donné les noms de Bugrane, B grane, Bougrande, Chaupoint, Tenon, Herbe ânes.

On peut arracher en tout temps la racine di l'odeur est désagréable, la saveur douceâtre et $n$ séabonde. Une fois séchéo elle est grise en dehi 
lanche en dedans, et la cassure montre des rayons u centre à la circonférence. La décoction de cette ıcine (30 à 60 grammes par litre d'eau), forme une onne tisane diurétique, utile dans les infiltrations, s engorgements produits par les désordres des ins ou du foie. La décoction très-concentrée des uilles et des fleurs est un bon gargarisme pour s maux de gorge ; elle possède aussi des propriétés urétiques.

La Valériane (Valeriana officinalis), type de la mille des Valérianacées, appelée vulgairement erbe à la meurtrie, Herbe Saint-Georges, Herbe Ix chats, est une plante bisannuelle qui croît dans bois et les lieux humides. Les tiges fistuleuses, nnelées, hautes de 1 mètre à 1 mètre 50 centimèes, sont garnies de feuilles ailées. Elle montre de in à octobre ses jolies cymes en forme de corymbe, mposées de petites fleurs rougeâtres. La seule rtie usitée en médecine est la racine fibreuse, une odeur forte, nauséeuse, d'une saveur âcre et lère, brune en dehors, blanchâtre en dedans. On récolte au printemps avant la pousse des tiges, oisissant de préférence, comme plus active, celle i croît dans un terrain sec.

On administre la poudre de racine de Valériane a dose de 2 à 30 grammes, dans du vin ou mêlée à du el ; l'infusion à la dose do 15 à 30 grammes par e d'eau ; ce sont les préparations les plus usitées. La Valériane a été employée avec succès comme urifuge dans les fièvres d'accès et aussi dans les vres putrides; c'est un vermifuge peu énergique (is nullement à dédaigner; enfin, par son action ctive toute spéciale sur le cerveau et la moello 
épinière, elle est indiquée dans le traitement (Bs) maladies qui dépendent des centres nerveux,

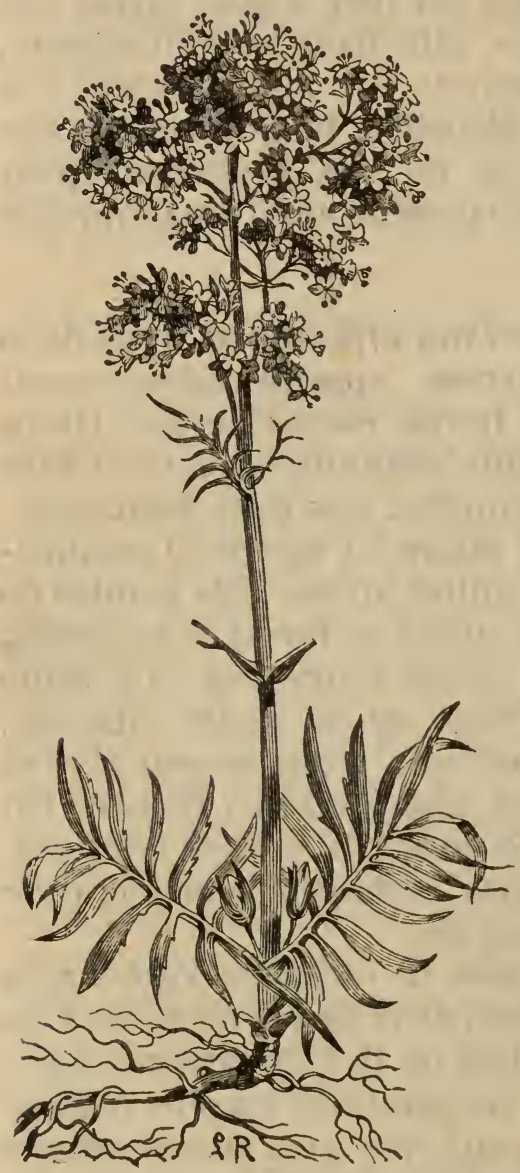

Valériane. principalement lo: qu'il s'agit de ? peurs, maux nerfs, spasmes, compagnés ou $\mathrm{n}$ de battements ou bruits dans la tê de frissons, de bor fées de chaleur visage, d'impatie ces, de brûlemes d'entrailles. $\quad \mathrm{Pc}$ tous ces troubles $\mathrm{n}$ définis de l'organ me, la Valériane d'une utilité ince testable, on peut d même incomparab Elle est douée d'u action excitante système nerveux c se manifeste sur chats comme celle la Cataire, et dd l'empirisme a $t$ très-bon parti cl l'homme.

Quant à ses $\mathrm{p}$ tendues vertus an épileptiques, elles bornent à soulager ou à guérir les convulsions é leptiformes, mais demeure impuissante, com. 
1s les agents connus, contre la vraie épilepsie.

lo nous offre un nouvel exemple des difficultés i entourent l'art de guérir, de l'exactitude rigouise et de l'expérience que réclament les observans sur l'effet thérapeutique des médicaments.

e trouve dans notre cueillette une plante sur uelle je suis bien aise d'avoir l'occasion d'appevotre attention bien que je n'aie pas à vous en seiller l'emploi. Vous la connaissez peut-être s l'un des noms suivants : Plantain aquatique, teau plantagine, Fluteau trigone, Pain de crad, Pain de grenouille. C'est le Plantain d'eau isma plantago), de la famille des Alismacées, te vivace commune au bord des eaux tranquilLes feuilles sont radicales, grandes, entières, les, aiguës, soutenues par de très-longs pétioles tiges hautes de 40 à 50 centimètres so ainent en verticilles qui portent, de juin à sepbre, de nombreuses petites fleurs rosées. o Plantain d'eau a eu ses jours de renomméo : lisait avoir enfin découvert en lui un spécifique ro la rage. Mais uno fois soumise à des expéces méthodiques, cetto plante a failli à sa répuin. L'Allemagne compte une vingtaine d'antiques, la Russie bien plus encore, les Indiens de iérique du sud en vantent plusieurs; chez nous " prôné et abandonné tour à tour l'Aconit, le ron rouge, l'Armoise, l'Eupatoire, le Genêt, la riane, la racine d'Églantier, les cantharides et io la fiento de coucou, les coquilles d'huîtres, personne n'a guéri un cas authentique de rage rrée. Comme je vous l'ai déjà indiqué, ce qui a é et continué les illusions au sujet de la vertu 
de certains médicaments dans cette horrible ma die, c'est qu'il existe une hydrophobie ou horre des boissons qui peut devenir rabique mais de l'origine toute nerveuse n'a rien de commun ay la rage véritable, et qui cède à un traitement ar spasmodique et tonique lorsqu'on l'emploie au dél des accidents.

Remarquons toutefois que les personnes qui la prétention de guérir la rage emploient le $\mathrm{p}$ souvent des reinèdes capables de provoquer transpiration abondante ou obligent le malade à exercice violent. Cela tendrait à expliquer et à c firmer les observations faites sur les bains de peur, seul traitement qui semble avoir réussi or les mains de médecins. Mais puisqu'il existe sorte de rage guérissable, si l'on est en présence d' personne qui se croit menacée d'hydrophobie, on essayer les remèdes populaires connus, ne fût-ce pour agir sur son moral. Quant à la rage elle-mê la succion du venin par des ventouses et la caut sation profonde de la plaie sont seuls capables $c$ prévenir le développement, et contre la maladie clarée, on ne connaît pas de remède, si ce n'est $p$ être le bain de vapeur à une très-haute températ que l'on peut improviser en faisant bouillir l'eau dans une chaudière dans une petite cham

Nous allons inclure dans notre causerie d jourd'hui l'examen de deux plantes qui ne crois pas spontanément en France, mais qui, se trou à la portée de tous, et possédant des propriétés marquables, ont droit, par exception, à entrer । 
tre cadre d'étude. Mon jardin, bien modeste, nous fournit les spécimens.

Parlons d'abord du Pavot (Papaver somnife$m$ ), de la famille des Papavéracées, dans laquelle us connaissons déjà la Chélidoine et le Coquet. Les détails dans lesquels je suis entré au sujet ce dernier m'exemptent de vous décrire longueint le Pavot, qui est un Coquelicot augmenté dans ites ses proportions. Remarquez cependant que feuilles, très-grandes, d'un vert bleu cendré, ne t pas incisées comme celles du Coquelicot. Quant s fleurs, elles sont blanches, pourpres, violettes panachées. La culture a produit des Pavots dous de toutes couleurs sauf le bleu. On les sème en $c \theta$ en février ou mars pour obtenir la floraison même année, ou en septembre pour obtenir la aison en juin et juillet de l'année suivante. On tive aussi une espèce vivace.

l y a deux variétés du Pavot somnifère : le blanc le noir; c'est le blane que l'on emploie de préféce en médecine. Tous deux, originaires de l'Oat, sont cultivés dans le midi de la France pour rs fruits ou capsules destinées aux pharmacies, lans le Nord pour leurs graines dont on retire xile d'œillette.

'odeur du pavot est nauséabonde, sa saveur ère et âcre. Les capsules contiennent une petite intité d'opium, dans lequel résident leurs proj́tés médicinales. Dans les pays orientaux où le rot acquiert des qualités plus actives que sous re climat, on pratique sur les capsules des inciis obliques d'où s'écoule lentement un suc qui raissit en gouttelettes, ce suc séché constitue fium, substance très-complexe dans laquelle les 
chimistes ont découvert une quinzaine de subst ces dont plusieurs et principalement la Morph sont usitées en médecine.

L'opium est le narcotique par excellence : il $\mathrm{F}$ duit graduellement la somnolence, puis le relâc ment complet des forces physiques et intellectuel A dose trop forte il cause des vomissements, mouvements convulsifs, la torpeur et la mort petites doses, il excite et régularise la circulation même temps qu'il calme, émousse la sensibilité $r$ veuse. Cependant il y a des personnes qui ne $\mathrm{p}$ vent en supporter les doses les plus faibles et $c$ lesquelles il agit toujours comme excitant. En s posant à l'élément douleur, à la fois résulta1 cause dans bien des maladies, en régularisan. circulation et par là beaucoup de fonctions, el autres celles de la peau, l'opium est utile dans très-grand nombre de cas, en même temps d'autres médicaments attaquent le mal dans siége spécial ou sa cause déterminée. Il renc grands services dans les maladies nerveuses, affections des organes respiratoires, les coliques diarrhée, la dyssenterie; mais il est contre-indi au commencement des inflammations aiguës, d les fièvres malignes, chez les enfants et chez vieillards.

Il ne faut pas oublier que l'infusion de têtes Pavot ( 1 ou 2 capsules par litre d'eau) contient l'opium et que l'on doit être très-prudent dans l ministration de ce remède. Des nourrices coupa et des mères ignorantes la donnent aux jeunes fants pour leur procurer un calme trompeur; nombreux accidents sont résultés de cette pratic Seul le médecin peut prescrire l'emploi des na: 


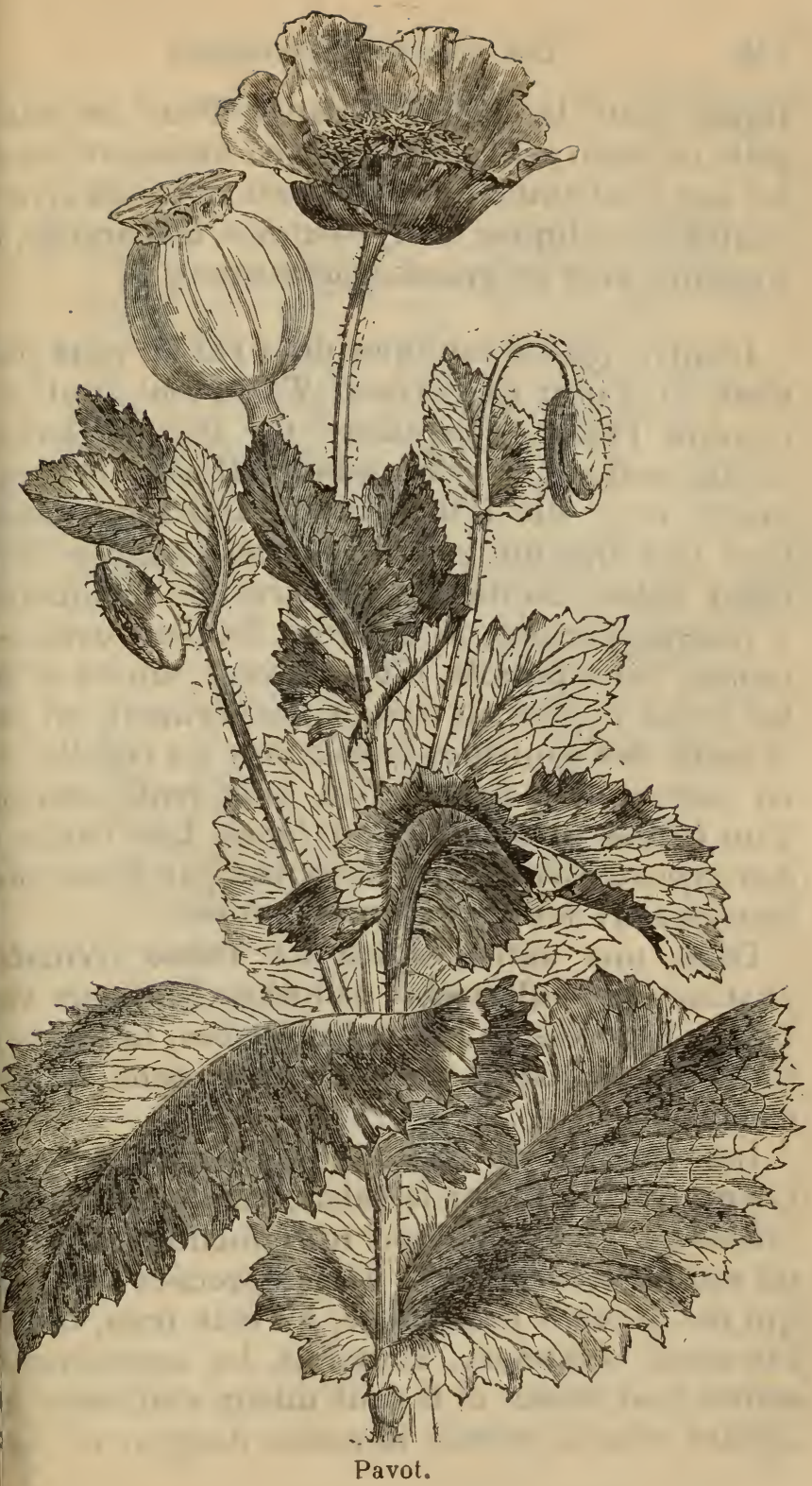


tiques pour les jeunes enfants. Pour les adul cette infusion peut s'employer en fomentations d les cas d'inflammation, d'irritation, ou en lavem, contre les coliques, la dyssenterie, la diarrhée, m toujours avec de grandes précautions.

L'autre plante exotique dont j'ai à vous par c'est le Tabac (Nicotiana Tabacum), qui a nommé Petun, Jusquiame du Pérou, Herbe l'ambassadeur, Herbe sainte, Herbe à tous maux, etc., etc. D'une racine rameuse blanchi part une tige un peu fistuleuse, rameuse, légè ment velue, haute de 1 mètre 50 centimètre 2 mètres, portant de grandes feuilles ovales-l céolées sans pétiole, d'une saveur amère et âc les fleurs rosées ou purpurines forment, en jui et août, des panicules terminales. La corolle, ve en dehors, a la forme d'un tube renflé surmc d'un limbe divisé en cinq lobes. Les fruits \& des capsules ovales qui s'ouvrent par le somme contiennent des graines nombreuses.

Dans une autre espèce de Tabac (Nicoti: rustica) les feuilles sont pétiolées, les fleurs ves tres et disposées en panicules serrées. Le tabac r tique se reproduit naturellement dans beauc d'endroits.

Les Indiens du nouveau monde ne se cont taient pas de fumer le tabac, ils l'employaient médecine, et lors de son introduction en Eurc on soumit cette plante à de nombreuses expérier. qui ont prouvé son utilité, à l'état frais, dans cas assez nombreux. Toutefois, les expériences centes font défaut et il vaut mieux s'adresser à agents mieux connus et moins dangereux. Qu 
au tabac auquel on a fait subir des préparations

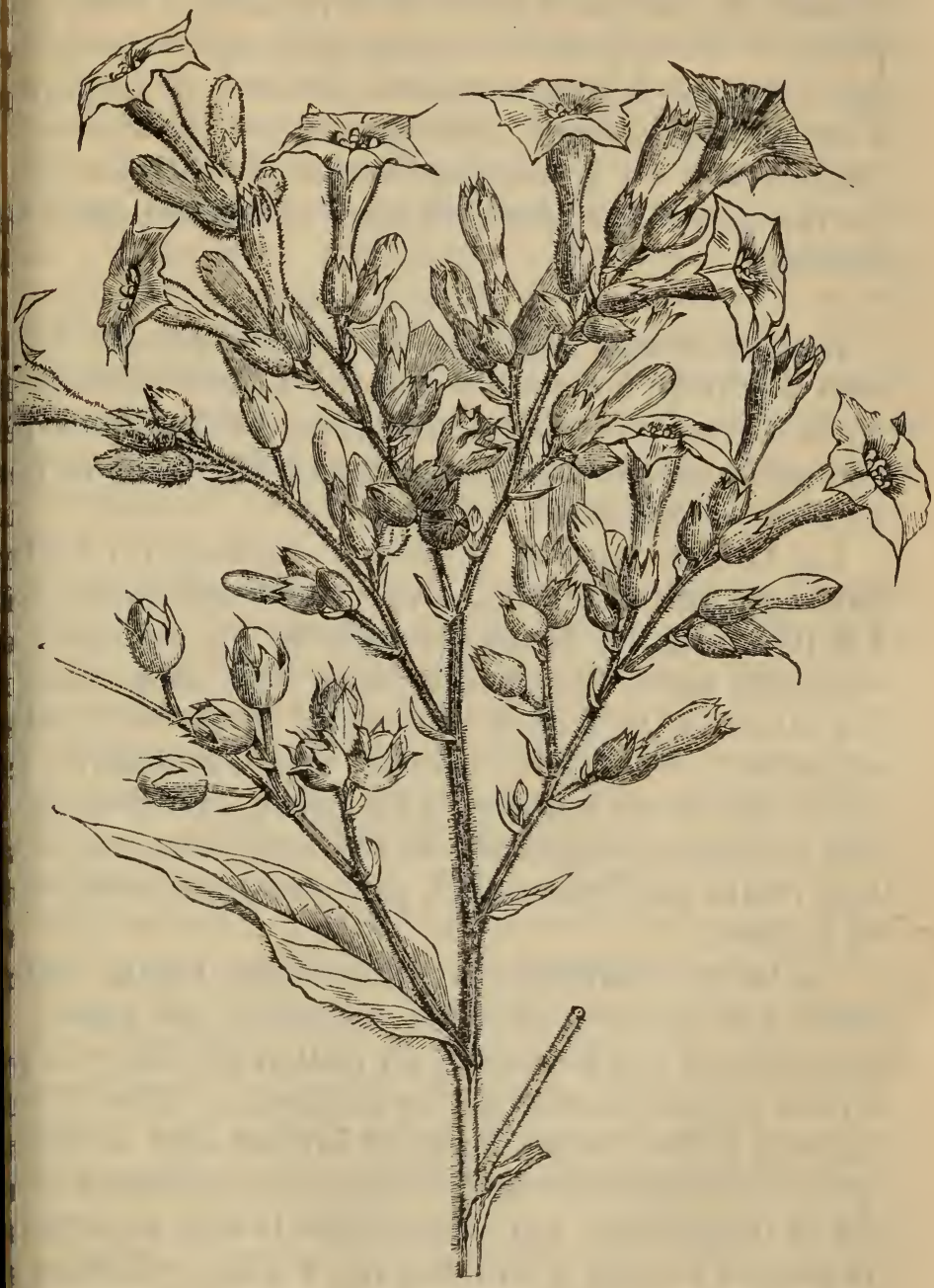

Floraison du Tabac.

péciales pour développer les qualités qui le font 
rechercher des priseurs, des chiqueurs et des $\mathrm{f}$. meurs, je n'ai pas à faire ici le procès de cette pl paration âcre, puante et sale, qui agit intérieuı ment comme poison narcotico-âcre et qui, appliqu à l'extérieur, comme anti-parasitaire, peut encc occasionner de graves accidents. A quelque poi de vue que je me place, je vous conseille donc l's stention.

Revenons aux végétaux de notre pays, qui n'c rien à envier à ceux des contrées lointaines, si n'est d'avoir été l'objet d'études méthodiques $\mathrm{p}$ des personnes compétentes, depuis les derniers pl grès de la mëdecine.

La Laitue vireuse (Lactuca virosa) ou Lait sauvage, Laitue fétide, Lerceron, est une compost La tige, simple, bleuâtre, ramifiée en panicule sommet, porte des feuilles d'un vert bleu cendr les unes, à la partie inférieure, grandes, oval dentelées; les autres, placées vers l'extrémité, F tites, aiguës et incisées. Les fleurs jaunes forme des panicules rameuses à l'extrémité des branch Les fruits sont couronnés par une aigrette soyeu et nacrée.

La laitue sauvage est bisannuelle. On la trou dans les lieux arides, les chemins, les haies, décombres. On la récolte au milieu de l'été, un p avant l'épanouissement de ses fleurs. Elle contic un suc laiteux abondant, de saveur âcre et amè doué de propriétés narcotiques comparables à cel de la jusquiame. On administre le suc exprimé la plante fraîche à la dose de 1 à 60 grammes; l'extrait de ce suc à la dose de 10 centigrammeri 1 gramme. 
Il y a souvent beaucoup d'avantages à substituer à l'opium qui produit la constipation, un calmant légèrement laxatif, et la laitue vireuse rend ainsi de grands services dans les cas d'engorgement des viscères. Elle est également précieuse dans les maladies où l'action excitante de l'opium sur la circulation, tendrait à augmenter une congestion, ou bien lorsque le malade ne peut pas supporter ce médicament, même à faible dose. Le seul inconvénient que présente l'usage des préparations de laitue vireuse, c'est qu'il est difficile de prévoir quelle quantité produira l'effet voulu. On ne doit pas craindre d'ailleurs de commencer par une assez forte dose et de l'augmenter rapidement.

La plante que voici a reçu les noms de Pomme épineuse, Pomme du diable, Pommette, Herbe du diable, Herbe aux sorciers, Chasse-taupe, Endormie. Pour nous, c'est la Stramorne (Datura stramonium), de la famille des Solanées. C'est une plante annuelle qui atteint 1 mètre de hauteur, Elle est remarquable par ses grandes feuilles, d'un vert sombre, molles, anguleuses, à dents aiguës, et ses fleurs olanches ou violettes, en forme de long cornet terniné par cinq dents pointues, correspondant à cinq olis de la corolle. Le fruit est une capsule de la grosseur d'un œuf de poule, d'abord verte, puis prunâtre à maturité, hérissée de fortes pointes, livisée en quatre loges contenant des semences sn forme de rein, noirâtres et chagrinées. Elle croît lans les lieux incultes où l'on récolte ses feuilles 3n juillet. La dessiccation détruit leur odeur et lour saveưr sans nuire à leurs propriétés.

Les feuilles exhalent une odeur vireuse péné- 
trante; leur saveur est âcre, nauséeuse et un pe amère. Son action narcotico-âcre, analogue à cell de la Belladone, est plus irritante. On l'utilise sur tout dans les névralgies, les spasmes et particu lièrement dans l'asthme. Pour toutes les maladie des organes respiratoires, on aspire sa fuméo e fumant des cigarettes préparées avec les feuilles séchées, soit seules, soit mêlées à celles de Sauge; 0 peut aussi bien les fumer dans une pipe.

De même que la Belladone, la Stramoine calm facilement les douleurs, surtout dans les névralgies On administre à cet effet l'infusion ou la décoctio à la dose de 20 à 50 centigrammes par 125 gram mes d'eau. Le même remède s'est montré efficac dans la coqueluche et autres toux nerveuses, dan le rhumatisme aigu ou chronique. A l'extérieur on l'applique en cataplasmes, ou en lotions, seul ou unie à la Jusquiame, sur les engorgements, le tumeurs douloureuses. Les doses ordinaires sor les suivantes : poudre des feuilles de 5 à 30 cent grammes dans les vingt-quatre heures, augmentar. jusqu'à 1 gramme; suc exprimé de 30 centigramme à 1 gramme. A moins d'urgence, on ne l'adminis trera que sur l'avis du médecin, et dans tous le cas il est nécessaire de surveiller les effets de cett solanée et de s'arrêter aussitôt que son actio. physiologique se manifeste par de légers vertige accompagnés de troubles de la vue et de propensio au sommeil. Notons en passant que l'opium nev tralise assez complétement les effets de ce médi cament.

Je viens, mes amis, de vous distribuer des échan tillons d'une plante, commune dans les bois et l 
ong des routes, que vous connaissez sous les noms

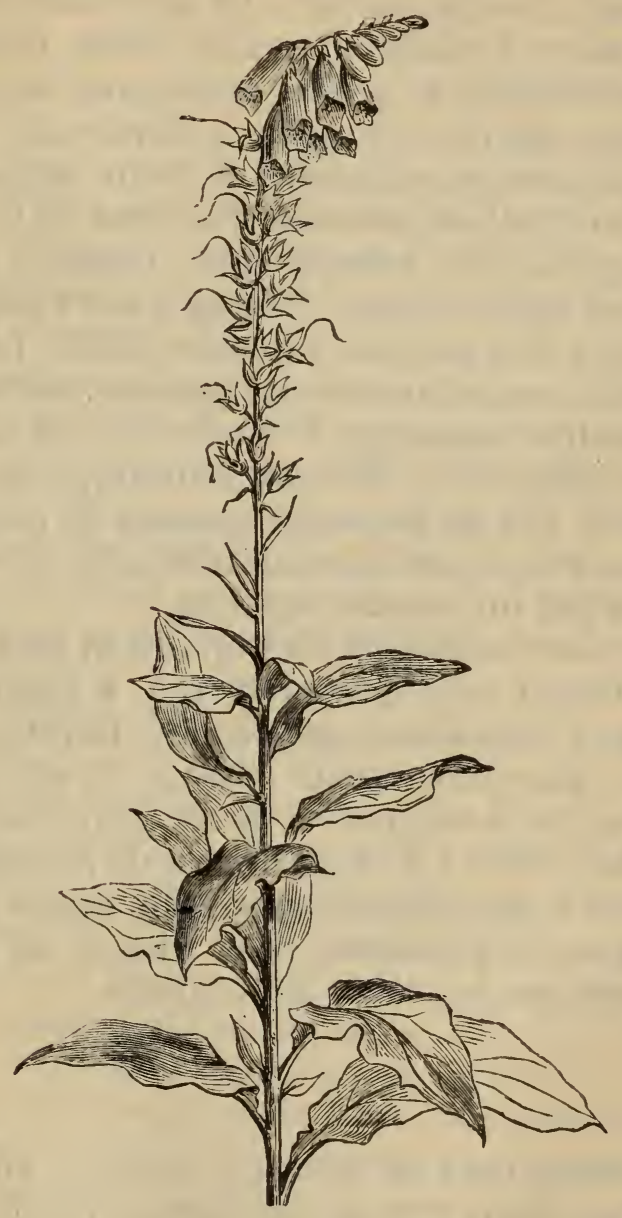

Digitale.

le Gant Notre-Dame, Gantelet, Doigtier, Gaudis, etc. 'est la Digitale (Digitalis purpurea), membre de 
la famille des Scrofulariées dans laquelle nous avon étudié déjà la Véronique. C'est une plante herbacé bisannuelle, haute de 60 à 90 centimètres, à tig simple velue, à feuilles longues, ridées, duveteuse et blanchâtres à la partie inférieure; aux fleur: tubuleuses, garnies de poils à l'intérieur, de cou leur rose pourpre et tigrées de brun, disposées er bel épi terminal et penchées d'un côté de la tige.

La Digitale n'a presque pas d'odeur, mais si saveur est amère et âcre. Elle agit sur l'économie la manière des poisons narcotico-âcres; irrite d'a. bord l'estomac, puis cause des nausées, des vertiges des désordres visuels, de la somnolence et du délire A dose médicinale, elle est diurétique et se fait remarquer par sa propriété spéciale de ralentir les battements de cœur, surtout lorsqu'ils ne sont pas accélérés par un trouble nerveux.

On récolte les feuilles de Digitale en juin et juillet. L'infusion préparée avec 1 à 4 grammes de feuilles par litre d'eau, agit comme diurétique utile dans les hydropisies; pour obtenir un effet spécial sur le cœur et la circulation du sang, l'infusion d'un litre se fait avec 4 à 12 grammes de feuilles, et on l'administre par petites tasses. Son usage réclame la plus grande prudence, et le mieux est de n'en faire usage que sur l'avis du médecin.

En passant près de cette chènevière, vous avez remarqué l'odeur vireuse, pénétrante qu'elle exhale. Par une journée chaude, il serait imprudent de s'endormir dans cette atmosphère qui produit un effet narcotique, dû à l'évaporation d'une huile es- 
ntielle volatile contenue dans les parties vertes u chanvre. Vous avez noté aussi que le ruisseau ans lequel baignaient des paquets de la plante ulait des eaux brunes et qu'il s'en échappait es émanations putrides fort désagréables. Ces iux ont empoisonné les poissons qui s'y trouzient autrefois, et les miasmes qui s'en dégagent int, pour l'homme, une cause de maladie et surut des fièvres intermittentes. Voilà donc une ante qui peut faire beaucoup de mal. Toutefois, ce qui regarde le chanvre, comme dans presque utes les autres circonstances analogues, non seument la somme de bien qu'il peut procurer à 1omme dépasse énormément la somme de mal 'il occasionne parfois, mais l'étude de la nature des causes du danger, puis les progrès de l'inistrie permettent presque toujours d'éviter les sommodités et de mieux profiter des avantages. est facile de ne pas se laisser aller au sommeil ns une chènevière, et quant au rouissage, l'auité désigne pour cette opération certains étangs cours d'eau et l'on peut prendre le soin de ne is habiter dans leur voisinage. D'ailleurs on a u d'espérer que des procédés chimiques et mécayues plus rapides et peut-être moins coûteux, juisant des fibres plus belles, permettront de ioncer entièrement au système actuel. La dispaon des rouissoirs produira, dans certains canis, une amélioration notable de l'état sanitaire.

- Chanvre (Cannabis sativa), originaire des les Orientales, est naturalisé par la culture dans te l'Europe. Il appartient à la famille des Urtiées, dans laquelle nous avons étudié l'Ortie, la iétaire. Ses feuilles sont digitées, c'est-à-dire 
fendues jusqu'au pétiole de manière à former longues folioles étroites. Les fleurs qui s'ouvre en juillet, sont dioïques, c'est-à-dire, vous le save que les fleurs mâles et femelles paraissent s des pieds différents; les mâles forment de petit grappes lâches, composées seulement d'un calice cinq sépales et de cinq étamines; dans les femelle un ovaire surmonté d'un style fendu et velu, $\epsilon$ enveloppé d'une sorte de spathe ou calice d'u. seule pièce. Le fruit est un akène lisse qui re ferme une graine blanche et huileuse.

Lorsque le rouissage a suffisamment désagré le tissu cellulaire qui réunit les fibres dont est fo mée en grande partie l'écorce du chanvre, celui est soumis auteillage, puis au peignage, pour isol et lisser les fibres dont on fait de belles et fort toiles. Le fruit, communément appelé graine, fc recherché des oiseaux, convient à la volaille; on retire une huile employée pour l'éclairage et peinture, dont les tourteaux servent à engraisser bétail.

On cultive dans l'Inde une espèce de Chanv (Cannabis Indica) doué de propriétés plus actir que celui des pays moins chauds, et dont on $\mathrm{pr}$ pare un extrait nommé haschisch, employé $\mathrm{p}$ les Orientaux pour se procurer une excitation cé brale qui se manifeste par une exhilaration accos pagnée le plus souvent d'une sorte de déliro suivie de somnolence et de torpeur. Notre chanv possède à un moindre degré, des propriétés an logues.

L'infusion des feuilles de chanvre (30 à 60 gra: mes par litre) a donné de bons résultats dans rhumatismes chroniques et les dartres; elle ai 


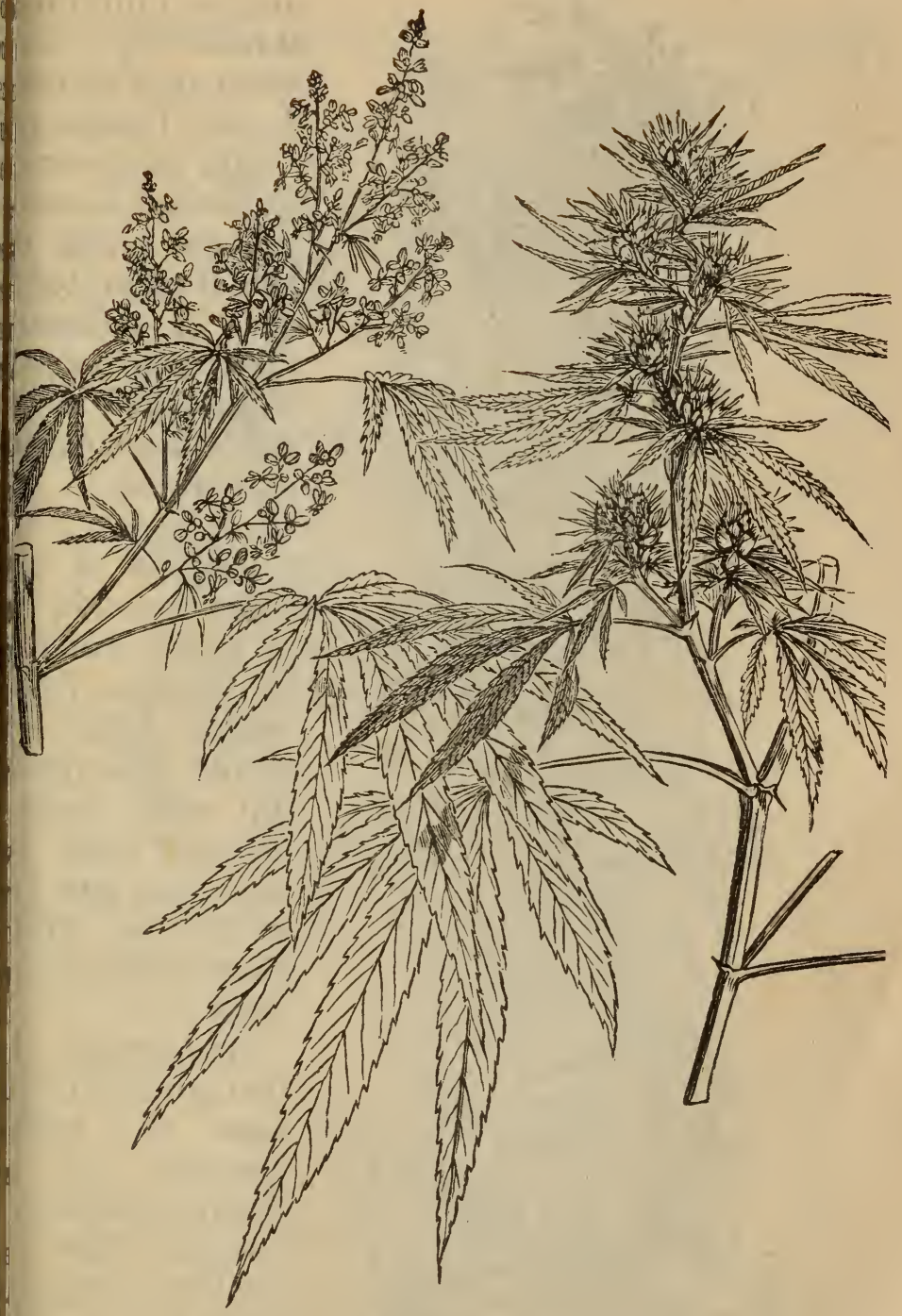

Rameau de Giıanvre mâlẹet femelle. 

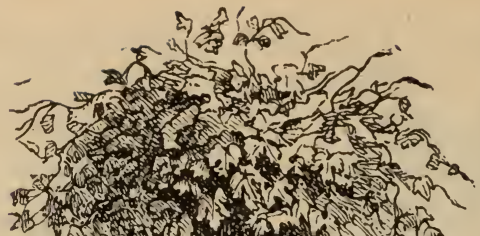

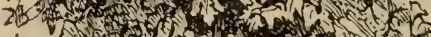

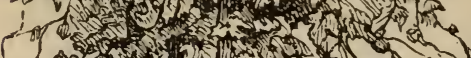

- 5 con

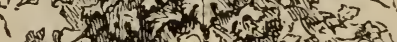

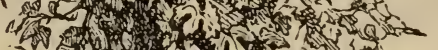
7 . 140.2.

To

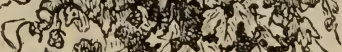
2 s on

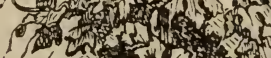

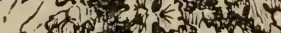

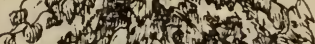
a. 0 mot

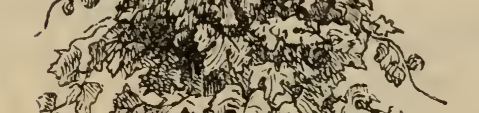
5. rotion $m$ का 1 (1) 1 -

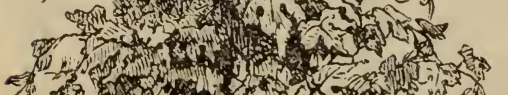

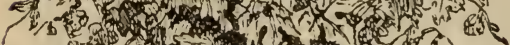

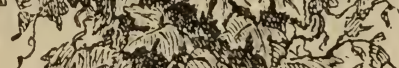
$\{$ (4)

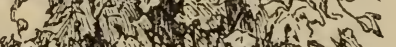
3. 0.2 1 . 4. (3)

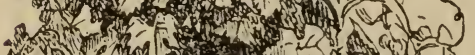

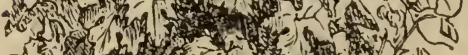
ses 4 s. 15.

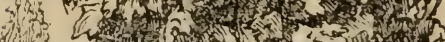

comme diurétiqu etsudorifique.Tor tefois, on s'abstier dra de l'employ, si son usage occ: sionne des nausér ou des maux $c$ tête. Il est probab. que la médecir tirerameilleur pa: ti plus tard de médicamentencos mal étudié.

Passons 'à u autre individu $c$ la même famille le Houblon $\left(\mathrm{H}_{2}\right.$ mulus lupulus plante grimpant qui croît sponts nément dans le haies, et que l'o cultive en gran dans quelques cor trées.

Remarquez l $\epsilon$ fleurs mâles disp sées en petite grappes, et lf fleurs femelles rei fermées dans l $\epsilon$ écailles de ces cônt verts qui passen 2Houblon. 
en mûrissant, au jaune roux, et renferment de petits

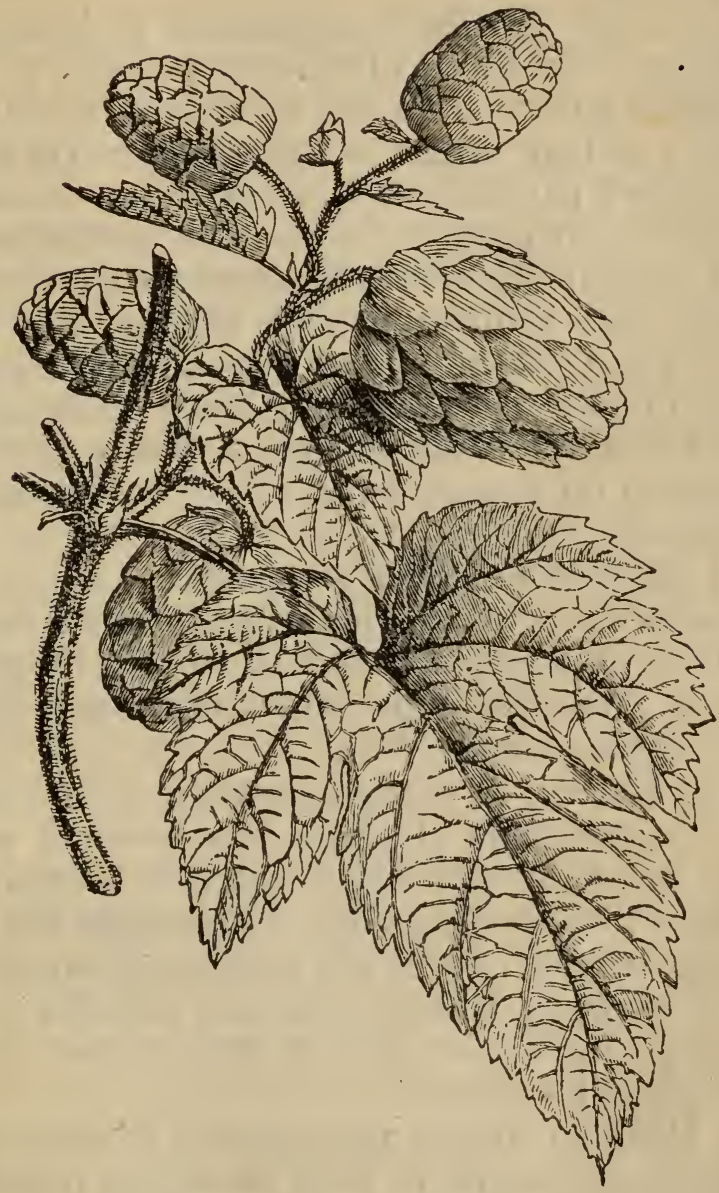

Houblon, cônes florifères.

kènes. Ces écailles laissent exsuder de leur base ne résine odorante et amère nommée lupulin, qui 
possède une partie seulement des propriétés d cône entier.

Les cônes florifères constituent le houblon d commerce. On les récolte à la fin d'août et on le soumet, au four ou à l'étuve, à une dessiccation qu n'altère ni leur arome ni leur saveur. On emploi ces cônes à parfumer la bière et à lui donner l'a mertume peu persistante que l'on recherche dan cette boisson. Mais leur prix élevé les fait remplace souvent par d'autres plantes amères, telle que 1 Trèfle d'eau, l'Absinthe, le Buis, le Quassia qui lv communiquent des propriétés, sinon malfaisantes a moins inutiles, et laissent souvent distinguer dan la boisson un goût âcre fort différent de l'amertum franche du houblon.

On pourrait employer les pointes, les feuilles $\epsilon$ même les sarments de Houblon à préparer de la pe tite bière si le gouvernement autorisait les particu liers à fabriquer pour leur usage cette boisso saine et économique.

Dans le nord, on mange, en guise d'asperges, le jeunes pousses de Houblon; les bestiaux recher chent son feuillage; on en fait des berceaux, de tonnelles; les sarments qui contiennent des fibre tenaces, comme c'est le cas pour beaucoup de plan tes de la même famille, une fois ramollis par $u$ rouissage incomplet, servent à faire des liens for utiles dans les fermes.

Le Houblon mérite une place d'honneur parm les toniques amers, et peut se ranger immédiate ment après la Gentiane. Il n'y a guère de maladi chronique dans laquelle il ne puisse être utile pou relever l'appétit, activer la circulation. Il rend de services du même genre dans les convalescences e 
suffit souvent pour ramener à la santé les personnes affaiblies par de mauvaises conditions hygiéniques: nourriture insuffisante ou trop peu variée, habitation humide, à la condition, bien entendu, de faire cesser d'abord la cause du mal. Dans les affections scrofuleuses, les dartres, le carreau, les tumeurs blanches, les diarrhées chroniques, le scorbut, les engorgements du foie et de la rate, le Houblon est an remède précieux ou un adjuvant utile de la mélication spéciale de chaque maladie. Enfin il est umployé avec succès à haute dose contro les vers ntestinaux, et réussit très-bien dans lo traitement les fièvres automnales après l'usage d'un vomitif et l'un purgatif.

En outre de sọn action remarquable sur la circuation et la nutrition, le Houblon est doué d'une ertu sédative due surtout au lupulin, qui se manieste spécialement sur le système nerveux, et contrijue aux bons résultats de son emploi. La décoction u l'infusion se préparent avec 15 à 60 grammes do ônes par litre d'eau.

Un parfum suavo et pénétrant vient d'attirer otre attention sur cet arbre au port élégant, au euillage touffu, aux fleurs odorantes : c'est le TrLEuL (Tilia Europoea), type de la famille des $\mathrm{Ti}$ iacées, nommé parfois Tillot ou Thé d'Europe. Il roît naturellement dans nos forêts; mais sa beauté 3 fait cultiver dans les parcs et les jardins oú il Jurnit une ample moisson de fleurs d'un blanc jauâtre, disposées en petit corymbe dont le pédonule est soudé en partio à une bractée membraeuse.

On récolte ces fleurs dans les mois de juillet et 
d'août. Il serait bien de séparer les bractées afin de no les employer que dans les cas où l'on désire augmenter l'action diurétique et diaphorétique des fleurs qui sont douées surtout de vertus calmantes et légèrement antispasmodiques. L'infusion des

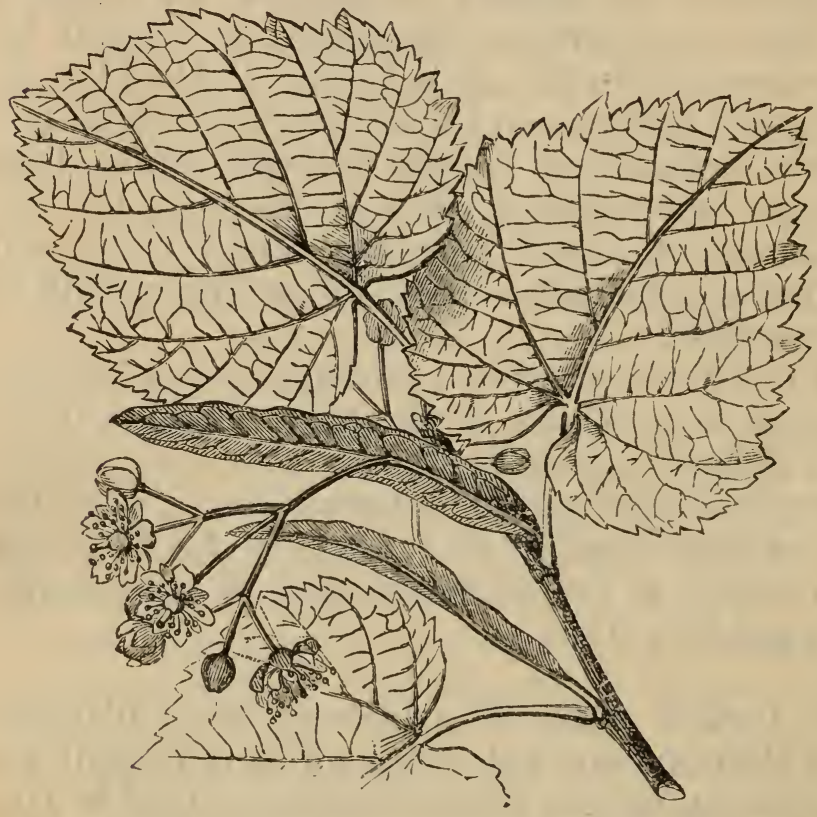

Rameau de Tilleul.

fleurs, d'une odeur et d'un goût agréables, est d'un usage familier dans la migraine, les vomissements, les indigestions, il remplace avantageusement le Thé qui cause souvent une irritation nerveuse. On l'emploie aussi avec avantage dans les diarrhées chroniques, les courbatures, les coliques, les fris- 
ons fébriles. Pour la préparer on emploie de 4 à 0 grammes par litre d'eau.

Pour les diarrhées chroniques dans lesquelles les stringents ne réussissent pas à cause de l'irritaion de la membrane muqueuse qui tapisse l'intesin, on obtient souvent de bons résultats en emloyant avec persévérance un mucilage produit par $\imath$ décoction d'écorce de Tilleul. Cette préparation end aussi de grands services, appliquée sur les laies enflammées, les brûlures. Les feuilles, qui ont aussi mucilagineuses, peuvent remplacer celles e Mauve comme émollient.

La seconde écorce du Tilleul, très-fibreuse, sert n quelques pays à fabriquer des sacs d'emballage, es nattes, des cordes. On l'assouplit aisément par ne simple macération dans l'eau. Son bois, tendre $t$ d'un grain égal, est employé par les layetiers, as sculpteurs, les tourneurs; il donne un charbon ger dont on fait des fusains pour esquisser, et ue l'on emploie en médecine, une fois pulvérisé, omme celui de Peuplier, dans la dyssenterie, la yspepsie. On l'applique aussi comme antiseptique ur les plaies, les brûlures. Ainsi toutes les parties e cet arbre sont utiles, et nous devons le consiérer comme l'une des essences les plus précieuses o nos forêts.

C'est dans mon jardin que nous allons terminer otre promenade. Arrêtons-nous devant cette plante aute de 40 à 60 centimètres à tige herbacée, creuse ; poilue. Remarquez ses grandes feuilles radicales puchées sur la terre et celles de la tige, plus petiis et allongées; toutes sont ridées et velues comme . tige. A l'extrémité des rameaux s'étalent des pa- 
nicules de fleurs quelquefois blanches, mais ordi. nairement purpurines, qui passent au bleu foncé mesure qu'elles s'épanouissent. Le calice form une étoile à cinq rayons sur laquelle s'applique l'é toile bleue de la corolle, dont les pointes se trou. vent placées entre celles des sépales.

Vous connaissez tous cette plante, qui se pro page d'elle-même dans les jardins, et dont les fleur: disputent à celles de la Capucine l'honneur de cou. ronner les salades : c'est la Bourraghe (Borrago officinalis), type de la famille des Borraginées qu: nous a déjà fourni comme sujet d'étude la Grande Consoude; on l'appelle aussi Buglose à larges feuil. les.

On cueille en été les fleuris, et pendant toute 1 belle saison les tiges non ericure fleuries, que l'o fait sécher à l'ombre.

L'infusion des fleurs possède les propriétés de celles de Mauve et de Violette et se prépare de la même manière. La plante contient, au moment $d \epsilon$ la floraison, un mucilage épais dont on utilise les qualités émollientes dans les maladies inflammatoires; pour cela on prépare une décoction avec 30 à 60 grammes de plante par litre d'eau.

Il ne faut pas demander à la Bourrache des propriétés énergiques, pas plus qu'à la Guimauve (Althæa officinalis) ou au Bouillon blang (VerbasCUM THAPSUS) qui doit à ses grandes feuilles blanchâtres, douces et molles, le nom de Molène. Ces remèdes émollients ne sont point appelés à produire des effets capables de frapper l'imagination, mais l'infusion ou la décoction de ces végétaux, dont les fleurs sont légèrement sudorifiques et diurétiques et dont la racine, les tiges ou les feuilles 


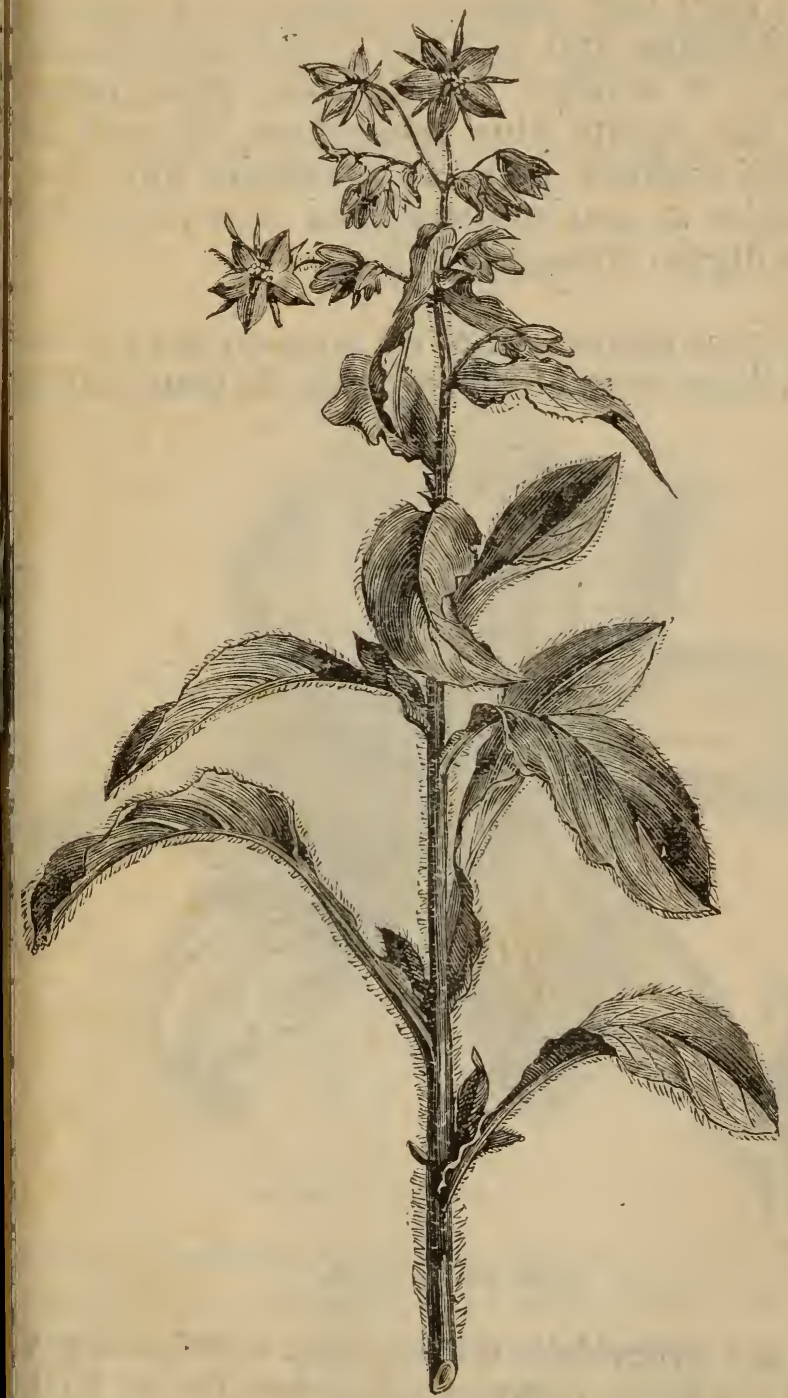

- Buurrache. 
cèdent à l'eau leur mucilage, constituent une res. source précieuse qui suffit à la guérison d'indis. positions, et favorise, dans les cas plus graves l'action des agents plus énergiques. Ce sont les matériaux toujours prêts de breuvages qui, poul être simples et peu coûteux, n'en sont pas moins utiles et dignes d'intérêt.

Examinons encore, avant de nous quitter, ce Lri BLANG (Lilium candidum), et avant de nous enqué

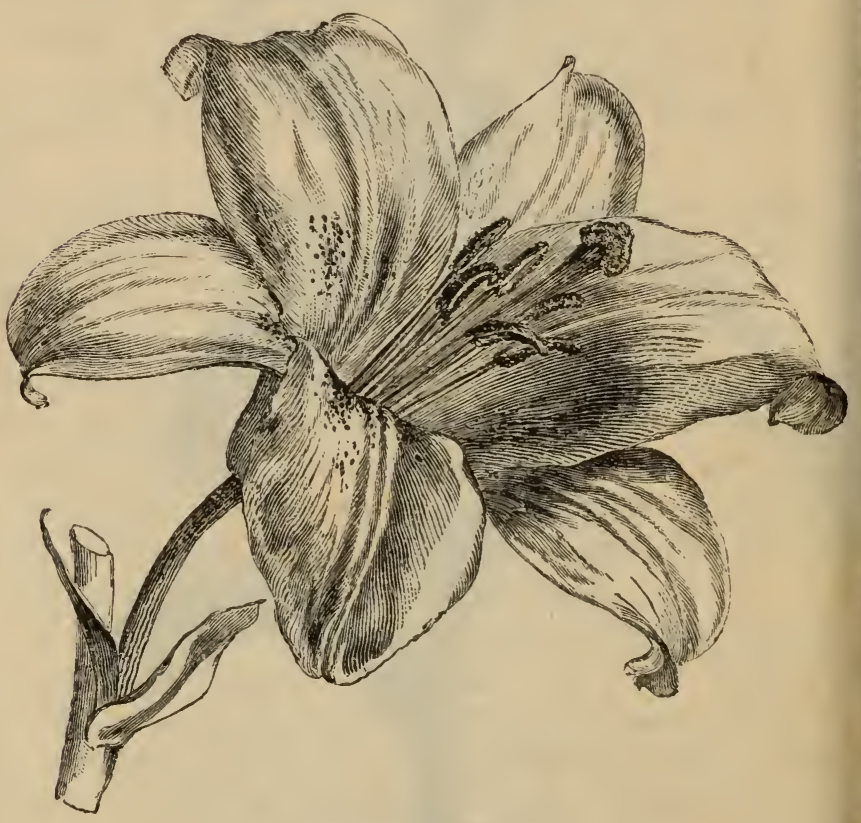

Fleur de Lis hlonc.

rir de ses propriétés médicinales, considérons ur instant les signes distinctifs de la famille des Lilia. 
3, à laquelle il appartient, et dont nous avons dié déjà plusieurs individus : la Scille, l'Ail, perge.

i vous analysez la fleur du Lis, au parfum si

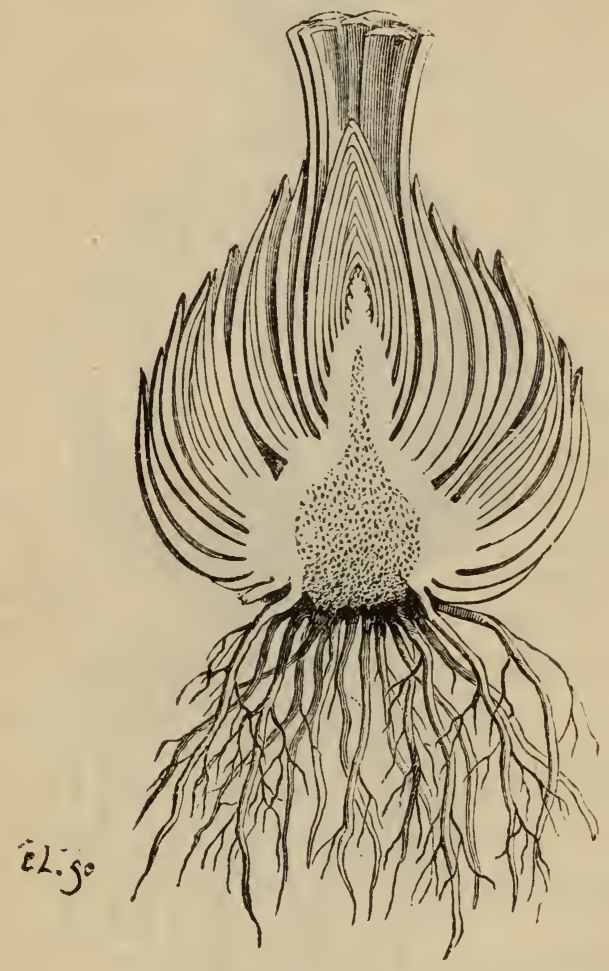

Bulbe de Lis coupe.

e et si pénétrant, vous ne pouvez pas, au precoup d'œil, distinguer les trois pièces du ca des trois autres qui constituent la corolle, car ont toutes une forme et une couleur a peu identiques. Les étamines, très-longues et dé- 
liées, supportent des anthères mobiles qui laiss sortir en s'ouvrant un abondant pollen d'un jar

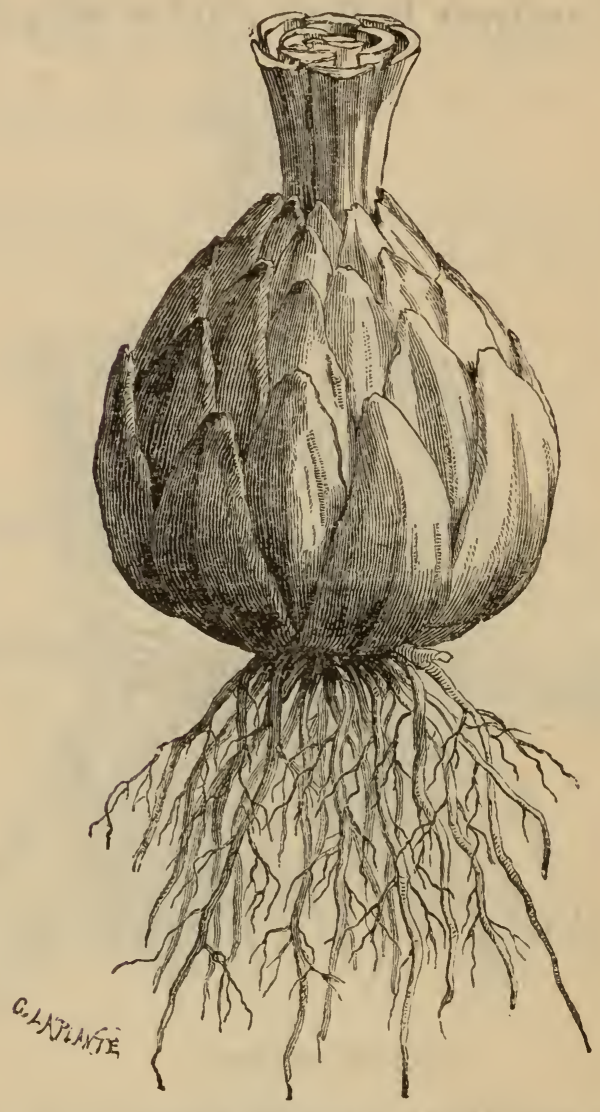

Bulbe de Lis.

vif : le pistil est formé d'un ovaire à trois lo surmonté d'un long style terminé par un stigm. triangulaire. 
La conformation de la tige ornée de petites feuils et supportant des bouquets de fleurs pourrait ire croire qu'au-dessous de cette tige il n'y a que s racines. Cependant si nous arrachons un de ces ants, vous allez voir qu'entre les racines et la ;e aérienne, il y a un organe important : l'ognon, immé en botanique bulbe. Celui-ci est formé par superposition de lames ou écailles disposées auur d'un axe, comme nous le rendons manifeste les arrachant ou en coupant le bulbe par la jitié. De plus, à l'aisselle d'une ou de plusieurs ailles il y a un bourgeon capable de reproduire la inte; de sorte que l'ognon est réellement une tige aterraine, vivace. Dans quelques espèces, l'Asrge par exemple, la forme du bulbe est considéJlement modifiée, mais on y retrouve toujours ; caractères essentiels. Le Poireau, l'Ognon, la lipe, la Jacinthe, appartiennent à la famille qui us occupe; elle peut donc se vanter de fournir à omme des remèdes, des aliments et quelqueses des flours les plus remarquables pour leur rt, leurs couleurs et leur parfum.

Le bulbe de Lis est mucilagineux : bouilli dans ıu ou le lait, ou bien cuit sous la cendre et mêlé u saindoux, on l'emploie comme émollient et turatif sur les furoncles, les engelures, les pa-is, les plaies enflammées.

e Lis, emblème de pureté et de paix, fleurit en ilet. On le multiplie, dans un terrain meuble, osé au midi, soit par graines, soit par les bulles que l'on détache à l'automne des bulbes adulLe Lis bulbifère porte des bulbilles à l'aisselle quelques feuilles de sa tige aérienne. La culture roduit une variété dans laquelle les fleurs sont 
remplacées par un épi de feuilles modifiées de c leur blanche. Il y a un assez grand nombre de riétés de Lis jaunes, rouges, rosés et tigrés, pr que tous assez rustiques pour orner le jardin plus modeste ou même le bord d'une fenêtre.

J'espère, mes amis, que vous vous rappelez explications que je vous ai données au sujet propriétés et de l'administration de quelques-1 de nos toniques amers. En vous reportant à ce je vous ai dit au sujet de la Gentiane, de la Car mille, du Chardon bénit, vous verrez que ces s: ples appartiennent à la même classe de remèc que leur emploi est indiqué dans des circonstan analogues et qu'ils agissent spécialement en ve de leurs principes toniques. J'ai insisté sur leur fluence heureuse dans les états de faiblesse gé rale causés par de mauvaises conditions hygié ques, le manque d'air, de lumière, de nourrit suffisamment abondante ou variée. Vous sa aussi que dans les maladies chroniques et pend la convalescence des maladies aiguës ils prépar l'organisme à l'action des médicaments spécia ou ajoutent à leurs effets, ou rétablissent l'harr nie des fonctions en augmentant la vitalité organes.

En vous signalant comme fébrifuges les amers astringents, j'ai insisté également sur le dan qu'il pourrait y avoir à se méprendre sur les pèces de fièvres dans lesquelles ils donnent de br résultats, sur la manière dont ils opèrent.

Je vais vous parler maintenant de la Petrie C 
URÉE (Gentiana centaurium), de la même famille de la Gentiane, et qui s'en rapproche beaucorp ir ses propriétés. C'est une plante annuelle assez mmune dans les bois, les prés, les terres sablonuses. On l'appelle Herbe au Centaure, Centaulle, Chironée, Fiel de terre, Herbe à la fièvre.

D'une racine blanchâtre, ligneuse, part une tige êle haute de 30 centimètres, entourée d'une rotte de feuilles radicales et garnie d'autres feuilles . us petites ovales-aiguës, terminée, de juin à sepmbre, par un corymbe de petites fleurs roses à rolle tubulée dont le limbe est divisé en cinq rties. On récolte les sommités au plus fort de la raison, on les dessèche rapidement et on les garde ns des cornets de papier pour conserver leurs aleurs et leur saveur d'une amertume intense, i se communique à l'infusion préparée habituelnent avec 10 à 30 grammes de plante par kiloamme d'eau.

Au commencement are son emploi, la Petite ntaurée cause parfois une irritation de l'estomac des intestins. Si cet effet persistait, on devrait 1oncer à son usage car il existerait une inflamtion qui indiquerait le besoin d'émollients et n de toniques.

Dn prépare un bon fébrifuge en faisant infuser grammes de Petite Centaurée et 20 grammes de momille dans un litre d'eau, que l'on donne à dose d'un verre de quatre heures en quatre ures pendant l'accès. N'oubliez pas d'ailleurs que remède et d'autres analogues réussissent tours mieux après l'aảministration d'un vomitif et n purgatif.

I ne faut pas confondre la plante qui nous 
occupe avec la Grande Centaurée (Centaurea $c$ taurium), qui est une Composée, et dont les p priétés sont bien moins prononcées.

Laissez-moi vous mentionner une autre Geni née que la Providence semble avoir répandu dessein près des étangs, dans les marais, $p$ offrir un remède toujours prêt contre les malar paludéennes; c'est le Menyanthe (Menyant trifoliata) ou Trèfle d'eau, excellent tonique, brifuge, vermifuge et antiscorbutique.

Je vais vous dire quelques mots d'une $C$, posée, la Millefeuille (Achillea millefolium), a nommée à cause de ses feuilles longues, un velues, divisées en segments étroits et dent qui lui donnent l'apparence d'une feuille compe à folioles linéaires. Si les noms populaires végétaux pouvaient donner une idée un peu exi de leurs propriétés, celui-ci aurait droit à notre time sans autre examen que la liste de ses titr Herbe aux charpentiers, Herbe aux coupures, $\mathrm{He}$ aux voituriers, Herbe aux militaires, Endove, Sc cil de Vénus, Herbe au cocher, Herbe Saint-Jt La Millefeuille est très-commune dans les li incultes où elle montre, du mois de juin au $n$ d'août, ses capitules de petites fleurs blanches rosées réunis en corymbes à l'extrémité des ramę On la cultive dans les jardins où l'on en trouve । sieurs variétés, mais on doit préférer pour l'us médical, celle qui croît spontanément dans un rain sec.

La racine, trainante, fibreuse, a une odeur $\mathrm{c}$ phrée; la tige et les feuilles, très-peu odoran sont amères et astringentes, tandis que les fle 


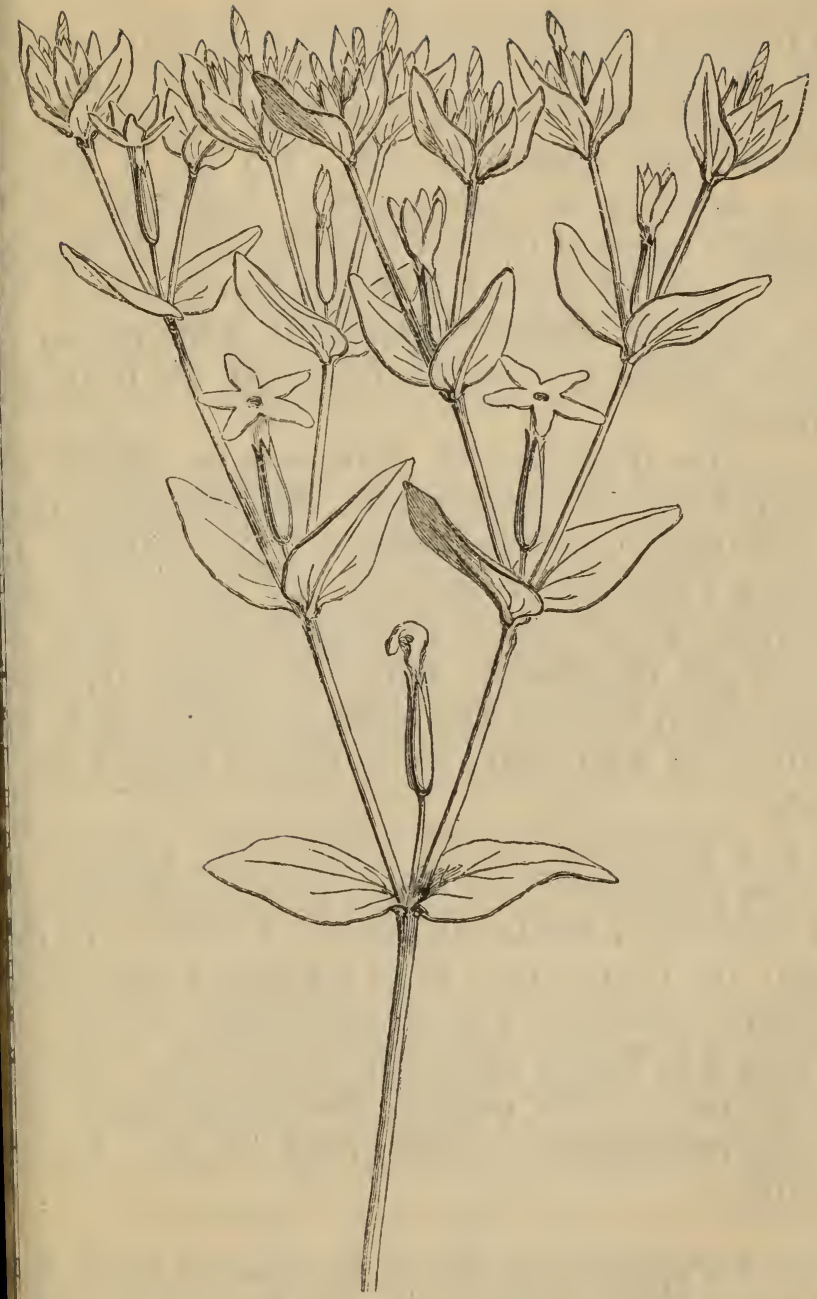

Fleurs de petite Centaurée. 
amères aussi, contiennent un principe aromat que.

L'infusion de Millefeuille, qui se prépare avec à 20 grammes de racine broyée ou de sommit fleuries par 500 grammes d'eau, se décompose $r$. pidement au contact de l'air. On ne la prépa qu'au moment de l'administrer. Les tiges et I sommités agissent comme tonique amer et peuve: être utiles dans les cas très-nombreux que je va vous signaler comme réclamant l'action de c agents, tandis que la racine jouit de propriét excitantes.

Les noms d'Herbe aux charpentiers, Herbe at coupures ont été donnés à la Millefeuille à cau de ses propriétés prétendues cicatrisantes. L paysans l'appliquent souvent, en effet, sur leu coupures, et le travail de cicatrisation, bien qu retardé par la présence d'une substance irritant s'opère selon les lois naturelles, en dépit de la $\mathrm{M}$. lefeuille qui n'en reçoit pas moins le mérite du $r$ sultat.

Il y a plusieurs autres espèces de Millefeuill dont les propriétés sont à peu près semblables : plus énergique est la Millefeuilde norre (Achille atrata) ou Genipi noir dont les fleurs sont jaun. au centre, blanches à la circonférence et qui dc son nom à la bande noire des écailles qui leur se vent de base. Cette espèce, très-aromatique, cro sur les montagnes élevées; c'est un bon toniqu excitant.

Tous les spécimens que vous voyez ici appartier nent à une seule famille, celle des Renonculacées, laquelle nous devons plusieurs de nos remèdes ar 
ifs et aussi de nos poisons les plus dangereux. Elle nous a déjà fourni des purgatifs énergiques: 'Ellébore noir, l'Actée; des rubéfiants et des véicants : la Clématite, la Pulsatille; un antispasmolique : la Pivoine; des altérants : l'Ancolie et surout l'Aconit.

Nous devons nous attendre à trouver, dans les ndividus de cette famille que nous venons de ueillir, des propriétés énergiques, nous rendre ien compte des circonstances qui réclament leur mploi et connaitre la mesure prudente de leur pplication.

Le spécimen que je vous présente ici ne croít pontanément que sur les plages de nos côtes méidionales, mais on le cultive dans les jardins où on repique au printemps les pieds qui ont été sehés à la fin de l'été. C'est une espèce de Pied d'apuette, nommé Staphysaigre (Delphinium Stahysagria), appelée communément Pied d'alouette aphysaigre, Dauphinelle staphysaigre, Herbe aux jux, etc. On n'emploie que les petites graines, brues, ridées, courbées, anguleuses et rudes, connues ins le commerce sous le nom de graine de caucin.

On a administré la poudre de ces graines à l'in rieur, à la dose de 50 centigrammes à 1 gramme, imme éméto-cathartique et anthelmintique, mais n action irritante, capable de produire, à dose un u plus forte, une inflammation mortelle, doit faire jeter absolument son usage interne. On se borra donc à mettre à profit les vertus anti-parasiires de cette graine, pour détruire les poux'ou la le. A cet effet, on emploiera soit la poudre soit le pommade composée d'une partie de poudre 
pour 20 d'axonge, soit une décoction de 15 à : grammes dans un litre d'eau. Il ne faut pas oubli d'ailleurs que la peau, surtout lorsqu'elle est exc. riée, laisse passer dans le sang les substances st lubles avec lesquelles elle se trouve en contact, qu'en appliquant à l'extérieur un remède éners que il est nécessaire d'en surveiller l'effet, car l'a sorption cutanée pourrait produire les mêmes a cidents que l'ingestion dans l'estomac.

Ce que je viens de dire s'applique à cette plan annuelle, commune dans nos moissons où e] montre en juin et en juillet ses panicules de fleu ordinairement bleues, quelquefois blanches ou $r$ sées, et dont la culture a produit des variétés do bles, panachées, qui forment de très-jolis massil C'est le Pied d'alouette (Delphinium consolided nommé aussi Dauphinelle des blés, Consoud Herbe du cardinal. Vous le reconnaissez à s feuilles duveteuses fendues en folioles linéaires, ses fleurs longuement éperonnées.

La plante et les semences possèdent, à un moi dre degré, les propriétés de la Staphysaigre. Da gereux à l'intérieur, le Pied d'alouette, en décolu tion, peut rendre des services contre la gale et maladie pédiculaire; mais il serait imprudent lui demander davantage.

Au premier abord, la plante que voici ressemk assez au Fraisier : c'est la Renoncule aGre (Ranu culus acris), vulgairement Clair bassin, Jaunean Herbe à la tache, Patte de loup, Codron, Grenou. lette, Renoncule des prés, Bouton d'or. D'une raci presque horizontale partent des feuilles palmées 
bes incisés, dentés, et une tige haute de 35 à 60 ntimètres, portant des feuilles de plus en plus roites et des fleurs à cinq pétales d'un beau jaune oré, comme vernissées, supportées par un long et ince pédoncule. Elle croît spontanément dans les ois, les prés, les lieux humides, où elle fleurit au ilieu de l'été. Cultivée dans les jardins, elle proiit le Bouton d'or à fleurs doubles. La plante jouit 3 toute son énergie un peu avant la maturation s fruits. On ne l'emploie qu'à l'état frais, car la ssiccation lui fait perdre ses propriétés, ce qui splique son innocuité mêlée aux fourrages.

Le Bouton d'or préfère une terre un peu forte et imide : on le multiplie par division des touffes au intemps, et l'on a soin de le changer de place tous s deux ans si l'on veut l'empêcher de dégénérer. Avant de vous parler des propriétés de la Renonle âcre, je dois vous signaler quelques autres pèces importantes: la Renongule scélérate (Rainculus sceleratus), nommée aussi Renoncule des arais, Grenouillette d'eau, Herbe sardonique, Mort $x$ vaches, dont les feuilles sont moins découpées, ; fleurs très-petites, et dont les fruits fort nomeux forment une masse ovoïde; la Renongule LBEUSE (Ranunculus bulbosus), aux fleurs trèsandes, aux feuilles plus divisées que celles de spèce précédente, et remarquable par l'expansion lbeuse de sa racine, qui lui a fait donner le nom Rave de Saint-Antoine. Je vous citerai encore la Honcule Thor (Ranunculus thora), qui croît $r$ les hautes montagnes, et dont le suc concentré vait à nos ancêtres pour empoisonner leurs flètes.

Il y a environ cent cinquante espèces de Renon- 
cules, mais celles que je viens de vous signale sont les plus importantes à connaitre au point d vue qui nous occupe. Leurs propriétés médicinale sont à peu près identiques, toutefois il $\mathrm{y}$ en a don le principe actif est plus concentré dans la racin que dans les autres parties; c'est ce que l'on remar que dans la Renoncule bulbeuse.

Les Renoncules ont des propriétés trop âcres trop irritantes, pour qu'on en fasse usage à l'inté rieur. On doit seulement mettre à profit leur actios rubéfiante, vésicante et même caustique, en le appliquant sur la peau dans le but de produire un dérivation ou une substitution salutaire par la quelle on remplace l'irritation d'un organe par cell d'un autre moins important. C'est ainsi que l'or peut prévenir un accès de fièvre au moyen d'us bracelet de Renoncule pilée, guérir ou soulage des rhumatismes, des névralgies, quelques mau: d'yeux, par des sinapismes ou des vésicatoires di même genre. Mais il n'est pas toujours facile d limiter l'action des Renoncules, et chez les per sonnes délicates à peau fine, leur application peu amener la mortification des parties si elle n'est pa l'objet d'une surveillance intelligente. Les feuille de. Bouillon-blanc écrasées constituent le meilleu adoucissant en cas d'accidents de ce genre.

Comme les Renoncules sèches sont inertes, i serait utile de conserver pour l'hiver la teintur préparée avec 1 partie de plantes et 4 parties d'eau de-vie ordinaire ou du vinaigre fort.

Tout ce que je viens de vous dire au sujet de Renoncules s'applique à cette autre espèce do li même famille: l'AnÉmone (Anemone pratensis) 


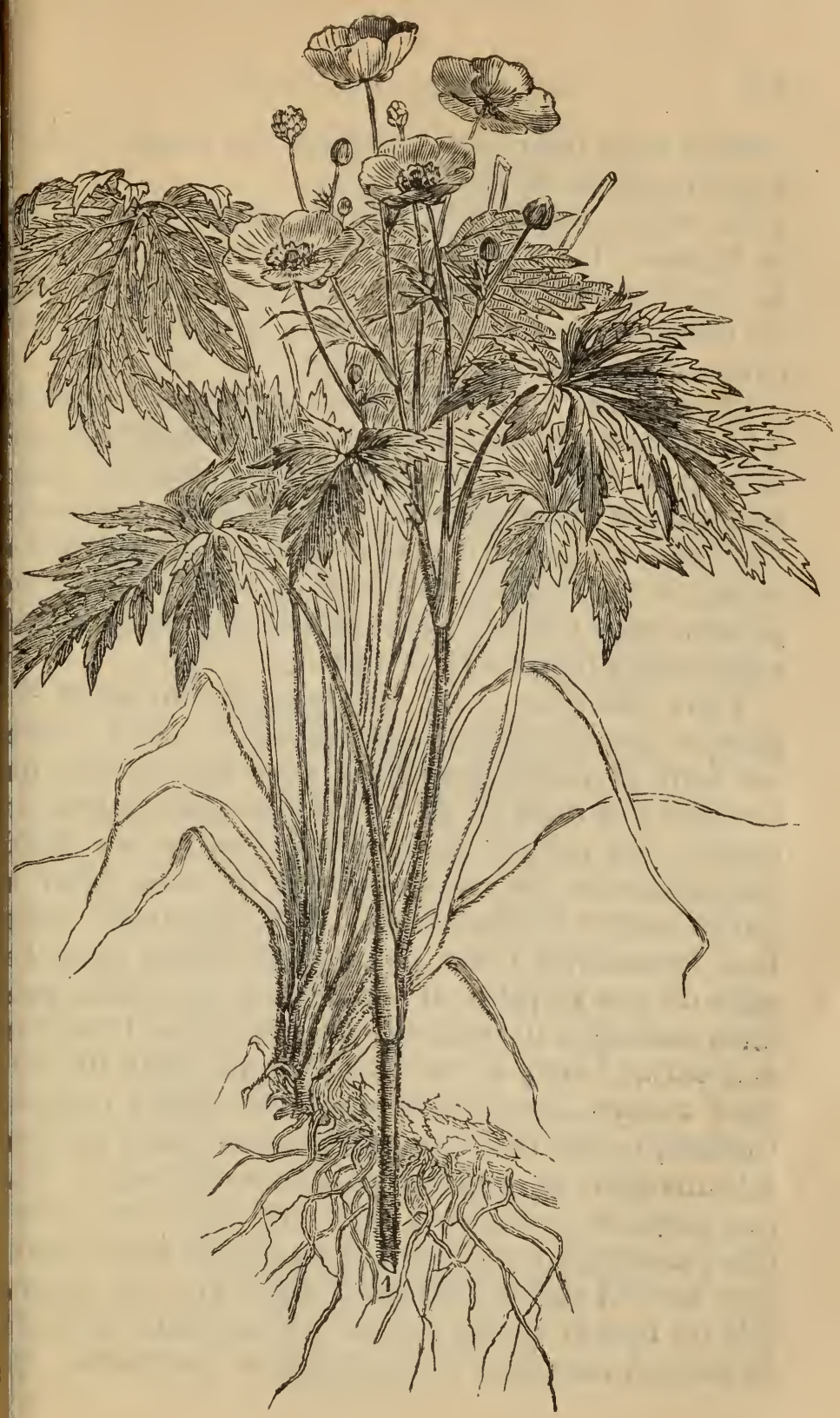

Renoncule âcre. 
connue sous les noms vulgaires de Bassinet, Syl vie, Pulsatille noirâtre.

Sa racine, de la longueur du doigt, est noirâtr et fibreuse; les tiges velues n'ont pas plus de 15 20 centimètres; les feuilles radicales, sont incisée en découpures menues; la fleur est solitaire, pen dante, d'un violet presque noir.

Cette plante est inodore mais très-âcre. Son usag à l'extérieur est indiqué dans les mêmes cas qu ceux pour lesquels on emploie les Renoncules, sujc aux mêmes inconvénients, et réclame la même suı veillance. On peut en préparer une teinture dans 1 vinaigre ou l'eau-de-vie, dont on mouille des com presses pour obtenir l'effet désiré depuis la simpl rubéfaction jusqu'à lá vésication.

Vous entendrez dire quelquefois, au sujet de plantes que je proscris comme médicament intern ou dont je limite les doses avec parcimonie, qu l'emploi des unes ou de fortes doses des autres on amené des cures, sinon merveilleuses, au moin retentissantes. Je vous conseille de vous tenir e: garde contre l'entraînement que de pareils résul tats, fussent-ils vrais, pourraient causer dans l'u sage de nos simples. Il est certain que dans quel ques maladies un remède dit héroïque, l'ancienn médecine Leroy si vous voulez, qui était un pur gatif violent, peut causer dans l'économie une per turbation assez forte pour donner un cours nouvea à la maladie, et susciter une crise favorable. Mai ces moyens énergiques ne sont point du ressor des personnes inexpérimentées. Entre leurs mains leur emploi serait aussi peu sage que de jouer pile ou face la vie du malade : le médecin lui-mêm so permet rarement de semblables hardiesses, qui 


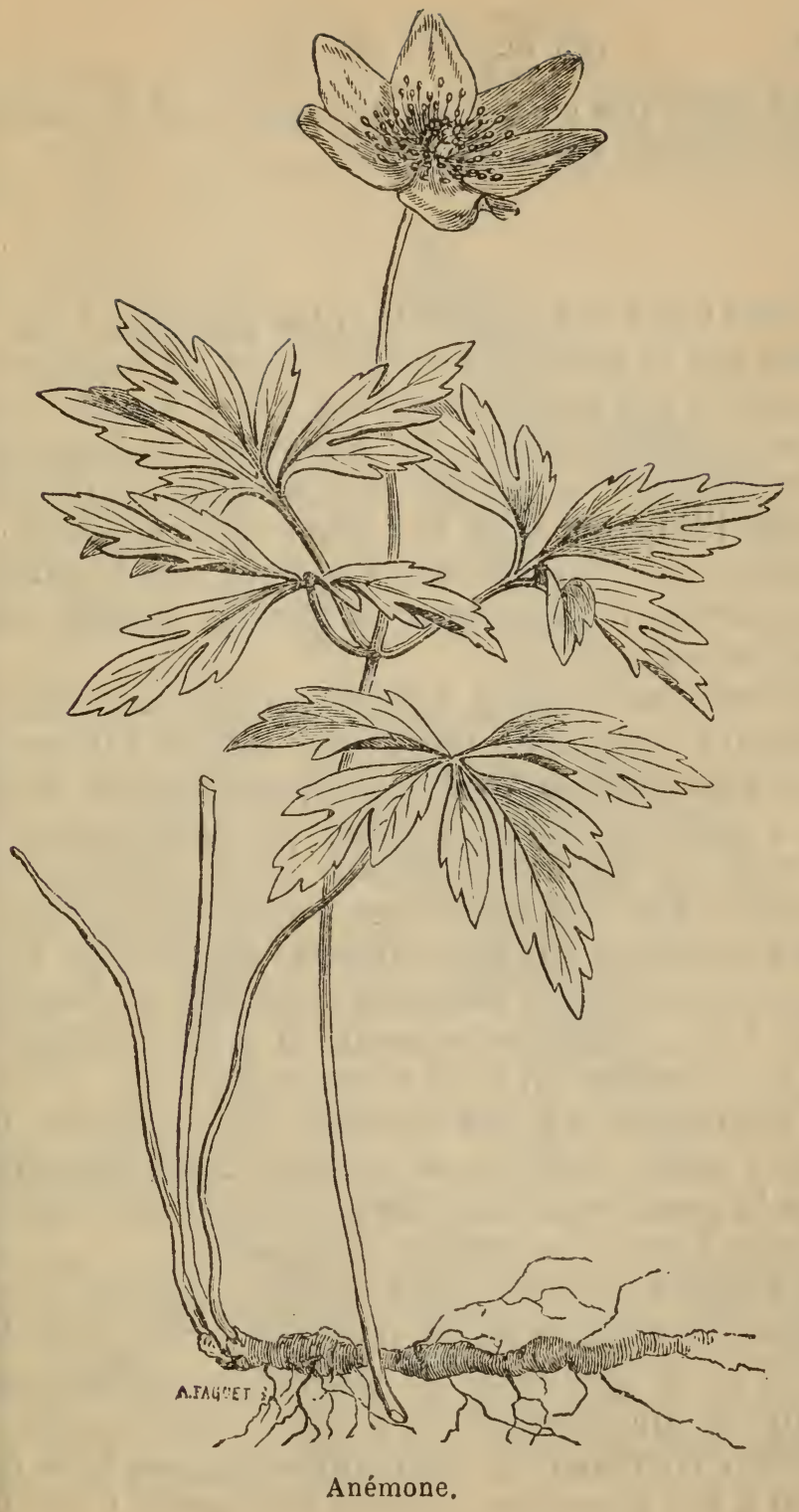


pour n'être pas coupables, ont besoin de la sanction d'une science éprouvée.

Voici l'une des plantes les plus justement recher chées par la médecine usuelle, c'est un membre d la famille des Composées dans laquelle nous avon: trouvé déjà l'Aurone, le Pissenlit, le Tussilage l'Armoise, la Camomille, la Matricaire, le Chardos bénit, l'Eupatoire et la Laitue vireuse. Tous le échantillons que j'ai choisis dans votre moissol d'aujourd'hui appartiennent à cette famille auss nombreuse qu'utile.

Examinons d'abord cette Chiconée sauvage ( $\mathrm{Ci}$. chorium intybus). C'est une plante vivace qu croît abondamment partout. Remarquez'sa racine de la grosseur du doigt, pivotante, brunâtre à l'ex térieur; les longues feuilles à lobes anguleux qu garnissent la base de la tige, tandis que celle-ci e porte de petites qui sont entières; les grandes fleur bleues, quelquefois blanches, solitaires ou disposée deux à deux, sur les rameaux ou à leur extrẻmité qui se montrent en août et septembre.

La Chicorée n'a pas d'odeur, mais possède un saveur amère franche et agréable, plus prononcé dans la racine que dans les feuilles, et qui diminu, beaucoup dans les espèces cultivées.

On donne l'infusion des feuilles fraîches, la dé coction des feuilles sèches ou de la racine, à la dos de 15 à 60 grammes de plante par kilogramm d'eau; lo suc exprimé des feuilles, seul ou uni a suc de Crucifères ou de plantes amères, à la dos de 30 à 120 grammes, comme médicament toniqur 
péritif, laxatif et fébrifuge, dont l'usage, continué ongtemps, est utile, au moins comme adjuvant, ans l'atonie des fonctions digestives, les engorgeients des viscèis, les fièvres léèresdu printemps les maladies roniques do la au.

On cultive en and la chicorée uvage, dans nos partements du ird, pour sa ra.. 1e qui, séchée, rréfiée et réduite poudre, se vend us le nom de ifé chicorée. tte poudre est uvent mêlée, par ude, au café pulu, et pour nstater sa préice il suffit de sser tomber une ccée du mélange spect à la sur-

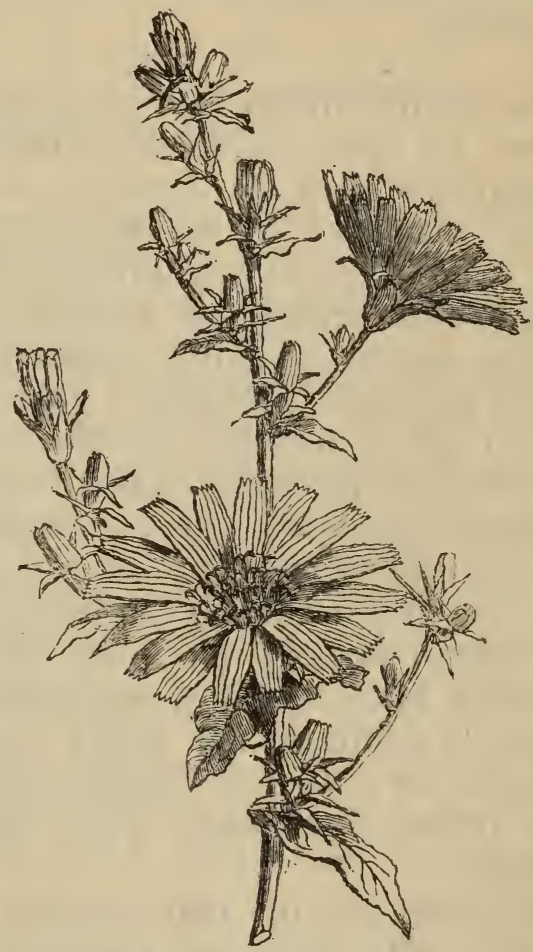

Fleurs de Chicorée sauvage.

e d'un verre d'eau : la chicorée, promptement prégnée par le liquide, tombe au fond et le café ir surnage. La racine, enterrée dans une cave Idant l'hiver, fournit de longues feuilles étiolées, n blanc jaunâtre, que l'on prépare en salade sous rom de Barbe de capucin. 
Cultivée dans les jardins, la chicorée sauvage produit, par des modifications successives, plusieur espèces ou variétés à feuilles planes ou frisées qu l'on mange crues en salade, ou cuites comme le épinards.

La plante vivace que vous voyez ici ressembl assez, en petit, au Soleil ou Hélianthe; elle attein quelquefois près de 2 mètres de hauteur, darss le prés gras, les lieux ombragés, le long des fossés des étangs. C'est l'Aunée (Inula helenium), appelér aussi Inule, Héléniaire, Lionne, Eil de cheval. Lo racine, grosse, charnue, rameuse, d'un jaune bru nâtre en dehors, blanche à l'intérieur, est la seulre partie usitée en médecine. Elle a une saveur amère aromatique, piquante et âcre ; une odeur forte, pé nétrante, agréable, qui, par la dessiccation, se rap proche de l'arome de l'Iris ou de la Violette. Lein feuilles, longues de 30 centimètres et plus, molles crénelées, vertes et ridées en dessus, paraissent, eI dessous, blanchâtres et cotonneuses. En juillet e août se montrent les fleurs terminales, grandes formées de fleurons jaunes, auxquelles succèden des akènes surmontés d'une aigrette d'un blani roussâtre.

L'Aunée est un bon tonique excitant et peut comme telle, favoriser l'expectoration, les sueur ou le cours des urines. On emploie avec avantag l'infusion de 15 à 30 grammes de racine par litrun d'eau dans la faiblesse des organes digestifs, les catarrhes et la diarrhée chroniques, l'asthme hu. mide, dans les cas de débilité générale et dans lest convalescences. Mêlée à de l'eau rouillée, elle es très-utile dans l'anémie, la chlorose et autres ma. 
adies dans lesquelles dominent l'appauvrissement lu sang et la torpeur des fonctions vitales.

La décoction qui est âcre a été employée à l'intéieur contre les dartres, et à l'extérieur contre la ;ale. Pour celle-ci, il vaut mieux appliquer une ommade composée de 2 parties de racine d'Aunée $n$ poudre et 1 partie de saindoux.

Vous avez remarqué souvent sans doute de peites boules couvertes de pointes recourbées qui ?attachent aux vêtements et forment parfois des mas enchevêtrés dans la laine des moutons. En les egardant de près vous reconnaissez que ce sont'les nvolucres ou bases à sépales à demi recouverts is uns par les autres, qui soutiennent les fleurs en apitule de la plante que je viens de faire passer ntre vos mains. La Bardane (Arctium lappa), ommée communément Nupolier, Dogue, Glouron, Herbe aux teigneux, est commune le long es chemins, près des masures, sur les terrains inultes. Sa racine bisannuelle grosse, pivotante, noiitre en dehors, blanche en dedans, inodore, est 'une saveur légèrement amère et astringente. La ge annuelle, heríacée, striée, rameuse, haute o 60 à 90 centimètres, porte de grandes feuilles ès-amères en forme de cœur, crẻnelées, légèrelent cotonneuses en dessous. Les fleurs forment n capitule arrondi de couleur purpurine qui sort un calice globuleux dont chaque sépale se terine par une pointe en crochet; elles sont disposées 1 panicule irrégulière et feuillée.

On récolte en octobre la racine de la première Inée, et celle de la seconde année au commenceent du printemps, mais on peut l'employer fraiche 
en toute saison. La décoction de racine ou $d$ feuilles, préparée avec 15 à 60 grammes de planfle par kilogramme d'eau, est sudorifique, diurétiqu et dépurative. Elle est utile dans le catarrhe pulm naire chronique, la goutte, le rhumatisme, les mile ladies de la peau et les éruptions de mauvai ! nature, à la condition que l'on suive le traitemes pendant un temps suffisant. Elle semble capable ct remplacer la Salsepareille que nous envoie l'Ami? rique intertropicale et qui coûte fort cher. Le sy des feuilles agit favorablement sur les excoriatior superficielles, la teigne, les croûtes de lait. En ar pliquant sur la peau le côté duveteux et glutineu des feuilles, elles causent sur la partie couvert une exhalation analogue à celle produite par le emplâtres de poix de Bourgogne et procurent $u^{4}$ certain soulagement dans les maladies de la po: trine, et les engorgements des articulations.

Mes amis, il me reste à vous parler aujourd'ht de deux plantes qui appartiennent, comme celle dont nous venons de nous occuper, à la famille de Composées et qui se ressemblent beaucoup pa leurs propriétés. Je passerai rapidement sur la pre mière et vous donnerai plus de détails sur la se conde qui est plus énergique.

La TANaisie (Tanacetum vulgare) est une plant vivace qui croît spontanément dans les terrains in cultes et un peu frais, le long des chemins, dan les prairies, et qui produit, par la culture, une bell variété à feuilles frisées. Seuls les moutons man gent ses feuilles desséchées. La plante étendue er litière dans la niche des chiens les délivrent $d c^{\mathrm{t}}$ leurs puces. Vous avez pu l'entendre désigner sous 
s noms de Barbotine indigène, Herbe aux vers, lerbe Saint-Marc. Elle est facilement reconnaisable à ses feuilles incisées et découpées en lanières roites, dentées; à ses fleurs jaunes qui forment, e juillet à septembre, des corymbes très-compactes l'extrémité des rameaux. Ces fleurs sont composées une agglomération de petits fleurons tubulaires ès-courts auxquels succèdent des akènes. Ceux-ci administrent comme vermifuge à la dose de 2 8 grammes en poudre incorporée à du miel, ou élayée dans du vin.

On cultive la Tanaisie dans une terre franche, blonneuse et fraîche, bien exposée : on sème en ace au printemps ou en pépinière à l'automne; on ut aussi la multiplier par éclats de pied détachés l commencement du printemps. On récolte au ois d'août ses fleurs dont l'odeur est forte et péstrante, la sáveur aromatique, nauséeuse, trèsnère, et qui ne perdent rien de leurs propriétés ir la dessiccation. Leur infusion à la dose de 15 à grammes par litre d'eau, est tonique, excitante, rmifuge, anti-fébrile et convient aussi dans les cidents nerveux, les spasmes.

Appliquée en cataplasme sur le bas-ventre, seule l mêlée d'ail, la Tanaisie agit comme vermifuge; décnction concentrée est un bon détersif, stimunt et antiseptique des plaies de mauvaise nature.

Nous allons terminer notre causerie par l'étude - l'une de nos plantes les plus précieuses : l'ABvтне (Artemisia absinthium), nommée aussi fande absinthe, Absin menu, Alvuine, Aluine, cmoise amère, Herbe sainte, Herbe aux vers. C'est une plante herbacée vivace qui se propage 
d'elle-même dans les lieux incultes, et que l'on cl tive dans une terre légère exposée au soleil. On sème au printemps ou l'on plante, à cette époq des éclats de vieilles souches. En hiver il faut l'e tourer d'un paillis.

D'une racine ramifiée part une tige droite, du! cannelée, rameuse, d'un gris cendré, qui atteint 60 à 70 centimètres et porte des feuilles décomp sées finement découpées, soyeuses sur les der faces, d'un blanc argenté en dessous. De juillet. septembre s'ouvrent les petites fleurs globuleuse jaunâtres, formées par l'agglomération de peti fleurons tubulés et disposés en panicule allongé

On récolte, à l'époque de la floraison, les fleu et les sommités, que l'on fait sécher à l'ombre; ell conservent après la dessiccation leur odeur fort aromatique et leur saveur très-amère. Elles cor tiennent une huile essentielle volatile qui s'évapo: pendant la préparation du décocté de la plante reste dans l'infusion en vase clos; puis des prir cipes résineux amers moins excitants et plus ton ques que l'huile essentielle. La poudre, le vin, teinture d'absinthe contiennent les deux classes $c$ principes.

La poudre, à la dose de 4 à 16 grammes, le vi (1 partie de plante pour 30 de vin blanc) à la dos de 30 à 125 grammes, sont fort utiles comme fébr fuges surtout dans les régions marécageuses et che les sujets débilités atteints d'engorgement dè 1 rate, de décoloration et d'infiltration de la pear Une fois la fièvre coupée, on continue l'administra tion journalière du médicament à petites doses pour mettre simplement à profit ses propriété toniques, stimulantes et diurétiques. 
Le vin blanc dans lequel on fera infuser 30 gram-

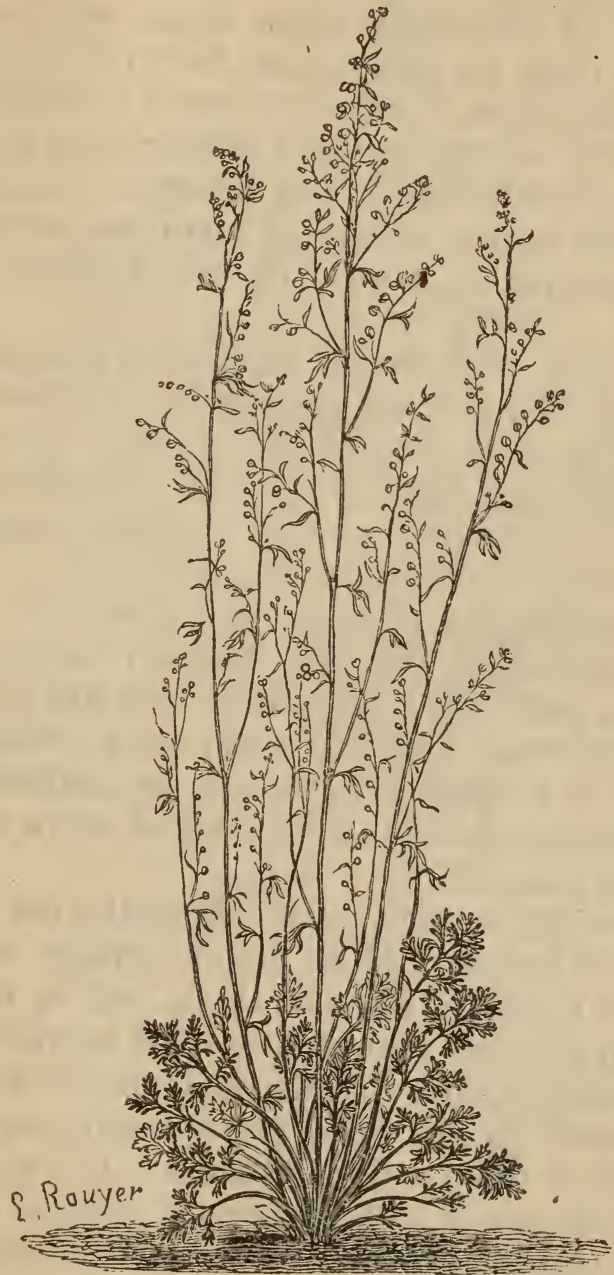

Absinthe.

d'Ail et 30 grammes d'Absinthe par litre, pris 2e partie. 
à la dose de 30 à 100 grammes par jour, est un excel lent fébrifuge et vermifuge. Le vin préparé ave l'Absinthe et l'écorce de saule blanc remplace ave avantage, dans les campagnes, le vin de quinquin: Enfin la décoction d'Absinthe, seule ou additionné de sel marin, est un excellent antiseptique des plaie et ulcères, qu'elle assainit et cicatrise promptemer lorsque son action locale est favorisée par un tra tement général approprié et une hygiène convı nable.

C'est avec différentes espèces d'Absinthe d Alpes, que l'on prépare la liqueur nommée Crèn d'Absinthe ou Absinthe suisse; on y ajoute por l'aromatiser de l'Angélique et de la Badiane. Cet liqueur ne renferme que le principe excitant l'Absinthe, l'huile essentielle, qui agit à peu pr à la manière des poisons narcotico-âcres. Aus l'excès accidentel de cette boisson cause-t-il ul excitation générale accompagnée de soif et de ch leur à l'estomac, tandis que son abus prolongé o casionne la stupeur, l'hébétude, les hallucination l'affaiblissement de l'intelligence, et enfin un abr tissement complet.

Vous le voyez, mes amis, l'Absinthe est à la fc un remède précieux et un agent terrible, selon l' sage que nous en faisons. Il en est de même beaucoup d'autres plantes que nous avons étudiée La Providence, en permettant à l'homme d'analys les richesses qu'elle étend partout sous ses pas, a imposé le devoir d'appliquer à la fois son intel gence et sa raison à discerner en chaque chose part du bien et du mal. Il ne reçoit point les do de la nature sous une forme qui encourage sa $\mathrm{m}$ lesse et son oisiveté, mais bien sous celles qui 


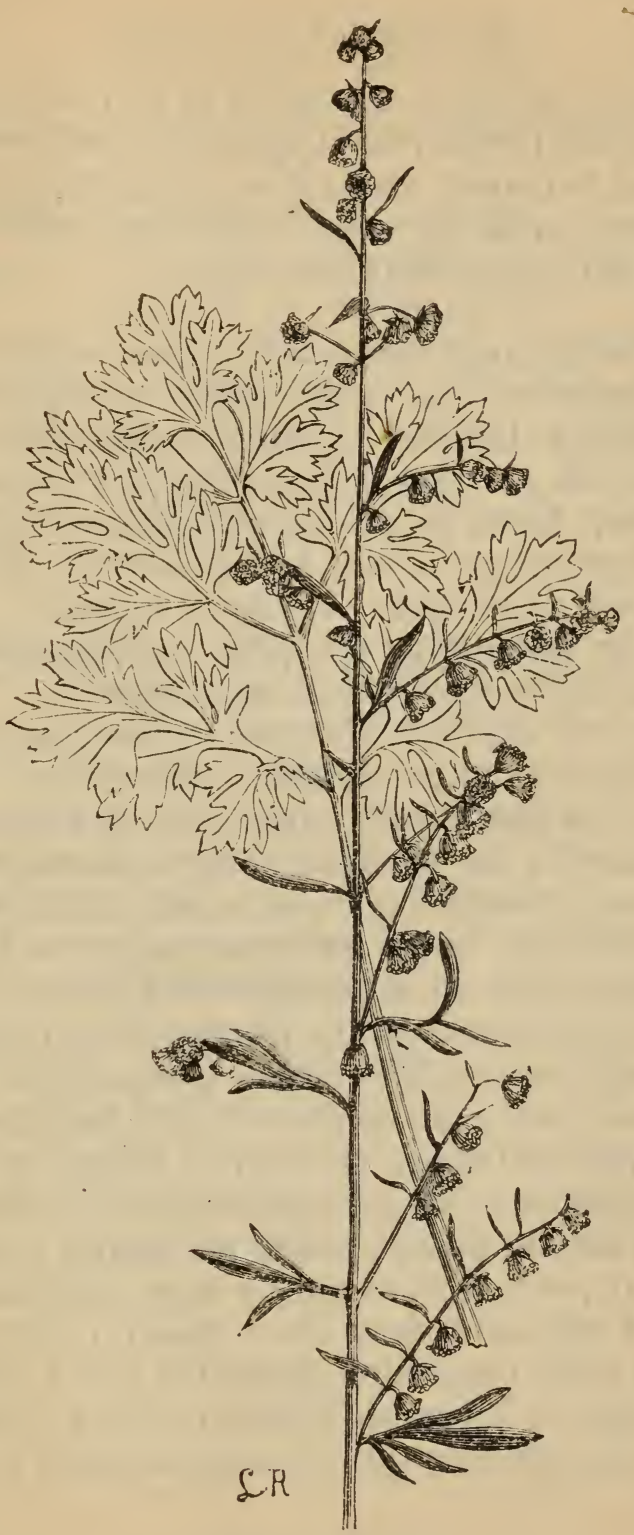

Sommité d'Absinthe. 
permettent le mieux d'exercer et de développer ses facultés, de fortifier et d'appliquer ses vertus natives, par le travail, l'attention, le jugement, la persévérance, sans lesquels il passerait sur la terre comme les créatures inférieures.

Nous terminons aujourd'hui, mes amis, la série de promenades que nous avons entreprises pour vous donner le goût d'étudier nos remèdes des champs, nos simples, et aussi pour vous indiquer brièvement leurs propriétés les plus saillantes. J'ai profité également de ces herborisations pratiques pour vous apprendre, à bâtons rompus, les éléments de Botanique les plus indispensables au point de vue spécial de nos causeries.

Pour être complet, j'aurais eu beaucoup plus à vous dire sur la nature des maladies et des re- 1 mèdes; il m'aurait fallu entrer dans des détails plus circonstanciés au sujet de chaque plante et ouvrir à beaucoup de celles que j'ai omises le cadre de mes : leçons. Mais le temps, les circonstances et le but de nos promenades ne le permettaient point. Je crains même que poul beaucoup d'entre vous notre tâche restreinte n'ait semblé un peu compliquée. A ceuxlà je vais donner un conseil. Parmi les plantes que nous avons étudiées, il y en a beaucoup qui ont des propriétés à peu près identiques : au lieu de les étudier toutes, limitez-vous, au moins provisoire-s ment, au petit nombre que je vais vous énumérer. Les bien connaitre sera pour vous un fonds excellent qui vous permettra d'étendre peu à peu votre étude selon le temps que vous pourrez y consacrer et les circonstances qui vous appelleront; à en faire usage. 
Liste des plantes les plus importantes à connaître :

Chiendent. - Citrouille. - Thym. - Anis. Colchique. - Bryone. - Gentiane. - Pariétaire. Chêne. - Valériane. - Saule blanc. - Muguet. - Sureau. - Clématite. - Aconit. - Raifort sauvage. - Ail. - Camomille. - Belladone. - Alleluia. - Menthe poivrée. - Persil. - Coloquinte. - Noyer. - Pavot. - Digitale. - Houblon. Moutarde. - Renoncule. - Chicorée sauvage. Absinthe.

Un dernier mot avant de nous séparer. Je vous ai, dès le premier jour, expliqué le but de nos promenades. Vous savez que j'ai voulu seulement vous mettre à même, en l'absence de médecin, de soulager les malades et d'exercer au chevet du pauvre un ministère de charité. Laissez-moi vous affirmer avec une expérience qui date déjà de loin : quelle que soit la peine que vous vous donniez pour acquérir les connaissances nécessaires, quels que soient les sacrifices que vous vous imposiez pour les appliquer, vous serez amplement dédommagés par la conscience d'être devenus plus utiles et par la joie de rendre à la santé, à la vie, un proche, un $\mathrm{ami}$, ou quelque déshérité dont le remerciement sera pour vous la meilleure, la plus complète récompense. 


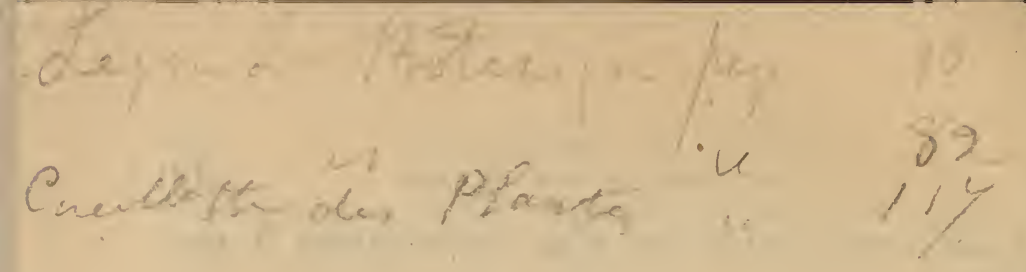

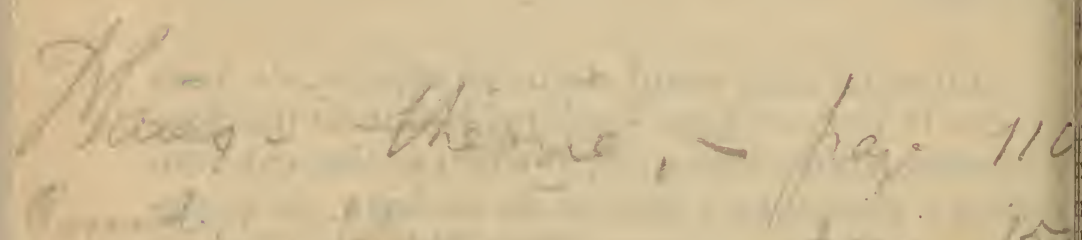

$$
\begin{aligned}
& \text { tor the sig }
\end{aligned}
$$

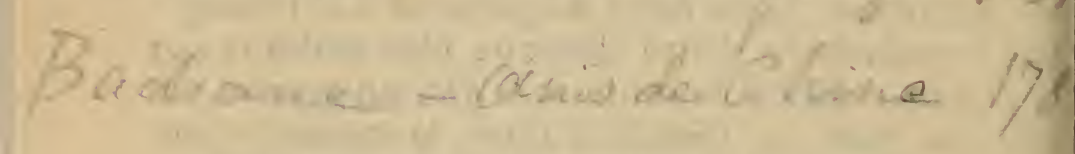




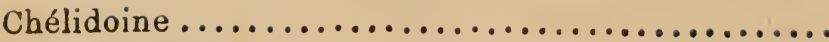
Chèrefeuille ...........................

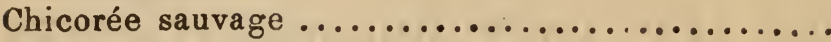
Ciguë Clématite des haies.

Cochléaria

Coloquinte Coquelicot

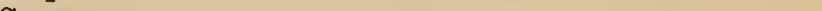

Cresson .............................

Digitale.

Eglantier.

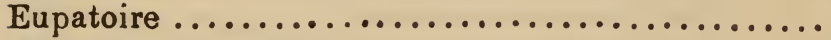

Euphorbe épurge ........................ Fraisier.

Fréne

Fumeterre

Genêt à balai

Géranion.

Germandrée maritime

Gratiole.

Houblon

Hyssope

Iris commun

Jusquiame.

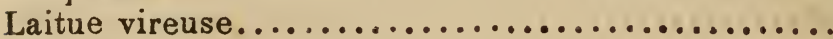

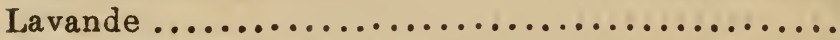

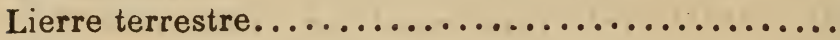

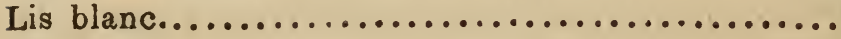

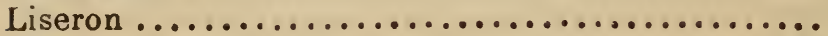

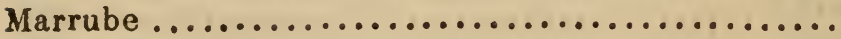

Matricaire.................................. Mauve

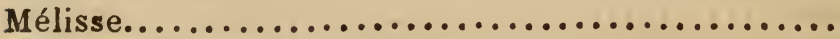

Menthe poivrée.............................

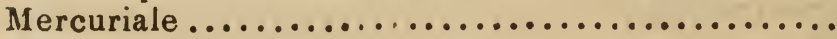
Millefeuille.............................. Momordique ............................

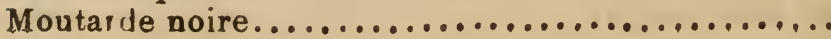


iguet...............................

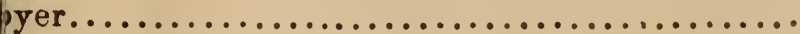

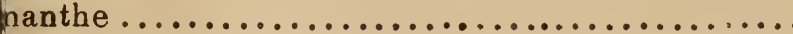

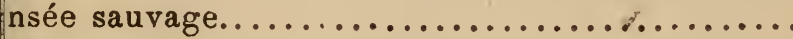

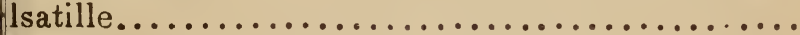

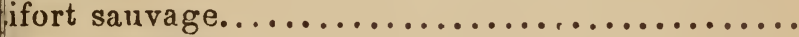

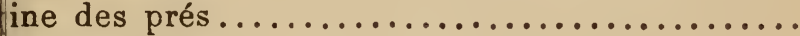

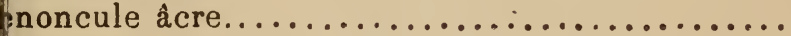

rriette $\ldots \ldots \ldots \ldots \ldots \ldots \ldots$

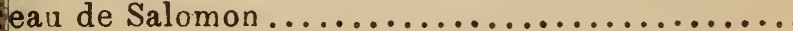


Coulommiers. - Typ. Paul BRODARD et Gie. 





\section{Date Due}

All library items are subject to recall at any time.

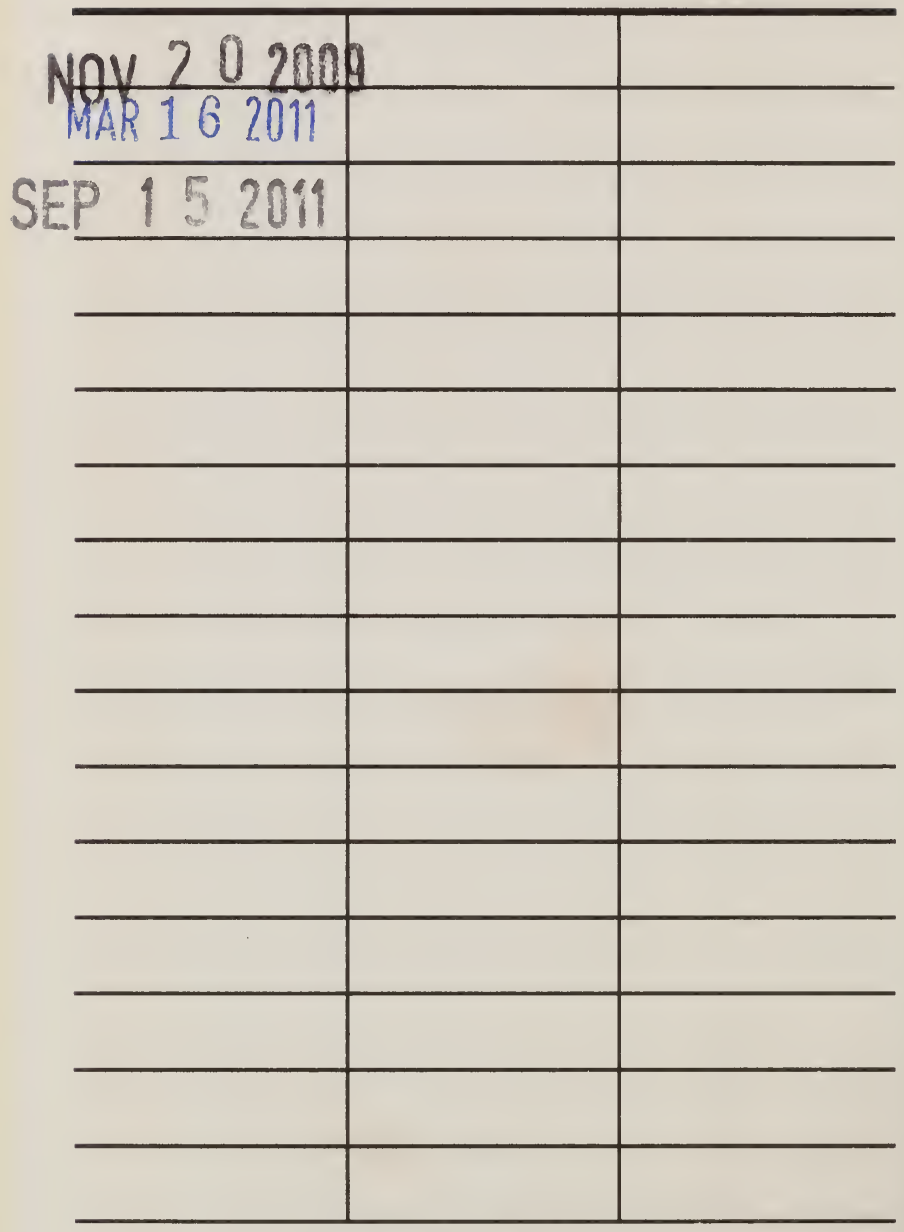

Brigham Young University 

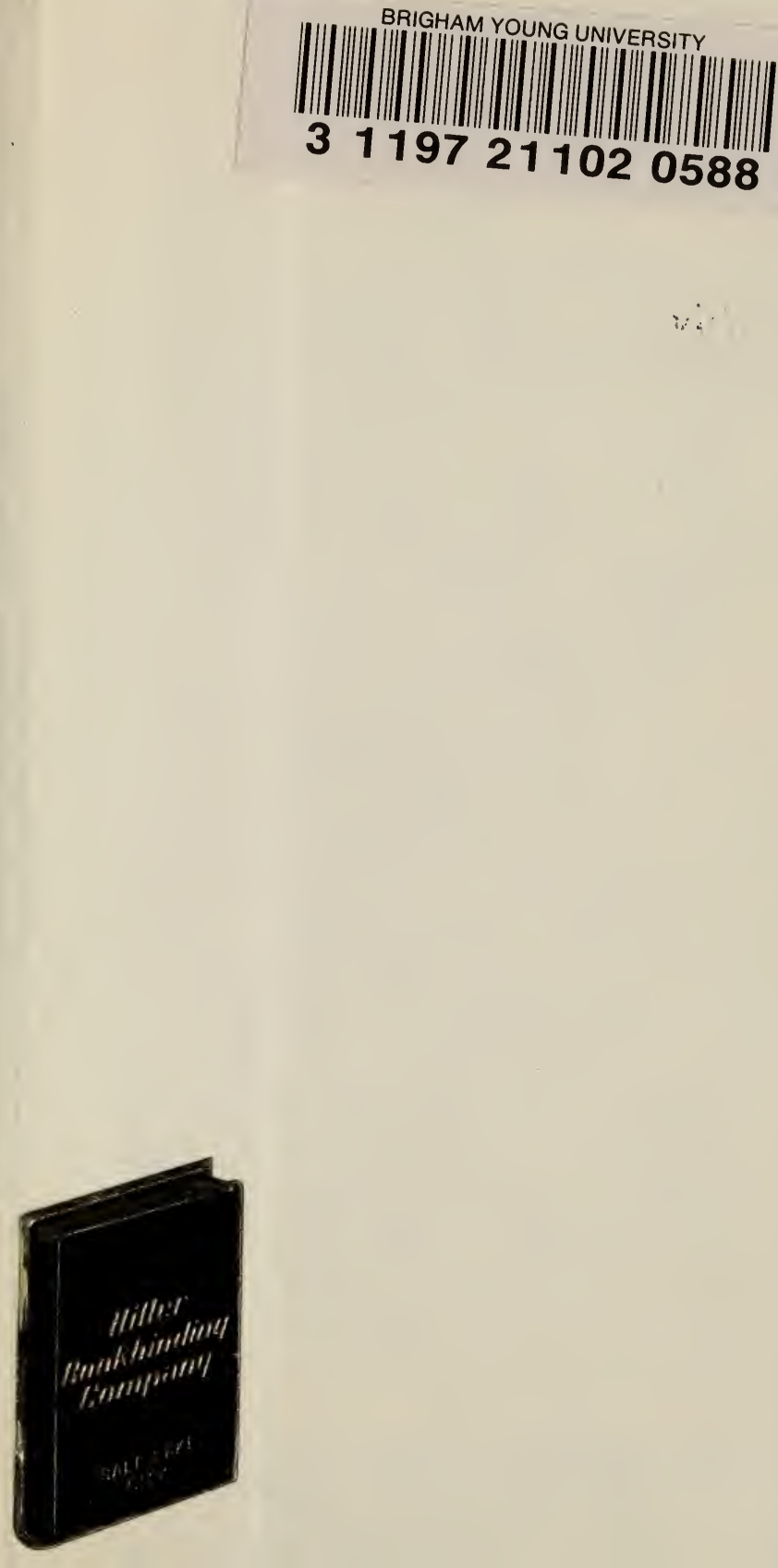
${ }^{3}, 80$

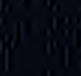

